\title{
AN EXPLORATION OF ACTIVE HARD MOUNT VIBRATION ISOLATION FOR PRECISION EQUIPMENT
}

Een verkenning van actieve trillingsisolatie voor precisiemachines met stijve ondersteuning

Gerrit Wijnand van der Poel 
This research was carried out under project number IPT04009A in the framework of the Innovation-oriented Research Programme (IOP) Precision Technology as implemented by AgentschapNL on behalf of the Dutch Ministry of Economic Affairs (http://www.agentschapNL.nl/iopprecisietechnologie).

Front and back cover: Carta del Cantino, 1502 AD.

World map from the Age of Exploration, showing Portuguese discoveries in the east and west.

The image has been reproduced with permission of the Italian Ministry of Cultural Heritage and Activities (Ministero per i Beni e le Attività Culturali).

An exploration of active hard mount vibration isolation for precision equipment

Poel, Gerrit Wijnand van der

ISBN 978-90-365-3016-3

(C) 2010 G. W. van der Poel, Enschede, The Netherlands.

Printed by Ipskamp Drukkers. 


\section{AN EXPLORATION OF \\ ACTIVE HARD MOUNT \\ VIBRATION ISOLATION \\ FOR PRECISION EQUIPMENT}

\section{PROEFSCHRIFT}

ter verkrijging van

de graad van doctor aan de Universiteit Twente,

op gezag van de rector magnificus,

prof. dr. H. Brinksma,

volgens besluit van het College voor Promoties

in het openbaar te verdedigen

op vrijdag 21 mei 2010 om 13:15 uur

door

Gerrit Wijnand van der Poel

geboren op 28 mei 1978

te 's-Gravendeel 
Dit proefschrift is goedgekeurd door: prof. ir. H.M.J.R. Soemers, promotor prof. dr. ir. J.B. Jonker, promotor dr. ir. J. van Dijk, assistent-promotor 
"If we knew what it was we were doing, it would not be called research, would it?"

(Attributed to) Albert Einstein 



\section{Summary}

Vibrations due to environmental disturbances can cause a loss of accuracy in high-precision manufacturing and inspection equipment. In many cases, floor vibrations are the dominant mechanical disturbance source. To reduce the vibration levels due to floor motion, the equipment is commonly mounted on vibration isolation systems with relatively small support stiffness (called soft mounts). However, the low support stiffness may introduce difficulties in the response to disturbances other than floor vibrations and with the levelling of the equipment.

The objective of the research described in this thesis is to develop an alternative vibration isolation concept, which makes use of relatively stiff supports (so-called hard mounts). The increased support stiffness of these mounts circumvents the previously mentioned difficulties of soft mounts. Additional objectives for this vibration isolation concept are: improvement of the damping of the suspension modes as well as relevant structural modes of the supported equipment; and achievement of floor vibration isolation performance as achieved by state-of-the-art soft mounts.

The required additional damping in the suspension modes and structural modes is achieved by feedback control. It is shown that absolute motion sensors (i.e. accelerometers or geophones) are preferred over force sensors and displacement sensors when additional damping is to be achieved in the suspension modes as well as several structural modes. Conditions are derived for which the feedback control system (using absolute motion sensors) is practically collocated, resulting in a robustly stable feedback system.

To reduce the transmission of floor vibrations further, feedforward compensation of measured floor vibrations is applied. The optimal feedforward compensation partly depends on system dynamics that are generally unknown. Therefore, adaptation algorithms are used to update the feedforward controller parameters in order to find the optimal feedforward compensation.

By using a Finite Impulse Response (FIR) parametrization, the Filtered-reference Least Mean Squares (FxLMS) algorithm, which is widely used in Active 
Noise Control applications, can be used to update the controller parameters. In this case, the instantaneous squared error has a global minimum. Moreover, the algorithm is fairly robust to modelling errors. Several existing extensions to the FxLMS algorithm are implemented in order to prevent actuator saturation, improve the convergence rate and shape the performance in the frequency domain. Unfortunately, the FIR parametrization requires a large number of parameters to model the optimal feedforward controller with sufficient accuracy. Therefore, the real-time computational capacity is found to be an important performance limitation, especially for multiple input, multiple output (MIMO) systems.

As an alternative, an Infinite Impulse Response (IIR) parametrization with fixed poles is proposed in this thesis. The same adaptation algorithms can be used for this parametrization and the adaptation problem is still convex in the controller weight parameters. It is shown that a similar performance can be achieved with less parameters, resulting in less demanding computational requirements. However, the performance depends critically on the choice of the fixed poles in the IIR filter.

In general, exact-constraint mechanical design of the supports is desired to prevent unpredictable deformations due to e.g. thermal loading. Moreover, deviating from the exact-constraint design has implications for the feedback and feedforward performance as well. Based on the analysis of a planar model, a guideline for the ratio of principal and parasitic stiffness of the supports has been derived: to reduce the influence of parasitic stiffness to an acceptable level, this stiffness ratio should at least be larger than 100 .

Requirements for the absolute motion sensors that are used in the hard mount vibration isolation system are derived from a generic sensor model and assumptions on the vibration input levels. It is shown that very stringent requirements are posed on these sensors, in terms of bandwidth and noise level (referred to input). No sensors has been found that meets the requirements over the entire frequency range. As a result, the sensor noise characteristics are an important limiting factor for the achievable vibration isolation performance.

The feedback and feedforward control strategies are verified by tests on a hard mount experimental setup which allows motion in only one direction. The experiments show that the feedback control achieves significant damping in the suspension mode $(\zeta>70 \%)$ and structural mode $(\zeta>30 \%)$ of the setup. The increased damping reduces the root mean square (RMS) acceleration levels by $15-20 \mathrm{~dB}$ compared to the open loop response. At a floor acceleration of approx. $20 \mathrm{~mm} / \mathrm{s}^{2}$ RMS $(0-1600 \mathrm{~Hz})$, the feedforward compensation (in combination with the feedback control) is able to reduce the residual acceleration level to $0.8-1.7 \mathrm{~mm} / \mathrm{s}^{2}$ RMS $(-27$ to $-21 \mathrm{~dB})$. 
The floor vibration isolation performance that is achieved experimentally is not yet comparable to the performance of a state-of-the-art soft mount. However, the obtained results are encouraging, especially when taking into account the much larger support stiffness of the experimental setup (by at least a factor 300) compared to that of a soft mount system. Currently, the performance is primarily limited by the noise level in the motion sensors and delay due to the anti-aliasing filters.

Further research is required to improve the vibration isolation performance and develop the vibration isolation system. This research should focus on implementation on a MIMO setup, more efficient implementation of the adaptive algorithms and improvement of the sensors. 



\section{Contents}

Summary

$\begin{array}{lll}\text { Contents } & \mathrm{v}\end{array}$

Nomenclature $\quad$ xi

1 Introduction 1

1.1 Application background ................ 1

1.2 Research objectives ................. 3

1.3 Thesis outline ...................... 3

2 Vibration isolation: principles and state of the art 5

2.1 Introduction ................... 5

2.2 Characterization of disturbances . . . . . . . . . . 7

2.2.1 Direct disturbances .............. 7

2.2.2 Indirect disturbances . . . . . . . . . . . 8

2.3 Evaluating disturbance effects . . . . . . . . . . . . 10

2.4 Basic system model . . . . . . . . . . . . . . . . 11

2.4 .1 Dynamic modelling . . . . . . . . . . . 13

2.4 .2 Parameter values . . . . . . . . . . . . 14

2.5 Passive vibration isolation . . . . . . . . . . . . 14

2.6 Active vibration isolation . . . . . . . . . . . 17

2.6.1 Active vibration isolation using feedback control . . . 17

2.6.2 Active vibration isolation using feedforward control . . 23

2.7 State of the art . . . . . . . . . . . . . 24 
3 Active hard mount concept $\quad 27$

3.1 Performance objectives . . . . . . . . . . . . 27

3.2 Conceptual system design . . . . . . . . . . . . . 31

3.2 .1 Control strategy . . . . . . . . . . . . 31

3.2.2 Mechanical design concept . . . . . . . . . . 32

3.2.3 Effects of non-ideal constraints . . . . . . . . 36

3.3 Recapitulation ................... 41

4 Active damping by feedback control 43

4.1 System model . . . . . . . . . . . . . . . 44

4.2 Limitations of a 1 DOF controller . . . . . . . . . . 45

4.2.1 2 DOF control configurations .......... 47

4.3 DVF(-like) control . . . . . . . . . . . . . 48

4.3.1 Stability robustness of DVF-like control . . . . . . . 51

4.4 Recapitulation ...................... 56

5 Feedforward compensation of floor vibrations 57

5.1 System model . . . . . . . . . . . . . . 57

5.1.1 Internal model compensation . . . . . . . . . 59

5.2 Optimal feedforward control . . . . . . . . . . . . . 60

5.2.1 Performance measure . . . . . . . . . . 60

5.2.2 Causal Wiener filter . . . . . . . . . . . 62

5.2.3 Effects of measurement noise ........... 65

5.2 .4 Control effort weighing . . . . . . . . . . 68

5.2.5 Conclusions and final remarks . . . . . . . . . 69

5.3 Adaptive feedforward control ... . . . . . . . . 70

5.3.1 Filtered-X LMS algorithm . . . . . . . . . . 71

5.3.2 Improving the convergence rate . . . . . . . . 75

5.3.3 Effects of errors in the secondary path model . . . . . 77

5.3.4 Preventing actuator signal clipping . . . . . . . . . 79

5.3.5 Filter parametrizations ............ 81

5.3.6 Computational requirements .......... 85

5.4 Recapitulation ....................... 86 
6 Sensors and actuators for vibration isolation systems

6.1 Absolute motion sensors . . . . . . . . . . . . . . 89

6.1.1 Generic model of absolute motion sensors . . . . . . . 90

6.1 .2 Sensor requirements .............. . 93

6.1.3 Determining the noise level of seismic sensors . . . . 97

6.1.4 Noise level of the Endevco 7703A-1000 . . . . . . . 98

6.1.5 Noise level of the GeoSpace GS-11D . . . . . . . . . 103

6.1.6 Comparison of evaluated sensors . . . . . . . . 107

6.1.7 Recapitulation and final remarks . . . . . . . . . 109

6.2 Actuators ....................... 110

6.2 .1 Actuator requirements .............. 110

6.2.2 Piezoelectric stack actuator . . . . . . . . . 113

6.2 .3 Voice coil actuator . . . . . . . . . . . . . 119

6.2.4 Recapitulation and final remarks . . . . . . . . . 123

7 Experimental setup $\quad 125$

7.1 Description of the setup ................ 125

7.2 Parametric modelling . . . . . . . . . . . . . 128

7.2.1 Single-directional model . . . . . . . . . 129

7.2.2 Coupled 3D dynamics . . . . . . . . . . . . . 133

7.2.3 Signal conditioning and power electronics . . . . . . . 135

7.2.4 Combined electromechanical model . . . . . . . . 136

7.3 System identification . . . . . . . . . . . . . . 138

7.3.1 PEA-based setup . . . . . . . . . . . 140

7.3 .2 VCA-based setup ................. 146

7.4 Recapitulation .................... 149

8 Control experiments $\quad 151$

8.1 Performance criteria . . . . . . . . . . . . 151

8.2 PEA-based setup . . . . . . . . . . . . . . 153

8.2.1 Excitation level and target performance . . . . . . . 153

8.2.2 Feedback control ... . . . . . . . . . . 154

8.2 .3 Causal Wiener filter . . . . . . . . . . . . . 158 
8.2.4 Adaptive feedforward using FIR filters . . . . . . . . . . 161

8.3 VCA-based setup . . . . . . . . . . . . . 167

8.3.1 Excitation level and target performance . . . . . . 167

8.3.2 Feedback control ............... 168

8.3.3 Causal Wiener filter . . . . . . . . . . . . 171

8.3.4 Adaptive feedforward using FIR filters . . . . . . . . . 173

8.3.5 Adaptive feedforward using IIR filters . . . . . . . . . . 179

8.4 Recapitulation .................... 186

9 Conclusions and recommendations $\quad 189$

9.1 Conclusions ........................ 189

9.1.1 Experimental results .............. 190

9.1.2 Feedback control ................ 192

9.1.3 Feedforward control . . . . . . . . . . . 192

9.1 .4 Sensors . . . . . . . . . . . . . . . 193

9.1 .5 Actuators ................... 194

9.1.6 Mechanical design ............... 195

9.2 Recommendations for further research . . . . . . . . . . . 196

\section{Appendices}

A System models

A.1 Basic system model . . . . . . . . . . . . . . . . 199

A.2 Experimental setup models . . . . . . . . . . . . . 209

B Feedback controller tuning for active damping 215

B.1 Model equation ..................... 215

B.2 Maximum damping . . . . . . . . . . . . . 216

B.3 Passivity-based control . . . . . . . . . . . 217

C Causal Wiener filter $\quad 221$

C.1 (Co-) inner-outer factorization . . . . . . . . . . . 221

C.2 Derivation of the Causal Wiener filter . . . . . . . . . . . 223

C.3 Implement. of inner-outer factorization and causality operator 225 
D Geophone noise analysis

D.1 Geophone model and signal conditioning . . . . . . . . . 229

D.2 Noise analysis . . . . . . . . . . . . . 231

E Frequency domain subspace identification 237

E.1 System identification procedure . . . . . . . . . . 237

E.2 Model structure selection . . . . . . . . . . . . 238

E.3 Experiment design . . . . . . . . . . . . . . . 238

E.4 Data acquisition and preprocessing . . . . . . . . . . 240

E.5 Subspace identification . . . . . . . . . . . . . 242

E.6 Model selection and validation . . . . . . . . . . . . . . 249

E.7 Subspaces in linear algebra . . . . . . . . . . . . . . 250

F Notes on controller implementation $\quad 251$

F.1 Controller block diagrams . . . . . . . . . . . . 251

F.2 IIR filter implementation . . . . . . . . . . . . . 253

F.3 Transformation to block diagonal form . . . . . . . . . . 256

$\begin{array}{lr}\text { Samenvatting } & 259\end{array}$

$\begin{array}{ll}\text { Dankwoord } & 263\end{array}$

$\begin{array}{ll}\text { Publications } & 265\end{array}$

$\begin{array}{lr}\text { Bibliography } & 267\end{array}$ 



\section{Nomenclature}

\section{Notational conventions and operators}

\begin{tabular}{|c|c|}
\hline$x$ & Scalar variable/signal (italic, lower case) \\
\hline$x$ & Vector variable/signal (bold italic, lower case) \\
\hline$X$ & $\begin{array}{l}\text { Scalar constant, Fourier/Laplace/ } z \text {-transform (scalar), or } \\
\text { SISO transfer function (italic, upper case) }\end{array}$ \\
\hline$X$ & $\begin{array}{l}\text { Matrix constant, Fourier/Laplace/ } z \text {-transform (vector), or } \\
\text { MIMO transfer function (bold italic, upper case) }\end{array}$ \\
\hline$\dot{\bullet}$ & First derivative of $\bullet$ with respect to time \\
\hline$\ddot{\theta}$ & Second derivative of $\bullet$ with respect to time \\
\hline$\hat{\bullet}$ & Model of the dynamic system $\bullet$, or estimate of the signal $\bullet$ \\
\hline$\bullet(t)$ & Continuous time signal \\
\hline$\bullet(k)$ & Sampled signal at time instance $k$ \\
\hline$\bullet(s)$ & Laplace transformed variable or continuous system \\
\hline$\bullet(z)$ & $z$-transformed variable or discrete system \\
\hline$\bullet(\omega)$ & Fourier transformed variable, or value of $\bullet$ at $\omega$ \\
\hline$\bullet(j \omega)$ & Value of $\bullet$ at $s=j \omega$ \\
\hline$\bullet\left(\mathrm{e}^{j \omega}\right)$ & Value of $\bullet$ at $z=\mathrm{e}^{j \omega}(-\pi \leq \omega \leq \pi)$ \\
\hline$\bullet(f)$ & Value of $\bullet$ at $s=j 2 \pi f$ or $z=\mathrm{e}^{j 2 \pi f T_{s}}$ \\
\hline$\bullet_{c i}(z)$ & Co-inner factor of the discrete system $\bullet$ \\
\hline$\bullet_{c o}(z)$ & Co-outer factor of the discrete system $\bullet$ \\
\hline$\bullet_{i}(z)$ & Inner factor of the discrete system $\bullet$ \\
\hline$\bullet_{o}(z)$ & Outer factor of the discrete system $\bullet$ \\
\hline$|\bullet|$ & Absolute value or magnitude of $\bullet$ \\
\hline$\angle \bullet$ & Angle of the complex number $\bullet\left[{ }^{\circ}\right]$ \\
\hline$\|\bullet\|$ & Norm of $\bullet$ \\
\hline$\|\bullet\|_{F}$ & $\begin{array}{l}\text { Frobenius norm of } \bullet \\
\text { (square root of sum of squared elements of } \bullet \text { ) }\end{array}$ \\
\hline$\|\bullet\|_{p}$ & $p$-norm of the vector $\bullet(p=0,1,2, \ldots, \infty)$ \\
\hline
\end{tabular}




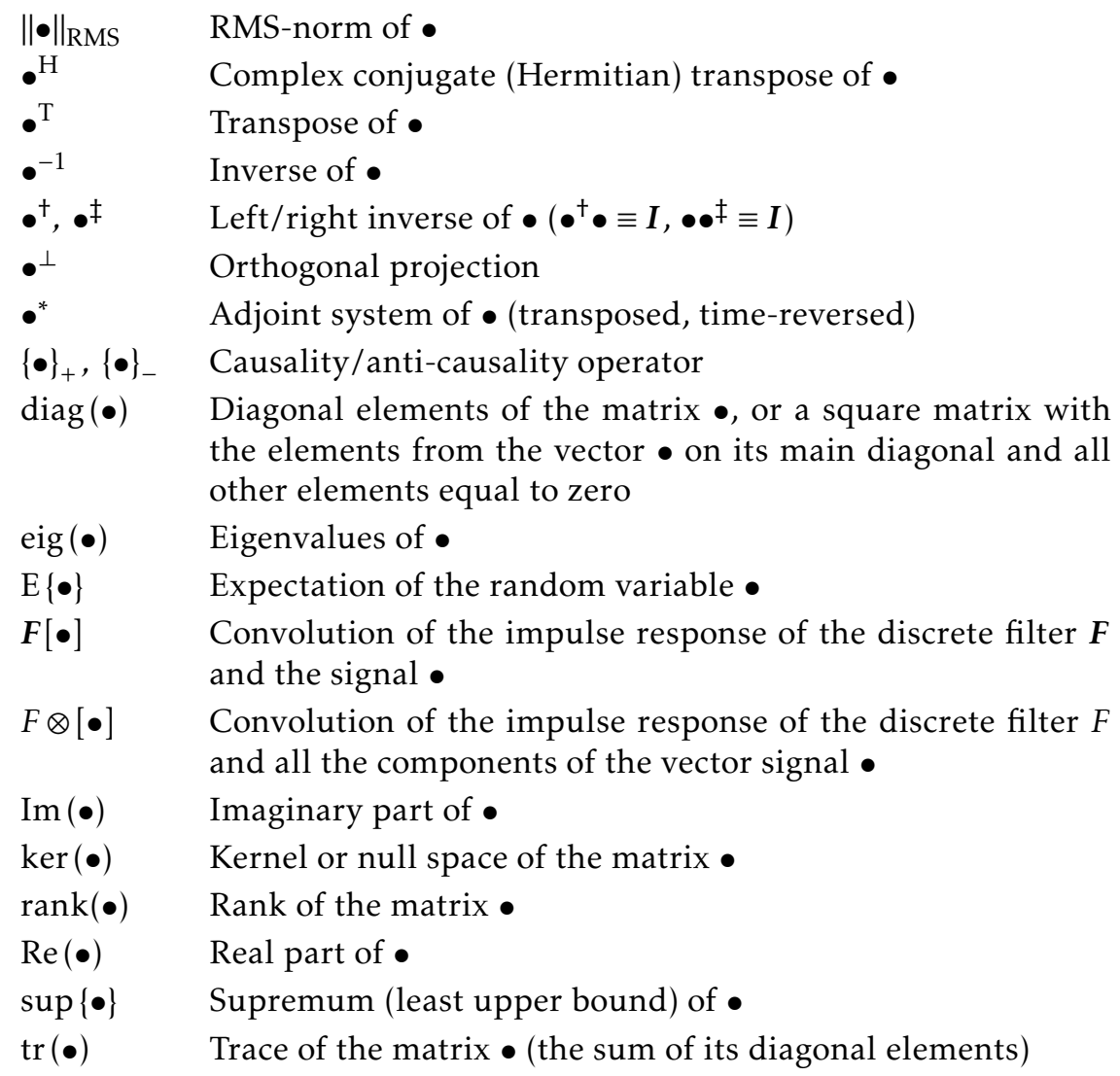

\section{Abbreviations}
$\mathrm{AD}, \mathrm{ADC}$
Analog-to-digital (converter)
ANC
Active noise control
CSD
Cross-power spectral density
DA, DAC
Digitial-to-analog (converter)
DOF
Degree of freedom
DVF
Direct velocity feedback
FB
Feedback
FF
Feedforward
FFT
Fast Fourier transform
FIR
Finite impulse response
FOS
First order section
FRF
Frequency response function 


$\begin{array}{ll}\text { FxLMS } & \text { Filtered reference LMS } \\ \text { FxNLMS } & \text { Filtered reference, normalized LMS } \\ \text { HP } & \text { High-pass } \\ \text { IFF } & \text { Integral force feedback } \\ \text { IIR } & \text { Infinite impulse response } \\ \text { IMC } & \text { Internal model compensation } \\ \text { ISE } & \text { Instantaneous squared error } \\ \text { LMS } & \text { Least mean square } \\ \text { LP } & \text { Low-pass } \\ \text { LTI } & \text { Linear, time-invariant } \\ \text { MEMS } & \text { Micro-electromechanical system(s) } \\ \text { MIMO } & \text { Multiple input, multiple output } \\ \text { MSE } & \text { Mean square error } \\ \text { NHNM } & \text { New High Noise Model } \\ \text { NLMS } & \text { Normalized LMS } \\ \text { NLNM } & \text { New Low Noise Model } \\ \text { NMP } & \text { Non-minimum phase } \\ \text { OL } & \text { Open loop } \\ \text { PD } & \text { Proportional-derivative } \\ \text { PE, PEA } & \text { Piezoelectric (actuator) } \\ \text { PI } & \text { Proportional-integral } \\ \text { pk-pk } & \text { Peak-to-peak value of a signal } \\ \text { PSD } & \text { Power spectral density } \\ \text { RMS } & \text { Root mean square } \\ \text { RTI, RTO } & \text { Referred to input or output } \\ \text { SISO } & \text { Single input, single output } \\ \text { SNR } & \text { Signal-to-noise ratio } \\ \text { SOS } & \text { Second order section } \\ \text { SPR } & \text { Strictly positive-real } \\ \text { SVD } & \text { Singular value decomposition } \\ \text { VCA } & \text { Voice coil actuator } \\ \text { VC } & \text { Vibration Criterion } \\ & \\ \text { INA } & \end{array}$

\section{Latin(-like) symbols}

$\mathbb{C} \quad$ Set of complex numbers

$\mathbb{N} \quad$ Set of natural numbers (positive integers, excluding 0 )

$\mathbb{R} \quad$ Set of real numbers 
$\boldsymbol{d}(k) \quad$ Disturbance signals for (adaptive) feedforward controller

$\boldsymbol{d}_{e}(k) \quad$ Error signal component due to the disturbance signals

$\boldsymbol{e}(k) \quad$ Error signals for (adaptive) feedforward controller

$f \quad$ Physical frequency $[\mathrm{Hz}]$

$f_{s} \quad$ Sample frequency $[\mathrm{Hz}]$

$g \quad$ Earth's gravity $\left(g=9.81\left[\mathrm{~m} / \mathrm{s}^{2}\right]\right)$

$j=\sqrt{-1} \quad$ Imaginary unit

$k_{B} \quad$ Boltzmann's constant $\left(k_{B}=1.38 \cdot 10^{-23}[\mathrm{~J} / \mathrm{K}]\right)$

$m_{i} \quad$ Floor mass $(i=0) /$ Machine mass $1,2(i=1,2)[\mathrm{kg}]$

$\boldsymbol{n}_{e}(k) \quad$ Measurement noise on error signals

$\boldsymbol{n}_{r}(k) \quad$ Measurement noise on reference signals

$p(s), p_{\mathrm{r}}(s) \quad$ Characteristic polynomial

$r(k) \quad$ Reference signals for (adaptive) feedforward controller

$u, \boldsymbol{u} \quad$ Input (control) signal(s)

$w(k) \quad$ Vector of adaptive feedforward controller weights

$\Delta x \quad$ Machine deformation $\left(x_{2}-x_{1}\right)[\mathrm{m}]$

$x(k) \quad$ Reference signal component due to the disturbance signals

$x_{i} \quad$ Displacement of mass $i(i=0,1,2)[\mathrm{m}]$

$y, y \quad$ Output (measurement) signal(s)

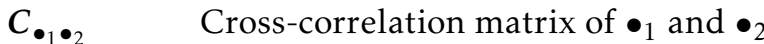

$C_{i}(s) \quad$ Compliance transfer function $(i=0,1,2)[\mathrm{m} / \mathrm{N}]$

$D_{i}(s) \quad$ Deformation compliance transfer function $(i=0,1,2)[\mathrm{m} / \mathrm{N}]$

$\boldsymbol{F}(z) \quad$ Regression filter

$F_{a} \quad$ Control actuator force [N]

$F_{d}, F_{d_{i}} \quad$ Disturbance force (acting on mass $\left.i\right)[\mathrm{N}]$

$F_{\text {sh }} \quad$ Shaker force $[\mathrm{N}]$

$\boldsymbol{I}, \boldsymbol{I}_{N} \quad(N \times N)$ identity matrix

ISE Instantaneous squared error cost criterion

$J_{\text {MSE }} \quad$ Mean squared error cost criterion

$K, K_{1}, K_{2}(s) \quad$ (SISO) feedback controller transfer function

$L \quad$ Number of adaptive feedforward controller weights

$L(s) \quad$ Loop gain transfer function

$M(z) \quad$ Complementary noise shaping filter

$M_{i} \quad$ Major bodies in experimental setup ( $i=0$ : floor, $i=1$, 2: machine model)

$N(z) \quad$ Noise shaping filter

$N . \quad$ Number of elements in the vector $\bullet$

$P$.• $\quad($ Cross) power spectral density matrix of the vector signal $\bullet$ 
$P(z), \boldsymbol{P}(z) \quad$ Primary path, transfer function from disturbance signal(s) to error signal(s)

$P_{2}(z) \quad$ Primary path 2 , transfer function from disturbance signal to validation signal

$Q(z), Q(z) \quad$ Quaternary path, transfer function from control signal(s) to reference signal(s)

$\boldsymbol{R}_{\bullet} \quad$ Autocorrelation matrix of $\bullet$

$S(z), S(z) \quad$ Secondary path, transfer function from control signal(s) to error signal(s)

$S_{2}(z) \quad$ Secondary path 2, transfer function from control signal to validation signal

$T(z), T(z) \quad$ Tertiary path, transfer function from disturbance signal(s) to reference signal(s)

$T \quad$ Absolute temperature $[\mathrm{K}]$

$T$ (s) Transmissibility transfer function [-]

$T_{\text {ref }}(s) \quad$ Reference transmissibility transfer function [-]

$T_{d}(s) \quad$ Deformation transmissibility transfer function $\left[s^{2}\right]$

$\boldsymbol{W}(z) \quad$ Feedforward controller

$W(k) \quad$ Adaptive feedforward controller

\section{Greek symbols}

$\beta \quad$ Leakage factor for the weight update

$\zeta \quad$ Damping ratio [-] or [\%]

$\mu(k) \quad$ User-defined, (possibly) time-varying update step size

$\bar{\mu} \quad$ User-defined normalized update step size

$\bar{\sigma} \quad$ Largest singular value

$\psi(k) \quad$ Regression vector

$\psi_{S}(k) \quad$ Filtered regression vector

$\omega$

Angular frequency [ $\mathrm{rad} / \mathrm{s}]$, or normalized angular frequency

$(-\pi \leq \omega \leq \pi)$

$\omega_{a} \quad$ Anti-resonance frequency $[\mathrm{rad} / \mathrm{s}]$

$\omega_{r} \quad$ Structural resonance frequency $[\mathrm{rad} / \mathrm{s}]$

$\omega_{\text {susp }} \quad$ Suspension resonance frequency $[\mathrm{rad} / \mathrm{s}$ ]

Ф. Spectral factor of the random (vector) signal 



\section{Chapter 1}

\section{Introduction}

In section 1.1, several applications are presented that require precision equipment. To achieve the required accuracy in these precision machines, disturbances must be adequately dealt with. Vibration isolation systems specifically aim at reducing the vibration levels in a machine. In this thesis, a novel vibration isolation concept is investigated. The research objectives are discussed in section 1.2. Finally, the outline of the thesis is discussed in section 1.3.

\subsection{Application background}

Over the last decades, the technology development in many fields of engineering and research has focussed on miniaturization and increasing accuracy. Perhaps the best known example is found in the semiconductor industry. For over four decades, "Moore's Law" has been a driving force behind the development of integrated circuit (IC) technology. It is based on Gordon Moore's observation of an exponential decrease in IC component size [Moore (1965)]. This decrease in component size has led to the widespread availability of powerful computer chips as well as large-capacity data storage and memory modules.

Moreover, new fields of research have emerged, such as micro electromechanical systems (MEMS) and microfluidics (e.g. the so-called lab-on-a-chip), which build on the production technology developed by the semiconductor industry.

Another field of research that is concerned with increasingly smaller scales is the field of materials science and engineering. Various scientific instruments such as (scanning or transmission) electron microscopes or scanning probe microscopes (e.g. atomic force microscopes) are commonly used to inspect samples for e.g. surface roughness, constitution or crystalline structure. 
Finally, the development of new (linear) particle accelerators for physics experiments on subatomic particles is mentioned as an area where increasing accuracy is desired, see e.g. the project websites [EuroTeV; SLAC].

A common denominator in the mentioned industries and fields of research, is their need for high-precision equipment. The required accuracies range from a few Ångströms to several tens of nanometres. These (ever increasing) accuracy demands, in some cases combined with throughput requirements, pose challenging design objectives for the manufacturers of high-precision equipment. Besides obtaining the desired functionality, the design also has to take into account any environmental disturbances that can limit the overall accuracy. These disturbances can include mechanical loads (vibrations), thermal loads, electromagnetic radiation as well as contaminations and humidity.

The design of such equipment is commonly known as precision engineering and has to take into account a wide range of topics, e.g. physics, optics, kinematics, dynamics, materials selection, thermal management and control. A more in-depth discussion of some of these topics can be found in e.g. [Slocum (1992); Nakazawa (1994); Koster (1998); Schellekens et al. (1998); Blanding (1999)].

\section{Dealing with disturbances}

In general, dealing adequately with disturbances requires a robust machine design as well as disturbance rejection measures. Robust machine design aims at reducing the intrinsic sensitivity of the machine to disturbances, for example:

- stiff and lightweight design, which results in as little deformation of the structure as possible under dynamic loads

- exact-constraint design, which prevents unpredictable deformations due to thermal stresses

Disturbance rejection can be achieved by controlling the machine's operating environment. On a (very) large scale, this can be achieved by minimizing the effect of disturbances throughout the complete facility design. This may include site selection, facility layout, floor design and clean room facilities.

However, typically, machines are equipped with their own disturbance rejection systems. Some commonly used systems include:

- soundproof enclosures

- cooling systems

- servo-controlled positioning systems

- vibration isolation systems 


\subsection{Research objectives}

Typical industrial vibration isolation systems focus on reducing the effects of floor vibrations. To this end, these systems have a relatively low support stiffness (so-called soft mounts). However, very low support stiffness values lead to problems with the levelling of the supported machine and increase the susceptibility to direct disturbances, i.e. disturbances that act directly on the machine.

These problems can be circumvented by increasing the support stiffness. However, without any additional measures, the higher stiffness would significantly increase the transmission of floor vibrations to the machine. Therefore, an active control system is required to improve the response to floor vibrations.

The aim of this research project is therefore

to develop a vibration isolation system that combines high support stiffness with adequate isolation of floor vibrations.

The development of the active control system is the main subject of this thesis. The control system uses feedback control to improve the damping in the system, in combination with feedforward compensation of measured floor vibrations. For the feedforward control, control concepts are considered that are carried over from the field of Active Noise Control (ANC), which is closely related to vibration isolation.

Moreover, much attention is paid to the requirements for the mechanical design as well as the sensors and actuators. This is necessary as the mechanical design and the choice of sensors and actuators have a significant influence on the overall vibration isolation performance that can be achieved by the active control system.

\subsection{Thesis outline}

In chapter 2, the principles of passive and active vibration isolation are explained. Moreover, the characteristics of disturbances are discussed. The chapter concludes with a discussion of the current state of the art and some recent developments in the field of vibration isolation.

In chapter 3, the performance objectives for the hard mount vibration isolation system are presented. Moreover, the mechanical design concept is presented and the effect of non-ideal mechanical behaviour on the control performance is analysed. Based on the results of this theoretical analysis, guidelines for the mechanical design are formulated. 
In chapter 4, design methods for the feedback controller are discussed, which aim at improving the suspension mode damping and the (relevant) structural mode damping. The suitability of several sensor types for adding structural damping are analysed.

In chapter 5, the feedforward compensation of floor vibrations is discussed in detail. The optimal causal controller is presented. From these optimization results, several factors are identified that can limit the achievable vibration isolation performance. It turns out that the optimal controller can not be computed in practice. Therefore, an adaptive feedforward control scheme is used to find the optimal controller. This scheme is based on the Filtered-x LMS algorithm that is widely used in ANC applications. Several extensions to the algorithm are presented that improve the stability or the convergence rate of the adaptation.

In chapter 6, several absolute motion sensors and two actuators are tested for their applicability in active hard mount vibration isolation systems. It turns out that the noise characteristics of sensors and actuators are dominant factors in the overall vibration isolation performance

In chapter 7, the modelling of the experimental setup that is used for controller experiments is discussed. Two versions of the setup are described, which have different actuator types and different support stiffness. Moreover, the results from system identification experiments are presented.

In chapter 8 , the results of the controller experiments are presented that have been performed on the two experimental setups. The feedback control methods of chapter 4 and the adaptive feedforward control methods of chapter 5 have been tested on both setups.

In chapter 9, the conclusions from the various theoretical and experimental results that have been presented in this thesis are summarized. Moreover, recommendations for further research are discussed. 


\section{Chapter 2}

\section{Vibration isolation: principles and state of the art}

In this chapter the principles of vibration isolation are discussed. Firstly, a bird's eye view of the design of a vibration isolation system is presented. Consequently, the characteristics of disturbances and an evaluation method for isolation performance are discussed. Moreover, the principles of passive and active vibration isolation are explained. The chapter concludes with a discussion of the current state of the art and some recent developments in the field of vibration isolation.

\subsection{Introduction}

For precision equipment, the process or product quality is highly dependent on the alignment of several critical machine parts during operation. Disturbance sources can cause vibrations in the equipment, resulting in a (temporary) loss of accuracy. Commonly, vibration isolation is applied to reduce the vibration levels to acceptable levels, thereby assuring the correct operation of the machine. As illustrated in figure 2.1, three important factors can be recognized in any vibration isolation problem:

1. the sensitive equipment; referred to as the machine ${ }^{1}$

2. one or more disturbance sources;

3. the vibration isolation system; comprised of the machine mounts and (possibly) sensors, actuators and their accessories

1 Note that the sensitive equipment may actually be only a component of a larger machine. 


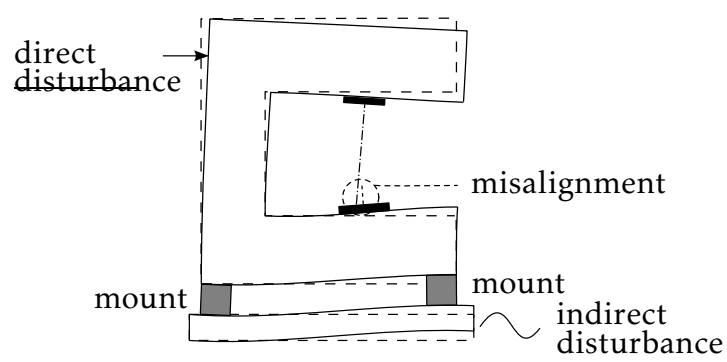

Figure 2.1: Illustration of deformations in a machine due to various disturbances, leading to a reduction in the machine's accuracy. The machine mounts may be equipped with sensors and actuators in order to reduce the effects due to these disturbances.

A vibration isolation system is said to provide passive isolation when the isolation performance is solely based on the mechanical design of the machine supports. In active vibration isolation systems the machine supports incorporate actuators, sensors and a control system. These active components allow improvement of the vibration isolation performance compared to the passive behaviour. The basic principles of passive and active vibration isolation and their current state-of-the-art are discussed in sections 2.5, 2.6 and 2.7, respectively.

Before examining these vibration isolation principles more closely, it is useful to briefly discuss the requirements that have to be fulfilled by a vibration isolation system. This is by no means a simple task, and is usually very application-dependent. A generic, implicit definition of the design requirements can be stated as:

The design requirements for any vibration isolation system follow from the discrepancy between the desired accuracy level and the expected accuracy level, which would be obtained without any vibration isolation system.

The first difficulty that arises is the definition of the (desired) accuracy level. This definition must include a clear indication which critical components are involved. Moreover, a specific, measurable performance criterion must be defined. Several performance criteria and a method to evaluate these criteria are discussed in section 2.3 .

Once the desired accuracy level is defined, the next step is to predict the achievable accuracy from a dynamic model of the machine, which is subjected to various disturbances. Hence, a vital step is to determine the disturbance levels which may be expected during operation. The characteristics of various disturbances are discussed in more detail in section 2.2. 


\subsection{Characterization of disturbances}

There are many ways to distinguish between different disturbance types. One possibility is to distinguish between deterministic disturbances and random disturbances. The latter may also be referred to as broadband disturbances. Periodical and, more specifically, (multi-)tonal disturbances are important subsets of deterministic disturbance signals.

Another distinction can be made based on the stationarity of the disturbances. For periodical disturbances, this means that the signal spectrum is invariant over time. For random disturbances, strict stationarity requires that the probability density function is time-invariant. In many cases the less strict wide sense stationarity is sufficient, which implies that only the mean and variance of the random process are time-invariant.

Although the distinctions mentioned above are used on occasion in this thesis, the main distinction in this thesis is based on the way disturbances enter the machine. The disturbances are dubbed either direct or indirect disturbances, see also figure 2.1. Direct disturbance sources are forces which act directly on the machine, causing it to vibrate. Indirect disturbances are vibrations which originate from the support structure and are transmitted through the machine supports. The latter disturbances will be informally referred to as floor vibrations, although it should be recognized that the floor vibrations are actually the result of the indirect disturbances and not a disturbance source by itself. However, it is often convenient to consider floor vibrations instead of the actual indirect disturbance sources.

In the following subsections, some direct and indirect disturbance sources and their characteristics are discussed. Moreover, the approach to evaluating the effects of multiple disturbance sources is discussed.

\subsubsection{Direct disturbances}

Direct disturbance sources vary greatly between different applications, but may include:

- Reaction forces on the machine due to stage motion

- Forces transmitted through power or data cables

- Forces generated by vacuum pumps (due to unbalance)

- Forces transmitted by the cooling water system [Redaelli et al. (2002)]

- Acoustic excitation [Gendreau (1999); Roozen et al. (2006); Roozen and Vervoordeldonk (2007)] 
It is expected that most of these sources are random in nature, except for the reaction forces due to stage motion and the forces generated by vacuum pumps, which are mostly deterministic.

Moreover, for active vibration isolation systems the active components (sensors and actuators and their amplifiers) should also be considered as disturbance sources. The injected noise will cause the actuator to generate random forces, thus adding to some extent to the vibration level.

\subsubsection{Indirect disturbances}

The indirect disturbances which are observed at the machine mounts are dominantly caused by ground vibrations and disturbance sources inside the building. Additionally, building sway due to wind loading can result in large horizontal motion at elevated floor levels.

\section{- Disturbance sources inside buildings:}

Inside a building, disturbances originate from e.g. workshop machinery, climate control systems and human activity (footfall, closing doors). In the case of poorly balanced rotating machinery, tonal disturbances may be significant, otherwise random disturbances will dominate. The disturbances related to human activity are usually of a repetitive-impact nature.

\section{- Ground vibrations:}

Ground vibrations have been studied to a great extent by seismologists and the designers of particle accelerators, see e.g. [Peterson (1993); Sery and Napoly (1996); Bialowons et al. (2007)]. These vibrations are generally random in nature. Commonly, a distinction is made between "slow" ( $f \leq 1 \mathrm{~Hz})$ and "fast" vibrations $(f>1 \mathrm{~Hz})$. Some of the sources that have been identified to cause the "slow" vibrations are (remote) earthquakes, atmospheric pressure changes, ocean swell [Friedrich et al. (1998)] and temperature variations. The actual motion level at a site depends on the soil type, and may show seasonal variation as well. The New High Noise Model (NHNM) and the New Low Noise Model (NLNM), which are based on numerous measurements from seismological stations around the world [Peterson (1993)], may be used as a guideline for "slow" ground vibrations.

On the other hand, "fast" vibrations are dominated by so-called cultural noise, which is caused by human activities such as road traffic and rail traffic. Obviously, the cultural noise level is highly site-dependent. It may also vary from day to night and from weekdays to weekends. 


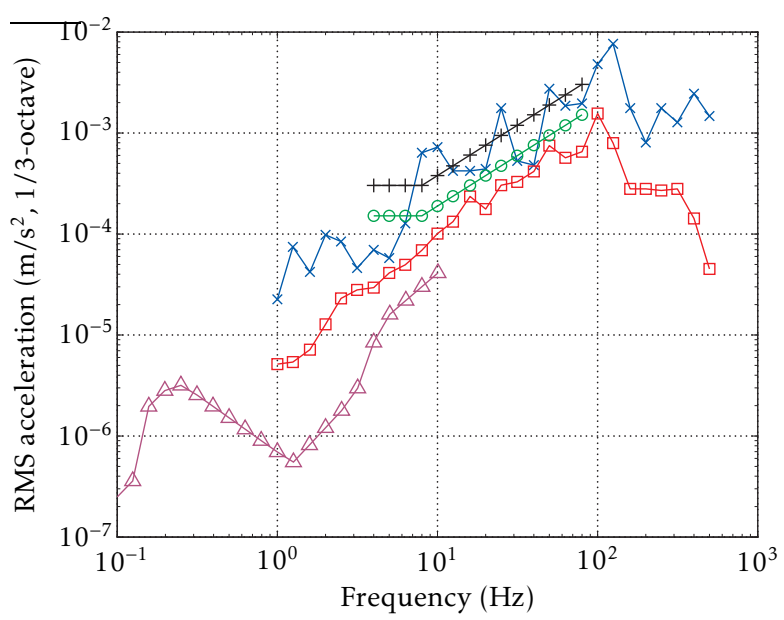

Figure 2.2: Comparison of floor vibration measurements (courtesy of FEI Company [Visscher (2006)]) with VC-curves D \& E and the NHNM model $*:$ measurements (maximum); $\quad+:$ VC-D $(6 \mu \mathrm{m} / \mathrm{s}) ; \quad \triangle:$ NHNM $\square:$ measurements (median); $\bigcirc$ : VC-E $(3 \mu \mathrm{m} / \mathrm{s})$;

Determining the expected floor vibration levels at a certain site is a difficult task, as the vibration levels usually vary over time. Although (sufficiently long) measurements at the specific site offer the best information, it may be impractical to measure each separate site. If available, measurements of previous, similar sites could be used. Alternatively, a dynamic model of the site and some suitable input signals could be used. Again, previous data may be used as a reference. Models of floor dynamics have been reported by e.g. [Petyt and Mirza (1972); Amick et al. (1991); Howard and Hansen (2003)].

In the early stages of the design, the Vibration Criterion (VC) curves may be used as a rule of thumb [Gordon (1991)]. These curves are based on the observation that many floor vibration spectra can be roughly approximated by a flat spectrum when expressed as root mean square (RMS) velocity on a one-third octave frequency grid from approximately 4 to $80 \mathrm{~Hz}$, with a slight increase below $8 \mathrm{~Hz}$. As the name implies, this logarithmic frequency grid has three bins per octave.

In figure 2.2, the VC-D and VC-E curves (respectively 6 and $3 \mu \mathrm{m} / \mathrm{s}$ RMS in one-third octave bandwidth) are compared with actual floor acceleration measurements (courtesy of FEI Company [Visscher (2006)]). For this purpose, the VC-curves have been converted to acceleration values. The (vertical) acceleration measurements were obtained from six different sites. For proprietary reasons, only the maximum and median of the measured sites are plotted at each frequency bin. For comparison, the RMS acceleration according to the NHNM model is shown as well. 
The VC-curves describe the general trend of the measured data in the frequency range in which they are defined, but the (site-specific) resonant behaviour is not captured correctly. Therefore, the VC-curves should only be used as a rule of thumb in the initial stages of the design. In this thesis, an extension of the VC-curves (over frequency) is used in chapter 6 to derive requirements for sensors.

\subsection{Evaluating disturbance effects}

As already mentioned in section 2.1, the effects of disturbances on the machine accuracy are to be reduced by the vibration isolation system. In order to evaluate the effectiveness of the vibration isolation system, an appropriate performance measure has to be defined.

Denoting the error vector by $z(t)$, the $p$-norm of $z(t)$ may be considered for this purpose, with an appropriate choice for $p$, see equation (2.1). Common choices for $p$ are 1 (integral absolute value), 2 (integral square value or "energy") and $\infty$ (peak value). Another common (semi-)norm is the RMS-norm, which is a measure for the average signal power, see equation (2.2).

$$
\begin{aligned}
& \|z(t)\|_{p}=\left(\int_{-\infty}^{\infty} \sum_{i=1}^{N_{z}}\left|z_{i}(\tau)\right|^{p} \mathrm{~d} \tau\right)^{1 / p}, \quad z(t) \in \mathbb{R}^{N_{z}} \\
& \|z(t)\|_{\mathrm{RMS}}=\lim _{T \rightarrow \infty} \sqrt{\frac{1}{2 T} \int_{-T}^{T} \sum_{i=1}^{N_{z}}\left|z_{i}(\tau)\right|^{2} \mathrm{~d} \tau}
\end{aligned}
$$

The RMS-norm has the advantage that its value is finite for signals with infinite energy, e.g. random signals and periodic signals. For finite energy signals, e.g. impact-like responses, the $p$-norms result in finite values but the RMS-norm is zero. In this thesis, the RMS-norm is adopted for random and periodic disturbances and the 2-norm for impact-like signals.

For linear, time-invariant (LTI) systems, the 2-norm of $z(t)$ can be expressed by equation (2.3), provided that the Fourier transform $\boldsymbol{W}(\omega)$ of the disturbance signal $w(t)$ exists. Here, $G(j \omega)$ is the multivariable frequency response function of the LTI system $G(s)$ and $\|.\|_{F}$ denotes the Frobenius norm.

$$
\begin{aligned}
\|z(t)\|_{2}^{2} & =\frac{1}{2 \pi} \int_{-\infty}^{\infty} \operatorname{tr}\left(\boldsymbol{Z}(\omega) \boldsymbol{Z}(\omega)^{\mathrm{H}}\right) \mathrm{d} \omega=\frac{1}{2 \pi} \int_{-\infty}^{\infty}\|\boldsymbol{Z}(\omega)\|_{F}^{2} \mathrm{~d} \omega \\
& =\frac{1}{2 \pi} \int_{-\infty}^{\infty}\|\boldsymbol{G}(j \omega) \boldsymbol{W}(\omega)\|_{F}^{2} \mathrm{~d} \omega
\end{aligned}
$$


For disturbance signals whose Fourier transforms do not exist, a similar result is found for the RMS-norm by using the power spectral density (PSD) matrix $\boldsymbol{P}_{w w}(\omega)$ of the disturbance signal, see equation (2.4).

$$
\|z(t)\|_{\mathrm{RMS}}^{2}=\frac{1}{2 \pi} \int_{-\infty}^{\infty} \operatorname{tr}\left(\boldsymbol{G}(j \omega) \boldsymbol{P}_{w w}(\omega) \boldsymbol{G}(j \omega)^{\mathrm{H}}\right) \mathrm{d} \omega
$$

When the disturbance signals are mutually independent, the power spectral density $\boldsymbol{P}_{w w}(\omega)$ is diagonal. In this case the RMS-norm can be expressed by equation (2.5).

$$
\|z(t)\|_{\mathrm{RMS}}^{2}=\frac{1}{2 \pi} \int_{-\infty}^{\infty}\left\|\boldsymbol{G}(j \omega) \sqrt{\boldsymbol{P}}_{w w}(\omega)\right\|_{F}^{2} \mathrm{~d} \omega
$$

In the early stages of the system design, it is desirable to use disturbance models instead of non-parametric PSD data. It is well known that coloured noise signals can be obtained by filtering white noise signals through a minimumphase LTI filter $\boldsymbol{\Phi}_{w}(s)$. $\boldsymbol{\Phi}_{w}(s)$ is commonly referred to as the spectral factor of $\boldsymbol{P}_{w w}(\omega)$, see equation (2.6) [Sayed and Kailath (2001)].

$$
\boldsymbol{P}_{w w}(\omega)=\boldsymbol{\Phi}_{w}(j \omega) \boldsymbol{\Phi}_{w}(j \omega)^{\mathrm{H}}
$$

Then, the RMS-norm is expressed in terms of the spectral factors of the disturbances and the frequency response function, as indicated in equation (2.7).

$$
\|z(t)\|_{\mathrm{RMS}}^{2}=\frac{1}{2 \pi} \int_{-\infty}^{\infty}\left\|\boldsymbol{G}(j \omega) \boldsymbol{\Phi}_{w}(j \omega)\right\|_{F}^{2} \mathrm{~d} \omega
$$

The interpretation of the 2-norm and the RMS-norm in terms of the frequency-dependent Frobenius norm is especially useful. Recall that the square of the Frobenius norm of a matrix equals the sum of the squares of the elements. Hence, this allows independent evaluation of the effect of each disturbance source. Moreover, this approach is very useful for determining in which frequency range a disturbance signal delivers the largest contribution to the error. It is the basis of Dynamic Error Budgeting (DEB), a system design approach put forward by [Jabben (2007); Jabben et al. (2008)].

\subsection{Basic system model}

In this section, a basic system model is introduced that still describes many of the relevant phenomena that occur in vibration isolation problems. This model is used often throughout this thesis to illustrate various theoretical results. The model is presented in figure 2.3. 


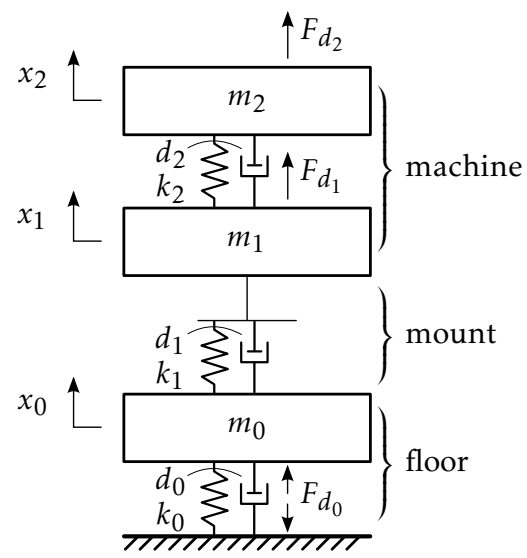

(a) Passive vibration isolation model

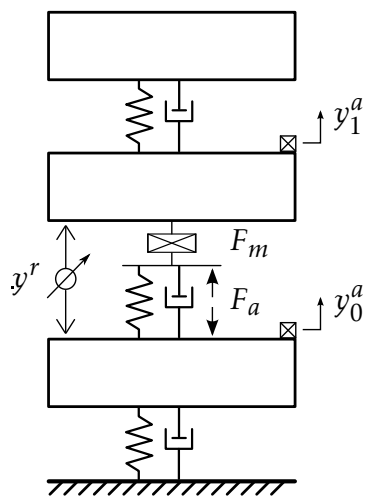

(b) Additional elements in the active vibration isolation model

Figure 2.3: Elementary models of vibration isolation problems

As shown in figure 2.3a, the model uses lumped masses, linear springs and viscous dampers to describe the dynamics of the machine, the mount and the floor. All bodies are restricted to translate only in one direction. The machine has one internal degree of freedom, which describes the internal deformation or structural mode. The mount is modelled as the parallel connection of the spring $k_{1}$ and the viscous damper $d_{1}$.

Given a certain total machine mass $\left(m_{1}+m_{2}\right)$, the stiffness $k_{1}$ determines the

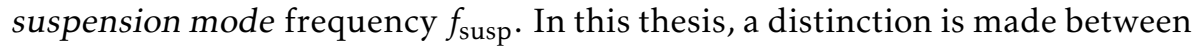
soft mount and hard mount systems. The division is, somewhat arbitrarily, chosen at $f_{\text {susp }}=5 \mathrm{~Hz}$, with soft mount systems having a suspension frequency smaller than this value and hard mount systems a higher one.

The floor or support structure ${ }^{2}$ is modelled as a mass-spring-damper system. Hence, the compliance of the floor, as well as its dominant vibration mode, can be taken into account. Several disturbance forces $\left(F_{d_{0}}, F_{d_{1}}, F_{d_{2}}\right)$ are shown that can excite the floor and the machine.

Figure $2.3 \mathrm{~b}$ shows the additional model elements that are relevant for active vibration isolation systems. These include a force actuator $F_{a}$ in the mount, as well as several types of sensors:

- $F_{m}$ : measurement of the force in the mount (assumed positive in compression);

- $y^{r}$ : measurement of relative motion between the machine and the floor (assumed positive in extension);

2 In general, the equipment is placed on the floor, but it may also be placed on a separate frame. Therefore, the support structure is comprised of everything not supported by the vibration isolation system. 
- $y_{0}^{a}$ : measurement of the floor motion with respect to an inertial reference (assumed positive in the indicated direction);

- $y_{1}^{a}$ : measurement of the machine motion with respect to an inertial reference and collocated with the mount (assumed positive in the indicated direction)

In this thesis, motion with respect to an inertial reference, i.e. $y_{0}^{a}$ and $y_{1}^{a}$, is referred to as absolute motion. Throughout this chapter, any absolute motion sensor is assumed to be ideal. Therefore, the absolute position, velocity and acceleration measurements can be readily converted back and forth by using analytical integration and differentiation.

However, it is well known that numerical differentiation of high-frequency signal components should be avoided in practice. Moreover, the bandwidth limitations of absolute motion sensors restrict the frequency range in which absolute motion measurements are available, see chapter 6 for details.

\subsubsection{Dynamic modelling}

Commonly, the machine response is described in terms of the displacement $x_{1}$ and the deformation $\Delta x \equiv x_{2}-x_{1}$. In the Laplace domain, the responses of these signals due to the three disturbance forces are expressed by six transfer functions: the compliances $C_{i}(s)$ of equation (2.8) and the deformabilities $D_{i}(s)$ of (2.9).

$$
\begin{array}{rlrl}
C_{0}(s) & \equiv \frac{X_{1}(s)}{F_{d_{0}}(s)}, & C_{1}(s) \equiv \frac{X_{1}(s)}{F_{d_{1}}(s)}, & C_{2}(s) \equiv \frac{X_{1}(s)}{F_{d_{2}}(s)} \\
D_{0}(s) \equiv \frac{\Delta X(s)}{F_{d_{0}}(s)}, & D_{1}(s) \equiv \frac{\Delta X(s)}{F_{d_{1}}(s)}, & D_{2}(s) \equiv \frac{\Delta X(s)}{F_{d_{2}}(s)}
\end{array}
$$

In practice, the disturbance source $F_{d_{0}}$ may be difficult to identify, and even, in many cases, there will be more than one source causing floor vibrations. As an alternative, the floor motion $x_{0}$ is usually considered to be an independent input signal, even though this is fundamentally incorrect. However, if the floor dynamics and machine dynamics are sufficiently decoupled, the approximation is valid. Then, the system response to floor motion is defined by the transmissibility $T(s)$ and the deformation transmissibility $T_{d}(s)$, see equation (2.10). Please note that $\ddot{X}_{i}(s)$ is a shorthand notation for the Laplace transformation of the acceleration signal $\ddot{x}_{i}(t)$.

$$
T(s) \equiv \frac{\ddot{X}_{1}(s)}{\ddot{X}_{0}(s)}, \quad T_{d}(s) \equiv \frac{\Delta X(s)}{\ddot{X}_{0}(s)}
$$

The reader is referred to appendix A.1 for the derivation of these transfer functions and their dependency on the model parameters. 


\subsubsection{Parameter values}

Table 2.1 lists the numerical values for the model parameters that are used throughout this chapter. These values represent the experimental setup that is used for control experiments. This setup is presented in chapter 7.

There are two settings for the parameter set $\left(k_{1}, d_{1}\right)$. These are chosen such that the suspension mode frequency is either $1 \mathrm{~Hz}$ or $10 \mathrm{~Hz}$, i.e. either a soft mount or a hard mount support is obtained. The soft mount parameters are indicated by a superscripted $s$ and the hard mount parameters by a superscripted $h$.

The value for $k_{2}$ is chosen such that the structural mode frequency is approximately $100 \mathrm{~Hz}$. The damping values are chosen such that the suspension and structural modes are approximately 1\% damped and the floor vibration mode is approximately $10 \%$ damped.

Table 2.1: Numerical values for model parameters, see figure 2.3

\begin{tabular}{|c|c|c|c|c|c|}
\hline Param. & Value & & Value & & Value \\
\hline$m_{0}$ & $20 \mathrm{~kg}$ & $d_{0}$ & $120 \mathrm{Ns} / \mathrm{m}$ & $k_{0}$ & $20 \cdot 10^{3} \mathrm{~N} / \mathrm{m}$ \\
\hline$m_{1}$ & $2.5 \mathrm{~kg}$ & $d_{1}^{s}$ & $0.5 \mathrm{Ns} / \mathrm{m}$ & $k_{1}^{s}$ & $0.2 \cdot 10^{3} \mathrm{~N} / \mathrm{m}$ \\
\hline & & $d_{1}^{h}$ & $2.5 \mathrm{Ns} / \mathrm{m}$ & $k_{1}^{h}$ & $20 \cdot 10^{3} \mathrm{~N} / \mathrm{m}$ \\
\hline$m_{2}$ & $2.5 \mathrm{~kg}$ & $d_{2}$ & $15 \mathrm{Ns} / \mathrm{m}$ & $k_{2}$ & $500 \cdot 10^{3} \mathrm{~N} / \mathrm{m}$ \\
\hline
\end{tabular}

\subsection{Passive vibration isolation}

In this section, the effects of the mechanical design parameters on the vibration isolation performance are examined. The focus lies on the mechanical parameters of the mount, i.e. $k_{1}$ and $d_{1}$, but the effect of the machine parameters $\left(m_{1}, m_{2}, d_{2}, k_{2}\right)$ on the machine's sensitivity to the various disturbance sources is also discussed briefly.

Moreover, some basic design guidelines for the machine parameters $\left(m_{1}, m_{2}\right.$, $d_{2}, k_{2}$ ) are derived in section A.1.3 which result in a reduced sensitivity to vibration sources. The main conclusion of section A.1.3 is repeated here:

In order to reduce the sensitivity to most disturbance sources, a lightweight, stiff upper structure design is preferred, with sufficient internal damping and placed on a heavy (rigid) base frame.

Especially the latter two requirements may be difficult or impractical to realize in practice, when only passive means are used. 


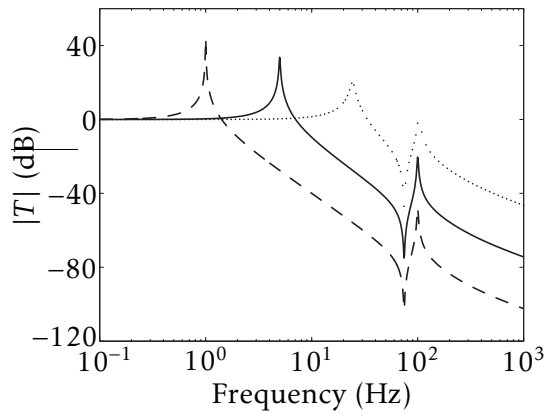

(a) Effect of $k_{1}$ on $T(s)$

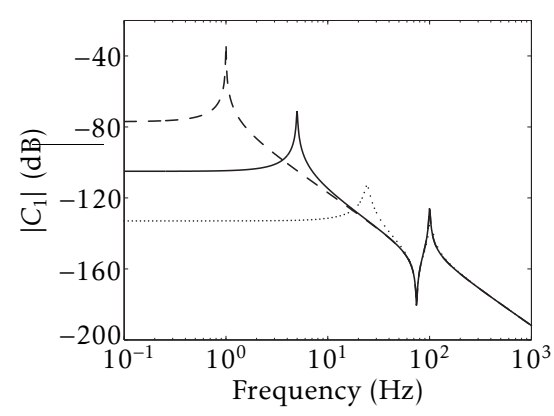

(b) Effect of $k_{1}$ on $C_{1}(s)$

Figure 2.4: Effect of $k_{1}$ on the transmissibility $T(s)$ and the compliance $C_{1}(s)$. The value of $k_{1}$ was varied by a factor 25 , resulting in suspension frequencies of $1 \mathrm{~Hz}$ (dashed), $5 \mathrm{~Hz}$ (solid) and $25 \mathrm{~Hz}$ (dotted).

Of more interest for a vibration isolation system design are the effects of the support parameters $k_{1}$ and $d_{1}$. To illustrate these effects, the transmissibility $T(s)$ and the compliance $C_{1}(s)$ are examined more closely. The expressions for these transfer functions are derived in appendix A.1.2 and are repeated in equations (2.11) and (2.12).5

$$
\begin{aligned}
T(s) & \equiv \frac{\ddot{X}_{1}(s)}{\ddot{X}_{0}(s)}=\frac{\left(k_{1}+d_{1} s\right)\left(m_{2} s^{2}+d_{2} s+k_{2}\right)}{p_{\mathrm{r}}(s)} \\
C_{1}(s) & \equiv \frac{X_{1}(s)}{F_{d_{1}}(s)}=\frac{m_{2} s^{2}+d_{2} s+k_{2}}{p_{\mathrm{r}}(s)} \\
p_{\mathrm{r}}(s) & =\left(m_{1} s^{2}+\left(d_{1}+d_{2}\right) s+k_{1}+k_{2}\right)\left(m_{2} s^{2}+d_{2} s+k_{2}\right)-\left(k_{2}+d_{2} s\right)^{2}
\end{aligned}
$$

Figure 2.4a illustrates the effect of increasing support stiffness on the transmissibility $T(s)$, resulting in a suspension frequency $f_{\text {susp }}$ of $1 \mathrm{~Hz}$ (dashed), $5 \mathrm{~Hz}$ (solid) and $25 \mathrm{~Hz}$ (dotted) respectively. The effect on the deformation transmissibility $T_{d}(s)$ is similar, i.e. an increase of the suspension frequency and an increase of the frequency response above the suspension frequency.

The effect on the compliance $C_{1}(s)$ is shown in figure $2.4 \mathrm{~b}$. In this case, the frequency response is reduced at frequencies below the suspension frequency. Similar effects occur in the compliance $C_{2}(s)$ and the deformability $D_{1}(s)$. There is no significant effect on the deformability $D_{2}(s)$.

From these figures, it is clear that a low support stiffness $k_{1}$ is preferred with regard to the transmissibility $T(s)$ (and $T_{d}(s)$ ), whereas a high support stiffness is more beneficial in terms of the compliance $C_{1}(s)$ (as well as $C_{2}(s)$ and $D_{1}(s)$ ). 
As a result of these conflicting requirements, the choice of $k_{1}$ offers a trade-off between floor vibration isolation and rejection of direct disturbances. Hence, that choice should be based on the (expected) relative importance of floor vibrations versus direct disturbance forces.

In most applications, floor vibrations are the major source of disturbances, and hence a soft mount system is preferred. Unfortunately, the low support stiffness in soft mount systems introduces several disadvantages compared to hard mount systems:

- direct disturbances induce larger displacements and deformations, as well as longer settling times

- the tilt modes of machines with a high centre of mass may become unstable

- the levelling problem due to gravity ${ }^{3}$

As an illustration of the levelling problem, equation (2.14) gives the static deflection $\Delta x$ of a body with mass $m$, which is supported by a linear spring with stiffness $k_{1}$ in Earth's gravity field. For a (typical) soft mount suspension resonance frequency of $1 \mathrm{~Hz}$, the static deflection will be $0.25 \mathrm{~m}($ !). In order to compensate for this deflection, soft mount systems have to be equipped with an additional levelling system.

$$
\Delta x=-\frac{m g}{k_{1}}=-\frac{g}{\left(2 \pi f_{\text {susp }}\right)^{2}}
$$

The second design trade-off in passive isolator design is concerned with the damping constant $d_{1}$. Figures 2.5a and 2.5b illustrate the effect of increasing $d_{1}$ (sequentially by a factor of 10 ) on the transmissibility and the compliance, resulting in suspension damping ratios of $1 \%$ (dashed), $10 \%$ (solid) and $100 \%$ (dotted) respectively.

In view of the compliance $C_{1}(s)$, increasing $d_{1}$ reduces the suspension resonance peak without any adverse effects. This is also the case for the other compliance $C_{2}(s)$ and the deformabilities $D_{1}(s)$ and $D_{2}(s)$.

The suspension resonance peak in the transmissibility response is also reduced. However, a larger damping constant $d_{1}$ results in increased transmissibility of floor vibrations in the frequency range above the suspension resonance frequency. More specifically, the $-40 \mathrm{~dB} /$ decade roll-off for undamped systems is converted into $-20 \mathrm{~dB} /$ decade roll-off. This is caused by the numerator term $\left(k_{1}+d_{1} s\right)$ in the transmissibility, see also equation (2.11). The same effect also occurs in the deformation transmissibility $T_{d}(s)$.

3 The levelling problem is actually related to the suspension frequency, and hence only indirectly to the support stiffness. See also equation (2.14) 


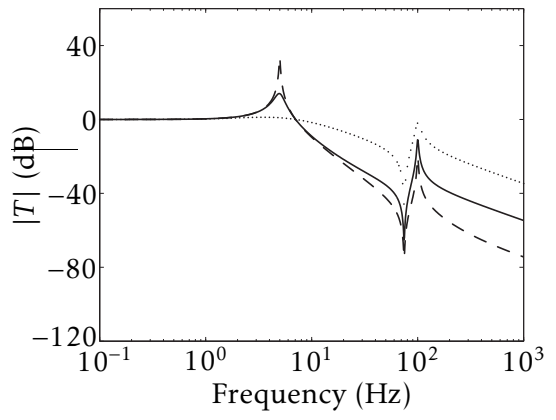

(a) Effect of $d_{1}$ on $T(s)$

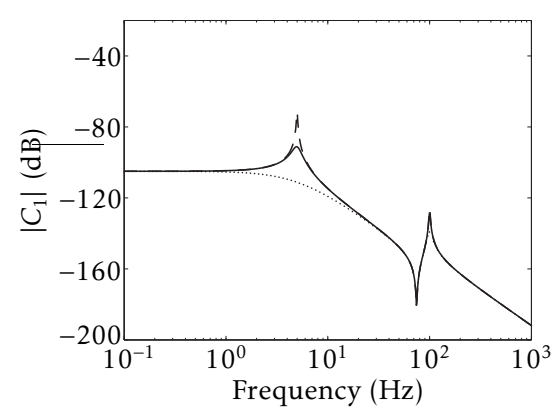

(b) Effect of $d_{1}$ on $C_{1}(s)$

Figure 2.5: Effect of $d_{1}$ on the transmissibility $T(s)$ and the compliance $C_{1}(s)$. The value of $d_{1}$ was varied by a factor 10, resulting in suspension damping ratios of 1\% (dashed), 10\% (solid) and 100\% (dotted).

\subsection{Active vibration isolation}

By augmenting the passive isolator with actuators, sensors and an associated control system, some of the drawbacks of passive vibration isolation systems can be overcome. Depending on the control strategy, active vibration isolation systems can be based on feedback control, feedforward control, or a combination of both. Feedback control can be used to change the eigenvalues (poles) of a system. As a result, the system response to all inputs can be altered (to some extent). On the other hand, feedforward control can only be used to improve the response to a specific set of inputs.

In the following sections, some basic feedback and feedforward control laws are presented. The main purpose of these sections is to illustrate how feedback and feedforward control can be used to improved various characteristics of the vibration isolation system.

\subsubsection{Active vibration isolation using feedback control}

Figure 2.6 shows a simplification of the basic system model of figure 2.3. The floor motion $x_{0}$ is regarded as an independent input. Moreover, the internal mode has been disregarded for the moment, as most state-of-the-art vibration isolation systems are primarily concerned with the suspension mode(s) of the machines. The viscous damper has also been left out. In the previous section, the disadvantage of high (relative) damping at frequencies above the suspension frequency was demonstrated. Therefore, active vibration isolation systems are usually designed with little or no mechanical damping. Instead, reduction of the suspension resonance peak is achieved by the feedback control system. 


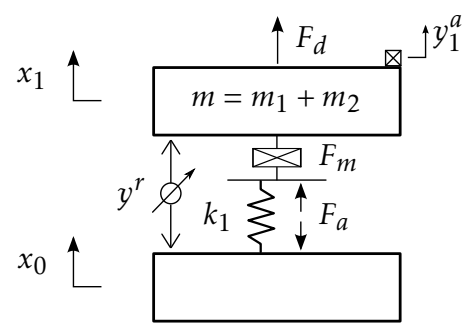

Figure 2.6: Model of a machine $m$ supported by an active vibration isolation system, which consists of a linear spring $k_{1}$, a feedback-controlled force actuator $F_{a}$ and several possible sensors $y_{1}^{a}, y^{r}, F_{m}$. The machine is subjected to a direct disturbance force $F_{d}$ and floor vibrations $x_{0}$.

Equation (2.15) expresses the system's equation of motion in the Laplace domain, assuming zero initial conditions. The measurement signals $Y_{1}^{a}(s), Y^{r}(s)$ and $F_{m}(s)$ are expressed in equations (2.16)-(2.18).

$$
\begin{aligned}
& \left(m s^{2}+k_{1}\right) X_{1}(s)=k_{1} X_{0}(s)+F_{d}(s)+F_{a}(s) \\
& Y_{1}^{a}(s)=X_{1}(s) \\
& Y^{r}(s)=X_{1}(s)-X_{0}(s) \\
& F_{m}(s)=F_{a}(s)-k_{1}\left(X_{1}(s)-X_{0}(s)\right)
\end{aligned}
$$

\section{Absolute motion measurement}

Initially, feedback control based on the absolute measurement of equation (2.16) is considered, see equation (2.19). The feedback controller combines proportional, derivative and double derivative control. The feedback parameters $k_{p}, k_{v}$ and $k_{a}$ are the absolute position, absolute velocity and absolute acceleration feedback gains.

$$
F_{a}(s)=-\left(k_{p}+k_{v} s+k_{a} s^{2}\right) X_{1}(s)
$$

The resulting closed loop equation of motion for this system is expressed in equation (2.20).

$$
\left(\left(m+k_{a}\right) s^{2}+k_{v} s+k_{1}+k_{p}\right) X_{1}(s)=k_{1} X_{0}(s)+F_{d}(s)
$$




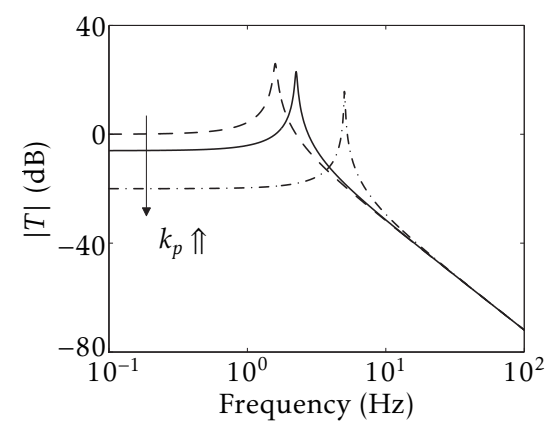

(a) Effect of $k_{p}$ on $T(s)$

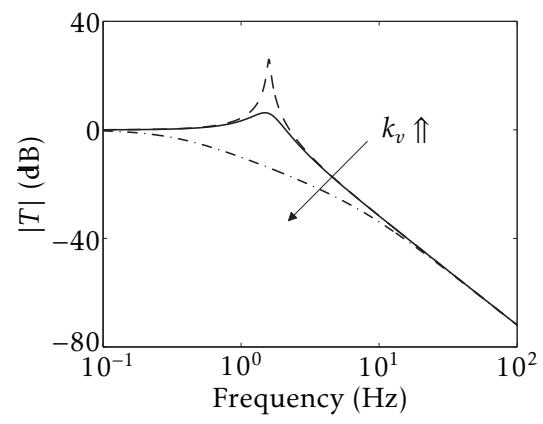

(b) Effect of $k_{v}$ on $T(s)$

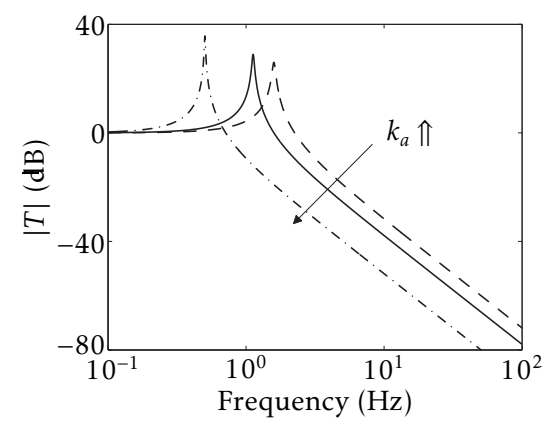

(c) Effect of $k_{a}$ on $T(s)$

Figure 2.7: Effect of the feedback parameters $k_{p}, k_{v}$ and $k_{a}$ on the magnitude frequency response of the transmissibility $T(s)$. The single arrows indicate an increase in the parameter value.

The corresponding transmissibility $T(s)$ and compliance $C_{1}(s)$ of this closed loop system are given in equations (2.21) and (2.22).

$$
\begin{aligned}
& T(s)=\frac{\ddot{X}_{1}(s)}{\ddot{X}_{0}(s)}=\frac{k_{1}}{\left(m+k_{a}\right) s^{2}+k_{v} s+k_{1}+k_{p}} \\
& C_{1}(s)=\frac{X_{1}(s)}{F_{d}(s)}=\frac{1}{\left(m+k_{a}\right) s^{2}+k_{v} s+k_{1}+k_{p}}
\end{aligned}
$$

From equation (2.20), it is clear that the feedback parameters $k_{p}, k_{v}$ and $k_{a}$ can be used to add (servo) stiffness, damping and mass to the system. Figure 2.7 illustrates these effects of the feedback parameters on the closed loop transmissibility. Note that the transmissibility and compliance are now identical, up to scaling by the support stiffness $k_{1}$, and hence the same effects apply to the closed loop compliance. 
Through the position feedback parameter $k_{p}$, the stiffness and the suspension frequency can be increased. From figure 2.7a, it appears that even the static transmissibility can be reduced. This is however a theoretical result. In practice, absolute motion sensors are AC-coupled, i.e. they have zero response to low frequency displacements.

The velocity feedback parameter $k_{v}$ provides an inertial damping force (proportional to the absolute machine velocity). This is commonly called skyhook damping, a term introduced by [Crosby and Karnopp (1973)]. As shown in figure $2.7 \mathrm{~b},-40 \mathrm{~dB} /$ decade high-frequency roll-off and damping at resonance can be obtained simultaneously with ideal skyhook damping. For sufficiently high velocity feedback gains, even overcritical damping can be achieved.

The acceleration feedback gain $k_{a}$ adds virtual mass. As a result, it can be used to reduce the closed loop suspension frequency, see figure $2.7 \mathrm{c}$.

\section{Relative motion measurement}

Now, consider the feedback control law of equation (2.23). This control law also combines proportional, derivative and double derivative control, but uses the relative motion measurement signal $Y^{r}(s)$.

The resulting closed loop equation of motion for this system is expressed in equation (2.24). The corresponding transmissibility $T(s)$ and compliance $C_{1}(s)$ of this closed loop system are given in equations (2.25) and (2.26).

$$
\begin{aligned}
& F_{a}(s)=-\left(k_{p}+k_{v} s+k_{a} s^{2}\right) Y^{r}(s) \\
& \left(\left(m+k_{a}\right) s^{2}+k_{v} s+k_{1}+k_{p}\right) X_{1}(s)=\left(k_{a} s^{2}+k_{v} s+k_{1}+k_{p}\right) X_{0}(s)+F_{d}(s) \\
& T(s)=\frac{k_{a} s^{2}+k_{v} s+k_{1}+k_{p}}{\left(m+k_{a}\right) s^{2}+k_{v} s+k_{1}+k_{p}} \\
& C_{1}(s)=\frac{1}{\left(m+k_{a}\right) s^{2}+k_{v} s+k_{1}+k_{p}}
\end{aligned}
$$

From both equations (2.25) and (2.26), it is concluded that the position feedback parameter $k_{p}$ is equivalent to a mechanical stiffness parallel to $k_{1}$. Hence, $k_{p}$ can be used to increase the suspension stiffness (and the suspension resonance frequency).

The velocity feedback parameter $k_{v}$ appears in exactly the same places as the mechanical parameter $d_{1}$ in equation (2.11). Therefore, the velocity feedback is equivalent to a mechanical damper placed between the machine and the floor. As a result, skyhook damping can not be achieved using this feedback control law. 
However, the $-40 \mathrm{~dB} /$ decade roll-off of the transmissibility can be retained by applying a frequency-dependent velocity feedback, see equation (2.27) for an example. Unfortunately, it can be shown that the suspension frequency will increase simultaneously.

$$
\bar{k}_{v}(s)=\frac{k_{v}}{1+\tau_{v} s}
$$

The acceleration feedback $k_{a}$ can be used to reduce the suspension resonance frequency. However, from equation (2.25), it is clear that it also eliminates the $-40 \mathrm{~dB} /$ decade roll-off of the transmissibility $T(s)$ at high frequencies. Again, this may be avoided by using a frequency-dependent acceleration feedback, see for example equation (2.28).

$$
\bar{k}_{a}(s)=\frac{k_{a}}{1+2 \zeta_{a} \tau_{a} s+\tau_{a}^{2} s^{2}}
$$

Another possibility is to include integral feedback. In this case, the static compliance will be zero. As a result, the machine displacement to constant force disturbances is zero. Hence, the levelling problem of (soft mount) vibration isolation systems can be solved through integral feedback of relative position measurement.

\section{Force measurement}

Combining the equation of motion (2.15) with the definition (2.18) of the force measurement $F_{m}(s)$ leads to an alternative expression for the measured force, as indicated in equation (2.29).

$$
\begin{aligned}
& F_{m}(s)=m s^{2} X_{1}(s)-F_{d}(s) \\
& F_{a}(s)=-\left(\frac{k_{v}}{s}+k_{a}\right) F_{m}(s)=-\left(\frac{k_{v}}{s}+k_{a}\right)\left(m s^{2} X_{1}(s)-F_{d}(s)\right)
\end{aligned}
$$

When the proportional-integral feedback control law of equation (2.30) is applied, the closed loop equation of motion is given by equation (2.31).

$$
\left(m\left(1+k_{a}\right) s^{2}+m k_{v} s+k_{1}\right) X_{1}(s)=k_{1} X_{0}(s)+\left(1+k_{a}+\frac{k_{v}}{s}\right) F_{d}(s)
$$


The corresponding transmissibility $T(s)$ and compliance $C_{1}(s)$ are given in equations (2.32) and (2.33).

$$
\begin{aligned}
& T(s)=\frac{k_{1}}{m\left(1+k_{a}\right) s^{2}+m k_{v} s+k_{1}} \\
& C_{1}(s)=\frac{\left(1+k_{a}\right) s+k_{v}}{s} \frac{1}{m\left(1+k_{a}\right) s^{2}+m k_{v} s+k_{1}}
\end{aligned}
$$

In terms of the transmissibility, the force feedback is conceptually the same as absolute acceleration feedback (compare equations (2.21) and (2.32)), although the feedback parameters are now scaled by $m$. As a result, skyhook damping can also be achieved by integral force feedback (IFF).

However, for this case, the closed loop compliance shows a significantly different behaviour. In particular, the static compliance is infinite. Hence, the static stiffness as observed by a direct force disturbance is zero, causing serious levelling problems. Once more, this can be solved by using a frequencydependent velocity feedback parameter, which should contain a high-pass filter of at least first order, see equation (2.34). However, the compliance at low frequency is inevitably increased by this type of feedback control.

$$
\bar{k}_{v}(s)=\frac{s k_{v}}{1+\tau_{v} s}
$$

\section{Summary and additional remarks}

In this section, basic feedback laws based on absolute motion, relative motion and force measurements have been presented. In principle, each feedback law provides a means to add (servo) stiffness, damping and/or mass to the system. Adding servo stiffness is most useful for relative motion feedback. Especially integral relative motion feedback can be applied to eliminate the levelling problem. Moreover, adding servo stiffness is useful for soft mount systems with unstable tilt modes [Heertjes et al. (2005)].

Both the absolute motion and force measurements allow the implementation of skyhook damping, although this has an adverse effect on the effective stiffness in case of force feedback. When relative motion measurement is used, skyhook damping can not be achieved, but may be approximated by frequency-dependent (low-pass filtered) velocity feedback. In this case, the suspension frequency is increased simultaneously.

By adding virtual mass, the suspension frequency can be reduced without altering the support stiffness. This is beneficial for hard mount systems in particular. Both force feedback and absolute acceleration feedback can be used for this purpose. 
The feedback laws presented in this section are meant to illustrate some of the possibilities and limitations of feedback control for active vibration isolation. Obviously, more complex control laws can be conceived. Moreover, many of the feedback laws are improper, which means they can not be implemented in their current forms.

Lastly, some control laws result in infinite bandwidth closed loop systems. In practice, the bandwidth has to be limited due to additional system and/or sensor dynamics, which have been ignored so far. Outside the control bandwidth all the closed loop responses inevitably have to return to the open loop responses.

\subsubsection{Active vibration isolation using feedforward control}

Vibration isolation by feedforward control relies on compensating for specific disturbances, by generating forces which cancel the effects of the disturbances on the machine. Therefore, it is also known as vibration cancellation. Obviously, the disturbances have to be known in order to counteract their effects. This knowledge can be user-supplied or obtained from measurements. An example of the former are signals generated by a stage motion controller and this type of feedforward control is referred to as motion feedforward. In contrast, when the feedforward is based on measurements, it is referred to as vibration feedforward. Figure 2.8 illustrates the latter situation, in which floor motion measurements are used to control the actuator. In this thesis only vibration feedforward is considered.

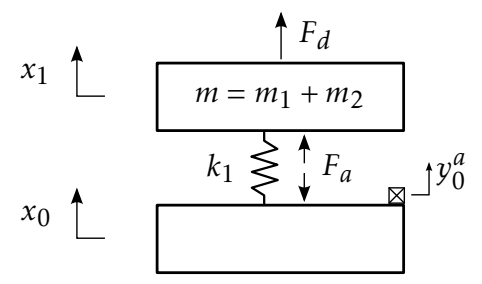

Figure 2.8: Model of a machine $m$ supported by an active vibration isolation system, which consists of a linear spring $k_{1}$, a feedforward-controlled force actuator $F_{a}$ and an absolute motion sensor $y_{0}^{a}$. The machine is subjected to a direct disturbance force $F_{d}$ and floor vibrations $x_{0}$.

For this system, the equation of motion is given by equation (2.35). The optimal feedforward control signal, which will compensate exactly for the effect of floor vibrations, is given by equation (2.36).

$$
\begin{aligned}
\left(m s^{2}+k_{1}\right) X_{1}(s) & =k_{1} X_{0}(s)+F_{d}(s)+F_{a}(s) \\
F_{a}(s) & =-k_{1} X_{0}(s)
\end{aligned}
$$


In this example, the feedforward control law is deceivingly simple. However, in general, it should be interpreted as an attempt to generate a counteracting force or anti-force. Therefore, every frequency component in the disturbing force should be matched in magnitude, but with opposite phase.

For more complex systems, the feedforward control law should not only compensate for the static stiffness, but for the dynamic stiffness. In this dynamic stiffness the effects of static stiffness, damping and structural dynamics are all combined. In chapter 5 , methods to find such a feedforward control law are discussed in detail.

\subsection{State of the art}

Most industrial vibration isolation systems fall into the soft mount system category, with the high-end isolators having suspension frequencies $f_{\text {susp }} \lesssim 1$ $\mathrm{Hz}$. In general, these industrial systems achieve -30 to $-40 \mathrm{~dB} /$ decade roll-off in the transmissibility function above the suspension frequency. At high frequencies, the lower transmissibility limit ranges from -35 to $-60 \mathrm{~dB}$ at best. See e.g. the websites of the following manufacturers [Halcyonics; Minus $\mathrm{K}$ Technology; Technical Manufacturing Corporation (TMC)]. Feedforward control is sometimes offered as an extension kit, and concerns motion feedforward in most cases.

Usually, these isolators use steel coil springs or pneumatic isolators for vertical isolation, whereas pendulum or parallel guidance mechanisms are used for horizontal isolation, see e.g. [Nelson (1998); Platus (1999)].

Several authors have reported the use of nonlinear springs [Winterflood et al. (2002)] or negative spring mechanisms [Platus (1999)] to overcome the levelling problem for soft mounts.

State-of-the-art soft mount isolation systems use feedback control to actively dampen the suspension resonance. For an excellent introduction to active damping in flexible structures, see the textbook by [Preumont (2002)].

Heertjes et al. use a modal feedback control approach to achieve active suspension damping, low-frequency levelling control as well as stabilization of tilting modes [Heertjes et al. (2005)].

Zuo et al. propose the use of sliding mode control to achieve skyhook suspension damping [Zuo et al. (2004); Zuo and Slotine (2005); Zuo et al. (2005)]. Their method is not restricted to skyhook damping, but also allows more general performance specifications.

Active hard mount vibration isolation systems are not common, although some experimental setups with hard mount characteristics have been reported, see e.g. [Nakamura et al. (2000); Redaelli et al. (2004)]. 
The only industrial system that is explicitly advertised to be a hard mount system is the TMC STACIS ${ }^{\circledR}$ system [Beard et al. (1994); Schubert et al. (1998)]. It uses a two-stage active/passive approach to reduce the transmissibility of floor vibrations. High gain feedback control of an intermediate mass, which is mounted very stiffly to the floor $\left(f_{\text {res }} \gg 10 \mathrm{~Hz}\right)$, provides a "quiet" platform for the second, passive isolation stage, which provides a suspension frequency of approximately $20 \mathrm{~Hz}$. For the STACIS system, motion feedforward is optional.

Vervoordeldonk et al. propose a soft mount vibration isolation system which uses position feedback control to force the machine to follow a separate reference mass [Vervoordeldonk et al. (2004, 2007)]. The reference mass is designed to be isolated from floor vibrations and direct disturbances, thus providing an inertial position reference above the suspension frequency of the reference mass. The position feedback control is then used to reduce the sensitivity to direct disturbances, without affecting the floor vibration isolation performance (or even improving it).

As mentioned earlier, most state-of-the-art vibration isolation systems do not specifically take into account the structural resonance modes which may be present in the supported machine. A device which specifically aims at actively increasing the damping of structural resonance modes is described by [Holterman et al. (2002)]. Moreover, it has been proposed recently to extend the feedback control of active vibration isolation mounts to increase the structural damping of selected modes in the supported machine [Vervoordeldonk et al. (2006)].

On a side note, vibration isolation problems are also encountered in space structure engineering. Although the performance requirements may differ considerably from Earth-based high-precision equipment, the same principles apply. The use of $\mathcal{H}_{2}$-control design for several active soft mount vibration isolation systems is described by [Hyde and Crawley (1995)]. The use of six degrees of freedom (DOF) Stewart platforms with various feedback control laws has been reported by e.g. [Spanos et al. (1995); Cobb et al. (1999); Hauge and Campbell (2004)]. The use of adaptive feedforward control for tonal vibration isolation using a Stewart platform is described by [Geng and Haynes (1994); Anderson and How (1997)]. 



\section{Chapter 3}

\section{Active hard mount concept}

In this thesis, it is investigated whether the inherent disadvantages of soft mount systems ${ }^{1}$ can be circumvented by using hard mounts instead. In this case, an active control system is required to improve the vibration isolation performance.

In section 3.1, several performance objectives for the hard mount vibration isolation system are discussed. For these systems, an active control system is a necessity. The chosen control strategy is discussed briefly in section 3.2.1. Then, the mechanical design concept is presented in section 3.2.2. In this section, the effect of non-ideal mechanical behaviour on the control performance is analysed. Based on the results of this theoretical analysis, guidelines for the mechanical design are formulated.

\subsection{Performance objectives}

As already mentioned in section 2.1, the general purpose of the vibration isolation system is to increase the machine accuracy. In the absence of a specific application, and hence a specific definition of accuracy, it is assumed that the internal deformation of the machine is the performance objective that should be minimized.

Recall from section 2.3 that, in terms of signal power, the overall internal deformation is a summation of the individual deformation responses to the various disturbance sources. In turn, each of these responses is determined (in the frequency domain) by the product of a frequency response function and the power spectral density of the respective disturbance source.

1 See page 16 


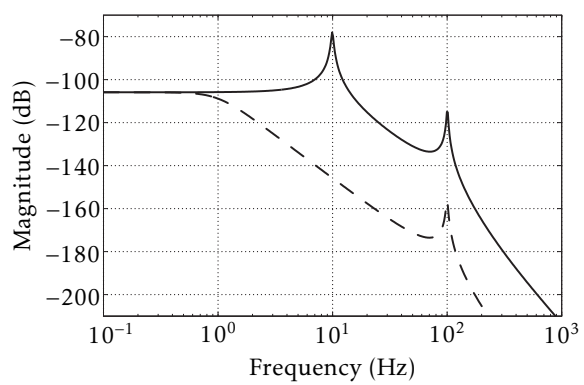

(a) Deformation transmissibility $T_{d}(s)=\Delta X(s) / \ddot{X}_{0}(s)$

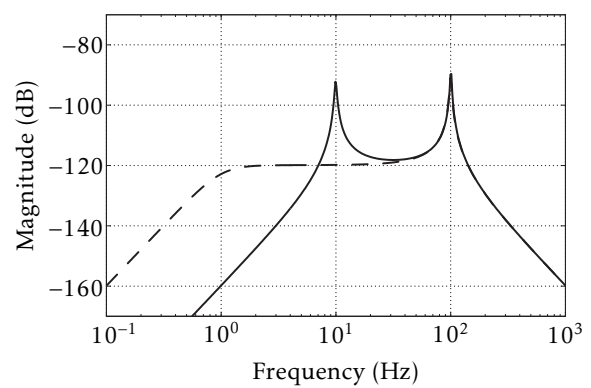

(b) Deformability $D_{1}(s)=\Delta X(s) / F_{d_{1}}(s)$

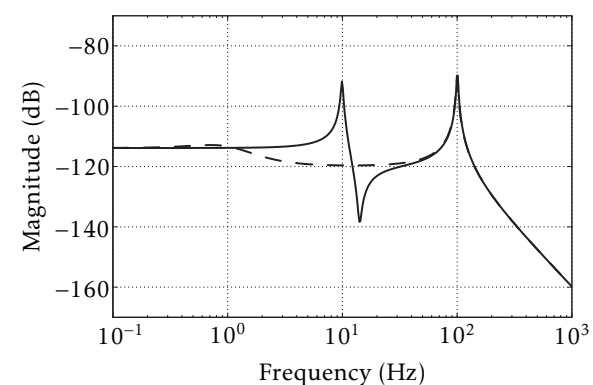

(c) Deformability $D_{2}(s)=\Delta X(s) / F_{d_{2}}(s)$

Figure 3.1: Deformation responses of the reference soft mount system (dashed) and a passive (10 Hz) hard mount system (solid) to: (a) floor acceleration $\ddot{x}_{0} ;(b)$ direct disturbance $F_{d_{1}} ;(c)$ direct disturbance $F_{d_{2}}$

Therefore, the frequency response functions to the various disturbance sources are important factors in the vibration isolation performance. In figure 3.1, the frequency response functions of the deformation $\Delta x$ due to floor vibrations $\ddot{x}_{0}$ and the direct disturbances $F_{d_{1}}$ and $F_{d_{2}}$ are compared for a passive hard mount system with its suspension frequency at $10 \mathrm{~Hz}$ and a reference soft mount system (1 Hz suspension frequency).

The underlying model for the hard mount system and the soft mount system has been presented in section 2.4. Moreover, it is assumed that the soft mount system is equipped with an active control system that achieves $70 \%$ skyhook damping of the suspension mode.

By comparing the passive hard mount response to the active soft mount response to floor vibrations (figure 3.1a), it is immediately clear that the response to floor vibrations has to be improved drastically for frequencies above the soft mount suspension frequency of $1 \mathrm{~Hz}$. 
In figure 3.1b, the increased robustness of the hard mount system to the direct disturbance force $F_{d_{1}}$ at low frequencies is observed clearly. However, the damping of the suspension resonance mode must be improved significantly to achieve an overall better performance.

From figure 3.1c, a similar conclusion is reached regarding the response of the hard mount system to the direct disturbance force $F_{d_{2}}$. It will only be similar to or smaller than the soft mount response, when the damping of the suspension resonance is improved significantly.

Finally, it is immediately clear in all these figures that increasing the damping of the structural mode is also desirable.

Based on these observations, the following qualitative performance objectives for active hard mount vibration isolation systems can be formulated:

\section{i. High support stiffness:}

A relatively high support stiffness improves the robustness to lowfrequency (and static) direct disturbances like e.g. stage acceleration forces and forces transmitted by cables. Moreover, the difficulties related to levelling are reduced.

ii. Small deformation transmissibility:

The passive response of a hard mount system to floor vibrations usually offers insufficient vibration isolation. Therefore, the effect of floor vibrations on the internal deformation must be reduced. This requires an active vibration isolation system.

iii. Skyhook damping of the suspension mode:

Recall from figure 2.5 that mechanical (viscous) damping in the mount increases the transmissibility of floor vibrations at high frequency. Therefore, many isolators are designed with little mechanical damping. The suspension damping must then be increased by using feedback control. Preferably, skyhook damping must be achieved.

iv. Improved damping of the relevant structural modes:

It is clear from figure 3.1 that poorly damped structural resonance modes can have a significant contribution to the structural deformation. For hard mount systems, this is especially the case when regarding floor vibrations (see figure 3.1a). Therefore, the active vibration isolation system must be able to improve the damping of these relevant structural modes.

As mentioned in section 2.1, the quantitative objectives depend heavily on the application (the required accuracy level) and the environment (disturbance levels). This is most obvious for the support stiffness, which offers a tradeoff between robustness to direct disturbances and isolation of floor vibrations (see also figure 2.4). 
In this thesis, it is assumed that a relatively high support stiffness is required to cope with direct disturbances and reduce the levelling problem. With this high support stiffness being realized by the mechanical design, the remaining requirements, i.e. sufficiently high damping ratios for the suspension and relevant structural modes as well as reduction of the floor vibration transmissibility, can only be met by using active control. The remainder of this thesis will focus on active control methods for meeting these requirements.

Throughout this thesis, the following quantitative objectives for the control system are assumed:

\section{- Deformation transmissibility:}

The deformation transmissibility $T_{d}(s)$ and the transmissibility $T(s)$ have roughly the same frequency response for frequencies below the structural resonance frequency, aside from a scale factor (see appendix A, figures A.2a and A.2b). Therefore, an objective for the transmissibility function is stated instead. In chapter 6 , this transmissibility objective will also be used to determine requirements for sensors and actuators.

The desired floor vibration transmissibility is chosen as the transmissibility of a reference soft mount system, which is meant to represent a state-of-the-art vibration isolation system. The suspension frequency of this reference system is chosen at $1 \mathrm{~Hz}$. Moreover, $70 \%$ skyhook damping of the suspension mode is assumed (by means of an active control system). As mentioned in section 2.7, the transmissibility of such state-of-the-art systems typically has a lower limit between -35 and $-60 \mathrm{~dB}$ at best. In the remainder of the thesis, a lower transmissibility limit of $-60 \mathrm{~dB}$ will be assumed.

The reference transmissibility $T_{\text {ref }}(s)$ is then expressed by equation (3.1), with $\omega_{\text {susp,ref }}=2 \pi \mathrm{rad} / \mathrm{s}, \zeta_{\text {susp,ref }}=0.70$ and $\epsilon_{\text {ref }}=1 \cdot 10^{-3}$.

$$
T_{\text {ref }}(s)=\frac{\omega_{\text {susp,ref }}^{2}}{s^{2}+2 \zeta_{\text {susp,ref }} \omega_{\text {susp,ref }} s+\omega_{\text {susp,ref }}^{2}}-\epsilon_{\text {ref }}
$$

\section{- Suspension mode damping:}

The damping in the suspension mode is to be increased to at least $40 \%$ and should be achieved by skyhook damping.

- Structural mode damping:

The damping of the (relevant) structural mode(s) is to be increased to at least $10 \%$. 
Please note that the first objective is meant primarily to compare the active hard mount vibration isolation performance to state-of-the-art soft mount systems. It should not be interpreted as a strict performance target. On the other hand, the two objectives regarding the improved damping are meant as strict performance targets.

\subsection{Conceptual system design}

In the previous section, it is concluded that the hard mount isolation system most likely requires an active control system to improve the vibration isolation performance. In section 3.2.1, the chosen active control strategy is briefly introduced. Then, the mechanical design concept is discussed, in particular the effect of the mechanical design on the control performance. From this analysis, several guidelines for the mechanical design of hard mount isolators are derived.

\subsubsection{Control strategy}

From section 3.1, it is concluded that the active control system must add skyhook damping to the suspension mode, increase the damping of relevant structural modes and improve the floor vibration transmissibility, preferably to a level comparable to a state-of-the-art soft mount system.

Recall from section 2.6.1 that either proportional-integral (PI) control using force or acceleration measurement or proportional-derivative (PD) control using absolute velocity measurement can be used to add skyhook damping to the suspension mode, and simultaneously reduce the suspension mode frequency. Hence, these feedback laws and their respective sensor types are candidates for application in the considered hard mount concept.

However, the feedback control should also improve the damping in the structural mode. It turns out that these three requirements (increased suspension damping, increased structural damping and reduced suspension frequency) can not be met simultaneously by a single feedback controller. This will become clear in chapter 4 , where the feedback control design is discussed in detail. For the moment, it is only important to realize that some additional control system is required.

One possibility is to augment the supported machine with an internal control system which specifically targets the problem of structural damping. Such a system has been reported by e.g. [Holterman (2002)]. However, this requires additional hardware components to be incorporated into the design of the machine itself. This may not always be possible or practical. 
In this thesis, an attempt is made to achieve all the vibration isolation requirements by using active mounts only, i.e. without making changes to or adding active components to the supported machine. Therefore, all the required sensors and actuators are incorporated into the hard mount, resulting in a modular design.

In this case, the damping of the suspension mode and the (relevant) structural modes has to be achieved by feedback control. Then, the transmission of floor vibrations will be reduced by adding feedforward compensation of the measured floor vibrations. The feedback control strategy is discussed in detail in chapter 4 and the chosen feedforward control strategy is the subject of chapter 5 .

\subsubsection{Mechanical design concept}

The concept considered in this thesis, is based on an exact-constraint design. The notion of exact-constraint design is well known in precision engineering, see e.g. [Slocum (1992); Koster (1998); Blanding (1999)]. It is perhaps described best by [Hale (1999)]:

It is the objective of exact-constraint design to achieve some desired freedom of motion or perhaps no motion by applying the minimum number of constraints required.

When multiple constraints are applied to the same degree of freedom (DOF), it is said to be overconstrained. Usually, the same is said of the entire mechanism or object.

If a mechanism is overconstrained, support deformations, thermal loads and geometry variations due to assembly and part tolerances will induce internal stresses. These stresses and the accompanying deformations may become unacceptably large. Moreover, the induced deformations are difficult to predict. Therefore, an exact-constraint mechanical design is a necessity for a hard mount vibration isolation system, as the stiffnesses involved are rather large.

For our purpose, all rigid body (quasi-static) motion of the machine should be constrained. As any rigid body has six DOFs in three-dimensional space, six mutually independent constraints have to be applied.

In the considered hard mount concept, these six ideal single-DOF constraints are realized by six "legs". Each leg should be designed to be relatively stiff in one direction, called its principal direction, and very compliant in all other directions, called the parasitic directions.

The legs can be arranged in a hexapod configuration to achieve mutually independent axes, although more conventional configurations (e.g. legs aligned with Cartesian axes) are possible as well. 


\section{Planar example of the exact-constraint hard mount concept}

The exact-constraint hard mount concept is illustrated for a two-dimensional machine in figure 3.2a. The machine is assumed to be rigid, with mass $m$ and rotational inertia $J$, evaluated at the centre of mass $(C O M)$.

The machine has three degrees-of-freedom, which are exactly constrained by the three supporting legs (indicated by the spring elements and hinges). The model for each of the supporting legs is shown in more detail in figure $3.2 \mathrm{~b}$.

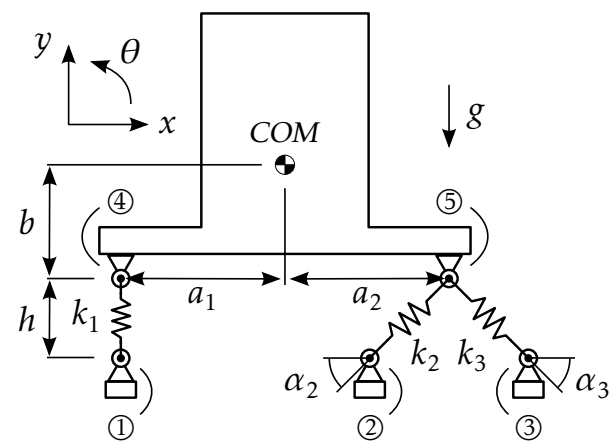

(a) Planar model of an exactly constrained, rigid machine

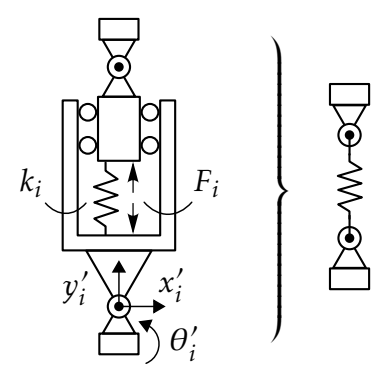

(b) Detailed leg model

Figure 3.2: Illustration of the exact-constraint design concept

Each leg is modelled by two hinges and a slider truss with internal stiffness $k_{i}$. A force actuator $F_{i}$ is incorporated in each leg for active control. The hinges are ideal, i.e. their rotational stiffness is zero. The slider truss only has a finite stiffness along its principal direction $y_{i}^{\prime}$ and is rigid in all other directions. As a result, each leg only provides stiffness in the principal direction $y_{i}^{\prime}$, but is completely free in any other direction, i.e. each leg provides exactly one constraint (when the internal stiffness is large enough).

The floor is modelled by three separate blocks. Each block can move and rotate independently, providing 27 rheonomic constraints, i.e. the accelerations, velocities and positions of each block and in each direction $\left(x_{i}^{\prime}, y_{i}^{\prime}, \theta_{i}^{\prime}\right)$ are prescribed. However, these constraints are not fully independent. For each block, the acceleration, velocity and position in each direction are interdependent through integral/derivative relations. Therefore, only nine independent driving conditions exist. In this section, the floor motion is expressed in terms of the nodal accelerations $\left(\ddot{x}_{i}^{\prime}, \ddot{y}_{i}^{\prime}\right)$ of the hinges (1)-(3) and the angular accelerations $\ddot{\theta}_{i}^{\prime}$ of the corresponding blocks. Note that the nodal displacements are expressed in the local coordinate systems of the legs. 
The design parameters for the legs are the stiffness parameters $k_{1}, k_{2}$ and $k_{3}$ and the geometry parameters $\alpha, \beta, a_{1}, a_{2}$ and $b$. Together, these parameters allow full control over the stiffness matrix of this system. As a result, the support stiffness in each direction and the suspension mode frequencies can, in principle, be tuned to any desired values.

Using this planar model, several guidelines for the mechanical design of hard mounts can be derived. For this purpose, a numerical example is evaluated, see table 3.1 for the chosen parameter values.

Table 3.1: Numerical values for model parameters of figure 3.2a

\begin{tabular}{|c|c|}
\hline Parameter & Value \\
\hline$a_{1}=a_{2}$ & $0.2 \mathrm{~m}$ \\
\hline$b$ & $0.035 \mathrm{~m}$ \\
\hline$h$ & $0.05 \mathrm{~m}$ \\
\hline$\alpha_{2}=\alpha_{3}$ & $45^{\circ}$ \\
\hline$k_{1}=k_{2}=k_{3}$ & $1 \cdot 10^{5} \mathrm{~N} / \mathrm{m}^{\mathrm{a}}$ \\
\hline$m$ & $12 \mathrm{~kg}$ \\
\hline$J$ & $2.2 \mathrm{~kg} \mathrm{~m}{ }^{2}$ \\
\hline \multicolumn{2}{|c|}{$\begin{array}{c}\text { Each leg also provides } 50 \mathrm{Ns} / \mathrm{m} \\
\text { of viscous damping }\end{array}$}
\end{tabular}

The equations of motion for this model can be derived in a parametric form from the Euler-Lagrange equations. However, even for this relatively simple system, the derivation is tedious and error prone. Instead, the model has been analysed using SPACAR, a nonlinear finite element software package for (flexible) multibody dynamics, see e.g. [Jonker et al. (2009)] and the references therein.

The nonlinear element deformation description used by SPACAR allows the accurate modelling of the system dynamics with relatively few elements. Moreover, rigid and flexible elements can be used simultaneously. Geometrical nonlinear stiffness effects like preloading due to gravity are easily included into the model as well.

Another advantage is that linearized state-space representations for arbitrary inputs and outputs can be easily computed. These state-space systems are of low order due to the relatively small number of elements compared to typical finite element models. Therefore, these system descriptions are easily used for analysis and control system design. 


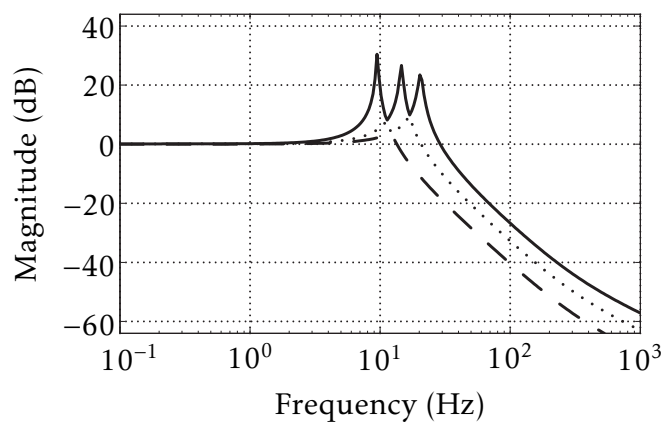

Figure 3.3: Singular value plot of the MIMO transmissibility for the model of figure 3.2a (see table 3.1 for parameter values). Inputs: see equation (3.2); outputs: see equation (3.3); $\quad-\sigma_{1}=\bar{\sigma} ; \quad \cdots: \sigma_{2} ; \quad--: \sigma_{3}$

With the numerical values as listed in table 3.1, the displacements ${ }^{2}$ of the centre of mass (COM) due to gravity are $0.47 \mu \mathrm{m}$ and $-0.59 \mathrm{~mm}$ in the $x$ - and $y$-direction. The resulting rotation is $-13 \mu \mathrm{rad}$.

The suspension frequencies of the linearized model around this equilibrium position are 9.6, 14.6 and $20.5 \mathrm{~Hz}$, respectively. The first two mode shapes exhibit a rotation and horizontal translation of the COM. The first mode is mostly dominated by rotation, the second by horizontal translation. The third mode shape is dominated by vertical translation.

To assess the floor vibration isolation performance for this multiple input, multiple output (MIMO) system, the singular value plot of the MIMO transmissibility is studied. This singular value plot is the extension of the Bode magnitude plot to MIMO systems. The largest singular value $\bar{\sigma}(f)$ is especially interesting for vibration isolation problems, as it is a measure of the worst-case vibration transfer as a function of frequency.

Careful selection and scaling of the input signals $\boldsymbol{u}$ and the output signals $y$ greatly improves the interpretation of the singular value plot. The selected input signals are the (local) $x$ - and $y$-accelerations and the scaled angular accelerations of the nodal points (1)-(3), see equation (3.2) and figure 3.2b. The output signals are described in terms of the (global) horizontal and vertical acceleration of the hinges (4) and (5), see equation (3.3) and figure 3.2a.

$$
\begin{aligned}
& \boldsymbol{u}=\left[\begin{array}{lllllllll}
\ddot{x}_{1}^{\prime} & \ddot{y}_{1}^{\prime} & h \ddot{\theta}_{1}^{\prime} & \ddot{x}_{2}^{\prime} & \ddot{y}_{2}^{\prime} & h \ddot{\theta}_{2}^{\prime} & \ddot{x}_{3}^{\prime} & \ddot{y}_{3}^{\prime} & h \ddot{\theta}_{3}^{\prime}
\end{array}\right]^{\mathrm{T}} \\
& y=\left[\begin{array}{lll}
\ddot{y}_{4} & \ddot{x}_{5} & \ddot{y}_{5}
\end{array}\right]^{\mathrm{T}}
\end{aligned}
$$
2 Displacement with respect to the initial configuration and aligned with the global coordinate
system as shown in figure 3.2a. 
In figure 3.3, the resulting singular value plot, representing the MIMO transmissibility, is shown for this example. Note that, due to the chosen input and output scaling, the transmissibility at low frequencies equals one $(0 \mathrm{~dB})$, similar to the single input, single output (SISO) transmissibility (see e.g. figure 2.4). The three suspension frequencies are clearly observed in the plot for the worst-case transmissibility $\bar{\sigma}(f)$.

Moreover, observe that the worst-case transmissibility does not roll-off until after the largest suspension frequency. If this largest suspension frequency is larger than strictly necessary, the task for the active control system (reducing the transmission of floor vibrations) is made more challenging without a good reason.

As the required high support stiffness puts a lower limit on the suspension frequencies, it naturally follows that the mechanical support should be designed to group all the suspension resonances relatively closely in frequency. This grouping of the suspension frequencies can be achieved by tuning the locations, orientations and stiffness properties of the mounts.

\subsubsection{Effects of non-ideal constraints}

The legs of the planar model of figure 3.2b are ideal, i.e. they provide only stiffness in their principal directions. In practice, the behaviour of such an ideal leg can only be approximated. To preserve the exact-constraint behaviour of the design, the ratio(s) of the parasitic stiffness to the principal stiffness should be sufficiently small. As it turns out, this is not only relevant for the realization of the desired suspension mode frequencies, but also for the performance limits of the active control system.

The mechanical design of the mount is not the only source of parasitic stiffness. Sensor and cooling water cables can also cause parasitic stiffness effects, see e.g. [Hauge and Campbell (2004)].

In this section, a method is presented for testing whether the parasitic stiffnesses are indeed sufficiently small. The method is illustrated by analysing an augmented version of the planar model of figure 3.2a, including parasitic stiffness. For this purpose, the hinges in each leg (six in total) are given a torsional stiffness $c_{\theta}$ of $1 \mathrm{Nm} / \mathrm{rad}$. For the vertical (leftmost) leg, this amounts to an equivalent transverse stiffness of $800 \mathrm{~N} / \mathrm{m}$, which is $0.8 \%$ of the longitudinal stiffness. For each of the slanted (rightmost) legs, this ratio is $0.4 \%$. The difference is caused by the different lengths of the legs.

In figure 3.4, the thick solid line shows the results of the singular value analysis for this augmented system. (The meaning of the other lines will be discussed in the following section.) There is no discernible difference compared to the ideal behaviour, compare figure 3.3. It can therefore be concluded that the exact-constraint requirements are still met for the evaluated value of the 


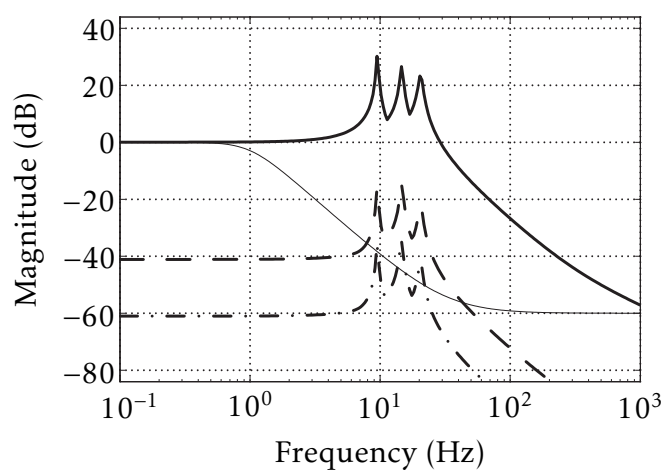

Figure 3.4: Largest singular value $\bar{\sigma}$ of the MIMO transmissibility for the augmented model (with parasitic stiffness $c_{\theta}$ ).

Inputs: see equation (3.2); outputs: see equation (3.3).

- $: c_{\theta}=1 \mathrm{Nm} / \mathrm{rad}$, without feedforward compensation (FF);

$--: c_{\theta}=1 \mathrm{Nm} / \mathrm{rad}$, with FF;

$-\cdot-: c_{\theta}=0.1 \mathrm{Nm} / \mathrm{rad}$, with FF;

- : soft mount reference transmissibility

parasitic stiffness (approximately 0.5\% of the principal stiffness).

However, non-ideal constraints may have more pronounced effects on the achievable vibration isolation performance when an active control system is present. This is examined in more detail in the following subsections.

\section{Effect on feedforward compensation}

In this section, the effects of parasitic stiffness on the feedforward compensation of floor vibrations are analysed. For this analysis, it is assumed that the feedforward compensation law (2.36) from section 2.6.2 is applied for each leg separately, see also equation (3.4).

$$
F_{i}(s)=-k_{i} y_{i}^{\prime}(s)
$$

In words, for each leg, the feedforward controller only generates anti-forces in the principal direction and these anti-forces depend only on the motion measured in the principal direction of the leg. It should be recognized that this results in a decoupled feedforward compensation in each of the principal directions. 


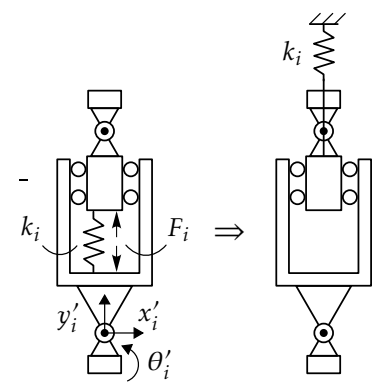

Figure 3.5: Augmented leg model (right) that is used to analyse the (ideal) feedforward compensation (3.4). The original leg model is shown on the left.

As a result, the transfer of vibration energy to the machine through the parasitic directions of the supports can not be prevented by the feedforward compensation. The achievable vibration isolation performance is therefore limited by the stiffness in the parasitic directions.

An elegant method to evaluate this performance limitation is by augmenting the leg model, as illustrated in figure 3.5. The original legs (shown on the left) are replaced by the model as shown on the right. In this augmented leg, the longitudinal stiffness of the original leg is set to zero. The leg is still rigid in bending and the hinges still have a non-zero torsional stiffness $c_{\theta}$. The principal support stiffness is now modelled by a skyhook spring, i.e. the restoring force only depends on the machine displacement.

As a result, the transfer of vibration energy from the support structure through the principal direction of the leg is eliminated, without altering the eigenfrequencies and mode shapes of the system. Therefore, this model correctly describes the system dynamics when ideal feedforward compensation is applied in the principal support directions.

The results of the singular value analysis for this system are also shown in figure 3.4. The dashed line shows the result for $c_{\theta}=1 \mathrm{Nm} / \mathrm{rad}$. The dash-dotted line shows the result for $c_{\theta}=0.1 \mathrm{Nm} / \mathrm{rad}$. Moreover, the soft mount reference transmissibility is indicated by the dotted line, see also equation (3.1).

Clearly, for a parasitic stiffness $c_{\theta}=1 \mathrm{Nm} / \mathrm{rad}$, the lower limit of the transmissibility is much larger than the soft mount reference level in the frequency range from approximately 8 to $80 \mathrm{~Hz}$. When the parasitic stiffness is reduced to $0.1 \mathrm{Nm} / \mathrm{rad}$, the effect on the vibration isolation is reduced to an acceptable level. In this case, the parasitic stiffness in each leg is less than $0.1 \%$ of the principal stiffness. It is expected to be a very challenging task to design a support with such a stiffness ratio. 
However, the addition of a feedback control system that dampens the suspension modes would also reduce the transmission of vibrations through the parasitic paths (in the frequency range of the suspension modes). As this is the case for the considered hard mount concept, $c_{\theta}=1 \mathrm{Nm} / \mathrm{rad}$ may be an acceptable value for the parasitic stiffness. In this case, the ratio of (parasitic) transverse stiffness to the principal stiffness is (only) in the order of $1 \%$.

Then, a practical guideline for the mechanical design of the hard mount in terms of the principal stiffness $k_{\text {principal }}$ and the parasitic stiffness $k_{\text {parasitic }}$ may be given by equation 3.5 .

$$
\frac{k_{\text {principal }}}{k_{\text {parasitic }}} \gtrsim 100
$$

\section{Effect on feedback control}

In figure 3.6, the model is shown that is used to analyse the effect of parasitic stiffness on the feedback control system. This model is a modified version of the model discussed in section 2.6.1, see figure 2.6.

The model consists of a leg with principal stiffness $k_{1}$ and a force actuator $F_{a}$. An additional parasitic stiffness $k_{p}$ is modelled parallel to $k_{1}$. This parasitic stiffness originates from other legs or cables. A force sensor $F_{m}$ and an absolute motion sensor $y_{1}^{a}$ are incorporated that can be used for feedback control. The floor motion $x_{0}$ is assumed to be an independent input.

The equation of motion for this system is given in equation (3.6). Equation (3.7) expresses the measured force $F_{m}$ as a function of the actuator force $F_{a}$ and the machine and floor displacement.

$$
\begin{aligned}
& \left(m s^{2}+k_{1}+k_{p}\right) X_{1}(s)=F_{a}(s)+\left(k_{1}+k_{p}\right) X_{0}(s) \\
& F_{m}(s)=F_{a}(s)-k_{1}\left(X_{1}(s)-X_{0}(s)\right)
\end{aligned}
$$

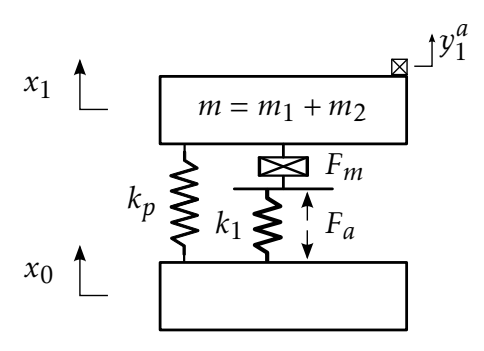

Figure 3.6: Model used for evaluating the effect of parasitic stiffness on feedback control 
Equations (3.8) and (3.9) present the transfer functions that are involved in the feedback loop for absolute motion feedback and force feedback, respectively. It is observed that the parasitic stiffness $k_{p}$ introduces a pair of transmission zeros in the force feedback transfer function $F_{m}(s) / F_{a}(s)$.

No additional transmission zeros occur when absolute motion feedback is used. The same is true for relative motion measurement. Therefore, the effects of parasitic stiffnesses on these feedback types are expected to be insignificant.

$$
\begin{aligned}
& \frac{X_{1}(s)}{F_{a}(s)}=\frac{1}{m s^{2}+k_{1}+k_{p}} \\
& \frac{F_{m}(s)}{F_{a}(s)}=\frac{m s^{2}+k_{p}}{m s^{2}+k_{1}+k_{p}}
\end{aligned}
$$

The transmission zeros in the force feedback transfer function can be troublesome if any of the zeros is unstable. It is well known that unstable zeros must lie well outside the control bandwidth to avoid deterioration of the feedback control performance, see e.g. [Skogestad and Postlethwaite (2005), Sect. 6.6]. Even when the transmission zeros are stable, the feedback design is greatly simplified when these zeros lie outside the control bandwidth. When the transmission zeros occur within the control bandwidth, additional $0 \mathrm{~dB}$-crossings are introduced in the loop gain, which make the controller tuning more difficult.

Either way, the frequency of any transmission zero (due to parasitic stiffness) should be sufficiently small, i.e. the parasitic stiffness should be small enough.

To derive a guideline for the stiffness ratio $k_{1} / k_{p}$, the following assumptions are made:

- The transmission zeros must lie at least a factor three below the lowest $0 \mathrm{~dB}$-crossing frequency.

- This lowest $0 \mathrm{~dB}$-crossing lies approximately a factor 10 below the open loop suspension frequency.

In this case, equation (3.10) gives a lower bound for the required ratio of the principal stiffness to the parasitic stiffness. Note that this bound is larger than the bound (3.5) and is therefore more difficult to realize in practice. This is an indication that the use of force sensors in the feedback loop is not advisable (at least not if the feedback control bandwidth is intended to extend over a relatively large frequency range).

$$
\frac{k_{\text {principal }}}{k_{\text {parasitic }}}=\frac{k_{1}}{k_{p}} \gtrsim 900
$$




\subsection{Recapitulation}

The active hard mount concept that is the research topic of this thesis is based on an exact-constraint mechanical design approach to realize sufficiently high support stiffness. A modular mount design is proposed, with six active legs, which incorporate force actuators and the necessary sensors.

The chosen control strategy is a combination of feedback control, which is intended to dampen the suspension modes and relevant structural modes, and a feedforward compensation of floor vibrations. The feedback and feedforward control designs are discussed in chapters 4 and 5, respectively.

From an analysis of a theoretical planar model it is found that the suspension frequencies should be grouped relatively close together. Otherwise, the open loop transmission of floor vibrations extents to unnecessarily high frequencies, which makes the control problem more challenging.

The effects of non-ideal constraints on the system dynamics have been evaluated using the singular value decomposition (SVD) of the (MIMO) transmissibility. When feedforward compensation of the floor vibration is applied, the performance limit due to the non-ideal constraints can be analysed through the SVD of an augmented system, where the principal support stiffness elements are replaced by skyhook springs.

From the analysis of a planar model, a rule of thumb is formulated, stating that the ratio of principal stiffness to parasitic stiffness should be at least 100 , in order to prevent the parasitic stiffness from deteriorating the vibration isolation performance. This rule of thumb is valid in case the feedback control uses absolute or relative motion measurements.

In case of force feedback control, the parasitic stiffness introduces additional transmission zeros. In this case, the stiffness ratio must be at least 900, in order to keep these transmission zeros outside the controller bandwidth. 



\section{Chapter 4}

\section{Active damping by feedback control}

In section 2.6.1, feedback control laws were presented which can add skyhook damping to the suspension mode, and simultaneously reduce the suspension mode frequency. Among these were PI-control using force measurement, PIcontrol using absolute acceleration measurement and PD-control using absolute velocity measurement.

However, the flexibility of the machine was not taken into account in the model for which the feedback laws were derived. In this chapter, this flexibility is taken into account and its effects on the achievable performance are evaluated. In section 4.2 it is discussed that the a single feedback control system can not achieve all the goals set forth in the previous chapter. In this thesis, the feedback control system is therefore extended with a feedforward controller that compensates for floor vibrations. The feedback control system is then designed for improving the suspension mode damping and the (relevant) structural mode damping.

The suitability of relative motion measurement, absolute motion measurement and force measurement for adding active structural damping is discussed in section 4.3. Moreover, their robustness properties are discussed. This discussion is based on Direct Velocity Feedback (DVF) control, which aims specifically at adding damping. 


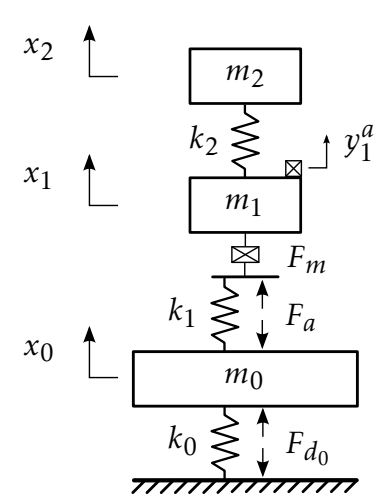

Figure 4.1: Extended model for active vibration isolation by feedback control, see table 2.1 for parameter values

\subsection{System model}

To evaluate the effects of floor dynamics and machine dynamics, the model of section 2.4 is used, which is repeated in figure 4.1. In this model, the flexibility of the floor and the machine are included. In order to focus on the effects of feedback control, any structural damping has been neglected.

In this chapter, feedback control based on three different measurement types is discussed, i.e. absolute motion, relative motion and force measurement. The transfer functions that are involved in the feedback loop are given by equations (4.1), (4.2) and (4.3), respectively. The system's characteristic polynomial $p(s)$ is expressed in equation (4.4). The transfer functions are readily derived from the model equations, which are presented in appendix A.1.2.

$$
\begin{aligned}
& \frac{X_{1}(s)}{F_{a}(s)}=\frac{\left(m_{0} s^{2}+k_{0}\right)\left(m_{2} s^{2}+k_{2}\right)}{p(s)} \\
& \frac{\Delta X(s)}{F_{a}(s)}=\frac{X_{1}(s)-X_{0}(s)}{F_{a}(s)}=\frac{\left(\left(m_{0}+m_{1}\right) s^{2}+k_{0}+k_{2}\right)\left(m_{2} s^{2}+k_{2}\right)-k_{2}^{2}}{p(s)} \\
& \frac{F_{m}(s)}{F_{a}(s)}=\frac{s^{2}\left(m_{0} s^{2}+k_{0}\right)\left(m_{1} m_{2} s^{2}+k_{2}\left(m_{1}+m_{2}\right)\right)}{p(s)} \\
& p(s)=\left(m_{0} s^{2}+k_{0}+k_{1}\right)\left(m_{1} s^{2}+k_{1}+k_{2}\right)\left(m_{2} s^{2}+k_{2}\right) \ldots \\
&-k_{2}^{2}\left(m_{0} s^{2}+k_{0}+k_{1}\right)-k_{1}^{2}\left(m_{2} s^{2}+k_{2}\right)
\end{aligned}
$$


It is important to note that the numerators are not the same for the respective measurement outputs. This difference is crucial in terms of the achievable structural damping, as will be discussed in section 4.3. Moreover, the difference in the zero dynamics also influences the stability robustness, which is discussed in more detail in section 4.3.1.

\subsection{Limitations of a 1 DOF controller}

In section 3.2.1, it has been stated that a single feedback controller can not simultaneously meet the three requirements of the active control system in a hard mount vibration isolation system, i.e. reduction of the floor vibration transmissibility and significant damping of the suspension mode(s) and relevant structural modes. Such a single controller is commonly referred to as a one degree-of-freedom (1 DOF) controller.

In this section, the reason behind this statement is presented. The discussion is focussed on feedback control based on absolute acceleration measurement, but the results can easily be extended to feedback control based on the other measurement types.

In section 2.6.1, it is shown that a combination of absolute acceleration and absolute velocity feedback can be used to reduce the suspension frequency and increase the damping of the suspension mode. In terms of absolute acceleration measurement, this constitutes a PI control law, as indicated in equation (4.5). The resulting loop gain $L(s)$ of the PI controller and the system dynamics is expressed in equation (4.6).

$$
\begin{aligned}
& K(s)=k_{a}+\frac{k_{v}}{s} \\
& L(s)=K(s) \frac{s^{2} X_{1}(s)}{F_{a}(s)}=\frac{s\left(k_{a} s+k_{v}\right)\left(m_{0} s^{2}+k_{0}\right)\left(m_{2} s^{2}+k_{2}\right)}{p(s)}
\end{aligned}
$$

Based on the rigid machine model of section 2.6.1, expressions relating the feedback parameters $k_{a}$ and $k_{v}$ to the desired suspension frequency $\omega_{\text {susp,des }}$ and the desired suspension damping $\zeta_{\text {susp,des }}$ can be derived, as shown in equations (4.7) and (4.8). In these equations, $\omega_{\text {susp }}$ is the open loop suspension frequency expressed in $\mathrm{rad} / \mathrm{s}$.

$$
\begin{aligned}
& k_{a}=\left(m_{1}+m_{2}\right)\left(\frac{\omega_{\text {susp }}^{2}}{\omega_{\text {susp,des }}^{2}}-1\right) \\
& k_{v}=2 \zeta_{\text {susp,des }} \omega_{\text {susp }}\left(m_{1}+m_{2}\right) \frac{\omega_{\text {susp }}}{\omega_{\text {susp,des }}}
\end{aligned}
$$




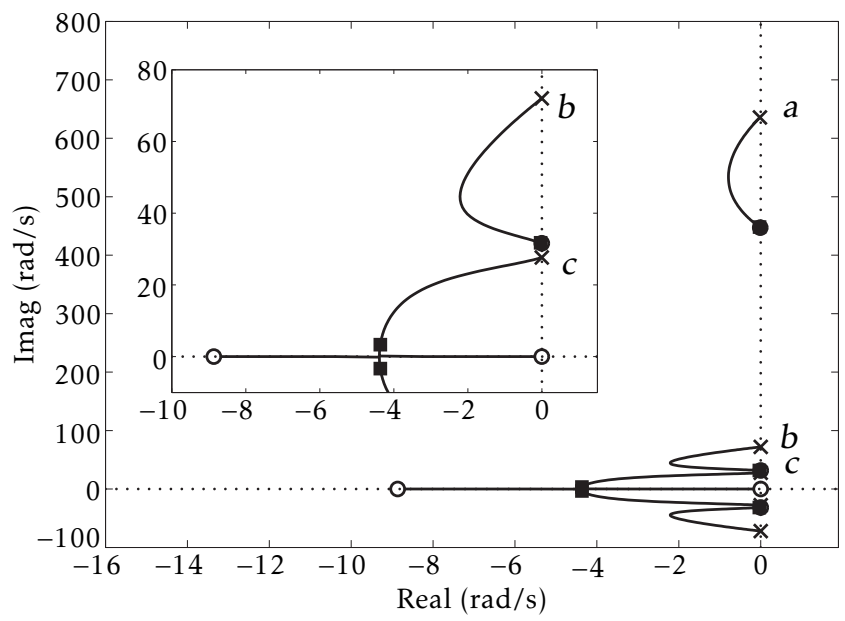

Figure 4.2: Root locus plot of $L(s)$, see equation (4.6); a: structural mode $(101 \mathrm{~Hz}), b$ : suspension mode $(11.5 \mathrm{~Hz}), \mathrm{c}$ : floor mode $(4.40 \mathrm{~Hz})$

$\times$ : open loop poles; $\quad$ п: closed loop poles; $\quad$ o: zeros

To investigate the performance of this PI controller in combination with the extended model of figure 4.1, the root locus plot of the loop gain $L(s)$ is considered, which is shown in figure 4.2. The open loop structural mode, suspension mode and floor mode resonances are marked by $a, b$ and $c$ respectively. The resulting closed loop poles are indicated by squares. The inset shows a close-up of the region near the origin.

The feedback parameters are computed from equations (4.7) and (4.8), with the desired suspension frequency taken as $1 \mathrm{~Hz}$ and $70 \%$ closed loop suspension damping. Using these parameters, the actual suspension frequency is $0.88 \mathrm{~Hz}$ and the suspension damping ratio is 0.79 . These values are reasonably close to the desired values.

However, it is observed in the root locus plot that the structural mode (a) and the floor mode (c) have moved to the open loop zeros and are still nearly undamped. Moreover, the structural mode frequency is reduced to $71 \mathrm{~Hz}$. Clearly, PI acceleration feedback is not capable of achieving all three objectives simultaneously.

This can be understood from the following observations on the physical equivalents of the feedback control action:

- At high frequencies (well above $2 \zeta_{\text {susp,des }} \omega_{\text {susp,des }}$ ), the PI acceleration feedback reduces to proportional acceleration feedback, meaning that the controller adds virtual mass.

- To improve the damping of the structural mode, velocity feedback should dominate in the vicinity of the structural resonance frequency. 
Unless the separation between the suspension mode and the structural mode frequencies is very large, these requirements for the controller behaviour correspond to overlapping frequency regions. Due to these conflicting requirements, it is not possible for a single feedback controller to simultaneously achieve a significant reduction of the suspension frequency as well as add sufficient damping to the structural mode and suspension mode.

\subsubsection{DOF control configurations}

Some of the limitations of a 1 DOF controller can be removed by augmenting the control system with additional sensors (and possibly actuators). Figure 4.3 illustrates three variations of such augmented control systems. For ease of reference, figure 4 .3a shows the 1 DOF control configuration.

One possibility is to add a control system $K_{2}(s)$ which specifically targets the problem of structural damping, see figure $4.3 \mathrm{~b}$. Such a system has been reported by e.g. [Holterman (2002)]. However, this requires additional hardware components that have to be incorporated into the machine itself. This may not always be possible. Another option is to use the cascaded feedback control system, as shown in figure $4.3 \mathrm{c}$. Once more, this requires modification of the machine.

In this thesis, the alternate cascaded configuration of figure $4.3 \mathrm{~d}$ is adopted. Here, the control system uses the measured machine motion and floor motion to improve the vibration isolation performance. The machine motion is used in a feedback controller $K_{1}(s)$ which is designed to improve the damping of the suspension and structural modes.

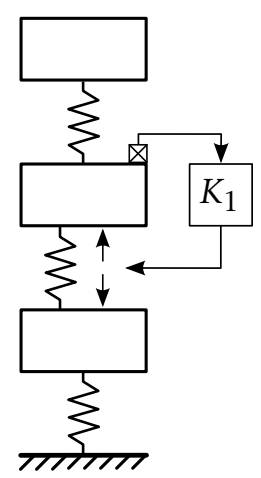

(a) $1 \mathrm{DOF}$

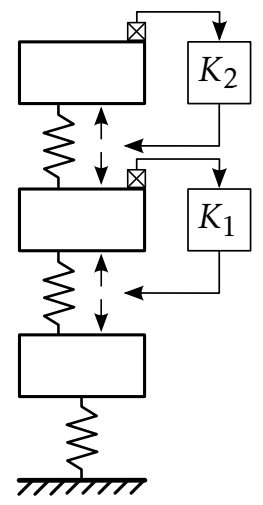

(b) $2 \times 1$ DOF

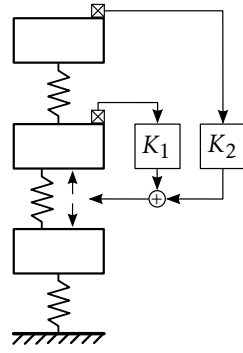

(c) cascaded 1

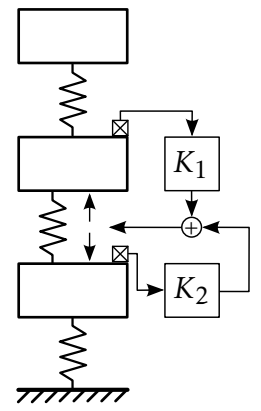

(d) cascaded 2

Figure 4.3: Various 1 DOF and 2 DOF control system configurations 
Moreover, floor vibration feedforward compensation $K_{2}(s)$ is added to reduce the transmissibility of the system. The advantage of this configuration is its modularity, i.e. all active components can be incorporated into the mount. In the remainder of this chapter, discuss methods for designing the feedback controller $K_{1}(s)$ are discussed. The feedforward compensation is discussed in detail in chapter 5 .

\subsection{DVF(-like) control}

In section 2.6.1 three feedback strategies that allow adding active suspension damping have been discussed, see equations (4.9)-(4.11). The first of these feedback laws is called Direct Velocity Feedback (DVF). As the name implies, DVF control employs proportional feedback of the measured velocity in order to add damping. The second feedback law is called Integral Force Feedback (IFF) for obvious reasons. The third feedback law uses proportional feedback of an absolute velocity signal. For all these feedback laws, negative feedback is used and the actual feedback controller $K_{1}(s)$ is purely a velocity feedback gain $k_{v}$.

Note that, in theory, DVF control is equivalent to derivative displacement feedback. Similarly, the proportional absolute velocity feedback is equivalent to integral acceleration feedback. ${ }^{1}$

- proportional feedback of a relative velocity measurement (DVF):

$$
F_{a}(s)=-k_{v} \Delta \dot{X}(s)=-k_{v} s \Delta X(s)
$$

- integral force feedback (IFF):

$$
F_{a}(s)=-k_{v} \frac{F_{m}(s)}{s}
$$

- proportional feedback of an absolute velocity measurement:

$$
F_{a}(s)=-k_{v} \dot{X}_{1}(s)=-k_{v} s X_{1}(s)
$$

All these feedback laws are capable of actively increasing the damping of the suspension mode. In this section, these control strategies are evaluated for their capability of adding active damping to the structural mode.

1 Bandwidth limitations of the sensors and controllers are ignored for now. 
The DVF control law is discussed first, based on the results from [Preumont (2002); Vervoordeldonk et al. (2006)]. These results are reproduced in appendix B.

For DVF control, the loop gain $L(s)$ (the product of the feedback controller and the system response) is given by equation (4.12), where the system response $\Delta X(s) / F_{a}(s)$ is expressed by equation (4.2).

By using modal expansion, the frequency response of the loop gain $L(s)$ in the vicinity of the structural resonance frequency $\omega_{r}$ can be approximated in many cases by either equation (4.13) or equation (4.14), depending on the location of the (closest) anti-resonance frequency $\omega_{a}$ with respect to the structural resonance frequency. Here, $\bar{k}_{v 1}$ and $\bar{k}_{v 2}$ are the effective feedback gains, see appendix B.1.

$$
\begin{aligned}
& L(s)=k_{v} \frac{s \Delta X(s)}{F_{a}(s)} \\
& L(s) \approx \frac{\bar{k}_{v 1}}{s} \frac{s^{2}+\omega_{a}^{2}}{s^{2}+\omega_{r}^{2}} \quad \omega_{a}<\omega_{r} \\
& L(s) \approx \bar{k}_{v 2} s \frac{s^{2}+\omega_{a}^{2}}{s^{2}+\omega_{r}^{2}} \quad \omega_{a}>\omega_{r}
\end{aligned}
$$

The maximum achievable damping $\zeta_{\max }$ for both cases is expressed by equation (4.15). The expressions for the optimal gain settings are more complicated and can be found in appendix B.2.

$$
\zeta_{\max }= \begin{cases}\frac{1}{2}\left(\frac{\omega_{r}}{\omega_{a}}-1\right) & \omega_{a}<\omega_{r} \\ \frac{1}{2}\left(\frac{\omega_{a}}{\omega_{r}}-1\right) & \omega_{a}>\omega_{r}\end{cases}
$$

The important conclusion from equation (4.15) is that the maximum achievable damping by DVF control is determined by the ratio of the resonance frequency and the anti-resonance frequency. Moreover, it appears that overcritical damping $\left(\zeta_{\max }>1\right)$ can be achieved for sufficiently large spacing between the anti-resonance and the structural resonance frequency. However, it is unlikely that such large frequency spacing is achieved in practice.

Interestingly, according to [Vervoordeldonk et al. (2006)], the structural damping may be improved further by using additional dynamic filters, i.e. by deviating from the pure DVF control law (4.9). 


\section{Comparison of the feedback control strategies}

In order to compare the capabilities of relative velocity measurement (DVF), force measurement (IFF) and absolute velocity measurement to add active structural damping, it is assumed that the loop gains in case of integral force feedback and absolute velocity feedback have the same form as either equation (4.13) or (4.14). In section 4.3.1, the validity of this assumption is discussed.

Then, the comparison comes down to comparing the anti-resonance frequencies $\omega_{a}$ for each feedback type, see equations (4.16)-(4.18). The expressions for the anti-resonance frequencies are obtained from the respective system responses (4.1)-(4.3), under the assumption that the anti-resonances that are associated with the floor resonance ${ }^{2}$ occur at a much lower frequency (which is typically the case in practice).

$$
\begin{aligned}
& \omega_{a}^{\text {force }}=\sqrt{\frac{k_{2}}{m_{2}}\left(1+\frac{m_{2}}{m_{1}}\right)} \\
& \omega_{a}^{\mathrm{abs}}=\sqrt{\frac{k_{2}}{m_{2}}} \\
& \omega_{a}^{\mathrm{rel}} \gtrsim \sqrt{\frac{k_{2}}{m_{2}}\left(1+\frac{m_{2}}{m_{0}+m_{1}}\right)}
\end{aligned}
$$

For typical vibration isolation problems, the structural resonance frequency $\omega_{r}$ is the largest resonance frequency of the model shown in figure 4.1. By disregarding the floor stiffness $k_{0}$ and the suspension stiffness $k_{1}$, an approximation of the structural resonance frequency $\omega_{r}$ is found, see equation (4.19).

$$
\omega_{r} \gtrsim \sqrt{\frac{k_{2}}{m_{2}}\left(1+\frac{m_{2}}{m_{1}}\right)}
$$

By comparing equation (4.19) and (4.16), it is concluded that in case of force feedback a pole/zero cancellation occurs for the structural mode, i.e. the ratio of $\omega_{r}$ to $\omega_{a}$ is (nearly) one. From equation (4.15), it is concluded that it is impossible to significantly improve the damping of the structural mode by force feedback.

In the case of absolute and relative velocity feedback, the spacing between the resonance and anti-resonance frequency depends on the mass ratios $m_{2} / m_{1}$ and $m_{2} /\left(m_{0}+m_{1}\right)$ respectively. If these ratios are sufficiently large, absolute

2 These anti-resonances mostly depend on the model parameters $m_{0}$ and $k_{0}$. 
and relative velocity feedback can be used to improve the structural damping. However, recall from section 2.6.1 that relative velocity feedback does not provide skyhook damping for the suspension mode, i.e. the high-frequency roll-off in the transmissibility is $-20 \mathrm{~dB} /$ decade instead of the desired -40 $\mathrm{dB} /$ decade. Therefore, it can be concluded that absolute velocity feedback control results in the best overall vibration isolation performance.

The dependency of the achievable damping on the mass ratios $m_{2} / m_{1}$ and $m_{2} /\left(m_{0}+m_{1}\right)$ for the system model of section 4.1 can be generalized to more realistic machines. Then, it is concluded that the mass distribution in the machine greatly influences the achievable damping by feedback control. The mass distribution can be influenced by the machine design as well as the choice for the locations of the supports.

\subsubsection{Stability robustness of DVF-like control}

In this section, the stability robustness properties of the proposed control strategies are investigated. For this purpose, the set of linear ordinary differential equations, as shown in equation (4.20) is used. This system can be used to model the (small) vibrations of a general flexible structure.

$$
\boldsymbol{M} \ddot{\boldsymbol{q}}(t)+\boldsymbol{D} \dot{\boldsymbol{q}}(t)+\boldsymbol{K} \boldsymbol{q}(t)=\boldsymbol{B}_{d} \boldsymbol{d}(t)+\boldsymbol{B}_{u} \boldsymbol{u}(t) \quad \boldsymbol{q} \in \mathbb{R}^{N_{q}}, \boldsymbol{u} \in \mathbb{R}^{N_{u}}, \boldsymbol{d} \in \mathbb{R}^{N_{d}}
$$

Here, $\boldsymbol{q}$ is the vector of generalized coordinates and $\boldsymbol{d}$ and $\boldsymbol{u}$ are the (generalized) disturbances and actuator signals. $\boldsymbol{B}_{d}$ and $\boldsymbol{B}_{u}$ represent the interaction of the disturbances and actuators with the flexible structure. $\boldsymbol{M}, \boldsymbol{D}$ and $\boldsymbol{K}$ are the (generalized) mass, damping and stiffness matrices.

$\boldsymbol{M}$ and $\boldsymbol{K}$ are assumed to be positive definite ${ }^{3}(>0), \boldsymbol{D}$ is assumed to be positive (semi-)definite $(\geq 0)$. As a result, the open loop system is (marginally) stable.

DVF control is considered first, which is known to possess excellent stability robustness properties in case the velocity sensor and the force actuator are collocated [Balas (1979); Joshi (1985)]. In that case, the sensor and actuator form a dual pair, meaning that the inner product of the sensor and actuator signals represents the total power supplied to the system by the actuator [Holterman et al. (2002); Preumont (2002)].

3 A matrix $\boldsymbol{A} \in \mathbb{R}^{n \times n}$ is positive (semi-)definite when $\boldsymbol{x}^{\mathrm{T}} \boldsymbol{A} \boldsymbol{x}>(\geq) 0, \forall x \in \mathbb{R}^{n}, \boldsymbol{x} \neq 0$ This is commonly denoted by $\boldsymbol{A}>(\geq) 0$ 
The favourable stability robustness of DVF control can also be deduced from the model of equation (4.20). The collocated velocity measurement is expressed by equation (4.21). By applying the DVF feedback law of equation (4.22), the closed loop system dynamics are expressed by equation (4.23).

$$
\begin{aligned}
& y(t)=\boldsymbol{B}_{u}^{\mathrm{T}} \dot{\boldsymbol{q}}(t) \\
& \boldsymbol{u}(t)=-\boldsymbol{G} y(t) \quad \boldsymbol{G}>0 \\
& \boldsymbol{M} \ddot{\boldsymbol{q}}(t)+\left[\boldsymbol{D}+\boldsymbol{B}_{u} \boldsymbol{G} \boldsymbol{B}_{u}^{\mathrm{T}}\right] \dot{\boldsymbol{q}}(t)+\boldsymbol{K} \boldsymbol{q}(t)=\boldsymbol{B}_{d} \boldsymbol{d}(t)
\end{aligned}
$$

The closed-loop system is stable when the damping matrix $\left[\boldsymbol{D}+\boldsymbol{B}_{u} \boldsymbol{G} \boldsymbol{B}_{u}^{\mathrm{T}}\right]$ is positive definite. $\boldsymbol{D}$ is positive (semi-)definite by assumption, i.e. the openloop system is not unstable.

The term $\boldsymbol{B}_{u} \boldsymbol{G} \boldsymbol{B}_{u}^{\mathrm{T}}$ is a quadratic matrix product and is therefore at least positive semi-definite. Because the feedback gain matrix $\boldsymbol{G}$ is positive definite, the term $\boldsymbol{B}_{u} \boldsymbol{G} \boldsymbol{B}_{u}^{\mathrm{T}}$ is only zero for any $\dot{\boldsymbol{q}}$ in the zero dynamics of the system. These zero dynamics are the dynamics for which the measurement is identically zero, i.e. the null space or kernel of $\boldsymbol{B}_{u}^{\mathrm{T}}$ as indicated in equation (4.24).

As a result, when the zero dynamics are stable (see condition (4.25)), the closed-loop damping matrix is positive definite and the closed-loop system is asymptotically stable.

$$
\begin{aligned}
& \operatorname{ker}\left(\boldsymbol{B}_{u}^{\mathrm{T}}\right)=\left\{\dot{\boldsymbol{q}} \in \mathbb{R}^{N_{q}} \mid \boldsymbol{B}_{u}^{\mathrm{T}} \dot{\boldsymbol{q}}=0\right\} \\
& \dot{\boldsymbol{q}}^{\mathrm{T}} \boldsymbol{D} \dot{\boldsymbol{q}}>0 \quad \forall \dot{\boldsymbol{q}} \in \operatorname{ker}\left(\boldsymbol{B}_{u}^{\mathrm{T}}\right)
\end{aligned}
$$

The robustness of this closed-loop system stems from the fact that the stability depends only on the collocation of the sensors and actuators, the positive definiteness of the feedback gain matrix $G$ and the stability of the zero dynamics. It does not depend on the system's mass and stiffness parameters. Moreover, robust stability is maintained when small collocation errors occur and in the presence of certain sensor dynamics and/or non-linearities [Joshi (1985)].

Clearly, the DVF approach can be easily used in MIMO systems, provided that the feedback gain matrix $G$ is chosen as a positive definite matrix. However, the (optimal) tuning of the controller parameters becomes more involved [Holterman et al. (2002)]. 


\section{Interpretation in terms of passivity}

Actually, DVF control is an example of passivity-based control, see e.g. [Ortega et al. (1998); van der Schaft (2000); Bao and Lee (2007)]. This is discussed in greater detail in appendix B.3, where it is discussed that the collocated velocity measurement results in a passive open loop system. Some relevant properties of (linear) passive systems are listed below:

- if the system is detectable ${ }^{4}$, any static gain negative output feedback asymptotically stabilizes the system

- the system's transfer function $\boldsymbol{H}(s)$ is minimum phase

- the transfer function has bounded phase: for a SISO system, $\angle H(j \omega) \in\left[-90^{\circ}, 90^{\circ}\right]$

The first property is nothing more than a restatement of the previously obtained result. Note that the detectability requirement is satisfied when condition (4.25) is satisfied.

The latter property of phase boundedness may proof useful when the collocation condition is violated, which is investigated in the following subsections. It should be pointed out that, for (poorly damped) mechanical structures, the bounded phase property means that the transfer function must have alternating poles and zeros along/near the imaginary axis. This is also put forward by [Preumont (2002)].

\section{Collocation of force feedback}

In [Preumont et al. (2002)], it is argued that collocated force measurement also results in an alternating pole/zero pattern. Hence, even though the force sensor and force actuator do not form a dual pair, integral force feedback possesses similar stability robustness properties as DVF. This also means that the loop gain approximations of equations (4.13) and (4.14) are valid.

\section{Collocation of absolute motion feedback}

The situation is quite different in case of absolute velocity measurement. Although the inner product of the measurement and the actuator signal represents a power signal, it does not equal the total power supplied to the system by the actuator.

4 i.e. all unstable modes are observable 
As an example, equation (4.26) expresses the equations of motion for the model of section 4.1 . The absolute velocity measurement is expressed in equation (4.27), from which it is immediately clear that the velocity measurement is not collocated with the force actuation.

$$
\begin{aligned}
& \underbrace{\left[\begin{array}{ccc}
m_{0} & 0 & 0 \\
0 & m_{1} & 0 \\
0 & 0 & m_{2}
\end{array}\right]}_{\boldsymbol{M}} \underbrace{\left[\begin{array}{c}
\ddot{x}_{0} \\
\ddot{x}_{1} \\
\ddot{x}_{2}
\end{array}\right]}_{\ddot{\boldsymbol{q}}}+\underbrace{\left[\begin{array}{ccc}
k_{0}+k_{1} & -k_{1} & 0 \\
-k_{1} & k_{1}+k_{2} & -k_{2} \\
0 & -k_{2} & k_{2}
\end{array}\right]}_{\boldsymbol{K}} \underbrace{\left[\begin{array}{c}
x_{0} \\
x_{1} \\
x_{2}
\end{array}\right]}_{\boldsymbol{q}}=\underbrace{\left[\begin{array}{c}
-1 \\
1 \\
0
\end{array}\right]}_{\boldsymbol{B}_{u}} F_{a} \\
& y=\dot{x}_{1}=\left[\begin{array}{lll}
0 & 1 & 0
\end{array}\right] \dot{\boldsymbol{q}} \neq \boldsymbol{B}_{u}^{\mathrm{T}} \dot{\boldsymbol{q}}
\end{aligned}
$$

However, in section 4.2 feedback control based on (absolute) acceleration measurement has been discussed for this model. In the root locus plot of figure 4.2 an alternating pole/zero pattern is observed for this model. Moreover, it is clear from that root locus plot that any choice for the positive feedback parameters $k_{a}$ and $k_{v}$ stabilizes that particular system. Apparently, the model parameters used in section 4.2 result in a "practically collocated" system.

Therefore, it is interesting to determine a model parameter set for which the system of equations (4.26) and (4.27) is practically collocated, i.e. for which the poles and zeros alternate along the imaginary axis. Note that the limiting case for alternating poles and zeros is a pole-zero cancellation. Therefore, a set of parameters is derived which result in an exact pole-zero cancellation first.

This particular system has two anti-resonances, which are expressed in equation (4.28) (see also equation (4.1)). The poles of the open-loop system are the roots of the characteristic polynomial, which is expressed in equation (4.29). The expansion of this expression was presented in equation (4.4).

$$
\begin{array}{ll}
\omega_{a, 0}=\sqrt{\frac{k_{0}}{m_{0}}} \quad \omega_{a, 2}=\sqrt{\frac{k_{2}}{m_{2}}} \\
p(s)=\operatorname{det}\left(\boldsymbol{M} s^{2}+\boldsymbol{K}\right) \quad s \in \mathbb{C}
\end{array}
$$

By setting $s=j \omega_{a, 0}$ and $s=j \omega_{a, 2}$ in the characteristic polynomial, parameter sets can be computed for which a pole-zero cancellation occurs. These parameter sets are expressed in equation (4.30).

$$
\begin{aligned}
& s=j \omega_{a, 0} \quad \Leftrightarrow \quad \frac{k_{0}}{m_{0}}=\frac{k_{2}}{m_{2}}\left(1+\frac{m_{2}}{m_{1}}\right) \\
& s=j \omega_{a, 2} \quad \Leftrightarrow \quad \frac{k_{2}}{m_{2}}=\frac{k_{0}+k_{1}}{m_{0}}
\end{aligned}
$$


Finally, it can be checked that pole-zero flipping does not occur, i.e. the system is practically collocated, when either of the conditions in equation (4.31) is satisfied.

$$
\sqrt{\frac{k_{2}}{m_{2}}\left(1+\frac{m_{2}}{m_{1}}\right)}<\sqrt{\frac{k_{0}}{m_{0}}} \quad \vee \quad \sqrt{\frac{k_{2}}{m_{2}}}>\sqrt{\frac{k_{0}+k_{1}}{m_{0}}}
$$

For vibration isolation systems, the first condition amounts to a test on the structural resonance mode (compare equation (4.19)). It states that the structural resonance should be smaller than the resonance frequency of the floor (without the machine). It is highly unlikely that this condition is met for practical machines, as the first resonance frequencies of floors typically occur near $10 \mathrm{~Hz}$, see e.g. [Howard and Hansen (2003)].

The second condition is a test on the anti-resonance frequency in the system response (see equation (4.17)). Assuming that the support stiffness $k_{1}$ is smaller than the floor stiffness $k_{0}$, this condition is always satisfied when the anti-resonance frequency is at least a factor $\sqrt{2}$ larger than the floor resonance frequency (without the machine). It is expected that this is the case for most vibration isolations systems, and therefore absolute motion measurement will result in a practically collocated system.

\section{Concluding remarks}

The presented robustness analysis assumed perfect sensors and actuators, e.g. infinite bandwidth and no delay. In practice, these sensor and actuator limitations deteriorate the stability robustness. However, when the effects are minor within the desired control bandwidth, the resulting stability margins will remain large.

It was mentioned previously that passive systems (and therefore collocated systems as well) are minimum phase, meaning that all poles and zeros are in the left half-plane (LHP) in the continuous case, or inside the unit circle for discrete systems. However, it is well known that in sampled-data systems non-minimum phase zeros (NMP) can occur due to sampling [Åström et al. (1984)]. Hence, when a discrete control system is used, the occurrence of NMP zeros should be checked.

Moreover, when the sensor can not be located at the same location due to spatial limitations, NMP zeros may occur. 


\subsection{Recapitulation}

In this chapter, feedback control strategies have been examined that are able to add active damping to the suspension and (relevant) structural modes. In section 4.2 it was shown that adding active damping to the these modes can not be combined with the (active) reduction of the suspension resonance frequency, without adding an additional degree-of-freedom to the control system. In this thesis, a feedback controller is used to add active damping to the suspension and structural modes and feedforward compensation of floor vibrations is used to reduce the transmissibility of floor vibrations. This feedforward compensation is the subject of chapter 5 .

It has been shown that the ratio of the structural resonance frequency to its associated anti-resonance frequency is the key factor in determining the achievable active damping in the structural resonance mode. Based on this result, absolute motion measurement (velocity or acceleration) is found to be best suited for adding active damping to both the suspension and structural modes. When a force measurement is used for feedback control, it is impossible to add damping to the structural mode due to a pole-zero cancellation. Relative motion feedback (DVF) is capable of improving the structural damping, but can not achieve skyhook damping of the suspension mode.

The excellent stability robustness properties of DVF control have been derived from a general model for a flexible structure. The relationship to passivitybased control is discussed briefly. Although absolute motion measurement does not necessarily provide such excellent stability robustness, conditions for which the system is practically collocated have been derived. It is expected that these conditions are easily satisfied in practice. 


\section{Chapter 5}

\section{Feedforward compensation of floor vibrations}

This chapter discusses the feedforward compensation approach that is used to improve the response to floor vibrations. In section 5.1 the system model that is used in this chapter is introduced. Then, the optimal controller and resulting performance are discussed in section 5.2.

However, for reasons discussed in section 5.2.5, an adaptive feedforward control scheme is required to find the optimal controller. In section 5.3, the adaptive control approach is presented, which is based on the filtered-x LMS adaptive algorithm that is widely used in Active Noise Control (ANC) applications. A recapitulation of the chapter is presented in section 5.4.

\subsection{System model}

In this chapter, a generic system model is used to discuss the theory of (adaptive) feedforward control. A block diagram of this model is shown in figure 5.1. The model is expressed in the discrete-time domain, because the feedforward controller will be implemented digitally.

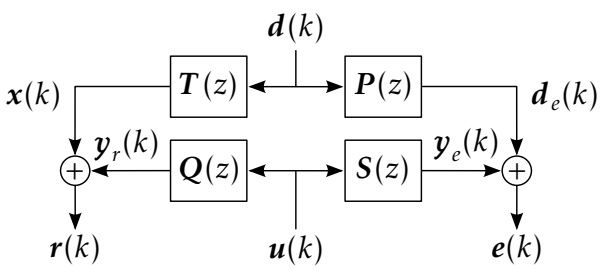

Figure 5.1: Block diagram of the generic system model used for discussion of the feedforward control theory. 
Loosely speaking, the signals in this model can be given the following interpretation within the framework of vibration isolation problems:

- $\boldsymbol{d}(k)$ : This disturbance signal represents the disturbance sources that cause floor vibrations. In this thesis, it is assumed that these disturbance sources are random, wide-sense stationary signals.

Note that the floor vibrations itself are not considered as disturbance sources, but rather as caused by the (true) disturbance sources.

- $\boldsymbol{u}(k)$ : This control signal represents the feedforward compensation signals, i.e. the generated anti-forces.

- $\boldsymbol{e}(k)$ : This error signal denotes the measured error signals that are available for optimization of the feedforward controller.

The total error signal is formed by two signal components: a component $\boldsymbol{d}_{e}(k)$ that is caused by the disturbance signal and a component $y_{e}(k)$ that is the result of the control action.

- $\boldsymbol{r}(k)$ : This reference signal denotes the measured floor vibrations that are used to compute the feedforward compensation signals. Like the error signal, it is formed by two components: a component $x(k)$ that is caused by the disturbance signal and a component $y_{r}(k)$ due to the control action. The latter component can be interpreted as the floor vibrations generated by the feedforward compensation forces (as these forces are also exerted on the floor).

The signals in the model are related through four transfer paths: the primary path $P(z)$, the secondary path $S(z)$, the tertiary path $T(z)$ and the quaternary path $Q(z)$, see equation (5.1).

$$
\left[\begin{array}{l}
E(z) \\
R(z)
\end{array}\right]=\left[\begin{array}{ll}
P(z) & S(z) \\
T(z) & Q(z)
\end{array}\right]\left[\begin{array}{l}
D(z) \\
U(z)
\end{array}\right]
$$

All these transfer paths are assumed to be stable, linear, time-invariant (LTI) systems. The stability assumption is reasonable for vibration isolation problems, where any unstable plant dynamics will be stabilized by a feedback control system. Whenever a feedback control system is present, the transfer paths are assumed to represent the closed loop dynamic behaviour.

Although the interpretation as presented above is helpful in gaining insight in the physical background of the problem, it must be stressed that the situation is actually more subtle. The output signals $\boldsymbol{e}(k)$ and $\boldsymbol{r}(k)$ actually represent the sampled and digitized versions of the measured vibrations. Likewise, the control signal $\boldsymbol{u}(k)$ is the digitized version of the generated anti-forces.

Therefore, besides describing the system mechanics, the transfer paths $P(z)$, $S(z), T(z)$ and $Q(z)$ must also describe the sensor and actuator dynamics, including anti-aliasing and reconstruction filters as well as the AD- and DAconverters. 


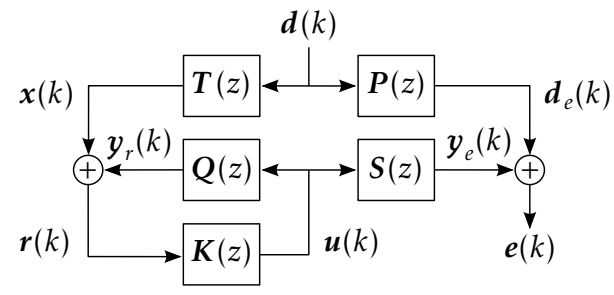

Figure 5.2: Block diagram of the system model including the "feedforward" controller $K(z)$.

\subsubsection{Internal model compensation}

In figure 5.2, the "feedforward" controller $K(z)$ has been included in the block diagram of the system model. Immediately, it is clear that the quaternary path $Q(z)$ and the controller $K(z)$ form a feedback loop. Physically, this is as expected because the control actuator generates forces that are also exerted in the floor, causing vibrations that are in turn measured by the reference sensor.

Therefore, referring to $\boldsymbol{K}(z)$ as a feedforward controller is incorrect. However, throughout this thesis internal model compensation (IMC) will be used to recast the control problem as a feedforward problem.

In figure 5.3, an internal model $\hat{Q}(z)$ of the quaternary path is used to eliminate the contribution $y_{r}(k)$ to the reference signal $\boldsymbol{r}(k)$. The overall controller $K(z)$ is indicated by the dashed box and is given by equation (5.2).

$$
\boldsymbol{K}(z)=\boldsymbol{W}(z)(\boldsymbol{I}+\hat{\mathbf{Q}}(z) \boldsymbol{W}(z))^{-1}
$$

Under the assumption that $Q(z)$ is stable and the internal model $\hat{Q}(z)$ is perfect, any stable controller $\boldsymbol{W}(z)$ internally stabilizes the feedback loop [Zames (1981)]. This parametrization of all internally stabilizing controllers is well known as the Youla, Youla-Kučera or Q-parametrization, see e.g. [Skogestad and Postlethwaite (2005), Sect. 4.8].

In the presence of model errors in $\hat{\mathbf{Q}}(z)$, a sufficient requirement for stability of the feedback loop follows from the small-gain theorem, as stated by equation (5.3). Here, $\Delta \boldsymbol{Q}\left(\mathrm{e}^{j \omega}\right)=\boldsymbol{Q}\left(\mathrm{e}^{j \omega}\right)-\hat{\mathbf{Q}}\left(\mathrm{e}^{j \omega}\right)$ represents the model error at the normalized frequency $\omega(\pi \leq \omega \leq \pi)$ and $\|\cdot\|_{\infty}$ denotes the $\mathcal{H}_{\infty}$-norm.

$$
\left\|\boldsymbol{W}\left(\mathrm{e}^{j \omega}\right) \Delta \boldsymbol{Q}\left(\mathrm{e}^{j \omega}\right)\right\|_{\infty}<1
$$




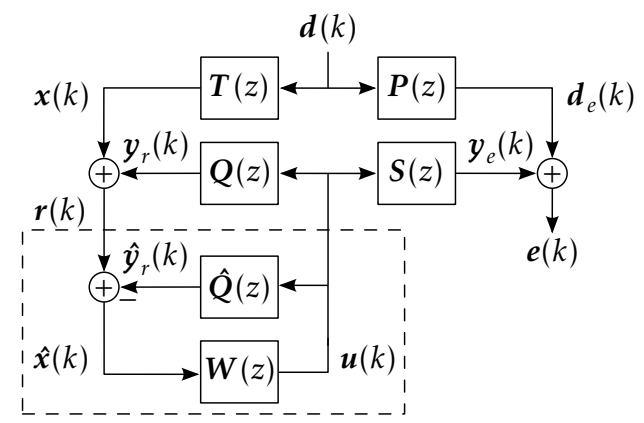

Figure 5.3: Block diagram showing the internal model compensation for $Q(z)$. The dashed box indicates the overall controller $K(z)$.

From the stability requirement (5.3), it is concluded that, in general, the model error $\Delta \boldsymbol{Q}\left(\mathrm{e}^{j \omega}\right)$ should be small at frequencies where the magnitude of $\boldsymbol{W}\left(\mathrm{e}^{j \omega}\right)$ is expected to be large. As a result, the quality of the model $\hat{Q}(z)$ should be very high at these frequencies. In section 7.3, a system identification method is discussed that can be used to obtain such accurate models for the quaternary path (and other transfer paths).

For the remainder of this chapter, it is assumed that the quaternary path is known exactly. In this case, the block diagram of figure 5.3 can be reduced to the diagram shown in figure 5.4, in which $W(z)$ is clearly a feedforward controller. The discussion of (optimal and adaptive) feedforward control theory in this chapter is based on this latter system model.

\subsection{Optimal feedforward control}

In this section, optimal feedforward control is discussed. In the following section, the optimization criterion is presented and the theoretical optimal solution is given. Then, in section 5.2.2, a constrained optimal controller is presented, which is constrained to be stable and causal. As a result, this controller can be implemented in real-time. Finally, in section 5.2.3, the effects of measurement noise on the Causal Wiener filter are discussed. The results in this section are mostly based on the work by [Fraanje (2004); Nijsse (2006)].

\subsubsection{Performance measure}

The objective of the feedforward controller is to minimize (in some sense) the error signal $\boldsymbol{e}(k)$. It is convenient to use the squared RMS-norm of the error signal (the Mean Squared Error (MSE)) for this purpose, see equation (5.4).

$$
J_{\mathrm{MSE}}(\boldsymbol{W})=\|\boldsymbol{e}(k)\|_{\mathrm{RMS}}^{2}
$$




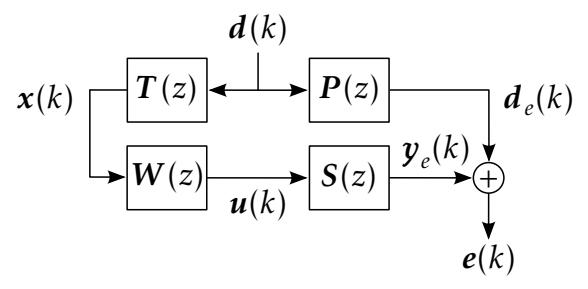

Figure 5.4: Equivalent feedforward control model of figure 5.3, assuming perfect knowledge of the quaternary path $Q(z)$.

Let $\boldsymbol{\Phi}_{\boldsymbol{d}}(z)$ denote the spectral factor of the disturbance signal $\boldsymbol{d}(k)$. Then, by using Parseval's theorem, the cost criterion $J_{\text {MSE }}$ can also be expressed by equation (5.5), where (.) is a shorthand notation for $\left(\mathrm{e}^{j \omega}\right)$.

$$
\begin{aligned}
J_{\mathrm{MSE}}(\boldsymbol{W}) & =\frac{1}{2 \pi} \int_{-\pi}^{\pi}\left\|\boldsymbol{P}(.) \boldsymbol{\Phi}_{\boldsymbol{d}}(.)+\boldsymbol{S}(.) \boldsymbol{W}(.) \boldsymbol{T}(.) \boldsymbol{\Phi}_{\boldsymbol{d}}(.)\right\|_{F}^{2} \mathrm{~d} \omega \\
& =\left\|\boldsymbol{P}(z) \boldsymbol{\Phi}_{\boldsymbol{d}}(z)+\boldsymbol{S}(z) \boldsymbol{W}(z) \boldsymbol{T}(z) \boldsymbol{\Phi}_{\boldsymbol{d}}(z)\right\|_{2}^{2}
\end{aligned}
$$

The optimal controller is then defined as the solution to the minimization problem (5.6).

$$
\boldsymbol{W}_{\mathrm{opt}}(z)=\arg \min _{\boldsymbol{W}} J_{\mathrm{MSE}}(\boldsymbol{W})
$$

From equation (5.5), it can be concluded that the performance measure is reduced to zero when the controller is chosen according to equation (5.7). Here, $S^{\ddagger}(z)$ is the right inverse of $S(z)$ and $T^{\dagger}(z)$ is the left inverse of $T(z)$.

$$
\boldsymbol{W}_{\text {opt }}(z)=-S^{\ddagger}(z) P(z) T^{\dagger}(z)
$$

However, this optimal controller $W_{\text {opt }}(z)$ can only be implemented in realtime, when it is causal. Moreover, $\boldsymbol{W}_{\text {opt }}(z)$ must be stable in order to internally stabilize the feedback loop, as discussed in section 5.1.1. This requires both $S(z)$ and $\boldsymbol{T}(z)$ to have causal and stable (right or left) inverses. In most practical problems, these conditions are not met due to delays and unstable (non-minimum phase) zeros in the secondary path $S(z)$ and the tertiary path $T(z)$. As a result, perfect cancellation can generally not be achieved.

Finally, it is pointed out that it is straightforward to include frequency weighing in the cost criterion, by multiplying $P(z)$ and $S(z)$ from the left by an appropriate dynamic filter. 


\subsubsection{Causal Wiener filter}

The solution to the optimization problem (5.6), with the added constraints of stability and causality, is commonly referred to as the Causal Wiener filter. In order to find the Causal Wiener filter, (co-)inner-outer factorizations of the secondary path $S(z)$ and the tertiary path $T(z)$ are used. These (co-)inner-outer factorizations are presented first.

\section{(Co-) inner-outer factorization}

The information in this section has been taken largely from [Ionescu and Oară (1996); Fraanje (2004)]. The interested reader is referred to these sources or e.g. the textbooks on system theory [Vidyasagar (1985); Chen et al. (2004)] for further details and algorithms.

Let $G(z)$ be a proper, stable transfer function with $N_{u}$ inputs and $N_{y}$ outputs and with normal rank $R \leq \min \left(N_{y}, N_{u}\right)^{1}$. Then, the inner-outer factorization of $G(z)$ is given by equation (5.8).

$$
\boldsymbol{G}(z)=\boldsymbol{G}_{i}(z) \boldsymbol{G}_{o}(z)
$$

The outer factor $G_{o}(z)$ is stable, proper and minimum-phase. It has $N_{u}$ inputs and $R$ outputs. Because it is minimum-phase and $R \leq N_{u}$, it has a stable right inverse.

The inner factor $\boldsymbol{G}_{i}(z)$ is stable, proper and all-pass. The inner factor has $R$ inputs and $N_{y}$ outputs. Moreover, when the inner factor is not square $\left(R<N_{y}\right)$, an orthogonal inner factor $G_{i}^{\perp}(z)$ exists with $N_{y}$ outputs and $N_{y}-R$ inputs, such that $\left[G_{i}(z) G_{i}^{\perp}(z)\right]$ is a unitary system ${ }^{2}$, see equations (5.9) and (5.10). Here, $G_{i}^{*}(z)$ denotes the adjoint system of $G_{i}(z)$ i.e. the transposed and timereversed system: $G_{i}^{*}(z) \equiv G_{i}^{\mathrm{T}}\left(z^{-1}\right)$.

$$
\begin{aligned}
{\left[\begin{array}{ll}
\boldsymbol{G}_{i}(z) & \boldsymbol{G}_{i}^{\perp}(z)
\end{array}\right]\left[\begin{array}{c}
\boldsymbol{G}_{i}^{*}(z) \\
\boldsymbol{G}_{i}^{\perp *}(z)
\end{array}\right] } & =\boldsymbol{G}_{i}(z) \boldsymbol{G}_{i}(z)^{*}+\boldsymbol{G}_{i}^{\perp}(z) \boldsymbol{G}_{i}^{\perp *}(z)=\boldsymbol{I}_{N_{y}} \\
{\left[\begin{array}{c}
\boldsymbol{G}_{i}^{*}(z) \\
\boldsymbol{G}_{i}^{\perp *}(z)
\end{array}\right]\left[\begin{array}{ll}
\boldsymbol{G}_{i}(z) & \left.\boldsymbol{G}_{i}^{\perp}(z)\right]
\end{array}\right] } & {\left[\begin{array}{cc}
\boldsymbol{G}_{i}^{*}(z) \boldsymbol{G}_{i}(z) & \boldsymbol{G}_{i}^{*}(z) \boldsymbol{G}_{i}^{\perp}(z) \\
\boldsymbol{G}_{i}^{\perp *}(z) \boldsymbol{G}_{i}(z) & \boldsymbol{G}_{i}^{\perp *}(z) \boldsymbol{G}_{i}^{\perp}(z)
\end{array}\right] } \\
& =\left[\begin{array}{cc}
\boldsymbol{I}_{R} & \mathbf{0} \\
\mathbf{0} & \boldsymbol{I}_{N_{y}-R}
\end{array}\right]=\boldsymbol{I}_{N_{y}}
\end{aligned}
$$

$1 R=\sup \{\operatorname{rank}(G(\lambda)), \lambda \in \mathbb{C}, \lambda$ not a pole of $G(z)\}$

2 A square system $\boldsymbol{G}(z)$ is unitary when $\boldsymbol{G}(z) \boldsymbol{G}(z)^{*}=\boldsymbol{G}(z)^{*} \boldsymbol{G}(z)=\boldsymbol{I}$. This is a generalization of the concept of unitary matrices to linear systems. 
The key idea behind the inner-outer factorization is that the outer factor contains all the system dynamics that can be stably and causally inverted. The inner factor contains the remaining dynamics (which are due to delays and non-minimum phase zeros). Note that the inner factor contains only phase information, because it is all-pass. As a corollary, $G_{o}(z)$ is a spectral factor of $\boldsymbol{G}^{*}(z) \boldsymbol{G}(z)$, i.e. $\boldsymbol{G}_{o}^{*}(z) \boldsymbol{G}_{o}(z)=\boldsymbol{G}^{*}(z) \boldsymbol{G}(z)$.

The co-inner-outer factorization is closely related to the inner-outer factorization. Given the inner-outer factorization (5.8) of $G(z)$, the co-inner-outer factorization of $\boldsymbol{H}(z) \equiv \boldsymbol{G}^{\mathrm{T}}(z)$ is given by equation (5.11). In words, the coinner and co-outer factors of $\boldsymbol{H}(z)$ are the transposed inner and outer factors of the transposed system $\boldsymbol{H}^{\mathrm{T}}(z)$.

$$
\boldsymbol{H}(z)=\boldsymbol{H}_{c o}(z) \boldsymbol{H}_{c i}(z)=\boldsymbol{G}_{o}^{\mathrm{T}}(z) \boldsymbol{G}_{i}^{\mathrm{T}}(z)
$$

Once again, if the co-inner factor $\boldsymbol{H}_{c i}(z)$ is not square, an orthogonal co-inner factor $\boldsymbol{H}_{c i}^{\perp}(z)$ exists, such that $\left[\boldsymbol{H}_{c i}^{*}(z) \boldsymbol{H}_{c i}^{\perp *}(z)\right]$ is a unitary system.

Note that, for SISO systems, the inner-outer and co-inner-outer factorization coincide. Moreover, the orthogonal (co-)inner factors do not exist for SISO systems.

\section{Causal Wiener filter continued}

Using these factorizations, the Causal Wiener filter $\boldsymbol{W}_{c}(z)$ can be expressed by equation (5.12) [Elliott (2000); Fraanje (2004); Nijsse (2006)]. Here, $\boldsymbol{P}_{\boldsymbol{d}}(z)$ and $\boldsymbol{T}_{\boldsymbol{d}}(z)$ are the combination of the primary path and tertiary path with the spectral factor $\boldsymbol{\Phi}_{\boldsymbol{d}}(z)$, see equation (5.13). The spectral factor $\boldsymbol{\Phi}_{\boldsymbol{d}}(z)$ is included to provide the proper frequency weighing in the mean squared error. Moreover, $\{\bullet\}_{+}$denotes the causality operator.

In appendix $C$, a proof of the optimality of the Causal Wiener filter is outlined, which is based on [Fraanje (2004), Appendix A].

$$
\begin{aligned}
& \boldsymbol{W}_{c}(z)=-\boldsymbol{S}_{o}^{\ddagger}(z)\left\{S_{i}^{*}(z) \boldsymbol{P}_{\boldsymbol{d}}(z) \boldsymbol{T}_{\boldsymbol{d}, c i}^{*}(z)\right\}_{+} \boldsymbol{T}_{\boldsymbol{d}, c o}^{\dagger}(z) \\
& \boldsymbol{P}_{\boldsymbol{d}}(z)=\boldsymbol{P}(z) \boldsymbol{\Phi}_{\boldsymbol{d}}(z), \quad \boldsymbol{T}_{\boldsymbol{d}}(z)=\boldsymbol{T}(z) \boldsymbol{\Phi}_{\boldsymbol{d}}(z)
\end{aligned}
$$

It can be seen that the Causal Wiener filter uses the outer factor $S_{o}(z)$ and the co-outer factor $T_{d, c o}(z)$ to causally invert as much of the dynamics of the secondary and tertiary paths as possible. The remaining dynamics of these paths can only be compensated by the anti-causal adjoint systems of the (co-) inner factors. To obtain a causal controller, the best causal approximation of these terms combined with the weighted primary path $\boldsymbol{P}_{\boldsymbol{d}}(z)$ is taken. 
The resulting minimum MSE value is given by equation (5.14), where $\{\bullet\}_{-}$ denotes the anti-causality operator.

$$
\begin{aligned}
& J_{\mathrm{MSE}}^{\mathrm{min}}=J_{\mathrm{MSE}}\left(\boldsymbol{W}_{c}\right)=\left\|\left\{\boldsymbol{S}_{i}^{*}(z) \boldsymbol{P}_{\boldsymbol{d}}(z) \boldsymbol{T}_{\boldsymbol{d}, c i}^{*}(z)\right\}_{-}\right\|_{2}^{+} \\
&\left\|\boldsymbol{S}_{i}^{*}(z) \boldsymbol{P}_{\boldsymbol{d}}(z) \boldsymbol{T}_{\boldsymbol{d}, c i}^{\perp *}(z)\right\|_{2}^{2}+\left\|S_{i}^{\perp *}(z) \boldsymbol{P}_{\boldsymbol{d}}(z)\right\|_{2}^{2}
\end{aligned}
$$

The three terms that constitute the minimum MSE value (5.14) can be given the following interpretation: the first term is due to non-minimum phase zeros and delays in $S(z)$ and $T(z)$ which are not cancelled by unstable poles and delays in the primary path $P(z)$.

The second term only exists when $T_{d, c i}^{\perp}(z)$ exists. For vibration isolation problems, this occurs when there are more disturbance sources than reference sensors. In this case, it is not possible to independently determine the contribution of all disturbance sources to the reference signals. Unless $\boldsymbol{P}(z)$ and $T_{c i}^{\perp}(z)$ are orthogonal, perfect cancellation can not be achieved. This orthogonality occurs only when, loosely speaking, the disturbance sources that are not measurable at the reference sensors do not cause any vibration at the error sensors. This is highly unlikely to be true in any practical situation.

Similarly, the last term only exists when $S_{i}^{\perp}(z)$ exists. For vibration isolation problems, this occurs when there are more error signals than actuator signals. It is clear that in that case perfect cancellation is not achievable, even if there are no delays and unstable zeros in $\boldsymbol{S}(z)$, unless $\boldsymbol{S}_{i}^{\perp}(z)$ and $\boldsymbol{P}_{\boldsymbol{d}}(z)$ are orthogonal. This required orthogonality of $\boldsymbol{S}_{i}^{\perp}(z)$ and $\boldsymbol{P}_{\boldsymbol{d}}(z)$ occurs when the disturbance sources only cause vibrations that can be controlled by the actuators. Again, this situation is unlikely to occur in practice.

\section{Illustrative example}

Let the primary path $P(z)$, the secondary path $S(z)$ and the tertiary path $T(z)$ be given by equations (5.15). Here, the values of $b_{1}$ and $c_{1}$ are such that $P(z)$ is stable. It is assumed that the disturbance signal $d(k)$ is white noise with unit variance, i.e. $\Phi_{d}(z)=1$.

$$
P(z)=\frac{a\left(z^{2}+b_{2} z+c_{2}\right)}{z\left(z^{2}+b_{1} z+c_{1}\right)}, \quad S(z)=\frac{1}{z}, \quad T(z)=\frac{1}{z}
$$

Note that $S(z)$ and $T(z)$ are pure delays and cannot be causally inverted. The inner-outer factorizations of these transfer paths are straightforward: the (co-) inner factors are given by the respective transfer paths and the outer factors are equal to 1 . The orthogonal (co-)inner factors do not exist.

Also, note that $P(z)$ is strictly proper with one delay, whereas the total delay in $S(z)$ and $T(z)$ is two samples. As a result, perfect cancellation can not be achieved. 
Through partial fraction expansion, it is easy to show that the Causal Wiener filter $W_{c}(z)$ is given by the last line in equation (5.16). The Causal Wiener filter is clearly stable and causal. Moreover, the minimum value of the performance measure is given by equation (5.17).

$$
\begin{gathered}
\begin{aligned}
W_{c}(z) & =-\{z P(z) z\}_{+}=-\left\{\frac{a z\left(z^{2}+b_{2} z+c_{2}\right)}{z^{2}+b_{1} z+c_{1}}\right\}_{+} \\
& =-\left\{a z+\frac{a\left(b_{2}-b_{1}\right) z^{2}+a\left(c_{2}-c_{1}\right) z}{z^{2}+b_{1} z+c_{1}}\right\}_{+} \\
& =-\frac{a\left(b_{2}-b_{1}\right) z^{2}+a\left(c_{2}-c_{1}\right) z}{z^{2}+b_{1} z+c_{1}} \\
J_{\mathrm{MSE}}\left(W_{c}\right) & =\left\|\left\{S_{i}^{*}(z) P(z) T_{c i}^{*}(z)\right\}_{-}\right\|_{2}^{2}=\|a z\|_{2}^{2}=a^{2}
\end{aligned}
\end{gathered}
$$

\subsubsection{Effects of measurement noise}

In this section, the effects of additive measurement noise (at both the reference sensors and error sensors) on the Causal Wiener filter are studied. For this purpose, the feedforward system model is augmented with noise signals, as shown in figure 5.5. Here, $\boldsymbol{n}_{r}(k), \boldsymbol{n}_{e}(k)$ are white noise signals with unit variance and $\boldsymbol{\Phi}_{r}(z), \boldsymbol{\Phi}_{e}(z)$ are their respective spectral factors. It is assumed that all the noise components in $\boldsymbol{n}_{r}(k)$ are uncorrelated with the components in $\boldsymbol{n}_{e}(k)$. Note that, if necessary, any correlated parts of the noise signals can be incorporated into $d(k)$.

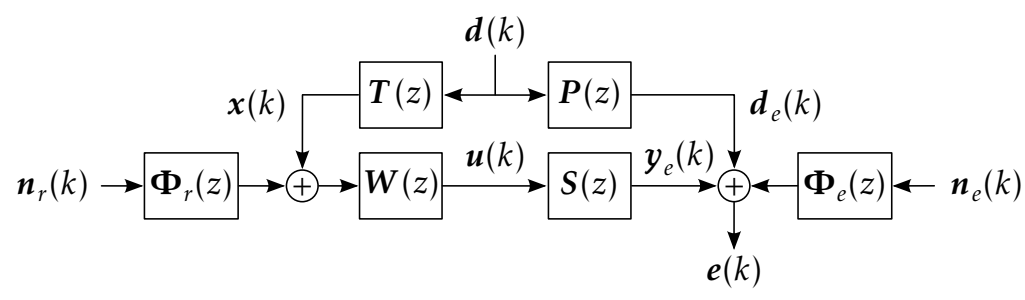

Figure 5.5: System model including measurement noise contributions on the reference and error sensors 
The optimal control problem with measurement noise can be recast to the original, noiseless problem (5.5), by using the change of variables as defined by equation (5.18).

$$
\tilde{\boldsymbol{d}}(k)=\left[\begin{array}{c}
\boldsymbol{d}(k) \\
\boldsymbol{n}_{r}(k) \\
\boldsymbol{n}_{e}(k)
\end{array}\right]
$$

With this change in variables, the augmented primary and tertiary paths are given by equations (5.19) and (5.20). Note that the original primary and tertiary path are combined with the spectral factor of the disturbance signal, see also equation (5.13).

$$
\begin{aligned}
& \tilde{\boldsymbol{P}}(z)=\left[\begin{array}{lll}
\boldsymbol{P}_{\boldsymbol{d}}(z) & \mathbf{0} & \boldsymbol{\Phi}_{e}(z)
\end{array}\right] \\
& \tilde{\boldsymbol{T}}(z)=\left[\begin{array}{lll}
\boldsymbol{T}_{\boldsymbol{d}}(z) & \boldsymbol{\Phi}_{r}(z) & 0
\end{array}\right]
\end{aligned}
$$

The co-inner-outer factorization of $\tilde{\boldsymbol{T}}(z)$ is now given by equation (5.21), where the partitioning of the co-inner factor is compatible with the partitioning in equation (5.20). Moreover, the spectrum of the augmented tertiary path is given by equation (5.22).

$$
\begin{aligned}
& \tilde{\boldsymbol{T}}(z)=\tilde{\boldsymbol{T}}_{c o}(z) \tilde{\boldsymbol{T}}_{c i}(z)=\tilde{\boldsymbol{T}}_{c o}(z)\left[\begin{array}{lll}
\tilde{\boldsymbol{T}}_{1 c i}(z) & \tilde{\boldsymbol{T}}_{2 c i}(z) & \mathbf{0}
\end{array}\right] \\
& \tilde{\boldsymbol{T}}(z) \tilde{\boldsymbol{T}}^{*}(z)=\tilde{\boldsymbol{T}}_{c o}(z) \tilde{\boldsymbol{T}}_{c o}^{*}(z)=\boldsymbol{T}_{\boldsymbol{d}}(z) \boldsymbol{T}_{\boldsymbol{d}}^{*}(z)+\boldsymbol{\Phi}_{r}(z) \boldsymbol{\Phi}_{r}^{*}(z)
\end{aligned}
$$

This offers the following interpretation: loosely speaking, the "magnitude" of the co-outer factor is increased (compared to the noiseless case), especially at frequencies where the reference noise spectrum is significant. Because the original tertiary path can be factored as $\boldsymbol{T}(z)=\tilde{\boldsymbol{T}}_{c o}(z) \tilde{\boldsymbol{T}}_{1 c i}(z)$, the "magnitude" of $\tilde{T}_{1 c i}(z)$ (and its adjoint) is reduced. ${ }^{3}$

The Causal Wiener filter is now given by equation (5.23). Because the "magnitudes" of $\tilde{\boldsymbol{T}}_{c o}^{\dagger}(z)$ and $\tilde{\boldsymbol{T}}_{1 c i}^{*}(z)$ are reduced, the "magnitude" of the Causal Wiener filter is reduced as well. This is intuitively correct, as at frequencies where the reference signal contains little or no information about the disturbance sources, the controller gain should be reduced.

$$
\tilde{\boldsymbol{W}}_{c}(z)=-\boldsymbol{S}_{o}^{\ddagger}(z)\left\{\boldsymbol{S}_{i}^{*}(z) \boldsymbol{P}_{\boldsymbol{d}}(z) \tilde{\boldsymbol{T}}_{1 c i}^{*}(z)\right\}_{+} \tilde{\boldsymbol{T}}_{c o}^{\dagger}(z)
$$

3 Note that $\tilde{T}_{1 c i}(z)$ by itself is no longer an inner factor. 
Due to the presence of the noise signals, the orthogonal co-inner factor $\tilde{T}_{c i}^{\perp}(z)$ necessarily exists ${ }^{4}$ and can be expressed by equation (5.24), where $\tilde{T}_{1 c i}^{\perp}(z)$ and $\tilde{\boldsymbol{T}}_{2 c i}^{\perp}(z)$ are such that $\left[\tilde{T}_{c i}^{*}(z) \tilde{\boldsymbol{T}}_{c i}^{\perp *}(z)\right]$ is unitary.

$$
\tilde{\boldsymbol{T}}_{c i}^{\perp}(z)=\left[\begin{array}{ccc}
\tilde{\boldsymbol{T}}_{1 c i}^{\perp}(z) & \tilde{\boldsymbol{T}}_{2 c i}^{\perp}(z) & \mathbf{0} \\
\mathbf{0} & \mathbf{0} & \boldsymbol{I}_{N_{e}}
\end{array}\right]
$$

Using this partitioning for $\tilde{\boldsymbol{T}}_{c i}^{\perp}(z)$, the MSE cost criterion is given by equation (5.25), which is in a similar form as the noiseless solution (5.14). The first two terms are changed due to the measurement noise on the reference sensors. Another notable difference is the presence of the noise spectrum on the error signals. This is as expected, because a feedforward controller can obviously never compensate for disturbances that are uncorrelated with the reference signal.

$$
\begin{gathered}
J_{\mathrm{MSE}}\left(\tilde{\boldsymbol{W}}_{c}\right)=\left\|\left\{S_{i}^{*}(z) \boldsymbol{P}_{\boldsymbol{d}}(z) \tilde{\boldsymbol{T}}_{1 c i}^{*}(z)\right\}_{-}\right\|_{2}^{2}+\left\|\boldsymbol{S}_{i}^{*}(z) \boldsymbol{P}_{\boldsymbol{d}}(z) \tilde{\boldsymbol{T}}_{1 c i}^{\perp *}(z)\right\|_{2}^{2} \\
+\left\|\boldsymbol{S}_{i}^{\perp *}(z) \boldsymbol{P}_{\boldsymbol{d}}(z)\right\|_{2}^{2}+\left\|\boldsymbol{\Phi}_{e}(z)\right\|_{2}^{2}
\end{gathered}
$$

\section{Illustrative example (continued)}

Consider the example of equation (5.15) once more, but with measurement noise included. The noise signals on the reference measurement and error measurement are white noise signals with variance $\sigma_{n_{r}}^{2}$ and $\sigma_{n_{e}}^{2}$ respectively, see equation (5.26).

$$
\Phi_{r}(z)=\sigma_{n_{r}} \quad \Phi_{e}(z)=\sigma_{n_{e}}
$$

The augmented tertiary path is expressed by equation (5.27). The co-outer factor is then found from equation (5.28).

$$
\begin{aligned}
& \tilde{T}(z)=\left[\begin{array}{lll}
\frac{1}{z} & \sigma_{n_{r}} & 0
\end{array}\right] \\
& \tilde{T}_{c o}(z) \tilde{T}_{c o}^{*}(z)=\tilde{T}(z) \tilde{T}^{*}(z)=1+\sigma_{n_{r}}^{2} \Rightarrow \tilde{T}_{c o}(z)=\sqrt{1+\sigma_{n_{r}}^{2}}
\end{aligned}
$$

4 There are more disturbance sources than reference sensors now 
As a result, the co-inner-outer factorization of $\tilde{T}(z)$ is given by equation (5.29) and the orthogonal co-inner factor can be shown to be given by equation (5.30).

$$
\begin{aligned}
& \tilde{T}(z)=\tilde{T}_{c o}(z) \tilde{T}_{c i}(z)=\sqrt{1+\sigma_{n_{r}}^{2}}\left[\frac{1}{z \sqrt{1+\sigma_{n_{r}}^{2}}} \quad \frac{\sigma_{n_{r}}}{\sqrt{1+\sigma_{n_{r}}^{2}}} \quad 0\right] \\
& \tilde{T}_{c i}^{\perp}(z)=\left[\begin{array}{ccc}
-\frac{\sigma_{n_{r}}}{z \sqrt{1+\sigma_{n_{r}}^{2}}} & \frac{1}{\sqrt{1+\sigma_{n_{r}}^{2}}} & 0 \\
0 & 0 & 1
\end{array}\right]
\end{aligned}
$$

The Causal Wiener filter of equation (5.23) can now be evaluated, see equation (5.31). Clearly, the controller gain is reduced over all frequencies in comparison to the noiseless Causal Wiener filter (equation (5.16)).

$$
\tilde{W}_{c}(z)=-\left\{z P(z) \frac{z}{\sqrt{1+\sigma_{n_{r}}^{2}}}\right\}_{+} \frac{1}{\sqrt{1+\sigma_{n_{r}}^{2}}}=\frac{W(z)}{1+\sigma_{n_{r}}^{2}}
$$

The minimum value of the MSE cost criterion (5.25) is expressed by equation (5.32). Because in general the "open loop" MSE is larger than the feedforward controlled MSE (i.e. $\|P(z)\|_{2}^{2}>a^{2}$ ), this minimum value is larger than the minimum value for the noiseless case $a^{2}$. Also note that for $\sigma_{n_{r}}^{2} \rightarrow 0$ and $\sigma_{n_{e}}^{2} \rightarrow 0$, the noiseless solution (5.17) is obtained.

$$
\begin{aligned}
J_{\mathrm{MSE}}\left(\tilde{W}_{c}\right) & =\left\|\left\{z P(z) \frac{z}{\sqrt{1+\sigma_{n_{r}}^{2}}}\right\}_{-}\right\|_{2}^{2}+\left\|z P(z) \cdot-\frac{\sigma_{n_{r}} z}{\sqrt{1+\sigma_{n_{r}}^{2}}}\right\|_{2}^{2}+\left\|H_{e}\right\|_{2}^{2} \\
& =\frac{a^{2}}{1+\sigma_{n_{r}}^{2}}+\frac{\sigma_{n_{r}}^{2}}{1+\sigma_{n_{r}}^{2}}\|P(z)\|_{2}^{2}+\sigma_{n_{e}}^{2}
\end{aligned}
$$

\subsubsection{Control effort weighing}

An optimization problem that is closely related to the noise corrupted problem is formulated by incorporating the frequency-weighted control signal in the error signal, see equation (5.33). Here, $\boldsymbol{u}_{w}(k)$ denotes the weighted control signal, i.e. after filtering by the weighing filter $S_{\text {reg }}(z)$, see equation (5.34).

$$
\begin{aligned}
& J_{\mathrm{MSE}}(\boldsymbol{W})=\|\boldsymbol{e}(k)\|_{\mathrm{RMS}}^{2}+\left\|\boldsymbol{u}_{w}(k)\right\|_{\mathrm{RMS}}^{2} \\
& \boldsymbol{U}_{w}(z)=\boldsymbol{S}_{\mathrm{reg}}(z) \boldsymbol{U}(z)
\end{aligned}
$$

This approach is useful in cases where the control effort must be restricted in certain frequency ranges. In chapter 8 , the Causal Wiener filter with regularization of the control effort is computed as a realistic prediction for the vibration isolation performance. 
To recast this problem to the standard form of section 5.2.2, an augmented error signal is introduced, see equation (5.35).

$$
\overline{\boldsymbol{e}}(k)=\left[\begin{array}{c}
\boldsymbol{e}(k) \\
\boldsymbol{u}_{w}(k)
\end{array}\right]
$$

The resulting augmented (or regularized) primary path and secondary path are expressed in equation (5.36). The inner factor $\bar{S}_{i}(z)$ of the regularized secondary path $\bar{S}(z)$ and the corresponding orthogonal inner factor $\bar{S}_{i}^{\perp}(z)$ are factorized as shown in equation (5.37).

$$
\begin{array}{ll}
\overline{\boldsymbol{P}}(z)=\left[\begin{array}{c}
\boldsymbol{P}_{\boldsymbol{d}}(z) \\
\mathbf{0}
\end{array}\right], & \overline{\boldsymbol{S}}(z)=\left[\begin{array}{c}
\boldsymbol{S}(z) \\
S_{\mathrm{reg}}(z)
\end{array}\right] \\
\overline{\boldsymbol{S}}_{i}(z)=\left[\begin{array}{l}
\overline{\boldsymbol{S}}_{1 i}(z) \\
\overline{\boldsymbol{S}}_{2 i}(z)
\end{array}\right], & \overline{\boldsymbol{S}}_{i}^{\perp}(z)=\left[\begin{array}{l}
\overline{\boldsymbol{S}}_{1 i}^{\perp}(z) \\
\overline{\boldsymbol{S}}_{2 i}^{\perp}(z)
\end{array}\right]
\end{array}
$$

Using these definitions, the Causal Wiener filter is given by equation (5.38) and the minimal value of the mean squared error is given by equation (5.39).

$$
\begin{gathered}
\overline{\boldsymbol{W}}_{c}(z)=-\overline{\boldsymbol{S}}_{o}^{\ddagger}(z)\left\{\overline{\boldsymbol{S}}_{1 i}^{*}(z) \boldsymbol{P}_{\boldsymbol{d}}(z) \boldsymbol{T}_{\boldsymbol{d}, c i}^{*}(z)\right\}_{+} \boldsymbol{T}_{\boldsymbol{d}, c o}^{+}(z) \\
J_{\mathrm{MSE}}\left(\overline{\boldsymbol{W}}_{c}\right)=\left\|\left\{\overline{\boldsymbol{S}}_{1 i}^{*}(z) \boldsymbol{P}_{\boldsymbol{d}}(z) \boldsymbol{T}_{\boldsymbol{d}, c i}^{*}(z)\right\}_{-}\right\|_{2}^{2}+\left\|\overline{\boldsymbol{S}}_{1 i}^{*}(z) \boldsymbol{P}_{\boldsymbol{d}}(z) \boldsymbol{T}_{\boldsymbol{d}, c i}^{\perp *}(z)\right\|_{2}^{2} \\
+\left\|\overline{\boldsymbol{S}}_{1 i}^{\perp *}(z) \boldsymbol{P}_{\boldsymbol{d}}(z)\right\|_{2}^{2}+\left\|\boldsymbol{\Phi}_{e}(z)\right\|_{2}^{2}
\end{gathered}
$$

\subsubsection{Conclusions and final remarks}

In the preceding sections, the Causal Wiener filter has been presented, which is the causal, stable feedforward controller that minimizes the mean squared error. It was shown that the minimal mean squared error is made up of terms related to the control system structure (i.e. these terms depend on the number of actuators, error sensors, reference sensors and disturbance sources) and one term that is related to the non-minimum phase zeros and delays in the system.

It has been shown that measurement noise on the error signals that is uncorrelated with the reference signals can not be compensated for. Actually, this holds for any disturbance source that is uncorrelated with the reference signals. Moreover, the effect of measurement noise on the reference signals is shown to reduce the feedforward gain (and therefore performance), especially at frequencies where the noise dominates. 
As a consequence, the noise levels for the error sensors and the reference sensors have a significant effect on the overall system performance. In chapter 6 , various sensor types are evaluated for their applicability in vibration isolation problems.

The MSE cost criterion can be extended to include frequency-weighted errors and control effort weighing. It has been demonstrated that these extended minimization problems can be recast to the original form. As a result, these problems are also solvable with the theory that has been discussed in this section.

Lastly, it is obvious from e.g. equation (5.12) that the primary path $\boldsymbol{P}(z)$, the secondary path $S(z)$ and the tertiary path $T(z)$ (as well as the spectral factors of the disturbance signal $\boldsymbol{d}$ and the noise signals $\boldsymbol{n}_{e}$ and $\boldsymbol{n}_{r}$ ) have to be known to compute the Causal Wiener filter. In practice, this will require some type of system identification to be performed. However, in many cases, the disturbance sources may not be under the user's control, which means that identification of $\boldsymbol{P}(z)$ and $\boldsymbol{T}(z)$ is not possible.

To circumvent the difficulties concerning the identification of $\boldsymbol{P}(z)$ and $\boldsymbol{T}(z)$, an adaptive scheme is used to find an approximation of the optimal feedforward controller. Moreover, the adaptive nature of the controller offers (to some extent) tracking capabilities for time-varying disturbances and system behaviour. In the following section, the chosen adaptive feedforward control method is presented.

\subsection{Adaptive feedforward control}

Adaptive filtering has been a research topic for many decades and a large body of literature is available on the subject. Due to the advent of fast and (relatively) cheap digital computers, the number of industrial applications of adaptive filtering methods has also increased rapidly. Areas of application include, but are not limited to, speech and signal processing, motion control (i.e. flight control or robotics), process control and active noise control.

A complete review of adaptive filter theory is beyond the scope of this thesis. Instead, the discussion will be limited to discrete-time adaptive filter theory. A general treatment of discrete-time adaptive filters is available in many textbooks e.g. [Widrow and Stearns (1985); Landau et al. (1998); Haykin (2002); Sayed (2003)].

More specifically, we limit ourselves to the theory of adaptive filters that are widely used in (broadband) Active Noise Control (ANC), as this field of application is closely related to vibration isolation. An introduction to the subject of active noise (and vibration) control can be found in e.g. [Fuller et al. 
(1997); Hansen and Snyder (1997)]. The theory and application of adaptive filters in active noise control is treated extensively in these textbooks: [Kuo and Morgan (1996); Elliott (2001)], and in the tutorial paper by [Kuo and Morgan (1999)].

\subsubsection{Filtered-X LMS algorithm}

A widely used adaptive algorithms in ANC applications is the filtered-reference (or filtered-x) least-mean-squares (FxLMS) algorithm, which forms the basis for the adaptive feedforward control used in this thesis. Most of the details in this section can be found in the excellent textbook by [Sayed (2003)]. In this section, the discussion is restricted to scalar signals and SISO systems. The FxLMS algorithm is then motivated from the adaptive feedforward control problem shown in figure 5.6. It is very similar to the problem as depicted in figure 5.4, with the exception of the time-variant nature of the feedforward controller $W(k)$, as indicated by the arrow.

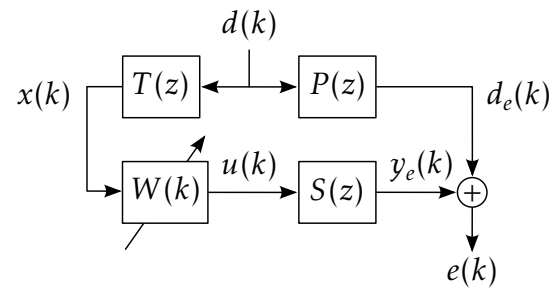

Figure 5.6: Adaptive feedforward control problem

In section 5.2.1, the mean squared error is used as the cost criterion in the derivation of the Causal Wiener controller. To motivate the FxLMS algorithm, this cost criterion is approximated by the instantaneous squared error (ISE), see equation (5.40).

$$
J_{\text {ISE }}(k)=e(k)^{\mathrm{T}} e(k)
$$

Moreover, it is assumed that the feedforward control signal can be computed by the linear regression expression (5.41). Here, $w(k)$ is a time-varying column vector of controller weights (with $L$ the user-definable number of components) and $\psi(k)$ is a regression vector. Note that, due to the linear parametrization, the quadratic cost criterion (5.40) has a unique global minimum.

$$
u(k)=w^{\mathrm{T}}(k) \psi(k)=\sum_{l=1}^{L} w_{l}(k) \psi_{l}(k)
$$


The regression vector $\psi(k)$ is obtained from the reference signal $x(k)$ by filtering $x(k)$ through an $L \times 1$ LTI filter $\boldsymbol{F}(z)$, see equation (5.42).

$$
\psi(k)=\boldsymbol{F}[x(k)]
$$

Here, $\boldsymbol{F}[\cdot]$ indicates the filtering operation in the time-domain (convolution). Note that, for the moment, no other assumptions than linearity and timeinvariance are made regarding $\boldsymbol{F}(z)$. Some possible choices for the structure of the reference filter $\boldsymbol{F}(z)$ are discussed in section 5.3.5.

The controller weight vector $w(k)$ is updated according to a gradient-search algorithm, see equation (5.43). Here, $\mu(k)$ is a user-defined, possibly timevarying parameter that controls the update step size.

$$
w(k+1)=w(k)-\frac{\mu(k)}{2}\left(\frac{\partial J_{\mathrm{ISE}}(k)}{\partial w}\right)^{\mathrm{T}}=w(k)-\mu(k)\left(\frac{\partial e(k)}{\partial w}\right)^{\mathrm{T}} e(k)
$$

The difficulty lies in obtaining the gradient of the error signal with respect to the controller weights. The error signal is expressed by equation (5.44). Note that the last term denotes the filtering of the output of the time-varying controller by the time-invariant secondary path $S(z)$. It is generally not possible to find a closed form expression for the gradient of this term with respect to the controller weights $w$.

$$
e(k)=d_{e}(k)+y_{e}(k)=d_{e}(k)+S[u(k)]=d_{e}(k)+S\left[w^{\mathrm{T}}(k) \psi(k)\right]
$$

Therefore, it is common to use a slow adaptation assumption, i.e. it is assumed that the controller weights vary slowly with respect to the dynamics of the secondary path $S(z)$. In this case, the error signal may be approximated by equation (5.45), where the order of the secondary path $S(z)$ and the feedforward controller are reversed. Here, $S \otimes[\psi(k)]$ denotes the filtering of all signal components in $\psi(k)$ by the filter $S(z)$.

$$
e(k) \approx d_{e}(k)+w^{\mathrm{T}}(k)(S \otimes[\psi(k)])
$$

Using this approximation and the equivalent form (5.46) for $S \otimes[\psi(k)]^{5}$, the update rule for the controller weights reduces to equation (5.47).

$$
\begin{aligned}
& \psi_{S}(k)=S \otimes[\psi(k)]=F[S[x(k)]] \\
& w(k+1)=w(k)-\mu(k) \psi_{S}(k) e(k)
\end{aligned}
$$

5 The last equality follows directly from the linearity and time-invariance of $\boldsymbol{F}(z)$ and $S(z)$ 


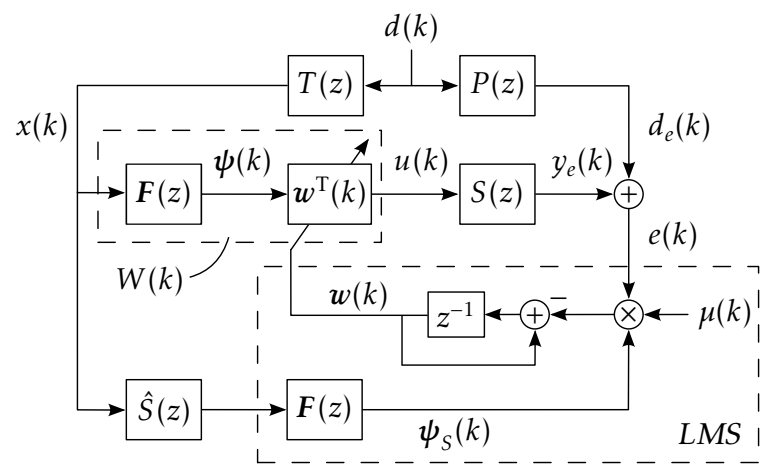

Figure 5.7: Block diagram showing the FXLMS algorithm (with time-variant step size $\mu(k))$, showing the internal structure of the adaptive controller $W(k)$ and the underlying LMS update law with time-varying step size $\mu(k)$ (denoted by LMS).

With a constant step size parameter $\mu(k)=\mu$, equation (5.47) is the standard filtered-reference LMS (FxLMS) algorithm. The name follows from the fact that the reference signal $x(k)$ is filtered by $S(z)$ before it is used in the LMS algorithm for the computation of the regression vector $\psi_{S}(k)$.

A block diagram showing the structure of the FxLMS adaptation algorithm is shown in figure 5.7. Note that in practice, the secondary path $S(z)$ is unknown and an approximate model $\hat{S}(z)$ should be used instead, as indicated in figure 5.7. However, for the moment, perfect plant knowledge is assumed.

\section{Stability}

The stability of the LMS algorithm (and any derived algorithm like FxLMS) depends on the user-defined parameter $\mu(k)$ and the spectral properties of the (filtered) regression vector, see e.g. [Elliott (2000); Haykin (2002)].

According to e.g. [Rupp and Sayed (1996) ${ }^{6}$, the FxLMS algorithm is stable in the sense that it converges in the mean to the global minimum and it converges in the mean-square ${ }^{7}$, when the time-varying step size $\mu(k)$ is computed according to equation (5.48). Here, $\epsilon$ is a (usually small) number that regularizes the quotient and $\bar{\mu}$ is a user-defined constant normalized step size.

$$
\mu(k)=\frac{\bar{\mu}}{\epsilon+\left\|\psi_{S}(k)\right\|_{2}^{2}}, \quad 0<\bar{\mu}<2, \quad \epsilon>0
$$

6 See also [Sayed (2003), Ch. 9 and App. 9.B] for an extensive treatment

7 This is equivalent to the notion of bounded-input, bounded-output (BIBO) stability in linear systems theory. 
The LMS algorithm with this choice for $\mu(k)$ is called $(\epsilon-)$ normalized LMS or $(\epsilon-)$ NLMS. The FxLMS algorithm with time-varying step size (5.48) will be referred to as $\epsilon$-FxNLMS in this thesis.

An important advantage over the standard FxLMS algorithm is that the choice of $\bar{\mu}$ (and hence stability) is independent of the properties of the regression vector $\psi_{S}(k)$. This comes at the expense of an increased computational complexity, as equation (5.48) must be evaluated at each sample interval.

The steady state mean squared error for the $\epsilon-$ FxNLMS algorithm can be approximated by equation (5.49), see [Sayed (2003), Ch. 6]. Here, $\sigma_{n}^{2}$ denotes the variance of the part of the disturbance signal $d_{e}(k)$ that cannot be compensated for by the feedforward controller. Note that $\sigma_{n}^{2}$ can be interpreted as the mean squared error of the Causal Wiener controller (see section 5.2.2).

It is then clear that the $\epsilon-\mathrm{FxNLMS}$ algorithm has an excess mean squared error compared to the Causal Wiener controller performance, which is typical for any adaptive algorithm. Moreover, the choice for $\bar{\mu}$ also determines the excess mean squared error, aside from the stability of the adaptation as discussed above.

$$
J_{\mathrm{MSE}}=\lim _{k \rightarrow \infty} \mathrm{E}\left\{J_{\mathrm{ISE}}(k)\right\} \approx \sigma_{n}^{2}\left(1+\frac{\bar{\mu}}{2-\bar{\mu}}\right)
$$

\section{Convergence rate}

For the FxLMS algorithm (fixed stepsize $\mu(k)=\mu$ ) and under assumption of slow adaptation, it can be shown that the weight update (5.47) is governed (in the mean) by first order modes with eigenvalues $1-\mu \lambda_{i}$. Here, $\lambda_{i}$ are the eigenvalues of the autocorrelation matrix $R_{\psi_{S} \psi_{S}}$ of the filtered regression vector $\psi_{S}(k)$, see equation (5.50). Therefore, the choice for the step size $\mu$ also influences the convergence rate of the adaptation, with larger step sizes generally leading to faster convergence. ${ }^{8}$

$$
\boldsymbol{R}_{\psi_{S} \psi_{S}}=\mathrm{E}\left\{\psi_{S}(k) \psi_{S}(k)^{\mathrm{T}}\right\}
$$

In e.g. [Elliott (2000)], it is argued that the eigenvalue spread of $\boldsymbol{R}_{\psi_{S} \psi_{S}}$ determines the ratio of the maximum and minimum time constants $\tau_{\max }$ and $\tau_{\min }$ for the FxLMS algorithm, see equation (5.51).

$$
\frac{\tau_{\max }}{\tau_{\min }} \approx \frac{\lambda_{\max }}{\lambda_{\min }}
$$

8 See [Sayed (2003), Sect. 4.3] for a critical review on the effect of the step size on the overall convergence rate. 


$$
\frac{\lambda_{\max }}{\lambda_{\min }} \leq \frac{\max _{-\pi \leq \omega \leq \pi} \operatorname{tr}\left(\boldsymbol{F}\left(\mathrm{e}^{j \omega}\right) S\left(\mathrm{e}^{j \omega}\right) P_{x x}(\omega) S\left(\mathrm{e}^{j \omega}\right)^{\mathrm{H}} \boldsymbol{F}\left(\mathrm{e}^{j \omega}\right)^{\mathrm{H}}\right)}{\min _{-\pi \leq \omega \leq \pi} \operatorname{tr}\left(\boldsymbol{F}\left(\mathrm{e}^{j \omega}\right) S\left(\mathrm{e}^{j \omega}\right) P_{x x}(\omega) S\left(\mathrm{e}^{j \omega}\right)^{\mathrm{H}} \boldsymbol{F}\left(\mathrm{e}^{j \omega}\right)^{\mathrm{H}}\right)}
$$

An upper bound for the eigenvalue spread is given by equation $(5.52)^{9}$ and this bound becomes tight for large filters, i.e. for large values of the number of controller weights $L$.

Expression (5.52) offers the following physical interpretation: relatively slow convergence of some modes can be expected when either of the following conditions occur:

i. The power spectral density $P_{x x}(\omega)$ of the reference signal has a large dynamic range, i.e. the reference signal is not white, but coloured.

ii. The magnitude frequency response of the secondary path $S(z)$ has a large dynamic range. Note that the dynamic range of $S(z)$ may be reduced by applying feedback control.

iii. The magnitude frequency response of any of the channels in the regression filter $\boldsymbol{F}(z)$ has a large dynamic range.

Note that slow convergence is not necessarily troublesome, e.g. when a slowly converging mode has only little influence on the overall performance.

According to [An et al. (1997)], the convergence rate of the NLMS algorithm can be much larger than that for standard LMS, because the regression vector is effectively normalized, thus reducing its dynamic range. However, the reported improvement is most notable for relatively small numbers of controller weights $L$, and is derived for a Gaussian regression vector. In practice, this assumption may be violated and the overall convergence rate may still be unacceptably slow, especially for large filters. In the following section, two modifications to the FxLMS algorithm are described that improve the convergence rate of the adaptation.

\subsubsection{Improving the convergence rate}

In this section, two modifications to the FxLMS algorithm are discussed that result in an improvement convergence rate.

9 This follows from the fact that eigenvalues of any matrix are bounded in magnitude by the smallest and largest singular value of that matrix, and the relationship between the frequency response and the singular values for linear systems. 


\section{Preconditioned FxLMS}

The first modification to the FxLMS algorithm, called preconditioned FxLMS, has been proposed by [Elliott and Cook (2000)]. The preconditioned FxLMS algorithm is illustrated in figure 5.8.

The key idea of the preconditioned FxLMS algorithm is that, by incorporating the inverse outer factor $\hat{S}_{o}^{-1}(z)$, the reference signal only has to be filtered by the inner factor $\hat{S}_{i}(z)$. Because the inner factor is all-pass, it no longer plays a role in the eigenvalue spread of equation (5.52). When the original dynamic range of $\hat{S}(z)$ is large, the overall convergence rate is greatly increased.

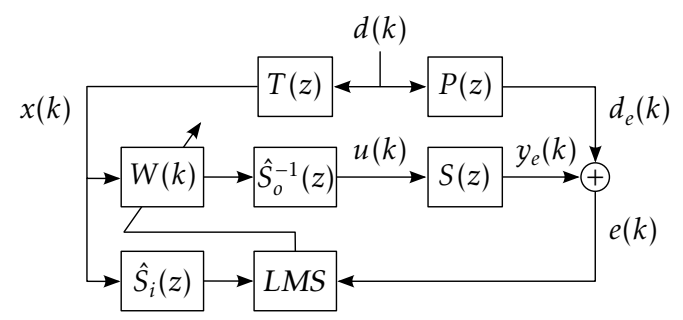

Figure 5.8: Preconditioned FxLMS algorithm [Elliott and Cook (2000)]

\section{Modified FxLMS}

Recall from the discussion on page 72 that the derivation of the FxLMS algorithm is based on the assumption of slow adaptation, i.e. the equivalence of the left-hand and right-hand sides of equation (5.53).

$$
S\left[w^{\mathrm{T}}(k) \psi(k)\right] \approx w^{\mathrm{T}}(k)(S \otimes[\psi(k)])\left(=w^{\mathrm{T}}(k) \psi_{S}(k)\right)
$$

Bjarnason (1992) proposed the modified FxLMS algorithm that circumvents the slow adaptation requirement by using an internal model, see also e.g. [Rupp and Sayed (1998)]. This algorithm is also sometimes referred to as swapping of plant and controller. The modified FxLMS algorithm is illustrated in figure 5.9.

The LMS adaptation now uses the modified error signal $\hat{e}(k)$, see also equation (5.54). Assuming perfect knowledge of the secondary path, it is easily recognized that the modified error $\hat{e}(k)$ has the same form as equation (5.45), which is the starting point of the derivation of the FxLMS algorithm.

$$
\begin{aligned}
\hat{e}(k) & =e(k)-\hat{S}\left[w^{\mathrm{T}}(k) \psi(k)\right]+w^{\mathrm{T}}(k) \psi_{S}(k) \\
& =d_{e}(k)+S\left[w^{\mathrm{T}}(k) \psi(k)\right]-\hat{S}\left[w^{\mathrm{T}}(k) \psi(k)\right]+w^{\mathrm{T}}(k) \psi_{S}(k)
\end{aligned}
$$




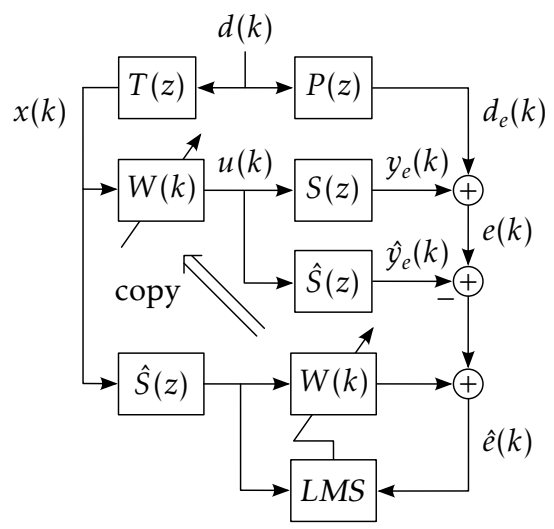

Figure 5.9: Modified FXLMS algorithm [Bjarnason (1992)]

The faster convergence of this algorithm follows from the fact that (with perfect plant knowledge) the modified error $\hat{e}(k)$ only depends on the current weight vector $w(k)$, whereas the original error $e(k)$ also depends on previous values of the weight vector. As a result, the update direction for the weight vector is closer to the optimal direction, which speeds up the convergence.

The computational complexity of the modified FxLMS algorithm is larger than the FxLMS algorithm, because of the additional evaluation of the secondary path model $\hat{S}(z)$ and the controller $W(k)$.

Note that the preconditioning of figure 5.8 can be combined with the modified FxLMS algorithm, leading to even faster convergence. Moreover, because the inner factor $\hat{S}_{i}(z)$ is usually of lesser order than $\hat{S}(z)$, the increase in computational complexity is less significant.

\subsubsection{Effects of errors in the secondary path model}

In practice, the secondary path model $\hat{S}(z)$ is not a perfect representation of the secondary path. In this case, the convergence in the mean of the FxLMS weight update is governed by the eigenvalues $\tilde{\lambda}_{i}$ of the cross-correlation matrix $C_{\psi_{\hat{S}} \psi_{S}}$, see equation (5.55), instead of the eigenvalues of the autocorrelation matrix $\boldsymbol{R}_{\psi_{S}} \psi_{S}$.

$$
C_{\psi_{\hat{S}} \psi_{S}}=\mathrm{E}\left\{\psi_{\hat{S}}(k) \psi_{S}(k)^{\mathrm{T}}\right\}=\mathrm{E}\left\{\hat{S}[\psi(k)](S[\psi(k)])^{\mathrm{T}}\right\}
$$

In general, these eigenvalues $\tilde{\lambda}_{i}$ are complex, instead of real and non-negative as is the case for the autocorrelation matrix $R_{\psi_{S}} \psi_{S}$. The stability requirement for the FxLMS algorithm is now given by equation (5.56).

$$
0<\mu<2 \operatorname{Re}\left(\tilde{\lambda}_{i}\right) /\left|\tilde{\lambda}_{i}\right|^{2}, \quad \forall \tilde{\lambda}_{i}
$$


Note that, for any stabilizing $\mu$ to exist, all eigenvalues $\tilde{\lambda}_{i}$ must have positive real parts, i.e. $C_{\psi_{\hat{S}} \psi_{S}}$ must be positive definite. A sufficient condition (assuming slow adaptation) is the strictly positive real (SPR) condition (5.57) [Wang and Ren (1999)].

$$
\hat{S}^{\mathrm{H}}\left(\mathrm{e}^{j \omega}\right) S\left(\mathrm{e}^{j \omega}\right)+S^{\mathrm{H}}\left(\mathrm{e}^{j \omega}\right) \hat{S}\left(\mathrm{e}^{j \omega}\right)>0, \quad \forall \omega,-\pi \leq \omega \leq \pi
$$

For SISO systems, equation (5.57) implies that a stabilizing step size $\mu>0$ exists only if $-90^{\circ}<\Delta \phi(\omega)<90^{\circ}$. Here, $\Delta \phi(\omega)$ is the phase error between $S\left(\mathrm{e}^{j \omega}\right)$ and $\hat{S}\left(\mathrm{e}^{j \omega}\right)$. Similar results have been obtained for the modified FxLMS algorithm [Lopes and Piedade (2004)]. However, for both algorithms, the largest stabilizing step size may be very small, resulting in greatly reduced convergence rates.

\section{Improving FxLMS robustness to modelling errors}

By applying a leakage factor $\beta>0$ in the FxLMS weight update, as indicated in equation (5.58), the stability requirement (5.56) is altered to equation (5.59). Clearly, by choosing $\beta$ large enough, a stabilizing step size $\mu$ can always be found that satisfies the augmented stability requirement. The update law (5.58) is widely known as leaky FxLMS.

$$
\begin{aligned}
& w(k+1)=(1-\mu \beta) w(k)-\mu \psi_{S}(k) e(k) \\
& 0<\mu<2 \operatorname{Re}\left(\tilde{\lambda}_{i}+\beta\right) /\left|\tilde{\lambda}_{i}+\beta\right|^{2}, \quad \forall \tilde{\lambda}_{i}
\end{aligned}
$$

Note that the leaky FxLMS algorithm is usually derived from the augmented cost criterion (5.60), in which the weighted 2-norm of the weight vector is added to the instantaneous squared error.

As a result, the weight vector energy (and consequently the energy of the control signal $u(k))$ will be reduced compared to the standard FxLMS algorithm. Therefore, leakage can also be used to limit the control signal levels. Of course, this leads to an increase in steady state mean squared error compared to the original FxLMS adaptation, i.e. a performance loss.

$$
J_{\text {leaky }}(k)=e(k)^{\mathrm{T}} e(k)+\beta \boldsymbol{w}(k)^{\mathrm{T}} \boldsymbol{w}(k)
$$

Another robustified FxLMS algorithm, which is proposed by [Fraanje et al. (2004)], augments the secondary path with a regularization filter $S_{\text {reg }}(z)$, see equation (5.61).

$$
S_{\text {aug }}(z)=\left[\begin{array}{c}
S(z) \\
S_{\text {reg }}(z)
\end{array}\right], \quad \hat{S}_{\text {aug }}(z)=\left[\begin{array}{c}
\hat{S}(z) \\
S_{\text {reg }}(z)
\end{array}\right]
$$


The resulting SPR condition (5.62) is less strict than equation (5.57) because the last term is non-negative at any frequency $\omega$. In [Fraanje et al. (2007)], several design methods for the regularization filter $S_{\text {reg }}(z)$ are discussed.

$$
\hat{S}^{\mathrm{H}}\left(\mathrm{e}^{j \omega}\right) S\left(\mathrm{e}^{j \omega}\right)+S^{\mathrm{H}}\left(\mathrm{e}^{j \omega}\right) \hat{S}\left(\mathrm{e}^{j \omega}\right)+2 S_{\text {reg }}^{\mathrm{H}}\left(\mathrm{e}^{j \omega}\right) S_{\text {reg }}\left(\mathrm{e}^{j \omega}\right)>0
$$

Recall from section 5.2.4 that the same regularization of the secondary path is used when the frequency-weighted control effort is taken into account in the optimization problem. Therefore, the robustified FxLMS algorithm can also be used to shape the control effort in the frequency domain.

Like the leaky FxLMS algorithm, these robustified algorithms result in performance loss compared to the original FxLMS algorithm (for the nominal plant) and have an increased computational complexity compared to the (leaky) FxLMS algorithm.

\subsubsection{Preventing actuator signal clipping}

Recall from the discussion on the Causal Wiener filter that the (adaptive) feedforward controller should include an inverse of the secondary path outer factor $S_{o}(z)$. For instance, in the preconditioned FxLMS algorithm this inverse outer factor is implemented separately, see figure 5.8.

For most vibration isolation problems, the secondary path is AC-coupled because absolute motion sensors are used, i.e. its DC-gain is zero. As a result, the inverse outer factor $\hat{S}_{o}^{-1}(z)$ has very high low-frequency gain. This high gain, combined with low-frequency noise on the reference sensor, may lead to drift of the actuator signal. Whenever the actuator signal saturates, the weight vector continues to grow out of bounds, which, in practice, leads very rapidly to instability.

Therefore, actuator saturation must be prevented. In principle, this requires a careful selection of the components in the actuator channel, based on the expected excitation level and desired performance level.

However, the adaptation algorithm can also take into account these actuator level considerations. In the previous section, it has already been discussed that the leaky and robustified FxLMS algorithms also restrict the actuator signals, beside improving the robustness to modelling errors. Several variants of the leaky LMS algorithm serve the same purpose, see e.g. [Rossetti et al. (1996); Nascimento and Sayed $(1996,1999)]$.

Another possibility is the noise shaping technique proposed by [Kuo and Yang (1996)], which is illustrated in figure 5.10. The LMS update now uses the modified error signal $\hat{e}(k)$, see equation (5.63).

$$
\hat{e}(k)=d_{e}(k)+S\left[N\left[w^{\mathrm{T}}(k) \psi(k)\right]\right]-\hat{S}\left[N\left[w^{\mathrm{T}}(k) \psi(k)\right]\right]+\hat{S}\left[w^{\mathrm{T}}(k) \psi(k)\right]
$$




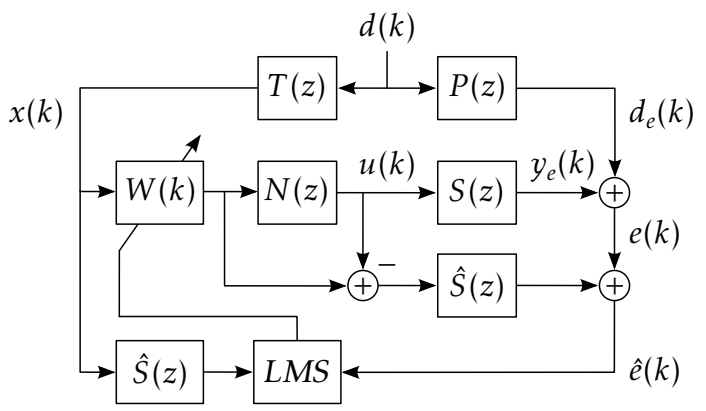

Figure 5.10: FXLMS algorithm with noise shaping filter $N(z)$ [Kuo and Yang (1996)]

Note that the LMS update is unaware of the presence of the noise shaping filter $N(z)$ when the secondary path model is perfect. In this case, the stability and convergence rate results of the original FxLMS algorithm are still valid. Under the assumption that perfect cancellation is possible ${ }^{10}$, the effects of the noise shaping filter on the residual error $E(z)$ and the control signal $U(z)$ are easily derived for the SISO case, see equations (5.64) and (5.65).

$$
\begin{aligned}
& E(z)=(1-N(z)) P(z) D(z)=M(z) P(z) D(z) \\
& U(z)=-N(z) S^{-1}(z) P(z) D(z)
\end{aligned}
$$

Clearly, the noise shaping filter $N(z)$ offers direct control over the spectrum of the control signal. However, the presence of the noise shaping filter has a complementary effect on the error signal, which must also be considered. In order to prevent large low-frequency control signals, the noise shaping filter $N(z)$ should be designed as a high-pass filter with a suitable cut-off frequency and adequate roll-on. On the other hand, the complementary filter $M(z)$ should have adequate roll-off at frequencies above the cut-off frequency. Moreover, the peaking of both filters near the cut-off frequency must not be excessively large as this leads to amplification of the error signal and/or the actuator signal.

The challenge is to design the filters $N(z)$ and $M(z)$ such that they possess the desired properties. Note that this design problem can be thought of as a mixed sensitivity problem in feedback control, because it involves two "sensitivity" functions $N(z)$ and $M(z)$, which are linked by the constraint $N(z)+M(z)=1$. Consequently, any of the available solution procedures for such problems can be used for the filter design, see e.g. [Skogestad and Postlethwaite (2005)]. Lastly, it is pointed out that this noise shaping technique can also be applied in combination with the modified and/or preconditioned FxLMS control structure.

${ }^{10}$ Use $W(z)=-S^{-1}(z) P(z) T^{-1}(z)$ 


\subsubsection{Filter parametrizations}

\section{FIR parametrization}

The most common implementation form for the reference filter $\boldsymbol{F}(z)$ is the tapped delay line, resulting in a Finite Impulse Response (FIR) parametrization for the feedforward controller, see equations (5.66)-(5.68) and figure 5.11. It is recognized that the control signal $u(k)$ is simply a weighted sum of delayed samples of the filtered reference signal $x_{\hat{S}}(k) \equiv \hat{S}[x](k)$.

The FIR parametrization is straightforward to use as it only requires the user to select the number of controller weights $L$. Moreover, the controller is inherently stable as all poles are located at $z=0$.

$$
\begin{aligned}
& \boldsymbol{F}_{\mathrm{FIR}}(z)=\left[\begin{array}{llll}
1 & z^{-1} & \ldots & z^{-L+1}
\end{array}\right]^{\mathrm{T}} \\
& \boldsymbol{\psi}_{\hat{S}}(k)=\boldsymbol{F}_{\mathrm{FIR}}\left[x_{\hat{S}}(k)\right]=\left[\begin{array}{llll}
x_{\hat{S}}(k) & x_{\hat{S}}(k-1) & \ldots & x_{\hat{S}}(k-L+1)
\end{array}\right]^{\mathrm{T}} \\
& u(k)=w^{\mathrm{T}}(k) \psi_{\hat{S}}(k)=\sum_{l=1}^{L} w_{l}(k) x_{\hat{S}}(k-l+1)
\end{aligned}
$$

The controller weights $w(k)$ represent the values of the impulse response of the feedforward controller. Moreover, the impulse response of the controller is finite as it is non-zero for at most $L$ samples.

In order to obtain sufficient vibration isolation performance, enough weights are required to accurately describe the impulse response of the optimal controller, i.e. the Causal Wiener filter. For systems with lightly damped poles and zeros, the latter impulse response may be very long, leading to an excessively large number of weights being required. The available computational capacity of the real-time control system may then be a significant limiting factor for the amount of weights that can be used and, as a consequence, for the overall performance.

It should be noted that the number of weights depends not only on the length of the impulse response of the optimal controller (in seconds), but also on the sample interval $T_{s}$ that is used for the feedforward controller, with smaller sample intervals leading to a larger numbers of required weights.

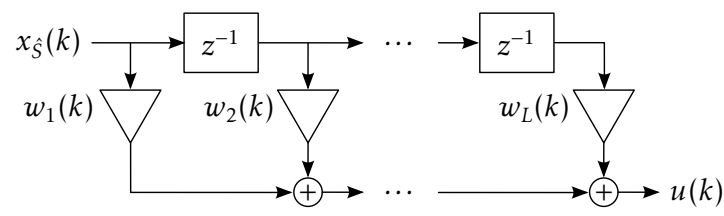

Figure 5.11: FIR parametrization of the feedforward controller 
Lastly, it is pointed out that the FIR feedforward controller can benefit from the presence of a feedback control system. If the feedback control is designed to improve the damping in poorly damped resonance modes, the impulse response can be shortened considerably and the number of weights can therefore be reduced.

\section{IIR parametrizations}

As an alternative to the FIR parametrization, various infinite impulse response (IIR) parametrizations have been proposed in the signal processing literature. For a general overview of adaptive IIR filtering, the interested reader is referred to e.g. [Shynk (1989a); Regalia (1995)]. As the name suggests, such parametrizations are capable of describing infinite impulse responses (with a finite number of parameters). A necessary requirement for this capability is that the parametrization has poles that are not located in $z=0$. Then, two major forms of IIR parametrization can be distinguished: parametrizations with fixed poles and parametrizations with adaptive poles.

The latter parametrizations include the filter pole locations (in some form) as adaptive parameters. Because the pole locations are updated at each iteration, the possibility exists that some poles are updated to unstable locations. Therefore, stability checks have to be build into the update algorithms. These stability checks may be difficult to compute, although they are trivial for the lattice realization or the cascaded and parallel realizations with first and second order filter stages [Shynk (1989b); Regalia (1992)].

However, in contrast to the FIR parametrization, the error signal is no longer a linear function of the IIR parameter vector. As a result, finding the gradient of the error with respect to the parameter vector becomes much more involved. In practice, more computationally complex algorithms such as the Gauss-Newton or recursive least-squares (RLS) methods are required to let the adaptation converge (usually at relatively slow rates), see e.g. [Tzes and Le (1996)] for an application in system identification. Moreover, the cost criterion may exhibit local minima and/or saddle points [Fan et al. (1988); Nayeri and Jenkins (1989)]. Due to these difficulties, adaptive IIR filters with adaptive poles will not be considered further in this thesis.

The IIR parametrization with fixed poles can be considered as being halfway between the FIR structure and a "full-blown" adaptive IIR filter. The specific IIR filter structure with fixed poles that is considered in this thesis is shown in figure 5.12 [Heuberger et al. (2005)]. Its basic structure is a cascaded connection of second order sections (SOS) and first order sections (FOS). 


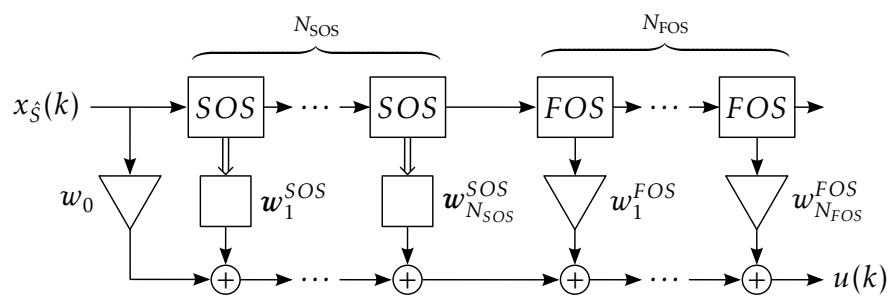

Figure 5.12: IIR filter consisting of $N_{S O S}$ second order sections (SOS) and $N_{F O S}$ first order sections (FOS), with possibly adaptive weights.

The structure of the first and second order sections is shown in figure 5.13. For either section type, the transfer path from $u_{i}^{\mathrm{F} / \mathrm{SOS}}$ to $y_{\mathrm{all}, i}^{\mathrm{F} / \mathrm{SOS}}$ is an all-pass function. This ensures that the power of the filtered reference signal $x_{\hat{S}}(k)$ is transferred to all sections. The interested reader is referred to appendix F.2 for expressions relating the section parameters to the filter poles.

The overall output signal $u(k)$ of the IIR filter is the weighted summation of the states of the second and first order sections, combined with a direct feedthrough term $w_{0}$, see figure 5.12. Note that the weights $w_{i}^{\text {SOS }}$ for the second order sections are 2-element vectors.

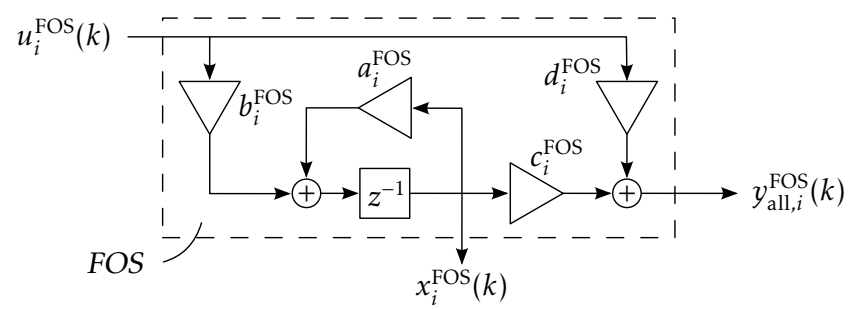

(a) First order section (FOS)

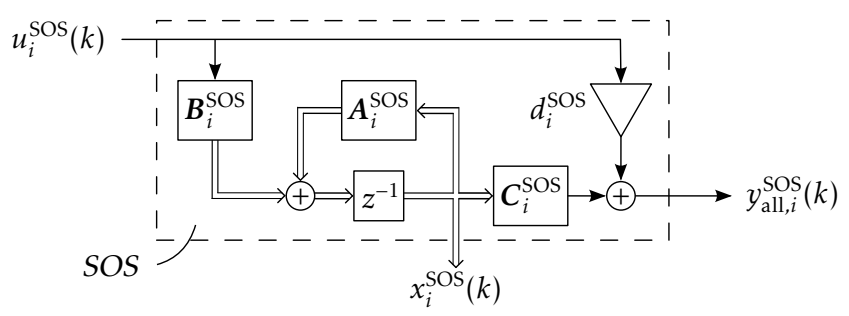

(b) Second order section (SOS)

Figure 5.13: Internal structure of the first and second order sections that are used in the IIR filter with fixed poles. See appendix F.2 for expressions relating the filter parameters to the filter poles. 
The computation of the output signal $u(k)$ is of the same form as the linear regression (5.41), with the states of the sections forming the regression vector. Therefore, this IIR parametrization can be used directly as the regression filter $\boldsymbol{F}(z)$ in the FxLMS algorithm and all the extensions that have been discussed in the preceding sections.

The IIR filter structure in figure 5.12 is a particular realization of so-called orthogonal basis functions for linear systems [Heuberger et al. $(1993,2005)]$. The use of such orthogonal basis functions have been proposed mostly for system identification and modelling [Wahlberg (1994); van den Hof et al. (1995)]. As special cases, these orthogonal basis functions include well-known filter structures such as FIR and Laguerre ${ }^{11}$ [Yuan (2007)].

Note that the IIR filter structure of figure 5.12 can also be thought of as an alternative to the modal expansion of a linear system, which uses parallel first and second order sections (for complex conjugate pole pairs), whereas the IIR filter structure uses a cascaded connection.

This IIR parametrization can be favourable compared to the FIR parametrization when the system being modelled has a very long impulse response. However, the ability of the IIR filter structure of figure 5.12 to model a particular system with relatively few parameters depends on the choice of the pole locations of the filter. For Laguerre filters, a method for finding the optimal pole location is presented in [Oliveira e Silva (1995)]. For general orthogonal basis functions of higher order this requires a numerical search [Heuberger et al. (2005), Ch. 11].

From an engineering point of view, the poles of the IIR filter structure should be close to the actual poles of optimal filter. Therefore, any prior knowledge on the system (from e.g. measured spectral data or system identification) can be incorporated into the controller structure. Recall from the discussion on the Causal Wiener filter in section 5.2.2 that the poles of this filter are determined by the poles and zeros of certain transfer paths in the system. As a result, both information on the system poles and the zeros of the secondary path and tertiary path should be obtained.

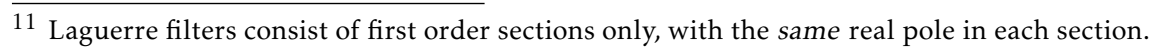




\subsubsection{Computational requirements}

It is customary to use the required number of multiplications as a (rough) indicator for an algorithm's computational complexity. The number of multiplications required for the standard (multiple channel) FxLMS algorithm using an FIR parametrization is given by equation (5.69) [Douglas (1997)]. Here, $N_{r}, N_{u}$ and $N_{e}$ denote the number of reference, actuator and error signals respectively. $L_{\text {FIR }}$ is the number of FIR weights and $M_{\hat{S}}$ is the number of multiplications required for a single channel of the secondary path filter ${ }^{12}$.

$$
\begin{aligned}
& M_{\mathrm{FxLMS}}^{\mathrm{FIR}}=N_{r} N_{u}\left[\left(N_{e}+1\right) L_{\mathrm{FIR}}+N_{e} M_{\hat{S}}\right]+N_{e} \\
& M_{\mathrm{FxLMS}}^{\mathrm{IIR}}=N_{r} N_{u}\left[\left(N_{e}+1\right)\left(L_{\mathrm{IIR}}+M_{I}\right)+N_{e} M_{\hat{S}}\right]+N_{e}
\end{aligned}
$$

The number of multiplications for the IIR parametrization (with fixed poles) is given by equation (5.70), where $L_{\text {IIR }}$ is the number of IIR weights and $M_{I}$ is the number of multiplications required for the computation of the states of the IIR filter. An efficient computation of the states of an IIR filter as shown in figure 5.12 requires less than $5 L_{\text {IIR }}$ multiplications. Therefore, this particular IIR implementation is likely to be more efficient when $6 L_{\text {IIR }}<L_{\text {FIR }}$.

Note that the computational complexity also depends on the secondary path model $\hat{S}(z)$. Therefore, an efficient implementation of this filter is also beneficial. One possibility is to implement the filter as a state-space filter with block-diagonal state matrix, see appendix F.3.

Moreover, for the preconditioned FxLMS algorithm, the term $N_{e} M_{\hat{S}}$ is replaced with $M_{\hat{S}_{o}^{-1}}+N_{e} M_{\hat{S}_{i}}$. Here, $M_{\hat{S}_{o}^{-1}}$ and $M_{\hat{S}_{i}}$ denote the number of multiplications for the inverse outer factor filtering and the inner factor filtering respectively. As the inner factor is generally of lesser order than the original secondary path $\hat{S}(z)$, the computational requirements for systems with multiple error channels are reduced.

For multiple channel systems, the computational complexity can be approximated by $N_{r} N_{u} N_{e} Z$, where $Z$ depends on the (FIR or IIR) parametrization and the implementation of the secondary path filtering. Assuming that the number of error sensors, reference sensors and actuators all equal $N$, it is recognized that the complexity of the multiple channel FxLMS algorithm scales with $N^{3}$, leading to excessively large computational complexity for even moderately sized systems.

A fast version of the multiple channel FxLMS algorithm has been proposed, which makes use of the shift structure inherent to the FIR parametrization [Douglas (1999)]. The resulting algorithm scales with $N^{2}$ instead of $N^{3}$.

\footnotetext{
12 Actually, it is assumed that each channel of the secondary path is evaluated separately. In practice, a MIMO state-space implementation can be more efficient.
} 
Another efficient algorithm for multiple channel systems is the adjoint or filtered error LMS (FeLMS) algorithm, proposed by [Wan (1996)], see also e.g. [Elliott (1998)]. It does not rely on the shift structure in the FIR filter and can therefore also be used with the IIR parametrization. For the FIR parametrization, the required number of multiplications is given by equation (5.71), which only scales with $N^{2}$ for equal numbers of sensors and actuators. For real-time implementations, the FeLMS algorithm requires delays in the adaptation to make the error filtering causal. The convergence rate is reduced due to the presence of these delays. However, this can be partly remedied by applying the preconditioned and modified LMS techniques discussed in section 5.3.2, see e.g. [Berkhoff (2006); Berkhoff and Nijsse (2007)].

$$
M_{\mathrm{FeLMS}}^{\mathrm{FIR}}=N_{u}\left(2 N_{r} L_{\mathrm{FIR}}+N_{e} M_{\hat{S}}\right)+N_{e}
$$

The FeLMS algorithm is not discussed further in this thesis, because the experiments (see chapter 8 ) have been performed on a single channel experimental setup. The interested reader is referred to the previously cited references for details on the FeLMS algorithm.

\subsection{Recapitulation}

In section 5.1, it is discussed first that the active compensation of floor vibrations can indeed be cast into a feedforward problem by using internal model compensation (IMC) of the quaternary path $Q(z)$.

\section{Causal Wiener filter}

The Causal Wiener filter is the causal, stable feedforward controller that minimizes the mean-square error. This Causal Wiener filter is shown to depend on three transfer paths in the system, the primary path $P(z)$, the secondary path $S(z)$ and the tertiary path $T(z)$. Perfect cancellation can not be achieved when the secondary path and/or tertiary path contain delays or unstable zeros that are not cancelled in the primary path.

Moreover, it is shown that the structure of the feedforward problem (i.e. more disturbance sources than actuators, or more sensors than actuators) may give rise to additional performance loss. In practice, due to the presence of measurement noise, such structural problems always occur.

Not surprisingly, the optimal feedforward controller can not compensate for noise on the error sensors that is not correlated with the reference signals. Moreover, it is shown that noise on the reference sensors causes a performance loss, especially at frequencies where the signal-to-noise ratio is small. 
Finally, it has been demonstrated that frequency-dependent error weighing as well as control effort weighing can be easily included into the presented framework.

\section{Adaptive feedforward control}

Because the primary and tertiary paths may be difficult to model/identify in practice, an adaptive control approach is used to approximate the Causal Wiener filter. The adaptive control theory is presented in section 5.3. A linear regression form of the controller is adopted and the instantaneous squared error is minimized. This cost criterion leads to the well-known FxLMS algorithm which obtains a unique global minimum.

Stability requirements for the update law are reviewed, which are especially straightforward (data-independent) for the $\epsilon$-FxNLMS algorithm. Moreover, the preconditioned and modified FxLMS algorithms are presented in section 5.3.2, which improve the convergence rate of the algorithm.

The adaptation algorithm uses a model of the secondary path $S(z)$ to determine the update direction for the controller parameters. It is shown in section 5.3.3 that the adaptation can be stabilized when the absolute phase error of the secondary path model is less than $90^{\circ}$. However, the resulting performance (in terms of mean-squared error and convergence rate) may have deteriorated. Leaky and robustified FxLMS algorithms are reviewed, which increase the robustness to model errors, at the expense of some loss of nominal performance. These algorithms are also useful for restricting the peak actuator level, which may otherwise lead to clipping and instability. For this purpose, a residual noise shaping filter can also be included in the actuator channel.

Two possible filter parametrizations for the regression filter are discussed. The most common FIR parametrization describes the controller in terms of its impulse response coefficients. Although straightforward and very flexible, this parametrization may require a very large number of parameters, leading to real-time implementation issues due to the limited computing capability of the control system. Therefore, a potentially more efficient IIR parametrization with fixed poles is proposed. However, the performance of the IIR filter depends critically on the chosen set of poles, which requires the user to obtain prior knowledge of the system.

Finally, it is discussed that the FxLMS algorithm can be extended to the multiple channel case, but that the computational complexity increases rapidly with increasing system size. A more efficient alternative for multiple channel systems is the FeLMS algorithm, which allows much of the discussed techniques (preconditioning, robustification and IIR filter parametrization) to be carried over without difficulty. 


\section{Final remarks}

In chapter 8 , experimental results obtained on a single channel experiment setup are presented. In these experiments, a combination of the modified and preconditioned FxLMS algorithm is used, with an $\epsilon$-normalized step size. Moreover, a leakage factor, regularization of the secondary path and a noise shaping filter are used to prevent the actuator signal from clipping. Both the FIR and IIR parametrizations of the adaptive feedforward controller have been tested during the experiments.

As a last remark, it is pointed out once more that the adaptive feedforward control presented in this chapter requires accurate models of the quaternary path $Q(z)$ (for internal model compensation) and the secondary path $S(z)$ (for the adaptation). In chapter 7 , a system identification method is discussed that can be used to obtain such accurate models. 


\section{Chapter 6}

\section{Sensors and actuators for vibration isolation systems}

In this chapter the selection of sensors and actuators for an active hard mount vibration isolation system is discussed. We start with a discussion on sensors in section 6.1, which is restricted to absolute motion sensors only. The considerations regarding actuators are discussed in section 6.2 .

\subsection{Absolute motion sensors}

In chapter 4 , it has been discussed that absolute machine motion measurement is preferred over force measurement and relative motion measurement when regarding active damping by feedback control. Only by using absolute motion measurement, skyhook damping of the suspension mode and improved damping of the structural mode can be achieved. Moreover, it has been shown that the absolute motion measurement results in a practically collocated system, resulting in excellent stability margins.

Recall from chapter 5 that the adaptive feedforward controller requires a reference measurement which should be highly correlated with the disturbance. In the hard mount vibration isolation system, the feedforward controller is used to compensate for floor vibrations. Therefore, the absolute floor motion should be measured. Moreover, the adaptive feedforward controller requires an error signal which should be minimized. In this thesis, the absolute machine motion signal that is used for feedback control is also (conveniently) used as the feedforward error signal. Therefore, in this chapter, only absolute motion sensors are considered. 
In section 6.1.1, a generic model for absolute motion sensors is presented. Based on this model, several sensor types are discussed. Then, in section 6.1.2, sensor requirements are derived for hard mount vibration isolation systems. Based on these requirements, the suitability of several sensor models is investigated further in sections 6.1.3-6.1.6.

\subsubsection{Generic model of absolute motion sensors}

Absolute motion sensors can be categorized into accelerometers, absolute velocity sensors and absolute position sensors. The latter are very uncommon except in seismological instruments, and are not considered in this thesis. The common factor in all absolute motion sensors is the use of a suspended seismic mass.

In figure 6.1, a simplified planar model of an absolute motion sensor is shown. The sensor is modelled as a linear-guided seismic mass $m_{s}$, with stiffness $k_{s}$ and damping $d_{s}$, which is mounted on some (rigid) base. It is assumed that the base motion is unaffected by the presence of the sensor, i.e. the base motion $x_{b}$ is considered an independent variable. Note that the base motion $x_{b}$ and the sensor motion $x_{s}$ are expressed in a local coordinate system that coincides with the sensitive axis of the sensor.

The (nonlinear) equation of motion for the seismic mass, in the direction of the sensitive axis, is given by equation (6.1). In vibration isolation applications the relevant amplitudes of motion are usually small. Therefore, the nonlinear differential equation can be linearized around the nominal position $\left(\bar{x}_{s}, \bar{x}_{b}, \bar{\theta}\right)$. After Laplace transformation (assuming zero initial conditions), the linearized differential equation is is expressed by equation (6.2). ${ }^{1}$

$$
\begin{aligned}
m_{s} \ddot{x}_{s}(t)+d_{s} \dot{x}_{s}(t)+k_{s} x_{s}(t) & =d_{s} \dot{x}_{b}(t)+k_{s} x_{b}(t)-m_{s} g \sin (\theta(t)) \\
\left(m_{s} s^{2}+d_{s} s+k_{s}\right) X_{s}(s) & =\left(d_{s} s+k_{s}\right) X_{b}(s)-m_{s} g \cos (\bar{\theta}) \Theta(s)
\end{aligned}
$$

The deflection $\Delta X_{s}(s)$ of the seismic mass with respect to the sensor housing, as a function of the base displacement $X_{b}(s)$ and the change in orientation $\Theta(s)$, is expressed by equation (6.3). Here, $\omega_{s}$ is the sensor's resonance frequency and $\zeta_{s}$ is the damping ratio associated with $\omega_{s}$, see equation (6.4).

$$
\begin{aligned}
& \Delta X_{s}(s)=X_{s}(s)-X_{b}(s)=-\frac{s^{2} X_{b}(s)}{s^{2}+2 \zeta_{s} \omega_{s} s+\omega_{s}^{2}}-\frac{g \cos (\bar{\theta}) \Theta(s)}{s^{2}+2 \zeta_{s} \omega_{s} s+\omega_{s}^{2}} \\
& \omega_{s}=\sqrt{\frac{k_{s}}{m_{s}}}, \quad \zeta_{s}=\frac{d_{s}}{2 \sqrt{k_{s} m_{s}}}
\end{aligned}
$$

1 Note that the variables $X_{s}(s), X_{b}(s)$ and $\Theta(s)$ now represent the (Laplace-transformed) deviations from the nominal position $\left(\bar{x}_{s}, \bar{x}_{b}, \bar{\theta}\right)$. 


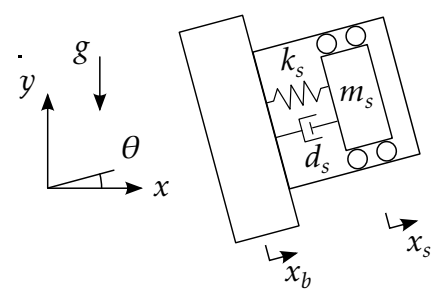

Figure 6.1: Mass-damper-spring model of an absolute sensor in $2 D$ space

\section{Accelerometers}

Ignoring the effect of rotations for now, it is observed from the equation (6.3) that the deflection is a measure for the base acceleration $s^{2} X_{b}(s)=\ddot{X}_{b}(s)$ for frequencies below the resonance frequency $\omega_{s}$. Assuming a displacement sensitivity $S_{a}$, the accelerometer output $V_{a}(s)$ at low frequencies is given by equation (6.5).

$$
V_{a}(s)=S_{a} \Delta X_{s}(s) \approx-\frac{S_{a}}{\omega_{s}^{2}} s^{2} X_{b}(s) \quad|s| \ll \omega_{s}
$$

As the overall acceleration sensitivity is inversely proportional to the squared resonance frequency, there exists a clear trade-off between the useful bandwidth and the sensitivity for any accelerometer.

Various measurement methods can be used to measure the displacement of the seismic mass, i.e. piezoelectric (PE), piezoresistive (PR) or variable capacitance [Harris and Piersol (2002), Ch. 12].

The most common accelerometers are based on the piezoelectric effect. In these sensors, a piezoelectric element acts as the spring element. In most accelerometers, the element is loaded in shear, providing higher sensitivity and lower transverse sensitivity than compression loaded elements [Yurish (1993)]. A charge is generated when the piezoelectric element is stressed (due to the acceleration of the seismic mass). Due to charge leakage, piezoelectric sensors can not measure steady-state acceleration.

In piezoresistive sensors, the resistance of a sensitive element is changed due to strain. These sensors usually employ a (full) Wheatstone bridge configuration to improve sensitivity and reduce e.g. temperature effects, see e.g. [Partridge et al. (2000)].

Variable capacitance sensors measure the variation of capacitance due to the displacement of electrodes mounted on the seismic mass. Usually, a differential measurement is performed, in order to improve the linearity. For a comparison of commercially available variable capacitance sensors, see e.g. [Acar and Shkel (2003)]. 
Variable capacitance sensors are capable of measuring steady-state acceleration. Moreover, these sensors are commonly produced as MEMS-devices, providing a very small sensor package. However, the inherently small seismic mass limits the intrinsic sensitivity of these MEMS-based sensors.

Generally, accelerometers are operated in open loop. However, servo or forcebalance sensors are available, which incorporate a means of actuation (i.e. electrostatic, electromagnetic or piezoelectric) and an internal control circuit. Within the control bandwidth, the control signal required to constrain the displacement of the seismic mass to zero is proportional to the applied base acceleration. Because the amplitude of motion is reduced compared to open loop sensors, servo accelerometers have improved linearity and hysteresis characteristics.

\section{Geophones}

Based on equation (6.3), the displacement velocity transfer function due to base velocity at frequencies above the sensor's resonance frequency is approximately minus one, see equation (6.6). In other words, the displacement velocity has a high-pass response to a base velocity input.

$$
\frac{s \Delta X_{s}(s)}{s X_{b}(s)} \approx-1 \quad|s| \gg \omega_{s}
$$

In absolute velocity sensors, which are commonly called geophones due to their application in geological and seismological applications, the displacement velocity is commonly measured by electromagnetic induction, see equation (6.7). Unlike accelerometers, the velocity sensitivity (within the useful bandwidth) $S_{g}$ is not influenced by the resonance frequency, but is completely determined by the properties of the coil/magnet assembly [Riedesel et al. (1990), App. 1].

$$
V_{\mathrm{g}}(s)=-S_{g} s \Delta X_{s}(s)
$$

In order to obtain a large useful bandwidth, an important challenge in geophone design is to achieve a low resonance frequency in the sensor's sensitive direction, without reducing the other resonance frequencies too much. These spurious frequencies can introduce artificial components into the velocity measurement and therefore limit the useful measurement bandwidth [Ling et al. (1990); Faber and Maxwell (1997)]. In many applications, so-called stretching filters are used to improve the response at low frequencies, see e.g. [Zuo and Nayfeh (2004)]. 


\section{Tilt-to-horizontal coupling}

The second term in equation (6.3) indicates that any absolute motion sensor is also sensitive to variations of the orientation in the gravitational field $\Theta(s)$, see equation (6.8). This is commonly referred to as tilt-to-horizontal coupling. The coupling is maximal for horizontally oriented sensors, i.e. $\bar{\theta}=0$, whereas vertically oriented sensors ideally have zero coupling.

$$
\frac{\Delta X_{s}(s)}{\Theta(s)}=-\frac{g \cos (\bar{\theta})}{s^{2}+2 \zeta_{s} \omega_{s} s+\omega_{s}^{2}}
$$

For accelerometers, it is therefore impossible to distinguish an acceleration input from an orientation change with respect to the gravitational field. In general, the acceleration levels of a supported machine reduce to (nearly) zero for low frequencies, whereas low frequency orientation changes are still possible, especially in soft mounted machines. Hence, any accelerometer will turn into a tilt sensor at low frequencies. This effect needs to be considered when the sensor signal is used for control.

In the previous paragraphs, it was discussed that geophones have a highpass filter response for absolute velocity. However, from equation (6.8) it can be concluded that a geophone has a low-pass response for rotational velocity. Therefore, this effect should be taken into account when the geophone is mounted on a machine that can rotate at low frequencies. ${ }^{2}$ Especially when stretching filters are used, this can result in erroneous measurement signals.

\subsubsection{Sensor requirements}

As discussed at the beginning of section 6.1, in an active hard mount vibration isolation system, motion sensors are required for measuring the floor motion and the machine motion. The floor motion sensor serves as the reference sensor for the feedforward compensation. The machine motion sensor is used as an error sensor in the feedback control as well as in the adaptation of the feedforward parameters.

Moreover, recall from section 3.1 that the control system should achieve three objectives: damping of the suspension modes, damping of relevant structural modes and improvement of the floor vibration transmissibility.

In this section, requirements for the sensors are derived from these control objectives. Although many properties play a role in the selection of motion sensors for vibration isolation purposes (see the list on the next page for some important properties), the discussion is limited to the sensor noise level and bandwidth.

$\overline{2}$ Once more, this effect is most noticeable in soft mounted machines 
- noise level

- bandwidth

- maximum input range

- nonlinearity / hysteresis

- size / weight

- cost

For most motion sensors, the (effective) noise level is expressed as an equivalent input signal, which is commonly indicated by the term referred to input (RTI), see also equation (6.9). The RTI noise level is a function of the actual noise generated by the sensor and its associated electronics ${ }^{3}$ and the sensor's sensitivity. Usually, the noise level is specified as an RMS-value ${ }^{4}$. In this thesis, the noise power spectral density and 1/3-octave RMS-values are used to obtain more insight in the frequency dependency of the sensor noise.

$$
\text { noise } \mathrm{RTI}=\frac{\text { noise, } \text { referred to output }(\mathrm{RTO})}{\text { sensitivity }}
$$

In order to determine the acceptable sensor noise level and sensor bandwidth, an estimate of the floor acceleration level and the machine acceleration level are computed. Based on the VC-curves and measurement data presented in figure 2.2, the floor vibration level is approximated by an extended VC$\mathrm{E}$ (VC- $\mathrm{E}_{\text {ext }}$ ) curve, as shown in figure 6.2 (thin solid line). This vibration level roughly resembles the vibration levels shown in figure 2.2. For reference, the maximum of the measured floor acceleration is repeated here as well (thick solid line).

Given this floor acceleration level, the resulting machine acceleration is estimated by filtering this floor acceleration level with the transmissibility response of the isolation system.

In section 3.1, a reference transmissibility function $T_{\text {ref }}(s)$ has been introduced that represents a state-of-the-art soft mount system having its suspension frequency at $1 \mathrm{~Hz}$ and $70 \%$ skyhook (active) suspension damping. The estimated machine acceleration level for this reference system is also shown in figure 6.2 (dash-dotted line). Moreover, the estimated machine acceleration is estimated for a passive hard mount system having its suspension frequency at $10 \mathrm{~Hz}$ and only $1 \%$ suspension damping (dashed line).

3 These include power supply, signal conditioning electronics and AD converters

4 The specification should include the frequency range that has been used for determining the RMS-value. Unfortunately, this information is not always indicated on the data sheets, which complicates comparison of different sensors. 


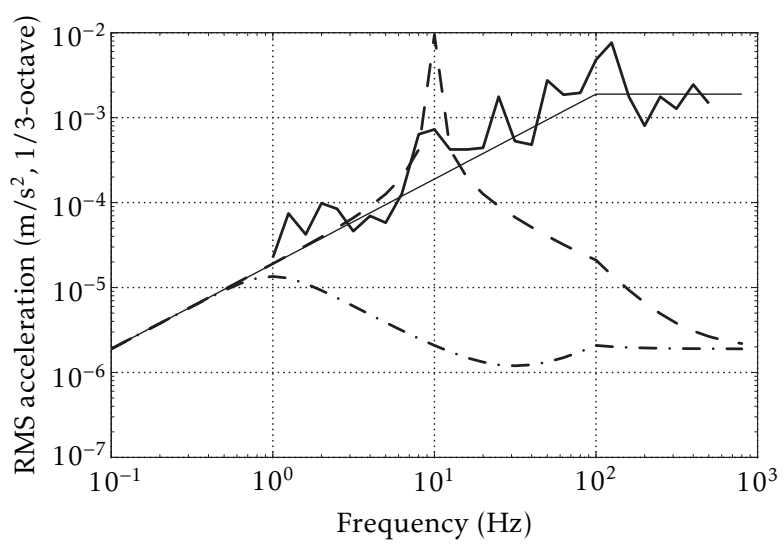

Figure 6.2: Estimated motion levels compared to measured acceleration levels

— : maximum measured floor acceleration levels (see also figure 2.2)

- : VC- $E_{\text {ext }}$ floor acceleration level

- - : estimated (passive) hard mount machine acceleration level

$-\cdot-$ : reference (active) soft mount machine acceleration level

It is clear that the hard mount transmissibility has to be improved in the frequency range from $1 \mathrm{~Hz}$ to at least $500 \mathrm{~Hz}$ (and especially at the poorly damped suspension mode). Therefore, the reference and error sensors are required to have a bandwidth which spans at least this range, say $0.1-1000 \mathrm{~Hz}$.

The data in figure 6.2 is also used to specify the resolution requirements. For one, the reference sensors must be able to resolve the floor acceleration level as indicated by the thin solid line.

The requirements for the error sensor are much more demanding. In order to achieve the soft mount vibration isolation performance, the error sensor's noise level must be lower than this reference level within the active bandwidth of the control system.

\section{Selection of evaluated sensors}

The combined requirement of low noise level and large bandwidth turn out to be very stringent. For example, recall from section 6.1.1 that the bandwidth and sensitivity (and therefore RTI noise) have to be traded-off in the sensor design. Moreover, due to the inductive measurement method, geophones necessarily have a limited bandwidth (in terms of their response to acceleration).

In table 6.1, an overview is presented of several commercially available sensors that have been considered. For most of these sensors, the bandwidth and resolution information could be obtained from the manufacturer's data sheets. 


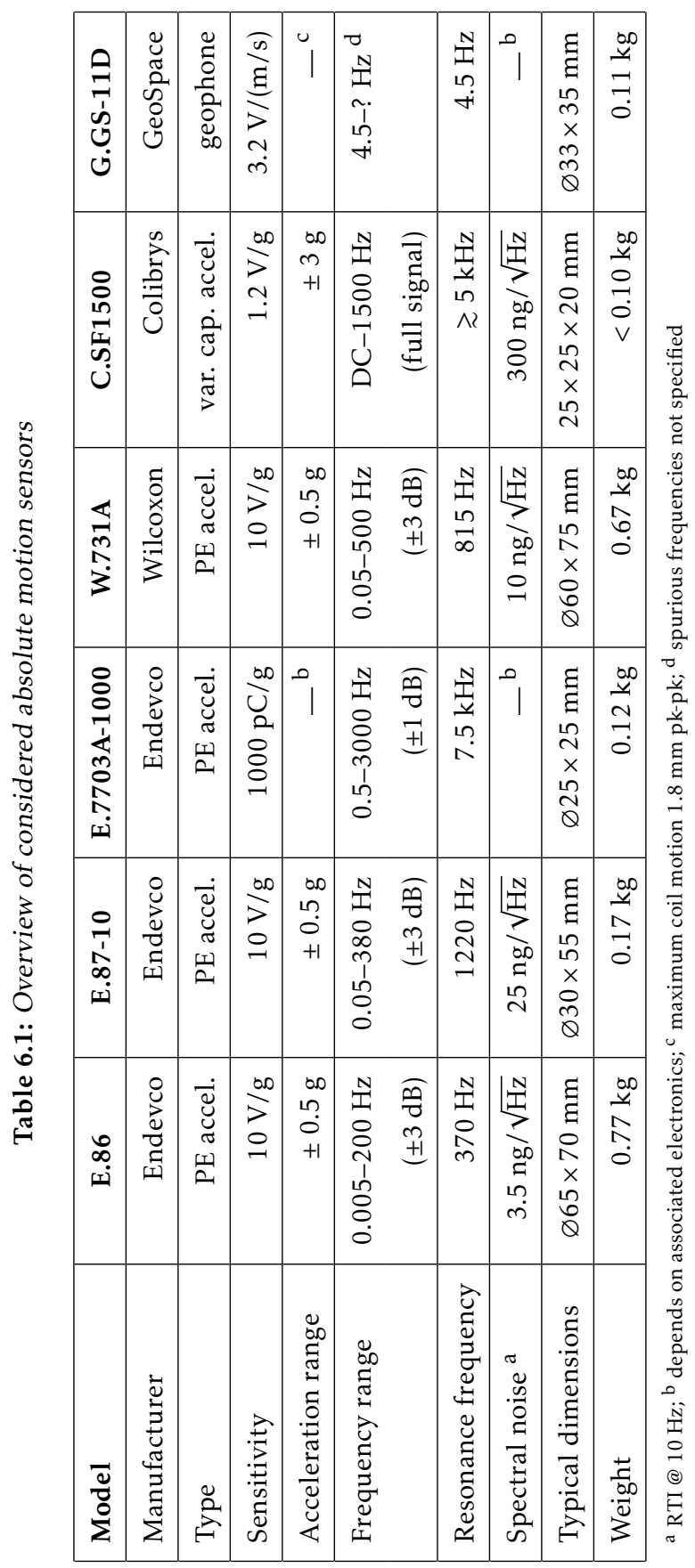


The Endevco 86 and 87-10 models and the Wilcoxon 731A model are seismic accelerometers with excellent noise characteristics. Unfortunately, their useful frequency ranges are rather limited. Moreover, the Endevco 86 and Wilcoxon 731A are prohibitively large and heavy for use in the experimental setup.

The Endevco 7703A-1000 piezoelectric (PE) accelerometer is selected for its high charge sensitivity, combined with a relatively large bandwidth and small size. A charge amplifier is required to convert the charge output to a voltage signal. The noise characteristics of this measurement setup are discussed in section 6.1.4.

The GeoSpace GS-11D geophone is chosen for its intrinsic low noise. It is contained in a fairly small package. Its output signal has to be amplified using a low-noise amplifier circuit, which is discussed in section 6.1.5.

Finally, the Colibrys SiFlex 1500 is considered. This sensor is a variable capacitance, MEMS-based, force-balance accelerometer.

\subsubsection{Determining the noise level of seismic sensors}

Measuring the noise level of motion sensors with seismic sensitivity is not straightforward as the environmental motion levels can be much larger than the sensor's noise level. In some occasions, it is possible to determine the noise level by locking the seismic mass. Another method uses the measurements of two sensors mounted closely together. The noise levels can then be estimated using the coherence between the measured signals [Holcomb (1989); Barzilai et al. (1998)].

The latter estimation method is applied in the following sections to determine the noise level of the Endevco 7703A-1000 and GeoSpace GS-11D sensors. The method is illustrated by the model of figure 6.3. A common motion signal $x(t)$ is observed by two sensors. The sensors are modelled as LTI systems $\left(H_{1}, H_{2}\right)$ and the noise contributions $\left(n_{1}(t), n_{2}(t)\right)$ of each sensor are referred to input. The measurement signals are denoted by $y_{1}(t)$ and $y_{2}(t)$.

It is assumed that the noise signals are uncorrelated and each noise signal is also uncorrelated with the motion signal $x(t)$. Using these assumptions, the power spectral densities and the cross-spectral density of the measurement signals are given by equations (6.10)-(6.12).

$$
\begin{aligned}
& P_{y_{1} y_{1}}(\omega)=\left|H_{1}(j \omega)\right|^{2}\left(P_{x x}(\omega)+P_{n_{1} n_{1}}(\omega)\right) \\
& P_{y_{2} y_{2}}(\omega)=\left|H_{2}(j \omega)\right|^{2}\left(P_{x x}(\omega)+P_{n_{2} n_{2}}(\omega)\right) \\
& C_{y_{1} y_{2}}(j \omega)=H_{2}^{\mathrm{H}}(j \omega) H_{1}(j \omega) P_{x x}(\omega)
\end{aligned}
$$




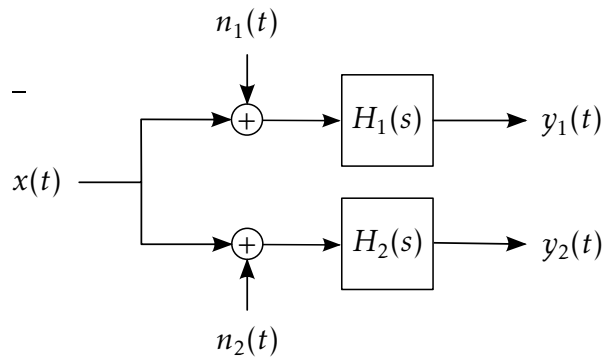

Figure 6.3: Block diagram of the model used for determining the noise level of seismic sensors

Moreover, the magnitude-squared coherence between the measurement signals $\gamma_{y_{1} y_{2}}^{2}(\omega)$ is defined by equation (6.13).

$$
\gamma_{y_{1} y_{2}}^{2}(\omega)=\frac{\left|C_{y_{1} y_{2}}(j \omega)\right|^{2}}{P_{y_{1} y_{1}}(\omega) P_{y_{2} y_{2}}(\omega)}
$$

After some rearranging, the alternative expression (6.14) is found for the noise power spectral density $P_{n_{1} n_{1}}$. A similar expression can be found for $P_{n_{2} n_{2}}$.

$$
P_{n_{1} n_{1}}(\omega)=\frac{P_{y_{1} y_{1}}(\omega)}{\left|H_{1}(j \omega)\right|^{2}}\left(1-\gamma_{y_{1} y_{2}}(\omega) \frac{\left|H_{1}(j \omega)\right|}{\left|H_{2}(j \omega)\right|} \sqrt{\frac{P_{y_{2} y_{2}}(\omega)}{P_{y_{1} y_{1}}(\omega)}}\right)
$$

When the noise spectra $P_{n_{1} n_{1}}$ and $P_{n_{2} n_{2}}$ are approximately equal and the sensor responses $H_{1}(s)$ and $H_{2}(s)$ are also approximately equal, the noise spectral density can be approximated by equation (6.15).

$$
P_{n_{1} n_{1}}(\omega) \approx \frac{P_{y_{1} y_{1}}(\omega)}{\left|H_{1}(j \omega)\right|^{2}}\left(1-\gamma_{y_{1} y_{2}}(\omega)\right)
$$

\subsubsection{Noise level of the Endevco 7703A-1000}

Before applying the method of the previous section to the data collected from the measurement setup, a noise model for piezoelectric accelerometers is discussed.

As mentioned previously, the charge output of the piezoelectric accelerometer has to be converted to a voltage signal. A charge amplifier is preferred for this purpose, because a charge amplifier's gain is independent of the sensor and cable capacitances. 


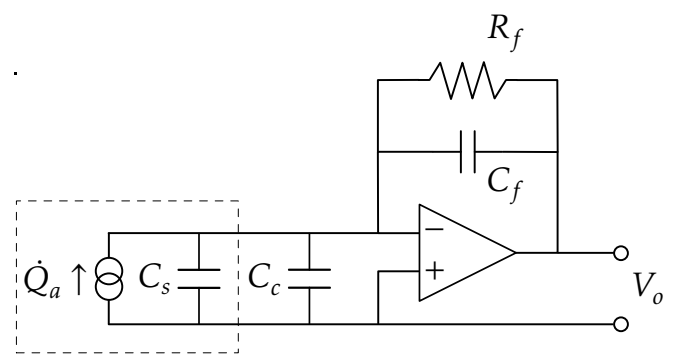

Figure 6.4: Circuit model of a piezoelectric accelerometer (dashed box) connected to a charge amplifier through a cable with capacitance $C_{c}$.

Figure 6.4 shows a diagram of an accelerometer which is connected to a rudimentary charge amplifier. The accelerometer is modelled as a current source $\dot{Q}_{a}$ parallel to a capacitance $C_{s}$. A parasitic cable capacitance $C_{c}$ is also included.

Assuming an ideal operational amplifier (opamp) and infinite input impedance of the volt meter, the feedback resistor $R_{f}$ and the feedback capacitance $C_{f}$ determine the charge-to-voltage frequency response of this circuit, as indicated by equation (6.16).

$$
\frac{V_{o}(s)}{Q_{a}(s)}=-\frac{s R_{f}}{1+s R_{f} C_{f}}
$$

The generated charge $Q_{a}(t)$ due to a base acceleration is given by equation (6.17), where $S_{c}$ is the charge sensitivity of the sensor. This expression is a valid approximation at frequencies below the sensor's resonance frequency ${ }^{5}$.

$$
Q_{a}(t)=S_{c} \ddot{x}_{b}(t)
$$

In order to model the noise characteristics of this circuit, all relevant noise sources need to be identified and taken into account. Noise sources that occur in the piezoelectric sensor itself are thermo-mechanical ${ }^{6}$ and thermoelectrical noise [Gabrielson (1993, 1995); Levinzon $(2004,2005)]$. The cable and the components of the charge amplifier are potential noise sources as well.

To model the thermo-mechanical noise, a force $F_{n}$ can be included in the massdamper-spring sensor model of figure 6.1, parallel to the spring $k$ and damper $d$. The power spectral density $P_{F_{n} F_{n}}$ of $F_{n}$ is independent of frequency and given by equation (6.18). Here, $d_{s}$ is the damping constant $(\mathrm{N} /(\mathrm{m} / \mathrm{s})), T$ is the absolute temperature $(\mathrm{K})$ and $k_{B}$ is Boltzmann's constant $\left(1.38 \cdot 10^{-23} \mathrm{~J} / \mathrm{K}\right)$.

$$
P_{F_{n} F_{n}}=4 k_{B} T d_{s} \quad\left[\mathrm{~N}^{2} / \mathrm{Hz}\right]
$$

5 This is usually the frequency range of interest

6 This is sometimes called Brownian motion 
At frequencies below the sensor's resonance frequency, the thermo-mechanical noise power spectral density is then equivalent to a base acceleration PSD as indicated by equation (6.19).

$$
P_{\ddot{x}_{b} \ddot{x}_{b}}=\frac{P_{F_{n} F_{n}}}{m^{2}}=4 k_{B} T \frac{2 \zeta_{s} \omega_{s}}{m} \quad\left[\left(\mathrm{~m} / \mathrm{s}^{2}\right)^{2} / \mathrm{Hz}\right]
$$

The electrical equivalent of the thermo-mechanical noise is the thermo-electrical noise or Johnson-Nyquist noise [Nyquist (1928)]. For any resistor having a resistance $R$, the voltage noise spectral density is given by the well-known equation (6.20). Actually, this expression is valid for any passive electrical component that is not lossless ${ }^{7}$.

$$
P_{R R}=4 k_{B} T R \quad\left[\mathrm{~V}^{2} / \mathrm{Hz}\right]
$$

The losses in practical capacitive elements are described by the (dielectric) loss factor $\eta$. For piezoelectric materials commonly used in accelerometers (lead zirconate titanate or PZT), the loss factor depends on many factors e.g. composition, fabrication methods, frequency and temperature. However, in [Levinzon (2004)] a nearly constant value of 0.015 is reported, which also seems to correspond well to commercially available materials, see e.g. [Noliac website (WWW)].

Then, the lossy characteristics of the capacitor can be modelled by including an equivalent, frequency dependent, resistor $R_{p}$ in parallel to the capacitor $C_{s}$, see equation (6.21) [Scaife (1998), Ch. 6; Levinzon (2004)].

$$
R_{p}(\omega)=\frac{1}{\eta \omega C_{s}}
$$

With this equivalent resistor, the voltage fluctuations as expressed by equation (6.20) result in the current spectral density of equation (6.22). Finally, the equivalent acceleration PSD of the thermo-electrical noise is given by equation (6.23).

$$
\begin{array}{rlrl}
P_{\dot{Q}_{a} \dot{Q}_{a}} & =\frac{4 k_{B} T}{R_{p}}=4 k_{B} T \eta \omega C_{s} & & {\left[\mathrm{~A}^{2} / \mathrm{Hz}\right]} \\
P_{\ddot{x}_{b} \ddot{x}_{b}}=4 k_{B} T \frac{\eta C_{s}}{S_{c}^{2} \omega} & & {\left[\left(\mathrm{m} / \mathrm{s}^{2}\right)^{2} / \mathrm{Hz}\right]}
\end{array}
$$

Although the cable capacitance $C_{c}$ does not influence the frequency response of the measurement setup, the dielectric loss in the cable will cause thermoelectrical noise. However, when the sensor cable is kept short, the total capacitance of the cable is generally much smaller than $C_{s}$. The noise contribution can then be neglected.

7 A component is "lossy" when the real part of its impedance is positive 
Another noise source in cables is called the triboelectric effect. Charge variations are generated by friction between the various layers in the cable, when the cable is bent or when it vibrates. By using specially designed low-noise cables and clamping the cable, the triboelectric effect can be reduced.

Finally, the components of the charge amplifier generate noise as well. In the measurement setup, a Brüel \& Kjær Nexus 2692 conditioning amplifier is used. This charge amplifier incorporates several amplifier stages as well as high-pass and low-pass filters. Unfortunately, the circuit layout and the component properties of this amplifier are unknown. Therefore, it is impossible to model the noise characteristics of the charge amplifier. Instead, the noise level is measured directly.

\section{Noise measurements}

Measurement data is collected from two horizontally oriented accelerometers, which are mounted on opposite faces of a solid aluminium block (approx. $30 \times 30 \times 30 \mathrm{~mm}$ ), which is fixed to the laboratory table. The accelerometers are connected to a Brüel \& Kjær Nexus 2692 charge amplifier with an overall sensitivity $S_{o}$ of $3.16 \mathrm{~V} /\left(\mathrm{m} / \mathrm{s}^{2}\right)$. The cut-off frequencies of the built-in highpass and low-pass filters are set to $0.1 \mathrm{~Hz}$ and $1 \mathrm{kHz}$ respectively. Both filters are second-order filters. A photograph of the setup is shown in figure 6.5.

The data is collected during 870 seconds, using a dSPACE DS1005 real-time platform equipped with a DS2004 ADC-board (16 channels, 16-bit, $\pm 5 \mathrm{~V}$ full-scale input range, $3.2 \mathrm{kHz}$ sampling rate). Power and cross spectral densities are estimated using Welch's method [Welch (1967)], with sections of $2^{17}$ FFT points $(40.96 \mathrm{sec})$, each with $50 \%$ overlap and filtered by a Hanning window.

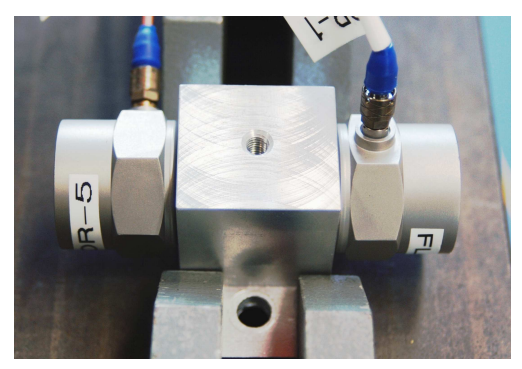

Figure 6.5: Noise measurement setup with two Endevco 7703A-1000 accelerometers, mounted on a solid aluminium block 
The ADC channels have a signal-to-noise ratio (SNR) of $83 \mathrm{~dB}$ for a $\pm 5 \mathrm{~V}$ input $^{8}$, resulting in a $250 \mu \mathrm{V}$ RMS noise level. This noise level has been further reduced by using the burst conversion capabilities of the ADC board. The $\mathrm{ADC}$ channels can perform a consecutive series of $\mathrm{AD}$-conversions at a very high conversion rate (up to $1.25 \mathrm{MHz}$ ), called a burst. As a result, the white noise in the signal is distributed over a much larger frequency range. Then, the signal is low-pass filtered and sampled down to the desired sample frequency, thereby reducing the noise content of the signal.

For the noise measurements, 100 burst conversions at $1 \mathrm{MHz}$ are used, combined with an averaging operation, which serves as the low-pass filter. As a result, the ADC noise level is reduced to approximately $40 \mu \mathrm{V}$ RMS.

The measurement and analysis results are shown in figure 6.6. The total equivalent acceleration PSD is marked by $a$. It is calculated from the measured PSDs and coherence function, according to equation (6.15). The charge amplifier noise has been measured separately, marked by $b$. The ADC noise level (converted to equivalent acceleration) is plotted as well, marked by $c$.

Finally, the thermo-electrical noise model of equation (6.23) is evaluated (dashdotted line), using the following parameter values: $T=300 \mathrm{~K}, \eta=0.02$, $C_{s}=5900 \mathrm{pF}, S_{c}=98 \mathrm{pC} /\left(\mathrm{m} / \mathrm{s}^{2}\right)$. The capacitance $C_{s}$ is taken as the combined capacitance of the sensor $(5600 \mathrm{pF})$ and the cable $(300 \mathrm{pF})$.

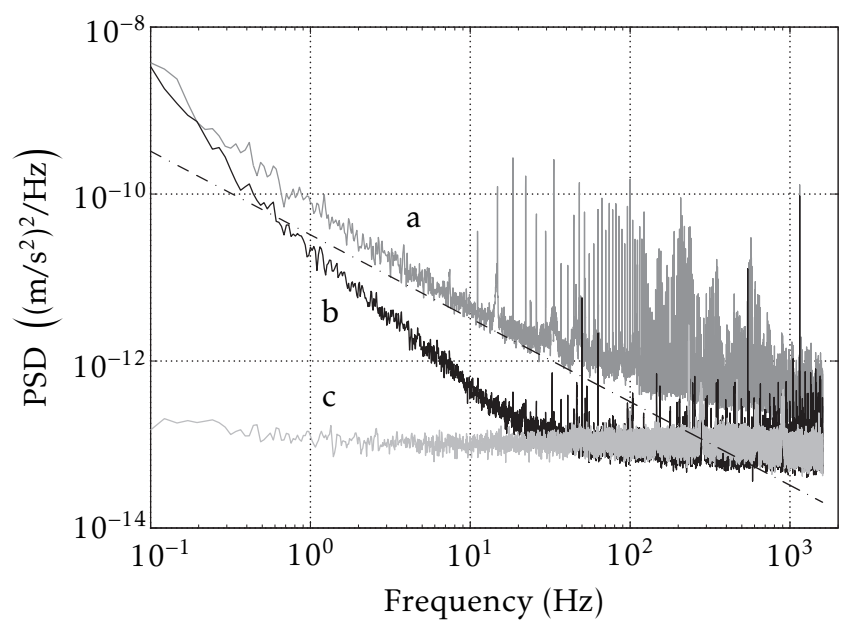

Figure 6.6: Equivalent noise level of the Endevco 7703A-1000 accelerometer

a: Total equivalent noise level

$b$ : Equivalent charge amplifier noise level

c: Equivalent ADC noise level

-.-: Thermo-electrical noise model (eqn. (6.23)),

with $T=300 \mathrm{~K}, \eta=0.02, C_{s}=5900 \mathrm{pF}, S_{c}=98 \mathrm{pC} /\left(\mathrm{m} / \mathrm{s}^{2}\right)$

$8 \pm 5 \mathrm{~V}, 10 \mathrm{kHz}$ sine wave input, $160 \mathrm{kHz}$ sampling rate 
Using $m=0.060 \mathrm{~kg}, \zeta_{s}=0.01$ and $\omega_{s}=2 \pi \cdot 7.5 \cdot 10^{3} \mathrm{rad} / \mathrm{s}$, the thermo-mechanical noise model of equation (6.19) predicts an equivalent acceleration PSD of $2.6 \cdot 10^{-16}\left(\mathrm{~m} / \mathrm{s}^{2}\right)^{2} / \mathrm{Hz}$, which is clearly negligible compared to the other noise sources.

Apparently, the noise level of the Endevco 7703A-1000 is governed by thermoelectrical noise of the sensor itself in the middle frequency range centred on $10 \mathrm{~Hz}$. Below $1 \mathrm{~Hz}$, the $1 / f$-noise of the charge amplifier becomes significant. At high frequencies, many spectral lines occur. These are actually mostly higher harmonics of a $3.7 \mathrm{~Hz}$ base frequency, indicating some nonlinear system behaviour. This nonlinear behaviour appears to originate from the Nexus amplifier, but has not been investigated further.

The ADC noise only becomes important at the far end of the frequency range. This clearly shows the usefulness of the burst conversion functionality of the DS2004 ADC-board. Without it, the equivalent acceleration PSD would become approximately $4 \cdot 10^{-12}\left(\mathrm{~m} / \mathrm{s}^{2}\right)^{2} / \mathrm{Hz}$. Then, the ADC noise would significantly contribute to the noise spectrum at frequencies above $10 \mathrm{~Hz}$.

However, the total conversion time is $100 \mu \mathrm{s}$, which amounts to roughly $30 \%$ of the sampling period. This is acceptable for these measurements, but when the feedforward control algorithms of the previous chapter have to be implemented as well, it is most likely not possible to use this many burst conversions.

\subsubsection{Noise level of the GeoSpace GS-11D}

The GeoSpace GS-11D is available for different orientations, with different coil resistances and different resonance frequencies. In this thesis, the version meant for horizontal operation is tested, with a $380 \Omega$ coil resistance and a $34 \%$ damped resonance frequency at $4.5 \mathrm{~Hz}$. It's sensitivity $S_{g}$ is $32 \mathrm{~V} /(\mathrm{m} / \mathrm{s})$.

The small velocity levels ${ }^{9}$ make additional signal amplification necessary. The amplification circuit is presented in the following section. Moreover, the results of a noise analysis for this circuit and the geophone are presented.

\section{Noise model}

Figure 6.7 shows the three-stage amplifier circuit that was used. The geophone is modelled in the electrical domain as a voltage source $V_{g}$ in series with the coil resistance $R_{c}$. The OP27 opamps serve as input and output buffers, thus eliminating the sensitivity of the amplifier stage to source and load impedances. The OP37 opamp is placed in an inverting gain stage, combined with a first-order low-pass filter.

9 A VC-E input level corresponds to approx. $15 \mu \mathrm{m} / \mathrm{s}$ RMS $(0-1 \mathrm{kHz})$ 


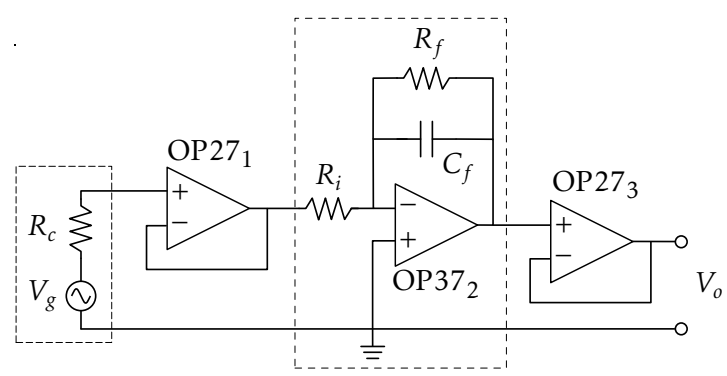

Figure 6.7: Geophone electrical model (dashed,left) and amplifier circuit (input buffer, amplifier stage (dashed, middle) and output buffer)

The geophone response to acceleration $H_{g}(s)$ is described by the transfer function shown in equation (6.24). Here, $S_{g}$ is the geophone sensitivity $(\mathrm{V} /(\mathrm{m} / \mathrm{s}))$, $\zeta_{s}$ is the damping ratio (-) of the geophone resonance frequency $\omega_{s}(\mathrm{rad} / \mathrm{s})$.

$$
H_{g}(s) \equiv \frac{V_{g}(s)}{s^{2} X_{b}(s)}=\frac{S_{g} s}{s^{2}+2 \zeta_{s} \omega_{s} s+\omega_{s}^{2}}
$$

The Analog Devices OP27/37 opamps have been chosen for their low voltage noise. The OP27 opamp was also suggested for the pre-amplifier stage for a comparable geophone in a study by [Riedesel et al. (1990)]. The OP37 is an improved design for large gains $(>5)$. Assuming an ideal opamp, the (dynamic) inverting gain $G_{I}(s)$ of the amplifier stage is given by equation (6.25). The passive components in the amplifier stage are chosen such that $G_{I}(s)$ has a DC gain of -100 and a single pole at $1.9 \mathrm{kHz}$.

$$
G_{I}(s)=-\frac{R_{f}}{R_{i}} \frac{1}{1+s C_{f} R_{f}}
$$

Using basic circuit analysis techniques [Texas Instruments (2007)], the contribution of each noise source is evaluated. The following noise sources are identified in the geophone and the amplifier circuit:

- thermo-mechanical noise (Brownian motion of the seismic mass)

- thermo-electrical noise in $R_{c}, R_{i}$ and $R_{f}$

- dielectric loss in $C_{f}$

- noise in the opamps OP27/OP37

Equations for the thermo-mechanical noise, the thermo-electrical noise and the dielectric loss have already been presented in section 6.1.4, see equations (6.18)-(6.22). 


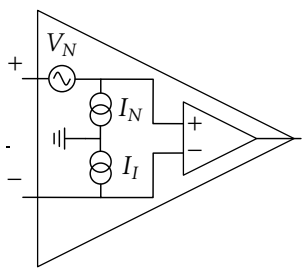

Figure 6.8: Equivalent noise model of an operational amplifier

The noise in opamps is commonly modelled as a current noise source $I_{I}$ at the inverting input of an ideal opamp and a current noise source $I_{N}$ and a voltage noise source $V_{N}$ at the non-inverting input of the ideal opamp, see figure 6.8. It is usually easier to compute the contribution of each noise source to the output voltage PSD $P_{V_{o} V_{o}}(\omega)$, and consequently convert this output PSD back to an equivalent acceleration PSD, as indicated in equation (6.26). The details of the analysis are presented in appendix D.

$$
P_{\ddot{x}_{b} \ddot{x}_{b}}(\omega)=\frac{P_{V_{o} V_{o}}(\omega)}{\left|G_{I}(j \omega)\right|^{2}\left|H_{g}(j \omega)\right|^{2}}
$$

Figure 6.9 shows the predicted equivalent acceleration PSDs. The most dominant noise sources are the voltage noise of opamp $1\left(V_{N_{1}}\right)$ and the voltage noise of opamp $2\left(V_{N_{2}}\right)$, see figure 6.7 for the numbering of the opamps. At the geophone's resonance frequency, the thermo-mechanical noise and the current noise of the first opamp $\left(I_{N_{1}}\right)$ become the most dominant contributions.

\section{Noise measurements}

The noise measurement experiment described earlier in section 6.1.4 is repeated with two geophones. The measurement and analysis results are shown in figure 6.10.

The total equivalent acceleration PSD is marked by $a$. It is calculated from the measured PSDs and coherence function, according to equation (6.15). The ADC noise level (converted to an equivalent acceleration) is plotted as well, marked by $b$. For reference, the predicted noise level of figure 6.9 is shown as well (dash-dotted). This noise prediction is valid for typical opamp noise levels. Another noise prediction is shown (dotted), which is based on maximum opamp noise levels (see also appendix D.2).

It is obvious from this figure that the geophone measurement is approximately 100 times noisier (in power) than predicted (for typical opamp noise values). By comparing the dotted line, it is concluded that this difference is not caused by the (possible) use of worse than typical components. 


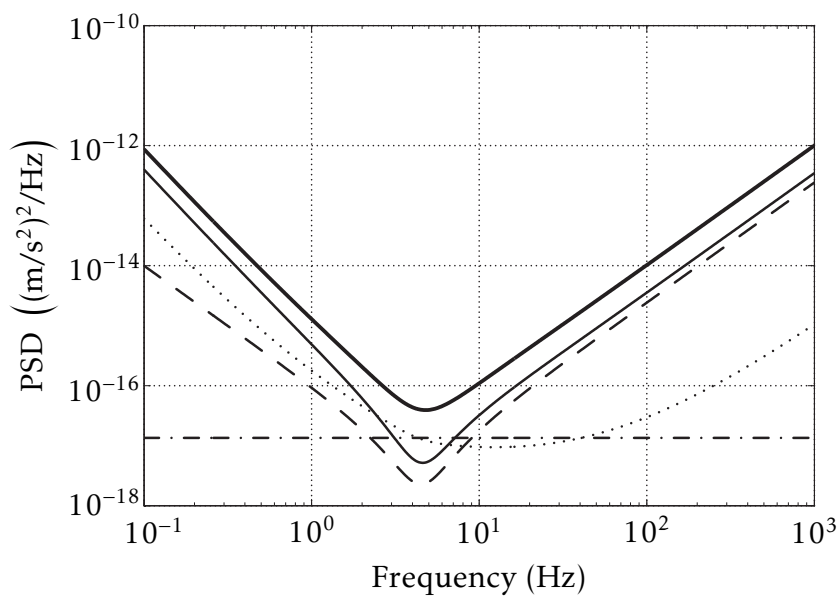

Figure 6.9: Equivalent acceleration PSD of the dominant noise sources in the geophone circuit of figure 6.7, for typical opamp noise levels.

- : Total equivalent acceleration

$-\cdot-$ : Thermo-mechanical noise

- - : Thermo-electrical noise in $R_{c}$

$\cdots$ : Current noise $I_{N_{1}}$

- : Voltage noise $V_{N_{1}}$ and $V_{N_{2}}\left(V_{N_{2}}\right.$ curve is overlaid on $V_{N_{1}}$ curve)

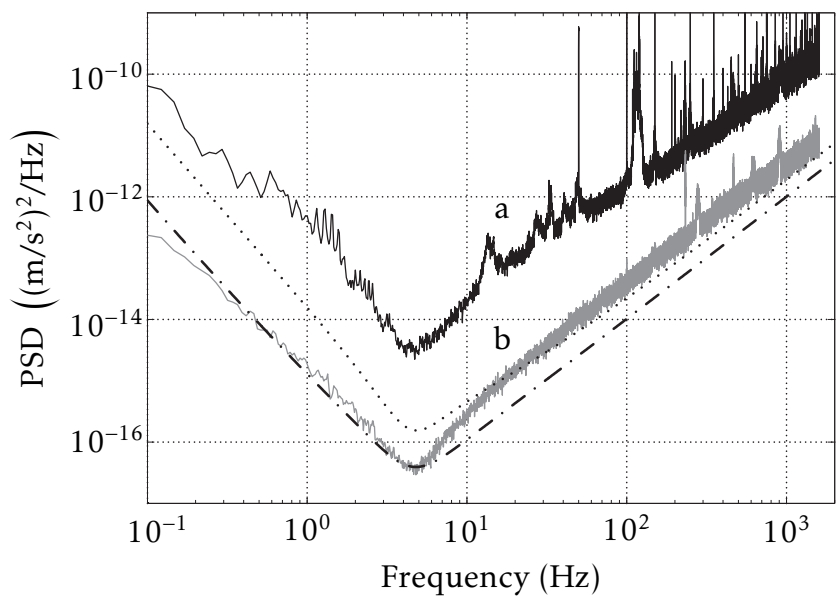

Figure 6.10: Equivalent noise level of the GeoSpace GS-11D geophone a: Total equivalent noise level

$b$ : Equivalent ADC noise level

-.- : Predicted noise level (typical opamp noise levels)

... : Predicted noise level (maximum opamp noise levels) 
The noise level is also not dominated by the ADC noise (see curve $b$ ). Once more, the usefulness of the burst conversions is demonstrated here, as the ADC noise level would increase by a factor 40 without the use of burst conversions.

The origin of the increased noise level is thought to lie in electromagnetic interference. The amplifier circuitry has been implemented on a prototype board, which allows easy replacement of components. However, this may have greatly increased the susceptibility to interference noise sources. For one, the $50 \mathrm{~Hz}$ power supply frequency and several higher harmonics are clearly visible in the noise spectral density.

Another disconcerting feature is the increased noise level in the frequency range 100-130 Hz. Apparently, the frequency response of the tested geophones is dissimilar in this range. This may be caused by spurious resonances of their seismic masses.

\subsubsection{Comparison of evaluated sensors}

In order to assess the suitability of the various sensors, their noise levels are compared with the estimated motion levels that have been presented in section 6.1.2 (figure 6.2).

In figure 6.11, these motion levels are compared to the measured noise levels of the Endevco 7703A and the GeoSpace GS-11D. For this purpose, the estimated noise PSDs have been converted to RMS acceleration in one-third octave bandwidth.

In figure 6.12, the same comparison is made for the noise level of the remaining sensors, see also table 6.1. Their equivalent noise PSDs have been extracted from their respective data sheets and converted to RMS acceleration in one-third octave bandwidth.

It is no surprise that the seismic accelerometer models (Endevco 86, 87-10 and Wilcoxon 731A) have the lowest noise level. However, the price to pay is their limited useful frequency range.

The performance of the Colibrys SiFlex 1500 at low frequencies is remarkable due to the force-balance principle, which eliminates the otherwise typical $1 / f$-noise. At higher frequencies, the relatively small sensitivity results in an increased noise level compared to the other sensors.

The Endevco 7703A-1000 has relatively poor low frequency performance, but has the lowest noise level at high frequencies of the "full bandwidth sensors" (Endevco 7703A-1000, GeoSpace GS-11D and Colibrys SF1500).

The GeoSpace GS-11D geophone performs as good as the seismic accelerometers near its resonance frequency. However, at very low frequencies $(f \lesssim 0.1$ $\mathrm{Hz}$ ) and high frequencies $(f>100 \mathrm{~Hz})$, the noise level increases rapidly. This is inherent to the inductive measurement method. 


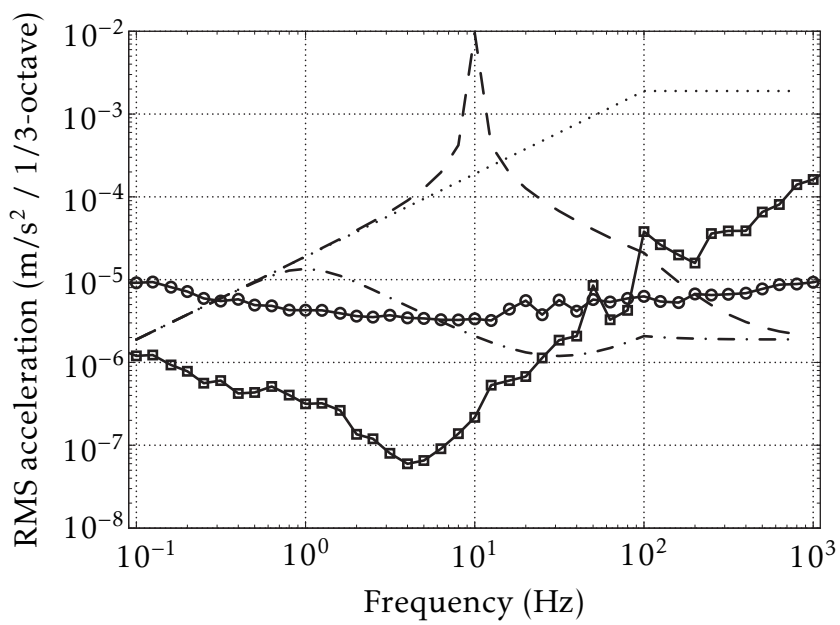

Figure 6.11: Sensor noise levels (RTI) and estimated acceleration levels (1) $\cdots:$ : $V C-E_{\text {ext }}$ floor acceleration level

- - : estimated hard mount machine acceleration level

$-\cdot-$ : estimated soft mount machine acceleration level

$\checkmark$ : Endevco 7703A-1000 equivalent noise level

$\square$ : GeoSpace GS-11D equivalent noise level

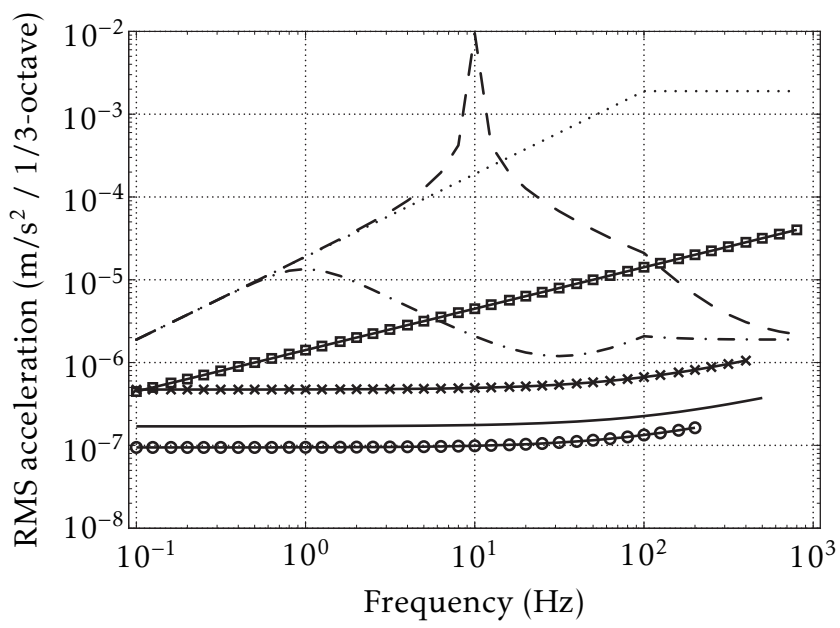

Figure 6.12: Sensor noise levels (RTI) and estimated acceleration levels (2) ... : VC-E $E_{\text {ext }}$ floor acceleration level

- - : estimated hard mount machine acceleration level

-.- : estimated soft mount machine acceleration level

$\square$ : Colibrys SiFlex 1500 equivalent noise level

* : Endevco 87-10 equivalent noise level

— : Wilcoxon $731 \mathrm{~A}$ equivalent noise level

$\odot$ : Endevco 86 equivalent noise level 
By comparing the noise levels with the expected motion levels, it is observed that all sensors are capable of resolving the expected floor motion, except for the Endevco 7703A-1000 for $f \lesssim 0.5 \mathrm{~Hz}$.

With respect to the expected machine motion level, the situation is quite different. Recall from section 6.1.2 that the control objective is to reduce the floor vibration isolation performance of the hard mount system (dashed lines in figures 6.11 and 6.12) to a level comparable to a soft mount system (dashdotted lines). For this purpose, the error sensor(s) should be able to resolve the soft mount motion level.

Only the seismic accelerometers can resolve this motion level over their frequency range. The "full bandwidth sensors" fail to resolve this motion level above $5 \mathrm{~Hz}$ (Endevco 7703A, Colibrys SF1500) and $20 \mathrm{~Hz}$ (GeoSpace GS-11D).

\subsubsection{Recapitulation and final remarks}

A general model for absolute motion sensors has been presented. Using this model, it has been shown that all such sensors suffer from horizontal-to-tilt coupling. At low frequencies, and especially for soft mount systems, this effect on the measurement signal can dominate over the intended signal and should therefore not be ignored.

Based on expected motion levels for the floor and the machine, requirements for the sensors in terms of bandwidth and resolution have been stated. In order to evaluate sensor performance in terms of these requirements, the signal conditioning and noise modelling of several commercially available sensors has been discussed. Moreover, the noise level of two sensor types has been determined experimentally.

It has been shown that many sensors are capable of resolving the expected floor motion levels. On the other hand, none of the sensors is capable of resolving the target machine acceleration level over the entire frequency range. Therefore, it is expected that the floor vibration isolation performance of hard mount systems will be limited by the sensor noise level.

The experimental setup will be equipped with the Endevco 7703A-1000 accelerometers as these sensors offer the best combination of bandwidth, noise level and size. For the experiments, it is attempted to circumvent the problem regarding the sensor noise levels by increasing the excitation level. 


\subsection{Actuators}

In this thesis, two types of actuators have been considered for use in active hard mount vibration isolation systems: piezoelectric stack actuators and voice coil actuators. Piezoelectric actuators are deemed to be of interest due to their inherently high stiffness, which may be beneficial in hard mount systems. On the other hand, voice coil actuators are very versatile and widely available in various designs.

The actuator requirements for the actuator that are discussed are bandwidth, force capacity and noise level. The discussion is focussed on the noise performance (including power amplifiers).

\subsubsection{Actuator requirements}

\section{Bandwidth}

The bandwidth requirement is of course completely analogous to the requirement for the sensors: $0.1-1000 \mathrm{~Hz}$. Most actuators (and their amplifiers) are capable of generating DC forces. Therefore, the bandwidth can alternatively be specified from DC to (at least) $1 \mathrm{kHz}$. This bandwidth requirement is not very stringent and can be met by a large variety of actuators and amplifiers.

\section{Force capacity}

The required force capacity can be estimated from the expected motion levels and the control strategy, which is a combination of feedforward and feedback control.

Recall from section 2.6.2 that the ideal feedforward compensation is given by equation (6.27), where $k_{1}$ is the support stiffness and $X_{0}$ is the floor displacement. However, at frequencies below approximately $1 \mathrm{~Hz}$, no vibration isolation is required. Therefore, equation (6.27) only has to hold for frequencies above $1 \mathrm{~Hz}$.

$$
F_{a}(s)=-k_{1} X_{0}(s)
$$

The support stiffness $k_{1}$ depends on the total machine mass $m$ and the suspension frequency $\omega_{\text {susp }}$. Therefore, the overall peak force amplitude required for feedforward compensation of floor vibrations can be estimated from equation (6.28).

$$
F_{a, \mathrm{pk}} \approx k_{1} x_{0, \mathrm{pk}}=m \omega_{\mathrm{susp}}^{2} x_{0, \mathrm{pk}}
$$


In section 6.1.2, the expected floor acceleration level has been presented (figure 6.2). The RMS acceleration level (in one-third octave bandwidth) between 0.1 and $100 \mathrm{~Hz}$ is expressed by equation (6.29).

$$
\ddot{x}_{0, \mathrm{RMS}}(n)=2 \pi \cdot f(n) \cdot 3 \cdot 10^{-6}, \quad f(n)=(\sqrt[3]{2})^{n}, \quad n=-10 \ldots 20
$$

After conversion to displacement, the RMS floor displacement follows from the square root of the sum of squares, see equation (6.30). The upper and lower bounds $u$ and $l$ on $n$ are chosen according to the frequency range of interest. The expected RMS floor displacement in the frequency range 1-100 $\mathrm{Hz}(l=0, u=20)$ is approximately $0.6 \mu \mathrm{m}$. Above $100 \mathrm{~Hz}$, the contribution to the floor displacement level is negligible.

$$
x_{0, \mathrm{RMS}}=\sqrt{\sum_{n=l}^{u}\left(\frac{\ddot{x}_{0, \mathrm{RMS}}(n)}{(2 \pi f(n))^{2}}\right)^{2}}
$$

Peak forces (and hence peak displacements) are of more interest for determining the required force capacity. Assuming that the floor motion is normally distributed $^{10}$ the $99.9 \%$ confidence interval is $[-3.3 \sigma, 3.3 \sigma]$, where $\sigma$ is the RMS displacement. Moreover, the expected motion level does not describe the resonant behaviour of floors very well, see also figure 6.2. Therefore, an additional ten-fold increase in peak displacement level may be expected. As a result, the peak floor displacement $(1-100 \mathrm{~Hz})$ is likely to be in the order of $20 \mu \mathrm{m}$.

A similar analysis can be performed for the feedback control. It is assumed that the feedback controller is implemented as a direct velocity feedback controller, see equation (6.31). The machine velocity is expressed by $s X_{1}(s)$. The feedback gain $k_{v}$ can be interpreted as a (skyhook) damper, and can be expressed in terms of the machine mass $m$, the suspension resonance frequency $\omega_{\text {susp }}$ and the effective skyhook damping ratio $\zeta_{\text {sky, }}$, see equation (6.32).

$$
\begin{aligned}
& F_{a}(s)=-k_{v} s X_{1}(s) \\
& k_{v}=2 m \zeta_{\text {sky }} \omega_{\text {susp }}
\end{aligned}
$$

Using the expected hard mount machine motion levels of figure 6.2 (dashed line), and assuming a feedback control bandwidth of 1-500 Hz, the RMS machine velocity amounts to approximately $10 \mu \mathrm{m} / \mathrm{s}$. Similarly to the floor displacement, it is expected that peak velocity levels are approximately 33 times higher, i.e. approximately $330 \mu \mathrm{m} / \mathrm{s}$.

\footnotetext{
10 This is justified by the fact that many noise sources contribute to the floor motion. The Central Limit Theorem states that the sum of independent random variables is asymptotically normally distributed.
} 
As an example, the force levels for the laboratory setup are determined, see also section 2.4.2. For a $5 \mathrm{~kg}$ machine with a suspension resonance frequency of $10 \mathrm{~Hz}$, the estimated feedforward peak force level is approximately $0.4 \mathrm{~N}$. Assuming a skyhook damping ratio $\zeta_{\text {sky }}$ of 0.7 , the estimated feedback peak force level is approximately $0.14 \mathrm{~N}$.

It should be noted that the latter force level is an estimate for feedback control only. When the feedforward compensation is in operation, the machine motion levels will decrease and so will the required feedback control force. Therefore, the dynamic force requirements are mostly determined by the feedforward compensation.

The required force levels are very small. However, the levelling forces (required for gravity compensation) are not taken into account in this analysis. Clearly, these forces are many times larger than the dynamic forces required for vibration isolation. Therefore, the static force requirements will most likely dominate the dynamic force requirements for the actuators.

\section{Noise level}

Noise in the actuator's power amplifier and the noise of the digital-analog (DA) converter are converted into random forces, resulting in additional machine motion. Obviously, the equivalent acceleration level should be less than the performance target, which was specified as the residual machine acceleration level when the machine is suspended on a $1 \mathrm{~Hz}$ soft mount.

For a rigid machine, the transfer function from actuator force to machine acceleration is given by equation (6.33). In this section, this transfer function is used to estimate the equivalent acceleration levels due to the various noise sources. As high-frequency dynamics are ignored, it is expected that the estimate provides a lower level for the equivalent acceleration level. It is assumed that the machine is feedback-controlled using skyhook damping. Therefore, the transfer function represents the closed-loop feedback response, i.e. $\zeta_{\text {sky }}$ is the skyhook damping ratio and $\omega_{\text {susp }}$ is the (closed loop) suspension resonance frequency.

$$
\frac{s^{2} X_{1}(s)}{F_{a}(s)}=\frac{1}{m} \frac{s^{2}}{s^{2}+2 \zeta_{\text {sky }} \omega_{\text {susp }} s+\omega_{\text {susp }}^{2}}
$$




\subsubsection{Piezoelectric stack actuator}

In this section, a basic linear model is presented and some typical properties of piezoelectric (PE) stack actuators are discussed. Then, the effects of hysteresis in PE actuators are illustrated. Lastly, the associated electronics and the equivalent noise level are discussed.

\section{Basic model}

The PE stack actuator is modelled as a linear, coupled electrical-mechanical system. Figure 6.13 shows the electrical (left) and mechanical models (right).
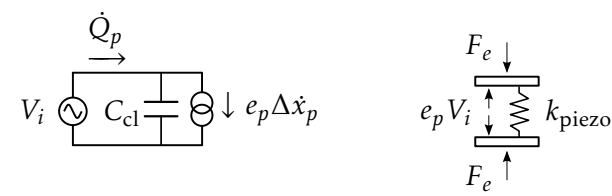

Figure 6.13: Electrical (left) and mechanical (right) model for a PE actuator, valid at frequencies below the first resonance frequency

The electrical model constitutes of a voltage source $V_{i}$, the capacitance $C_{\mathrm{cl}}$ of the PE stack in the clamped state (no elongation possible). The total charge stored in the PE stack is denoted by $Q_{p}$. A current source $e_{p} \Delta \dot{x}_{p}$ is included to model the charge generation due to an elongation $\Delta x_{p}$ of the PE stack.

The (macroscopic) piezoelectric constant $e_{p}$ expresses both the generated charge per unit of deformation as well as the generated force per unit of applied voltage. Therefore, its units are either $[\mathrm{C} / \mathrm{m}]$ or $[\mathrm{N} / \mathrm{V}]$. It generally depends on the material properties as well as the geometry of the PE stack.

Mechanically, the PE actuator is modelled as a force actuator $\left(F_{p}=e_{p} V_{i}\right)$ parallel to a spring $k_{\text {piezo }} . F_{e}$ is the externally applied force, which is also the net force generated by the actuator. A pushing force is taken as a positive force. When necessary, the mass $m_{p}$ of the PE stack can be incorporated in the model by adding two lumped masses at the nodes of the mechanical model. The mechanical model is valid for frequencies below the first resonance frequency of the PE stack. When the frequency range of interest is larger than this first resonance frequency, a more detailed model is required, see e.g. [Adriaens et al. (2000)].

The constitutive equations for this model are given by equation (6.34). The capacitance in the clamped state $C_{\mathrm{cl}}$ is related to the capacitance in the free state $\left(F_{e}=0\right) C_{\mathrm{fr}}$ by equation (6.35). 


$$
\begin{aligned}
& {\left[\begin{array}{l}
F_{e} \\
Q_{p}
\end{array}\right]=\left[\begin{array}{cc}
-k_{\text {piezo }} & e_{p} \\
e_{p} & C_{\mathrm{cl}}
\end{array}\right]\left[\begin{array}{c}
\Delta x_{p} \\
V_{i}
\end{array}\right]} \\
& C_{\mathrm{cl}}=C_{\mathrm{fr}}-\frac{e_{p}^{2}}{k_{\text {piezo }}}
\end{aligned}
$$

The piezoelectric effect is anisotropic in the materials commonly used in PE stack actuators. Therefore, the piezoelectric constant $e_{p}$ depends on the directions of the mechanical load and the electrical field with respect to the polarization of the stack. For stack actuators, the mechanical load and the electrical field are usually parallel to the polarization direction.

\section{Typical properties}

A great advantage of PE actuators is the fact that there is hardly any power required to maintain an arbitrary elongation. Due to the capacitive nature of the PE stack, only a very small leak current is required for static operation. Even though the maximum input voltage may be as high as $150 \mathrm{~V}$ for "lowvoltage" stacks and $1000 \mathrm{~V}$ for high-voltage stacks, this results in a very low power consumption.

The maximum (pk-pk) stroke of PE stacks is typically limited to $1 \%$ of the stack height. Hence, in order to accommodate the expected peak floor displacement of $20 \mu \mathrm{m}$, PE stacks with a height of at least $40 \mathrm{~mm}$ are required. As such stacks are commonly available, this requirement can easily be fulfilled.

Piezoelectric stacks have an inherently high stiffness, typically $10^{7}-10^{10} \mathrm{~N} / \mathrm{m}$. For most machines, directly mounting the machine on PE stacks would therefore result in suspension resonance frequencies far greater than $10 \mathrm{~Hz}$. In order to reduce the mounting stiffness, the actuator may be combined with a transmission mechanism. This is schematically indicated in figure 6.14.

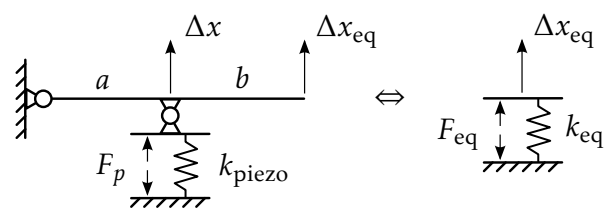

Figure 6.14: Schematic model of piezoelectric actuator with transmission mechanism 


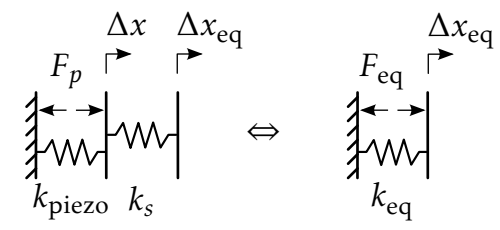

Figure 6.15: Schematic model of piezoelectric actuator with a serial elastic element

The resulting equivalent stiffness, force and displacement are expressed in equation (6.36). For example, amplified piezoelectric actuators (APA) have integrated transmission mechanisms, allowing transmission ratios of 2-20 [Claeyssen et al. (2007)].

$$
\left.\begin{array}{rl}
k_{\mathrm{eq}} & =\frac{k_{\text {piezo }}}{i^{2}} \\
F_{\mathrm{eq}} & =\frac{F_{p}}{i} \\
\Delta x_{\mathrm{eq}} & =i \Delta x
\end{array}\right\} \quad \text { where } i=\frac{a+b}{a}
$$

Another option is to place the actuator in series with a compliant element with stiffness $k_{s}$, see figure 6.15. In this case, the (quasi-stationary) equivalent stiffness and force are given by equation (6.37).

$$
\begin{aligned}
& k_{\text {eq }}=\frac{k_{s}}{k_{s}+k_{\text {piezo }}} k_{\text {piezo }} \\
& F_{\text {eq }}=\frac{k_{s}}{k_{s}+k_{\text {piezo }}} F_{p}
\end{aligned}
$$

Both types of mechanism not only reduce the stiffness, but also result in a decreased force capacity and increased overall stroke. In most cases, the latter property is actually the purpose of the mechanism. It should be noted that the additional mechanical components may be a limiting factor in the useful bandwidth of the "amplified" actuator, due to structural resonances.

\section{Hysteresis}

In piezoelectric actuators that are operated in voltage-mode hysteresis is observed between the voltage input and the stack elongation. This is illustrated in figure 6.16 for a PiezoMechanik PSt 150/5/40 actuator. To measure the hysteresis loop, a $1 \mathrm{~Hz}$ sinusoidal voltage input with varying amplitude has been applied (on top of a $70 \mathrm{~V}$ bias voltage). 


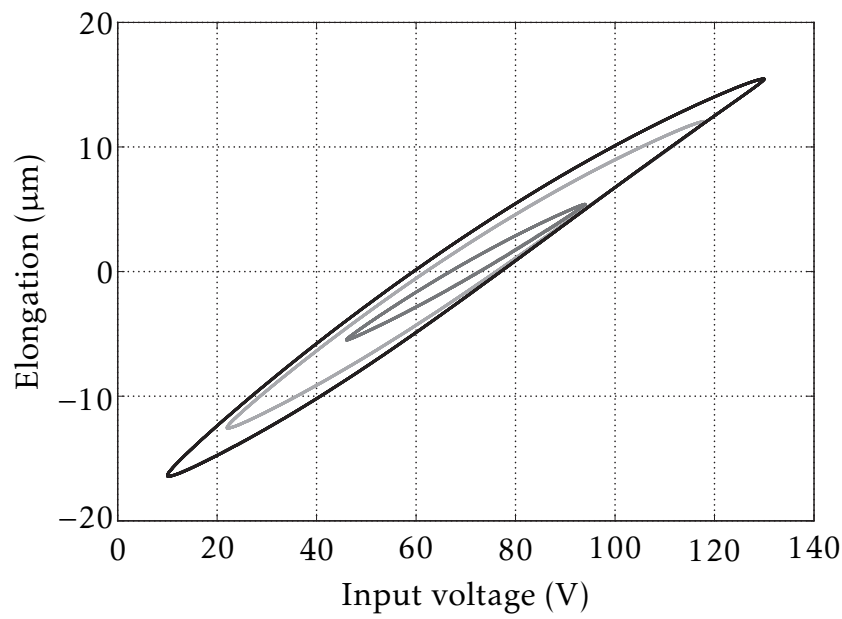

Figure 6.16: Measured hysteresis loops for a single PE actuator, $70 \mathrm{~V}$ bias + $1 \mathrm{~Hz}$ sine wave: $60 \mathrm{~V}$ amplitude (black), $48 \mathrm{~V}$ amplitude (light grey), $24 \mathrm{~V}$ amplitude (dark grey)

The area enclosed by the hysteresis loops is related to the amount of energy that is dissipated during each cycle. This is commonly called hysteretic damping. In high frequency, "large-signal" applications, this power dissipation may lead to significant heating of the stack, which in turn leads to variations in the material properties and eventually to depolarization of the PE stack, resulting in the loss of actuator capability. Clearly, for smaller input amplitudes, the area of the hysteresis loop is greatly reduced. Therefore, heating is of less importance for small-signal applications like vibration isolation.

It is also observed that for smaller input amplitudes the average slope of the loop decreases. This is illustrated more clearly in figure 6.17, where the average slope of the hysteresis loop is shown as a function of the sine wave amplitude for three tested PE actuators (of the same type). The "large-signal" piezoelectric constant of the piezoelectric actuator $(40 \mu \mathrm{m}$ for $150 \mathrm{~V}$ input swing according to the manufacturer's data sheet) is shown for reference.

The small-signal PE constant of the piezoelectric actuators is at least two times less than the large-signal PE constant. This is a significant adverse effect for vibration isolation control, which is inherently a small-signal application. For non-sinusoidal inputs with many more input direction reversals, the effect is likely to be even more significant. Therefore, a reduced actuator effectiveness can be expected when the PE actuator is operated in voltage-mode.

The previously introduced model does not include the hysteresis effect that is observed between the voltage input $V_{i}$ and the stack's elongation $\Delta x_{p}$. In this thesis, the hysteretic effect is accounted for by using a reduced value for the 


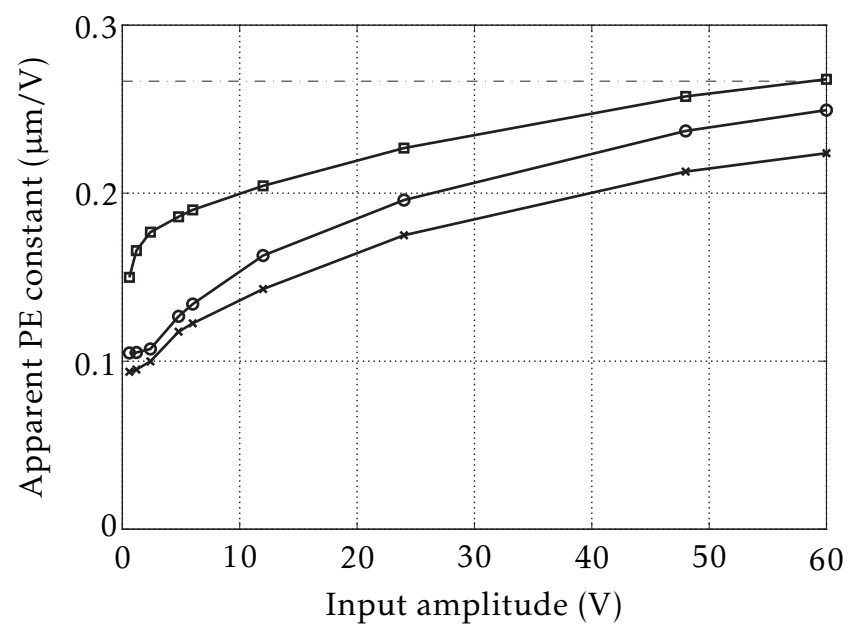

Figure 6.17: Apparent PE constant as a function of input amplitude for three different PE actuators (of the same type) at $1 \mathrm{~Hz}$ sine wave excitation. The large-signal charge constant is shown for reference (dash-dotted)

PE constant $e_{p}$. For background information on the modelling and compensation of the hysteresis effect in piezoelectric actuators the reader is referred to e.g. [Ge and Jouaneh (1995); Goldfarb and Celanovic (1996, 1997); Sain et al. (1997); Adriaens et al. (2000); Banning et al. (2001); Ben Mrad and Hu (2002)].

\section{Electronics}

There are two ways in which a PE actuator can be controlled. The classical method is to use voltage control, more advanced methods use position or charge feedback control.

The voltage control is basically open-loop operation of the PE actuator. The hysteresis effect, as described previously, is fully present. Moreover, the combination of the output impedance of the power amplifier and the capacitance of the PE stack form a first-order low-pass filter, which limits the bandwidth of the actuator.

In closed-loop operation, the linearity of the actuator is greatly improved, i.e. the hysteresis is reduced. The bandwidth of the closed-loop system is now mostly determined by the sensor and controller, see e.g. [Schitter et al. (2002)]. Position feedback control can be based on strain gauges, capacitive sensors or optical sensors. For charge control, the accumulated charge is measured by using a reference capacitor, see e.g. [Fleming and Moheimani (2004); Vautier and Moheimani (2005)]. 
For either open-loop and closed-loop operation, the peak current capability of the amplifier may limit the bandwidth for large, high frequency input signals. For vibration isolation purposes, the noise level of the DA-converter and the power amplifier may be limiting factors.

\section{Noise level}

As an example of this noise performance limit, consider the hardware that is used in the experimental setup: a dSPACE DS2102 DA-converter $( \pm 10 \mathrm{~V}$, 16 bit), a PiezoMechanik SVR150 amplifier (voltage-controlled, $A_{v}=6 \mathrm{~V} / \mathrm{V}$ ) and a PiezoMechanik PSt 150/5/40 PE actuator $\left(e_{p}=3.2 \mathrm{~N} / \mathrm{V}, C_{\mathrm{fr}}=1600 \mathrm{nF}\right.$, $\left.k_{\text {piezo }}=12 \mathrm{~N} / \mu \mathrm{m}\right)$. In the experimental setup, the PE actuator is combined with a serial compliant element with stiffness $k_{s}$ to reduce the suspension resonance frequency, see also equation (6.37). In this section, it is assumed that the suspension resonance frequency lies at $10 \mathrm{~Hz}$, i.e. $k_{s} \approx 4 \cdot 10^{5} \mathrm{~N} / \mathrm{m}$.

Figure 6.18 shows a block diagram of the actuator channel. The input voltage $V_{\text {sig }}$ and the DAC noise $n_{\mathrm{DAC}}$ are first amplified. The output impedance $R_{o}$ of the amplifier $(1 \mathrm{k} \Omega)$ and the capacitance $C_{\mathrm{fr}}$ of the PE actuator form a first order low-pass (LP) filter for the amplified signal and the amplifier noise $n_{\text {AMP }}$. The voltage over the PE stack is then converted to an equivalent force. Lastly, a high-pass (HP) filter is added to model the acceleration response of the machine, see also equation (6.33).

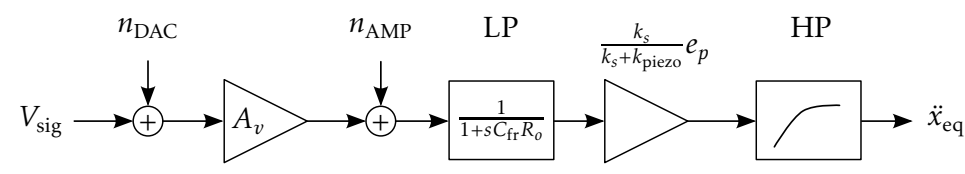

Figure 6.18: Block diagram of the PE actuator channel

The noise levels of the SVR150 amplifier and the DS2102 DA-converter are obtained experimentally. The resulting equivalent 1/3-octave RMS acceleration levels for both noise sources are shown in figure 6.19, together with the reference soft mount machine acceleration level.

The DAC noise has the largest contribution to the total actuator channel noise. It consists of white noise and significant contributions at the power supply frequency and its (first) higher harmonics. The amplifier noise is also dominated by higher harmonics of the power supply frequency.

However, both noise levels are significantly smaller than the vibration isolation target. It can therefore be concluded that the noise level of this particular $\mathrm{PE}$ actuator channel is adequate for vibration isolation purposes. It should be noted that this remarkably low noise level is caused mostly by the scale factor $\frac{k_{s}}{k_{s}+k_{\text {piezo }}}$, which has a value of approximately $3.3 \cdot 10^{-3}$ for this example. 


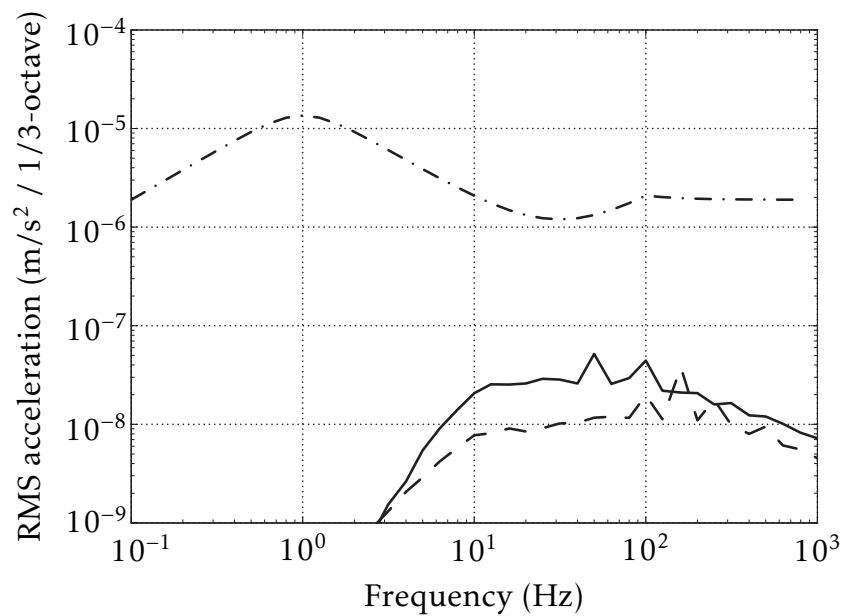

Figure 6.19: Equivalent acceleration due to various noise sources in the PE actuator channel

— : DS2101 DAC noise

- - : SVR150 amplifier noise

$-\cdot-$ : reference soft mount machine acceleration level

\subsubsection{Voice coil actuator}

Another common type of actuator is the voice coil actuator (VCA), which basically consists of a permanent magnet and a coil. The force generated by the current $\dot{Q}_{c}$ in the coil is expressed by equation (6.38), where $k_{m}$ is the VCA motor constant.

$$
\begin{aligned}
& F=k_{m} \dot{Q}_{c} \\
& V_{\text {ind }}=-k_{m} \Delta \dot{x}_{c}
\end{aligned}
$$

Mechanically, the voice coil actuator can be modelled as a force actuator. The VCA electrical model is shown in figure 6.20. The coil is modelled as the series connection of a resistor $R_{c}$ and an inductance $L_{c}$. Moreover, an additional voltage source $V_{\text {ind }}$ is included to model the induced back-emf voltage due to the velocity difference $\Delta \dot{x}_{c}$ between the coil and the magnet, see also equation (6.39). The input voltage is denoted by $V_{i}$. 


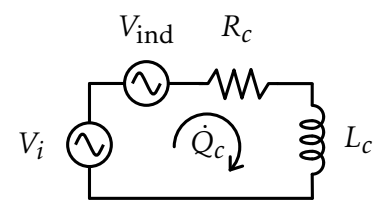

Figure 6.20: Electrical model of a voice coil actuator

\section{Typical properties}

Compared to a PE actuator, a voice coil actuator can generate less force (for a comparable volume) but has a much larger stroke (typically several millimetres). Moreover, voice coil actuators are practically free of hysteresis. The motor constant $k_{m}$ depends on the magnetic field strength of the permanent magnet, the coil geometry and on the position of the coil with respect to the magnet [Riedesel et al. (1990), App. 1]. However, due to the small vibration amplitudes in typical vibration isolation systems, it is allowed to consider $k_{m}$ as a constant. Therefore, a voice coil actuator can be modelled as a linear actuator.

In contrast to PE actuators, a voice coil actuator does consume power to hold a non-equilibrium position. During dynamic operation, power is also dissipated due to the coil resistance $R_{c}$. As a result, the thermal limits of a voice coil actuator usually determine its suitability for a particular application.

\section{Electronics}

Just like PE actuators, voice coil actuators can be operated in open-loop or in closed-loop configuration. In an open-loop configuration, the voice coil actuator is said to be voltage controlled. Assuming an ideal voltage source (zero impedance), an induced voltage generates an electromotive force $F_{\text {emf }}$, as indicated by equation (6.40).

$$
F_{\text {emf }}=\frac{k_{m}}{R_{c}+L_{c} s} V_{\text {ind }}=-\frac{k_{m}^{2}}{R_{c}+L_{c} s} \Delta \dot{x}_{c}
$$

This force can be interpreted as a frequency-dependent damping force. The equivalent mechanical model of a voice coil actuator in open-loop configuration is shown in figure 6.21.

In case of a current-controlled configuration, the current through the coil is measured and used in a feedback loop. Due to the high feedback gain, the electromotive force $F_{\text {emf }}$ is almost completely eliminated (within the bandwidth of the feedback loop). Hence, in closed-loop configuration, the voice coil actuator effectively remains a force actuator. 


$$
\frac{k_{m}}{R_{c}+L_{c} s} V_{i} \sum_{\downarrow}^{\uparrow} k_{m}^{2} / L_{c}
$$

Figure 6.21: Equivalent mechanical model for a voice coil actuator in openloop (voltage-controlled) configuration

\section{Noise level}

Similar to the PE actuator, the equivalent acceleration noise level of the VCA channel is determined by the DAC noise and the amplifier noise. Figure 6.22 shows the block diagram models for a voltage-controlled and currentcontrolled VCA channel.

In figure 6.22a, the actuator channel using a voltage-controlled VCA is shown. The input signal $V_{\text {sig }}$ and the DAC noise are fed through the amplifier, which can be modelled as a voltage gain $A_{v}$ in the bandwidth of interest. Filtering the amplified voltage signal and the amplifier output noise $n_{\text {AMP }}^{v}$ by the coil admittance $^{11}$, results in the current through the coil. Then, multiplication by the motor constant $k_{m}$ gives the generated force and finally, the equivalent acceleration is found by filtering through the high-pass system response (HP), see also equation $(6.33)^{12}$.

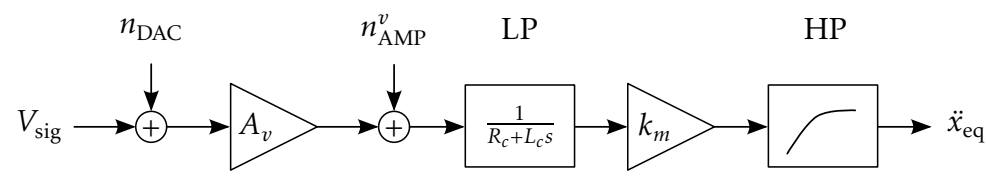

(a) Voltage-controlled VCA

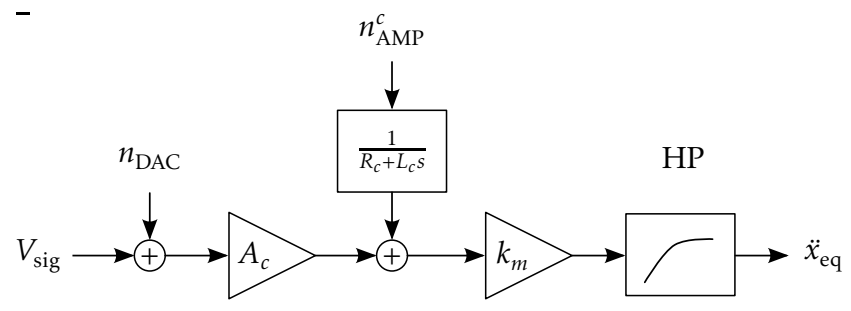

(b) Current-controlled VCA

Figure 6.22: Block diagrams of the VCA channel

11 Admittance is the inverse of impedance

12 It can be shown that the frequency-dependent damping of equation (6.40) does not significantly alter the system response 
In figure $6.22 \mathrm{~b}$, a similar block diagram is shown for the current-controlled case. In this case, the relation between an input voltage and the current through the coil can be modelled as a pure gain $A_{c}$ within the bandwidth of the amplifier. The response for the amplifier output noise remains the same, with the exception that the output noise $n_{\mathrm{AMP}}^{c}$ is now determined while the current feedback loop is active.

As an example, the noise level is once more determined for the hardware which has been tested in the laboratory setup. The voice coil actuator is a GeePlus $(G+)$ VM4032-250 model $\left(k_{m}=11.2 \mathrm{~V} /(\mathrm{m} / \mathrm{s}), R_{c}=12.8 \Omega, L_{c} \approx 3\right.$ $\mathrm{mH})$. The accompanying Trust Automation TA-105 power amplifier can be operated in either current mode $\left(A_{c}=0.05 \mathrm{~A} / \mathrm{V}\right.$, bandwidth $\left.\sim 5 \mathrm{kHz}\right)$ or voltage mode $\left(A_{v}=13.6 \mathrm{~V} / \mathrm{V}\right)^{13}$.

Figure 6.23 shows the equivalent acceleration levels due to amplifier noise for the voltage- and current-controlled configurations. Clearly, the voltagecontrolled configuration generates less noise, and is therefore preferred over the current-controlled configuration.

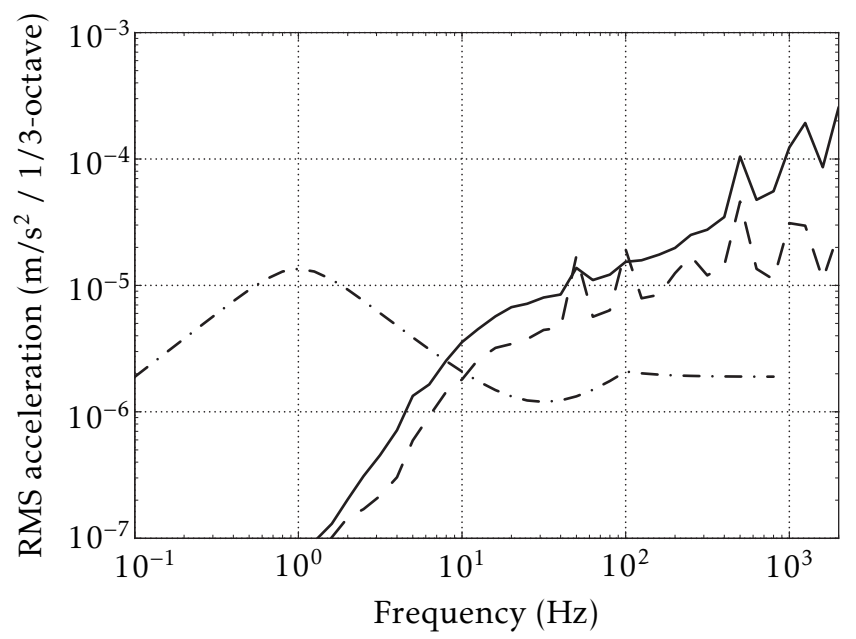

Figure 6.23: Equivalent acceleration due to amplifier noise in the VCA channel

- - : voltage-controlled voice coil actuator

— : current-controlled voice coil actuator

$-\cdot-$ : vibration isolation target

\footnotetext{
13 To reduce the overall gain in voltage mode, an additional voltage gain of 0.05 has been included between the DAC and the power amplifier (by means of a voltage divider). Hence, the effective value for $A_{v}$ is $0.68 \mathrm{~V} / \mathrm{V}$.
} 
However, the equivalent acceleration levels of both configurations clearly exceed the vibration isolation target at frequencies above $10 \mathrm{~Hz}$. This is partially caused by the fact that the voice coil actuator and its amplifier are not matched well to the machine mass of the experimental setup. The continuous force capacity of this particular actuator is $10 \mathrm{~N}$, which is an order of magnitude larger than the required peak force, see section 6.2.1. Hence, the same actuator configuration could be used for a much larger machine mass. This would result in a reduced equivalent acceleration level, as the equivalent acceleration level is inversely proportional to machine mass. Even then, it may be expected that the amplifier noise poses a performance limit for frequencies above approximately $100 \mathrm{~Hz}$.

The same DA-converter is used as in the setup with the PE actuator. The resulting equivalent acceleration level is less than $10 \mathrm{~nm} / \mathrm{s}^{2}$ per one-third octave at frequencies below $1 \mathrm{kHz}$, for both the voltage- and current-controlled case. This noise level is clearly negligible compared to the amplifier noise.

\subsubsection{Recapitulation and final remarks}

In this section, requirements regarding bandwidth, force capacity and noise level (of the associated electronics) have been discussed for two types of actuators: piezoelectric stack actuators and voice coil actuators. It has been argued that the bandwidth and force capacity requirements can be easily fulfilled. The noise level requirement, which is related to the vibration isolation target, is not so easily satisfied.

It has been shown that the piezoelectric actuator configuration has a very low noise level, due to the stiffness reduction mechanism used in the experimental setup. Unfortunately, this stiffness reduction mechanism and the hysteresis effect in the piezoelectric actuator significantly reduce the force capacity of the actuator.

The noise equivalent acceleration level of the voice coil actuator is much larger than the machine acceleration level of the reference soft mount system. This is partially due to the poor matching of the actuator and amplifier to the machine mass. It is expected that even with an improved matching the noise level poses a limitation on the achievable performance at frequencies above approximately $100 \mathrm{~Hz}$.

It is interesting to note that, in terms of noise performance, operation of the voice coil actuator in open-loop (voltage mode) is preferred over closed-loop operation (current mode). 



\section{Chapter 7}

\section{Experimental setup}

In this chapter, the experimental setup is described that is used to test the real-time implementation of the various control algorithms that have been presented in chapters 4 and 5. In section 7.1, a general description of the setup is presented. Then, the parametric modelling of the setup is discussed in section 7.2. The parametric model is mainly used to gain insight in the system dynamics. However, for feedback control design and the implementation of the feedforward controller more accurate models are required. These models are obtained from system identification experiments, which are discussed in section 7.3.

\subsection{Description of the setup}

In section 2.4, the basic system model has been presented, which is the most basic representation of a machine with a dominant structural resonance frequency at approximately $100 \mathrm{~Hz}$, which is mounted on a floor by means of a hard mount, i.e. the suspension resonance frequency $f_{\text {susp }}$ is relatively large. The experimental setup is designed to represent this basic system model. In order to limit the complexity of the dynamic behaviour, the setup is restricted to allow motion in one direction only.

As mentioned in chapter 6, two types of actuators are used in the experimental setup: a piezoelectric actuator (PEA) and a voice coil actuator (VCA). For this purpose, the components that make up the hard mount can be exchanged. 


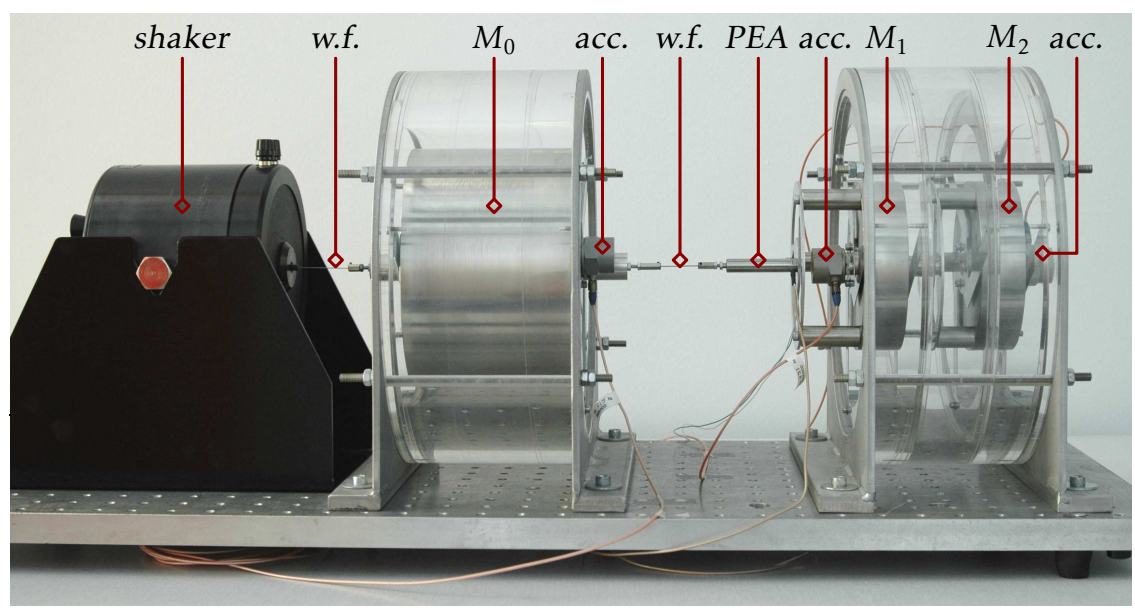

Figure 7.1: Experimental setup with piezoelectric actuator (PEA), $M_{0}$ : floor body, $M_{1}$ : machine body 1, $M_{2}$ : machine body 2, acc.: Endevco 7703A-1000 accelerometer, w.f.: wire flexure

Figure 7.1 shows an overview picture of the experimental setup with the piezoelectric actuator. Just like the basic system model of section 2.4 , the setup consists mainly of three bodies $M_{0}, M_{1}$ and $M_{2}$, which represent the floor and the two machine bodies respectively. The setup is placed horizontally to circumvent the necessity of gravity compensation.

The machine bodies and the floor body are suspended in acrylic glass housings by leaf springs that provide linear guidance for each body. In figure 7.2, the linear guidance mechanism for each of the bodies is illustrated conceptually. Each set of three leaf springs $(1-3,4-6)$ has been made in one piece from spring steel sheet metal, using laser cutting.

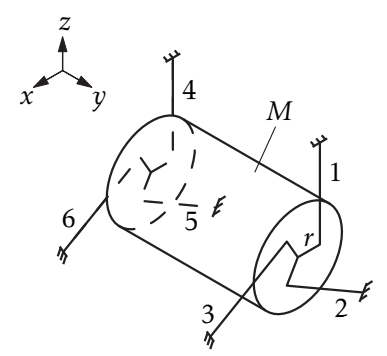

Figure 7.2: Linear guidance concept: a rigid, cylindrical body $M$ is suspended by six leaf springs (1-6), allowing motion along the $y$-axis only (combined with a small rotation around the $y$-axis). Each leaf spring is mounted at a distance $r$ from the body's $y$-axis, at $120^{\circ}$ relative (in-plane) angles. 


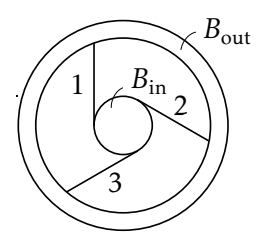

Figure 7.3: Schematic front view of the compliant component in the PEA hard mount. The outer body $B_{\text {out }}$ and the inner body $B_{\text {in }}$ are connected by three flexures (1-3).

The connection between the floor body $M_{0}$ and the machine body $M_{1}$ constitutes the hard mount suspension. In this PEA-based setup, it consists of a wire flexure, the piezoelectric actuator (PEA) and a serial compliant component. The wire flexure is used to compensate for any alignment errors between $M_{0}$ and $M_{1}$. The serial compliant component is used to reduce the overall suspension stiffness to the desired value, as discussed in section 6.2.2. The compliant component is not clearly visible in figure 7.1 , because it is seen from the side. In figure 7.3, a schematic front view of this compliant component is presented. The three flexures allow out-of-plane motion of the inner body $B_{\text {in }}$ with respect to the outer body $B_{\text {out }}$. The entire component is manufactured in one piece by laser cutting.

The machine bodies $M_{1}$ and $M_{2}$ are connected by a similar compliant component as used in the hard mount. Due to this compliant connection, a clear structural resonance mode is realized in the setup.

The acceleration of each body is measured by an Endevco 7703A-1000 accelerometer. The sensor on the floor body $M_{0}$ serves as the reference signal for the adaptive feedforward controller. The sensor on the machine body $M_{1}$ is used as the feedback sensor, as well as the error sensor for the adaptive feedforward control. Finally, the acceleration signal from the sensor on the machine body $M_{2}$ is used for validation of the vibration isolation performance.

The sensors on $M_{1}$ and $M_{2}$ are mounted on the $y$-axes of these bodies (see figure 7.2 for the coordinate system). Due to space limitations, the sensor on the floor body $M_{0}$ is mounted $35 \mathrm{~mm}$ off-centre. In section 7.2.2, it is demonstrated that the sensitivity of this sensor to rotations of the floor body $M_{0}$ is increased significantly.

An electrodynamic shaker (Brüel \& Kjær Type 4809) serves as the floor vibration source. It is connected to the floor mass $M_{0}$ by a wire flexure, which is used to overcome any alignment errors.

The shaker and the housings of the floor and machine bodies are mounted on an aluminium base plate, which in turn is placed on a laboratory table. 


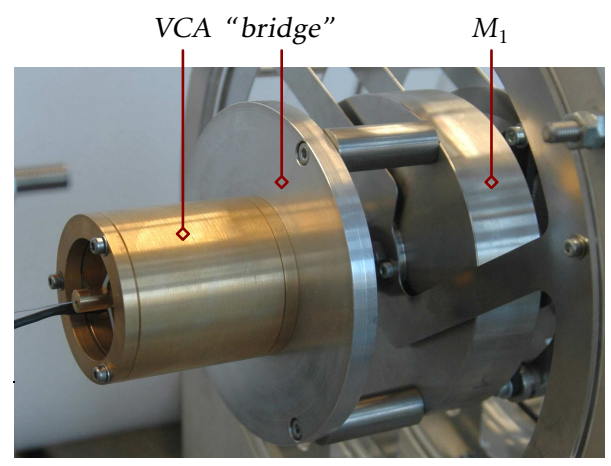

Figure 7.4: Close-up of the voice coil actuator (VCA) assembly, which replaces the PEA assembly in figure 7.1

Figure 7.4 shows a detail of the setup where the piezoelectric actuator is replaced by the voice coil actuator (VCA) assembly. The voice coil is guided with respect to the VCA permanent magnet by a set of flexures, similar to the concept shown in figure 7.2. The serial compliant component in the PEA hard mount is replaced by a "bridge" structure, which is required to allow room for the accelerometer mounted on the first machine body $M_{1}$.

\subsection{Parametric modelling}

In order to gain insight into the setup's dynamic behaviour (with either the piezoelectric or the voice coil actuator), parametric models have been developed. As the setup is designed to have a single direction of motion, a singledirectional mechanical model is discussed first in section 7.2.1. Then, in section 7.2.2, the effects of three-dimensional coupled dynamics on the inputoutput behaviour are discussed.

For the controller design, a discrete time model of the setup is required, which accurately describes the dynamic behaviour of the setup in the frequency range to at least $1 \mathrm{kHz}$. Besides the mechanical system behaviour, this model includes the sensor and actuator electronics as well. The modelling of the electrical components is discussed briefly in section 7.2.3. Finally, the inputoutput behaviour of the combined electromechanical models is presented in section 7.2.4. 


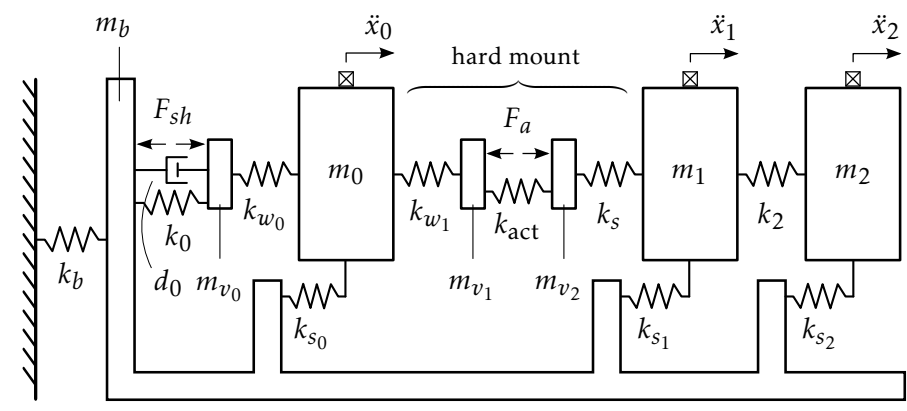

Figure 7.5: Lumped parameter model of the experimental setup, for the principal direction of motion. The accelerometers in the setup are indicated by $\otimes$. The meaning of the model parameters is explained in the text.

\subsubsection{Single-directional model}

In this section, a single-directional model of the experimental setups is presented. It uses lumped mass elements, linear spring elements and viscous damper elements to model the dominant dynamic behaviour. Due to the similarity in the structure of the PEA assembly and the VCA assembly, the same model structure can be used for both setups and only the parameter values need to be adapted. The model structure is shown in figure 7.5.

The floor and machine bodies $M_{0}, M_{1}$ and $M_{2}$ are modelled by the mass elements $m_{0}, m_{1}$ and $m_{2}$. The compliant component connecting $M_{1}$ and $M_{2}$ is modelled by the spring element $k_{2}$. The linear guidance stiffness for each body is modelled by the spring elements $k_{s_{0}}, k_{s_{1}}$ and $k_{s_{2}}$ respectively. These spring elements are connected to the mass element $m_{b}$, which represents the total mass of the housings of the machine bodies, the aluminium base plate and the shaker stator. ${ }^{1}$ The connection of the base plate to the laboratory table (which is assumed to be fixed) is represented by the spring element $k_{b}$. However, in the remainder of this section, it is assumed that the base plate is rigidly connected to the laboratory table.

The shaker is modelled as a force actuator $F_{s h}$ parallel to a stiffness element $k_{0}$, which represents the shaker's internal linear guidance system. Because the shaker is operated in voltage mode, the back-emf in the shaker's coil introduces damping. Moreover, a rubber seal is mounted between the shaker housing and the shaker's moving element, which also adds significant damping. The viscous damper $d_{0}$ models both these damping contributions. The shaker's moving element is modelled by the mass element $m_{v_{0}}$. The wire flexure that is used to overcome alignment errors is modelled by the stiffness element $k_{w_{0}}$.

1 Note that these components are assumed to be rigidly connected. 
The hard mount assembly is modelled by three spring elements, two mass elements and a force actuator $F_{a}$, as indicated in figure 7.5.

In case of the PEA-based setup, $k_{\text {act }}$ represents the stiffness of the piezoceramic stack. The compliant component placed in series with the piezoelectric actuator is modelled by $k_{s}$. The spring $k_{w_{1}}$ represents the wire flexure that is used to overcome any misalignment between the floor body $M_{0}$ and the actuator assembly. The mass elements $m_{v_{1}}$ and $m_{v_{2}}$ represent the equivalent lumped masses of the piezoceramic stack, the actuator casing and some fastening elements.

In case of the VCA-based setup, the spring $k_{\text {act }}$ is used to model the stiffness of the voice coil linear guidance flexures. Once more, the stiffness $k_{w_{1}}$ represents a wire flexure, which is used to overcome any misalignment between the floor body $M_{1}$ and actuator assembly. The bridge structure is assumed to have a limited stiffness, which is modelled by the spring element $k_{s}$. The mass element $m_{v_{1}}$ represents the mass of the coil itself. The lumped mass of the permanent magnet, the magnet housing and the bridge structure is modelled by $m_{v_{2}}$.

Values of the model parameters for both setups can be found in appendix A.2, in table A.1. These parameter values have either been collected from data sheets, computed from CAD data or computed by finite element analysis.

\section{Input-output behaviour in the frequency domain}

The dynamical behaviour of the setup is described in the frequency domain by six transfer functions, as defined in equation (7.1). These transfer functions are easily computed using the method discussed in appendix A.1.2.

The resulting frequency response of $\boldsymbol{H}_{m}(s)$ is shown in figure 7.6 for both the VCA-based setup (solid line) and the PEA-based setup (dashed line).

$$
\boldsymbol{H}_{m}(s)=\left[\begin{array}{ll}
H_{11}(s) & H_{12}(s) \\
H_{21}(s) & H_{22}(s) \\
H_{31}(s) & H_{32}(s)
\end{array}\right] \equiv\left[\begin{array}{ll}
\frac{\ddot{X}_{0}(s)}{F_{s h}(s)} & \frac{\ddot{X}_{0}(s)}{F_{a}(s)} \\
\frac{\ddot{X}_{1}(s)}{F_{s h}(s)} & \frac{\ddot{X}_{1}(s)}{F_{a}(s)} \\
\frac{\ddot{X}_{2}(s)}{F_{s h}(s)} & \frac{\ddot{X}_{2}(s)}{F_{a}(s)}
\end{array}\right]
$$

The dominant resonance frequencies in the VCA-based setup are 4.4, 19 and $88 \mathrm{~Hz}$, which are all clearly visible in e.g. figure 7.6c (solid line). The accompanying resonance modes are respectively the floor mode, suspension mode and (first) structural mode. The additional dynamics above $1 \mathrm{kHz}$ are resonances associated with the wire flexures and the bridge structure. 

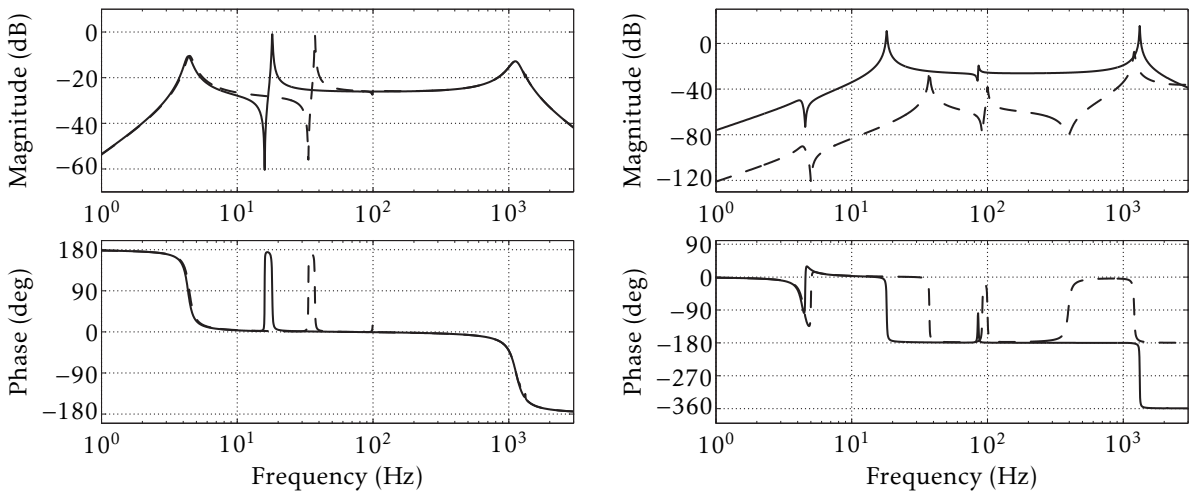

(a) $H_{11}(s)=\ddot{X}_{0}(s) / F_{s h}(s)$

(b) $H_{12}(s)=\ddot{X}_{0}(s) / F_{a}(s)$
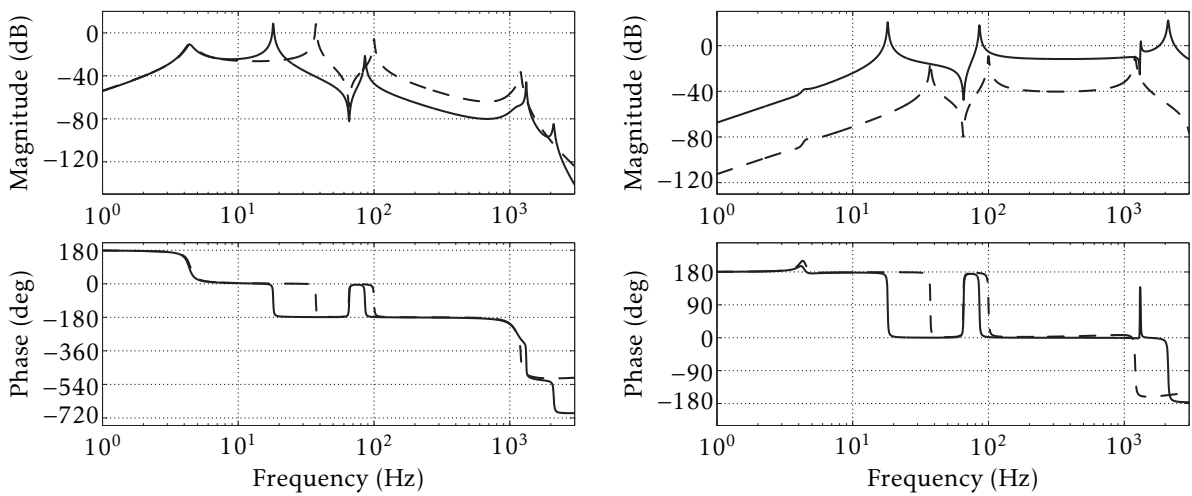

(c) $H_{21}(s)=\ddot{X}_{1}(s) / F_{s h}(s)$

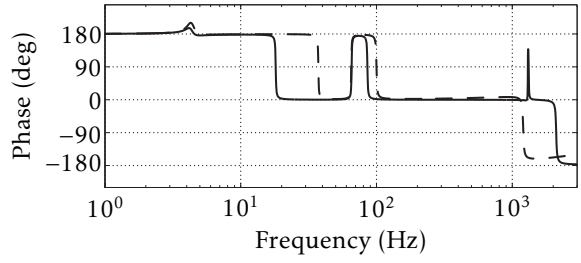

(d) $H_{22}(s)=\ddot{X}_{1}(s) / F_{a}(s)$
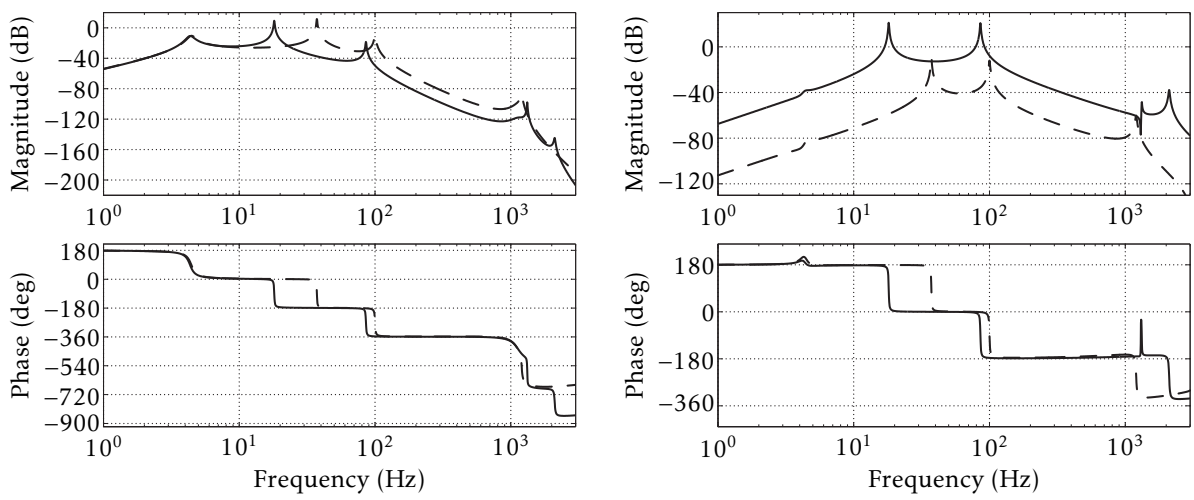

(e) $H_{31}(s)=\ddot{X}_{2}(s) / F_{s h}(s)$

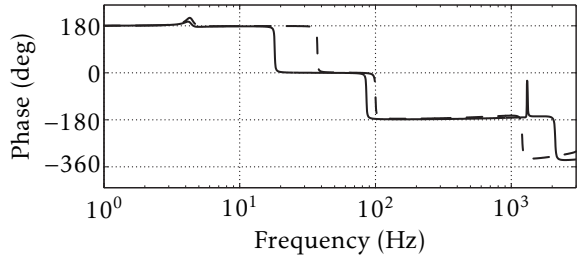

(f) $H_{32}(s)=\ddot{X}_{2}(s) / F_{a}(s)$

Figure 7.6: Bode diagrams of the single-directional mechanical models of the experimental setups (see figure 7.5); _ : VCA-based; - - : PEA-based 
For the PEA-based setup, the dominant resonance frequencies are 4.5, 37 and $99 \mathrm{~Hz}$ respectively, for the floor mode, suspension mode and (first) structural mode. Once more, some additional dynamics due to the wire flexures occur above $1 \mathrm{kHz}$.

An obvious difference compared to the VCA-based setup is the higher suspension mode frequency, which is caused by the higher suspension stiffness in the PEA-based setup. In figures 7.6c and 7.6e, it is seen that the increased suspension stiffness increases the high frequency response to the shaker force (i.e. to floor vibrations). Moreover, it is observed in figures 7.6b, 7.6d and 7.6f that much more force is required from the piezoelectric actuator than from the voice coil actuator to generate a desired acceleration. This is partly caused by the increased suspension stiffness, but the most important reason is the presence of the serial compliant component. ${ }^{2}$ As a result, much larger forces are required in the PEA-based setup (compared to the VCA-based setup) to achieve the same vibration isolation performance.

Both setups have suspension resonance frequencies (19 and $37 \mathrm{~Hz}$ respectively) that are considerably larger than that of a soft mounted system (typically $1 \mathrm{~Hz}$ ). As a result, the support stiffness of these setups are respectively approximately 350 and 1350 times larger than that of a typical soft mount system.

The transfer function $\mathrm{H}_{22}(s)$ (figure 7.6d) describes the mechanical part of the dynamics that are involved in the feedback loop (see e.g. figure 4.3a). Recall from section 4.3.1 that, with collocated actuator/sensor pairs, DVF-like feedback control results in a robustly stable closed loop system. It has also been discussed that the collocation condition is violated when absolute motion sensors are used, but that a practically collocated system may still be achieved.

In this case, the system transfer function should at least exhibit an alternating pole-zero pattern. In figure $7.6 \mathrm{~d}$, an alternating pole-zero pattern is indeed observed in the frequency range from 10 to $1000 \mathrm{~Hz}$, for both the PEA-based and VCA-based setups.

Above $1 \mathrm{kHz}(1200 \mathrm{~Hz}$ for the PEA-based setup and at $2 \mathrm{kHz}$ for the VCAbased setup), an internal resonance occurs that deviates from the collocated behaviour. This resonance is associated with the spring element $k_{s}$ (see figure 7.5), i.e. with the serial component in the PEA-based setup or the bridge structure in the VCA-based setup.

The near pole-zero cancellation at $4.5 \mathrm{~Hz}$ in both transfer functions is of little significance because the accompanying phase change is only limited (due to the significant damping of the poles and zeros that are involved).

Therefore, it can be concluded that a robustly stabilizing feedback controller can be designed, provided that the control bandwidth is limited to approximately $300 \mathrm{~Hz}$.

2 See equation (6.37) for the effect of the serial stiffness $k_{s}$ on the effective force. 


\subsubsection{Coupled 3D dynamics}

So far, the mechanical model has only considered motion in one direction, under the assumption that the major bodies' motion is perfectly linear, i.e. the off-axis motion of the bodies is completely constrained. In practice, some off-axis motion, e.g. tilting and rocking, occurs due to the finite stiffness of the linear guidance in these directions of motion. These off-axis resonance modes are excited by the actuators as a result of alignment errors in the system. Moreover, due to the tilt-to-horizontal coupling in the accelerometers (recall section 6.1.1), the sensors can measure these off-axis motions.

Three-dimensional models of both the PEA-based and VCA-based setup have been constructed, using the software package SPACAR. These models describe the stiffness of the linear guidance of each major body, resulting in many offaxis resonances (e.g. tilt) as described above. The model also incorporates the tilt-to-horizontal coupling effect in the accelerometers. Lastly, alignment and orientation errors of the floor and the machine bodies are included. These errors have been varied over $\pm 1 \mathrm{~mm}$ and $\pm 10 \mathrm{mrad}$, respectively.

It is beyond the scope of this thesis to discuss the actual $3 \mathrm{D}$ modelling in SPACAR. Instead, an example of the resulting effects on the input-output behaviour is illustrated in figure 7.7 .

Compared to figure 7.6, the contributions of the off-axis resonance modes are clearly observed as "spikes" in the frequency range between 200 and $400 \mathrm{~Hz}$. Due to the off-centre placement of the sensor on the floor body $M_{0}$, the most significant contributions occur in $H_{11}(s)$ (figure 7.7a) and $H_{12}(s)$ (figure 7.7b).

It is important to note that the coupled dynamics can sometimes result in nonminimum phase zeros, e.g. as observed in the phase plot of figure $7.7 \mathrm{f}$ (solid line, at $500 \mathrm{~Hz}$ ). When such non-minimum phase behaviour would occur in the transfer function $\mathrm{H}_{22}(\mathrm{~s})$, it would pose a (possibly severe) constraint on the feedback performance (see e.g. [Skogestad and Postlethwaite (2005), Sect. 5.2]. Moreover, recall from chapter 5 that non-minimum phase zeros in either $H_{11}(s)$ and $H_{22}(s)$ can not be stably inverted and would therefore restrict the achievable performance of the (adaptive) feedforward controller.

As a consequence, careful alignment of the actuators and sensors in the hard mount is required to prevent any non-minimum phase zeros from occurring in the relevant transfer functions $H_{11}(s)$ and $H_{22}(s)$. 

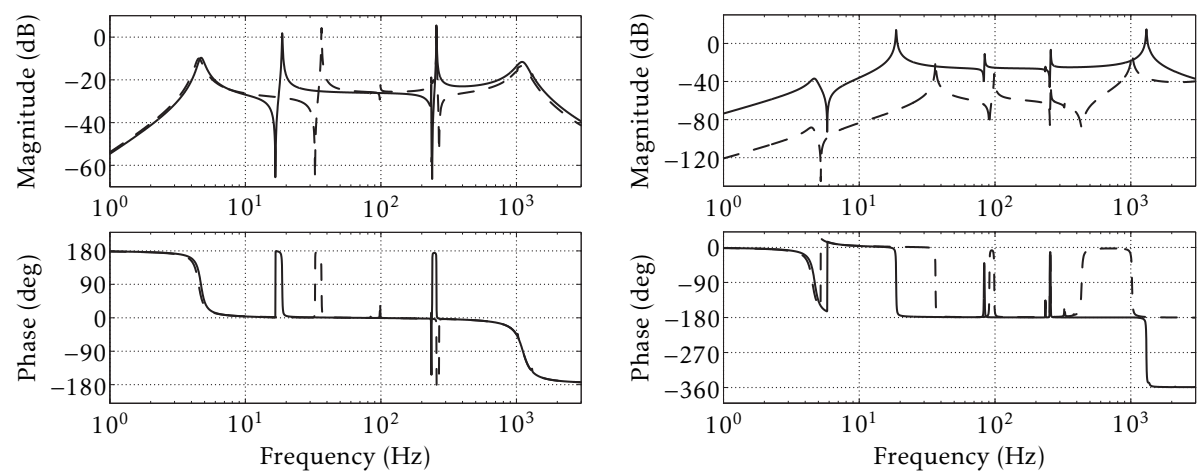

(a) $H_{11}(s)=\ddot{X}_{0}(s) / F_{s h}(s)$

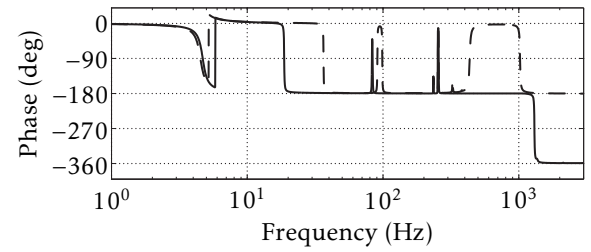

(b) $H_{12}(s)=\ddot{X}_{0}(s) / F_{a}(s)$
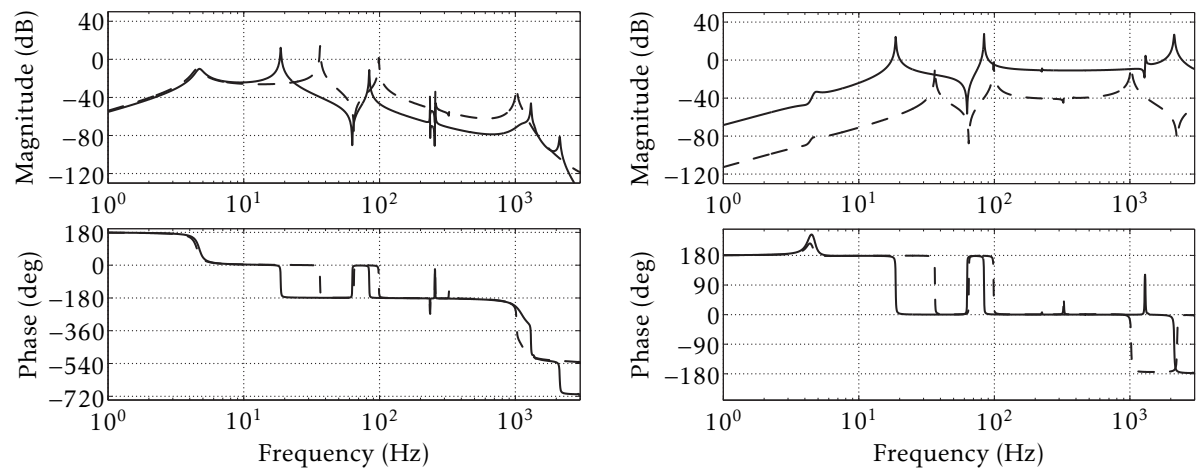

(c) $H_{21}(s)=\ddot{X}_{1}(s) / F_{s h}(s)$

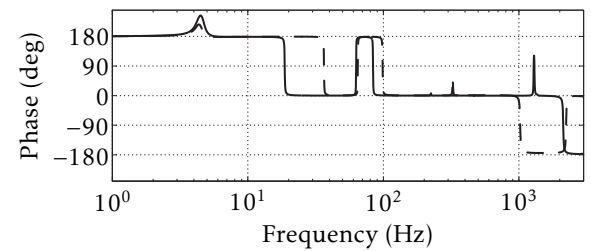

(d) $H_{22}(s)=\ddot{X}_{1}(s) / F_{a}(s)$
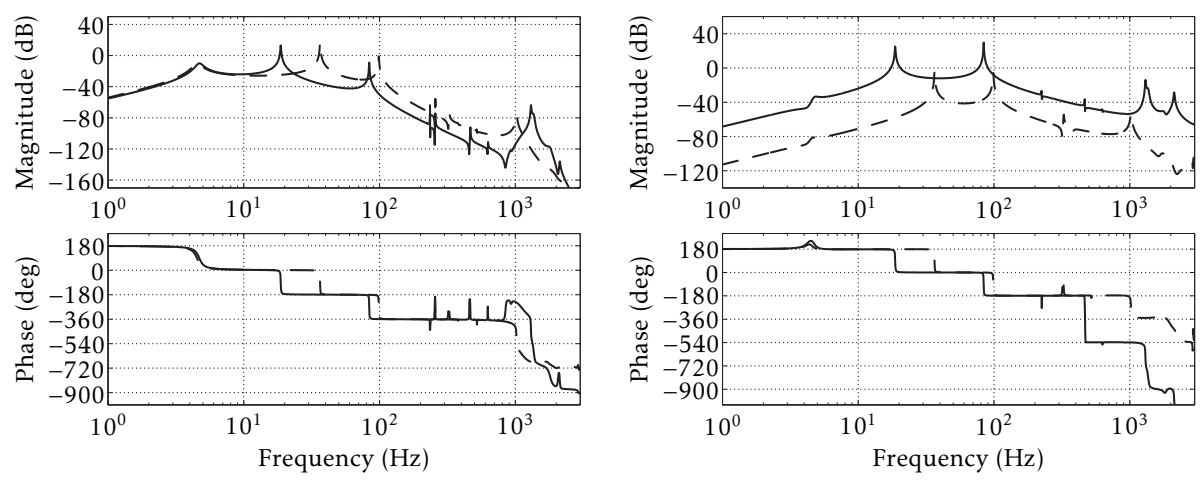

(e) $H_{31}(s)=\ddot{X}_{2}(s) / F_{s h}(s)$

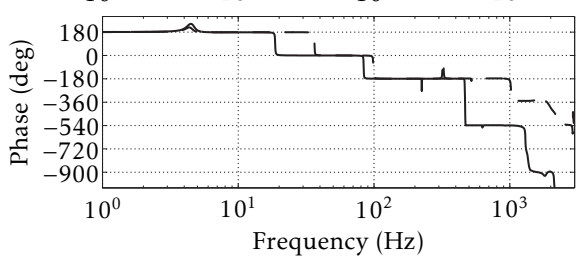

(f) $H_{32}(s)=\ddot{X}_{2}(s) / F_{a}(s)$

Figure 7.7: Bode diagrams of the 3D mechanical models of the experimental setups; — : setup with VCA; - - : setup with PEA 


\subsubsection{Signal conditioning and power electronics}

Finally, the model is extended with the signal conditioning and power electronics. A block diagram showing the interconnection of the mechanical system and the sensor and actuator electronics is shown in figure 7.8. The input signals $u_{s h}$ and $u_{a}$ represent the digital driving signals for the shaker and the (PEA or VCA) actuator. The output signals $y_{0}, y_{1}$ and $y_{2}$ are sampled versions of the sensor signals, which represent the accelerations of the floor mass $M_{0}$, the first machine mass $M_{1}$ and the second machine mass $M_{2}$ respectively. ${ }^{3}$

The output signals are then related to the input signals by six transfer functions $\mathrm{n}$ the $z$-domain, as indicated in equation 7.2. The naming of the primary path $P(z)$, secondary path $S(z)$, tertiary path $T(z)$ and quaternary path $Q(z)$ is carried over from the discussion on adaptive feedforward control in chapter 5. The two remaining transfer functions $P_{2}(z)$ and $S_{2}(z)$ model the effect of the actuators on the acceleration $\ddot{x}_{2}$ of the second machine mass $M_{2}$.

$$
\left[\begin{array}{c}
Y_{0}(z) \\
Y_{1}(z) \\
Y_{2}(z)
\end{array}\right]=\left[\begin{array}{ll}
T(z) & Q(z) \\
P(z) & S(z) \\
P_{2}(z) & S_{2}(z)
\end{array}\right]\left[\begin{array}{c}
U_{s h}(z) \\
U_{a}(z)
\end{array}\right]
$$

The signal $y_{2}$ (i.e. the acceleration $\ddot{x}_{2}$ ) is used as the validation signal for the vibration isolation performance. Recall from section 3.1 that the improvement of the deformation transmissibility is the objective that determines the overall vibration isolation performance. Unfortunately, it is also the most challenging to verify, because there are no displacement sensors incorporated in the setup that can measure the deformation directly. However, the acceleration $\ddot{x}_{2}$ of the second machine mass $M_{2}$ can be conveniently used for this purpose.

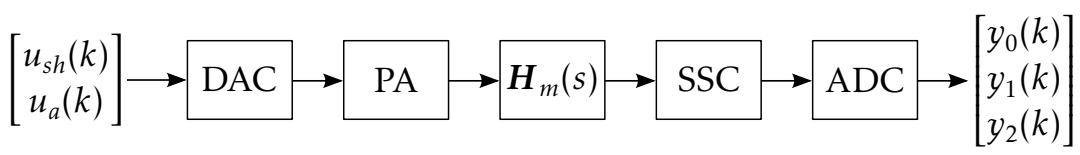

Figure 7.8: Block diagram showing the interconnection of the mechanical system and the electrical components

$D A C$ : DA-converters (dSPACE DS2102)

$P A$ : power amplifiers and actuator electronics

$\boldsymbol{H}_{m}(s)$ : mechanical system (including coupled 3D dynamics), cf. eqn. (7.1)

$S S C$ : sensor signal conditioning

$A D C: A D$-converters (dSPACE DS2004)

3 The dSPACE DA- and AD-converters use normalized signals, i.e. the signals are normalized by their respective full scale input/output ranges. As a result, $u_{s h}, u_{a}, y_{0}, y_{1}$ and $y_{2}$ are expressed in $[\mathrm{V} / \mathrm{V}]$. 
To illustrate this fact, recall the parametric model of the setup(s), shown in figure 7.5. Based on this model, Newton's second law of motion for the second machine mass is expressed by equation (7.3). Because the stiffness of the linear guidance flexures $k_{s_{2}}$ is much smaller than the structural stiffness $k_{2}$, it is easily recognized that the deformation $\Delta x(t) \equiv x_{2}(t)-x_{1}(t)$ can be approximated by scaling the acceleration $\ddot{x}_{2}(t)$ by a factor $-m_{2} / k_{2} .{ }^{4}$ For the experimental setups, this means that an acceleration level of $1 \mathrm{~mm} / \mathrm{s}^{2}$ for $\ddot{x}_{2}$ is roughly equivalent to an internal deformation $\Delta x$ of $-6.7 \mathrm{~nm}$.

$$
m_{2} \ddot{x}_{2}(t)=-k_{2}\left(x_{2}(t)-x_{1}(t)\right)-k_{s_{2}} x_{2}(t), \quad k_{s_{2}} \ll k_{2}
$$

The modelling of the sensors and actuators including their electronics has already been discussed in chapter 6. Specifically, the block diagram of the PEA channel is shown in figure 6.18. The voice coil actuator is operated in voltage-mode, corresponding to the block diagram of figure 6.22a. For both actuators, the dominant dynamic effect of the actuator electronics (apart from amplification) is the introduction of first-order low-pass dynamics in the transfer functions $Q(z), S(z)$ and $S_{2}(z)$. The output impedance of the PEA pre-amplifier and the capacitance of the PEA itself form a first-order low-pass RC-filter with its pole at $100 \mathrm{~Hz}$. The VCA coil impedance has a single pole at approximately $680 \mathrm{~Hz}$.

The electrodynamic shaker is also operated in voltage-mode, corresponding to the block diagram of figure 6.22a. However, due to the small coil inductance, it can be modelled as a simple gain in the frequency range of interest. The signal conditioning of the accelerometers is performed by a Brüel \& Kjær Nexus 2692 conditioning amplifier, which implements charge amplification, calibrated variable voltage gain and second order high-pass and low-pass (antialiasing) filters.

An overview of the relevant parameters for the electronic system components is presented in table A.2 in appendix A.2.2.

\subsubsection{Combined electromechanical model}

Combining the models of the actuator electronics, the sensor electronics and the mechanical system (including the coupled 3D dynamics) results in the overall electromechanical models for the PEA-based and VCA-based setups. In figure 7.9, the frequency responses of these combined models are shown for both setups.

4 Note that this method is only valid in the absence of any external forces on the second machine mass, and provides a quasi-stationary approximation (because damping effects are ignored). 

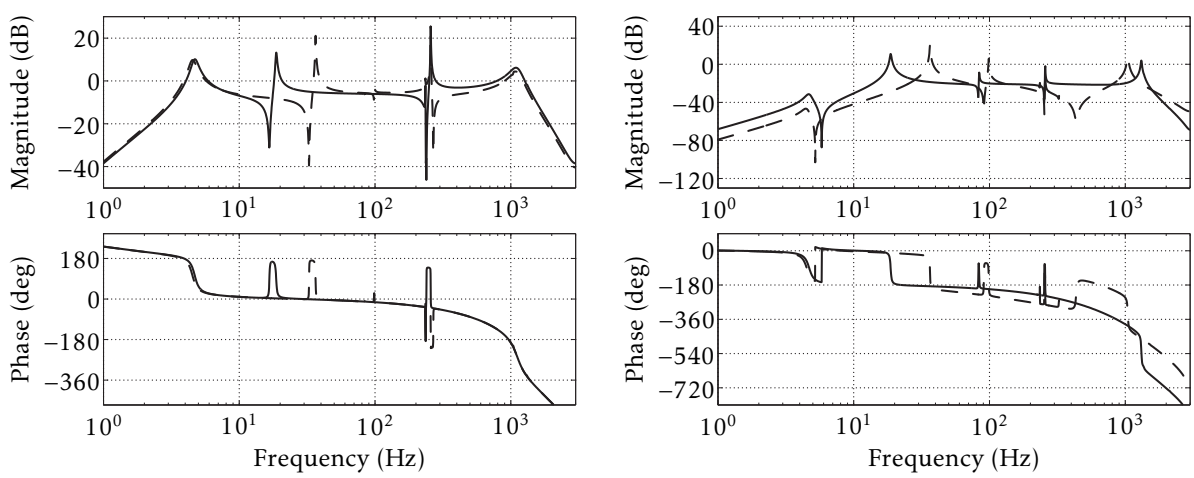

(a) $T(z)=Y_{0}(z) / U_{s h}(z)$

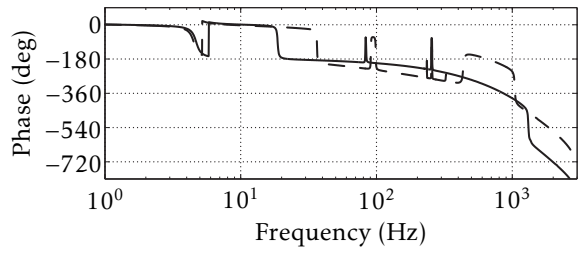

(b) $Q(z)=Y_{0}(z) / U_{a}(z)$
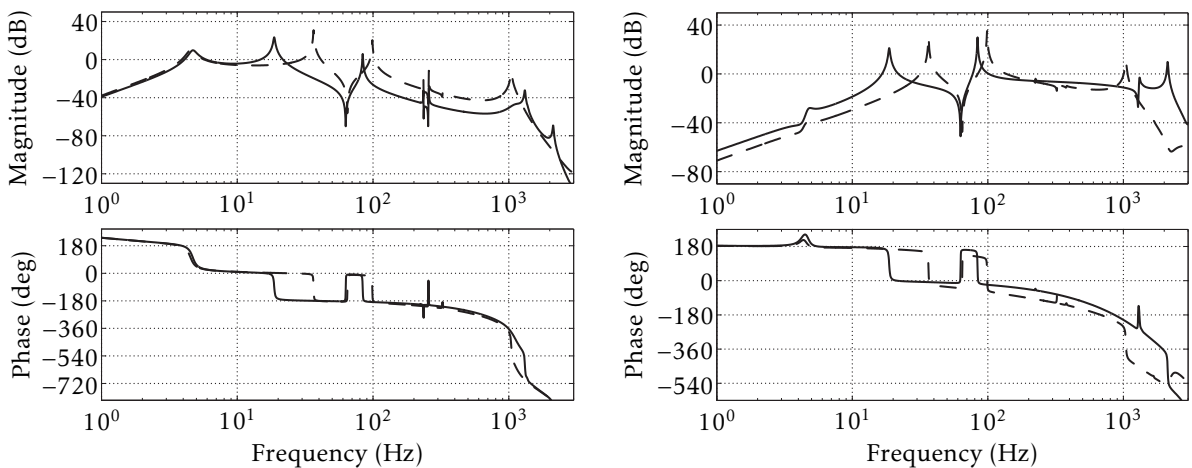

(c) $P(z)=Y_{1}(z) / U_{s h}(z)$

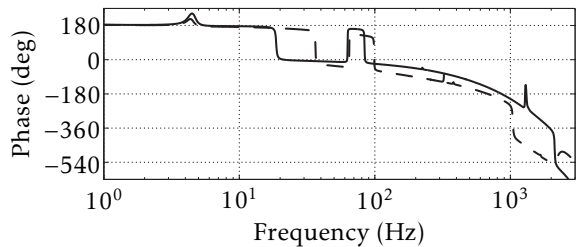

(d) $S(z)=Y_{1}(z) / U_{a}(z)$
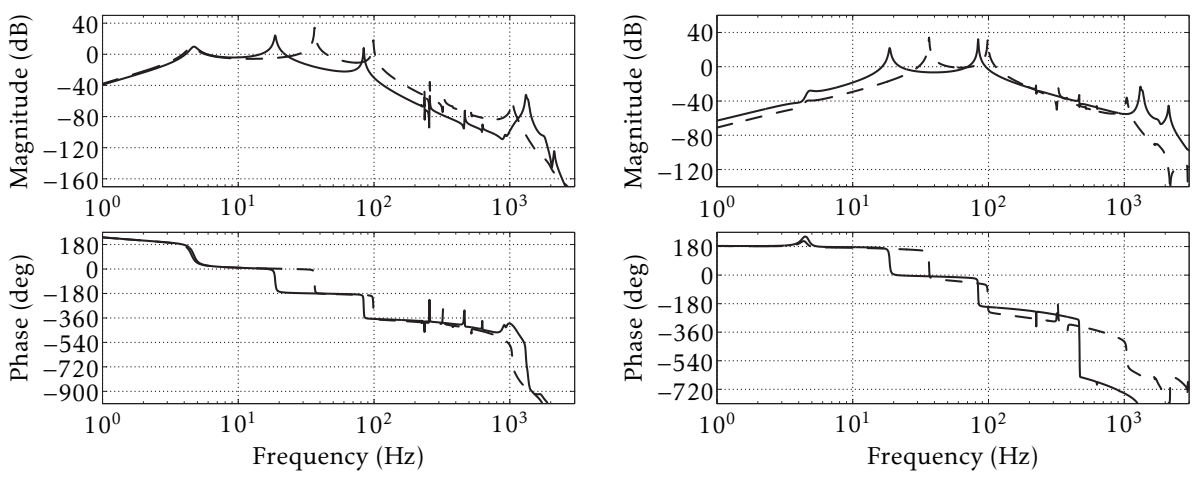

(e) $P_{2}(z)=Y_{2}(z) / U_{s h}(z)$

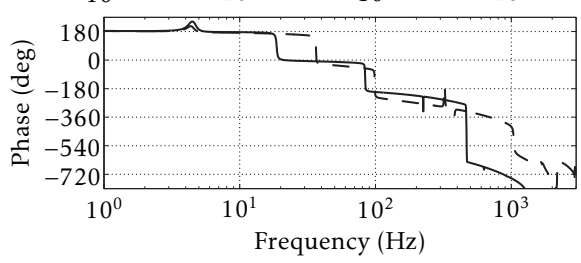

(f) $S_{2}(z)=Y_{2}(z) / U_{a}(z)$

Figure 7.9: Frequency response of the $3 D$ setup models including electronics — : setup with VCA; - - : setup with PEA 
The most important aspect of the electronics is the negative phase shift in all the frequency responses compared to figure 7.7. In the following chapter, it is shown that this negative phase shift is an important limiting factor for both the feedback control performance as well as the feedforward control performance.

This phase lag is particularly clear in the frequency range above $100 \mathrm{~Hz}$. It is caused primarily by the anti-aliasing filters in the sensor signal conditioning. Moreover, nearly a full sample delay is introduced due to the sequence of the AD- and DA-conversions in the real-time implementation, resulting in additional phase lag. ${ }^{5}$

\subsection{System identification}

Although the parametric models that have been presented in the previous sections offer insight into the dynamic behaviour of the setups, the model is most likely not sufficiently accurate to describe the actual dynamic behaviour accurately enough. Moreover, the parameter values that are used in the parametric models are rough estimates of the real values. As a result, the parametric models are not suited for the design of the feedback controller nor for application in the real-time implementation of the adaptive feedforward controller.

Therefore, system identification experiments are required to obtain accurate models of the setups. Specifically, accurate models of the secondary path $S(z)$ and the quaternary path $Q(z)$ are required for the real-time implementation of the adaptive feedforward controller (see e.g. figure F.2 in appendix F.1). The model of secondary path is also required to design the feedback controller. The remaining transfer functions $P(z), P_{2}(z), T(z)$ and $S_{2}(z)$ are also identified. These transfer functions are not required for the implementation or design of the controllers, but are used for simulations and prediction of the vibration isolation performance. ${ }^{6}$

In this thesis, a frequency domain subspace identification method is applied using periodic excitation signals. The entire system identification procedure is presented in detail in appendix E.

5 In the dSPACE real-time code, the AD-conversions are performed first and only then the DAconversions are executed.

6 Note that it is usually not possible to identify the transfer functions $P(z), P_{2}(z)$ and $T(z)$ in practical situations, as the user has no control over the external excitation source(s). 
The use of periodic excitations has two major advantages (among others) [Pintelon and Schoukens (2001), Ch. 2, 3 and 10]:

- The system response to the periodic signal is also periodic, whereas the noise contributions are generally not periodic. As a result, by averaging over several periods, the signal-to-noise ratio (SNR) is improved. The SNR of the data ultimately determines the model accuracy that can be achieved.

- For frequency domain identification methods, the data set can be confined to the excited frequencies only. For each excited frequency only 2 data points (e.g. amplitude and phase) need to be stored for each signal. This commonly results in a significant data reduction compared to the measured time traces.

The chosen periodic excitation signal is a random phase multisine signal, see equation (7.4). The user has extensive control over the excitation signal, i.e. the number of excited frequencies $N_{f}$, the base frequency $f_{b}$, the amplitude spectrum $A_{l}$ and the excited frequency grid $f_{l}$ are user-definable. The phases $\varphi_{l}$ are realizations of a zero mean random process that is uniformly distributed over $(-\pi, \pi)$.

$$
u_{M S}(t)=\sum_{l=1}^{N_{f}} A_{l} \cos \left(2 \pi f_{l} t+\varphi_{l}\right), \quad f_{l}=k_{l} f_{b}, \quad k_{l} \in \mathbb{N}
$$

A state-space model structure is chosen to describe the system dynamics. For the estimation of the state-space model parameters, a frequency domain subspace identification algorithm is used [McKelvey et al. (1996), Algorithm 2] (see also appendix E.5). The algorithm requires the user to select only one parameter $q$, which determines the size of the data matrices that are involved in the subspace identification. This parameter should be chosen larger than the (desired) system order $N_{x}$.

Given a chosen value for $q$, the identification algorithm can produce identified models of any order $N_{x} \leq q$ (note that $N_{x}$ is the selected system order, not the true system order). Unfortunately, the fit of the identified model to the measured frequency response data is not a smooth function of $q$ and $N_{x}$. Therefore, it is suggested to perform a numerical search over $q$ in the range $\left[1.5 N_{x}, 6 N_{x}\right]$, searching for the best least-squares fit to the measured frequency response [Pintelon and Schoukens (2001), Sect. 7.14].

However, obtaining the best least-squares fit is not necessarily the goal for the system identification. Other characteristics of the identified system may also be important but less suited to incorporate into the least-squares problem. For example, there is a risk of erroneously identifying minimum phase zeros as non-minimum phase (NMP) or vice versa. As discussed in chapters 4 
and 5, NMP zeros can have significant adverse effects on the performance of the feedback and feedforward control, and should therefore be identified correctly. Therefore, the identification of the zeros is checked manually by comparing the estimated non-parametric frequency response and the frequency response of the identified models.

In section 7.3.1, the system identification of the PEA-based setup is discussed. The identification experiments for the VCA-based setup and their results are described in section 7.3.2.

\subsubsection{PEA-based setup}

The sampling frequency $f_{s}$ is chosen at $3200 \mathrm{~Hz}$. This choice is a compromise between sufficient computational time (per sample) for the feedforward adaptation algorithm and sufficient bandwidth to prevent aliasing problems.

The base frequency $f_{b}$ of the multisine signal is set such that the excitation signal has a period of $2^{15}$ samples $\left(f_{b} \approx 0.10 \mathrm{~Hz}\right){ }^{7}$ The measured frequency data is averaged over 100 periods.

The setup is excited by a random phase multi-sine signal with $N_{f}=1786$ components. Up to $100 \mathrm{~Hz}$, all the higher harmonics of the base frequency are excited. From 100 to $1000 \mathrm{~Hz}$ a quasi-logarithmic grid with 600 frequency points per decade is used. Above $1000 \mathrm{~Hz}$, a quasi-logarithmic grid with 800 points per decade is used. By using quasi-logarithmic grids, the emphasis of the identification is shifted towards lower frequencies.

Three experiments are performed to collect the identification data. In the first experiment, the setup is only excited by the shaker. For this experiment, the amplitude spectrum is chosen to be constant over the excited frequency grid. In the second experiment, the setup is excited with the piezoelectric actuator. Again, the amplitude spectrum is flat over the excited frequency grid.

The low frequency signal-to-noise ratio of this second experiment is rather poor, as a result of the small gain of the PEA-related transfer functions $Q(z)$, $S(z)$ and $S_{2}(z)$ below $30 \mathrm{~Hz}$ (see figures 7.9b, 7.9d and 7.9f, dashed lines). Therefore, a third experiment (using the piezoelectric actuator) is performed. In this experiment, only the harmonics of the base frequency up to $10 \mathrm{~Hz}$ are excited, with an amplitude spectrum as shown in figure 7.10. The shape of the amplitude spectrum compensates somewhat for the reduced gain of the relevant transfer functions at low frequencies.

7 Using $2^{n}$ samples (with $n$ a positive integer) allows the use of the Fast Fourier Transform to compute the Discrete Fourier Transform. 


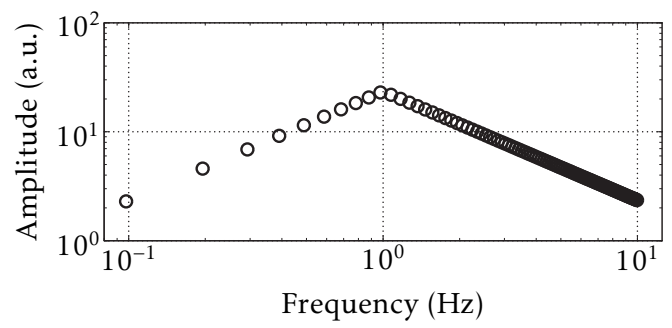

Figure 7.10: Amplitude spectrum for the third identification experiment for the PEA-based setup.

The frequency response data of the third experiment are used to replace the low frequency data of the second experiment. The combined data set is subsequently used in the identification procedure.

\section{Signal-to-noise ratios of the frequency response data}

In figure 7.11, the signal-to-noise ratio (SNR) is presented for the frequency response data that is used for the identification of the PEA-based setup. See appendix E.4.2 for details on the computation of the SNR (equation (E.14)).

From these figures, it is observed that the best SNR that is obtained in these experiments is typically 50-60 dB. Because the subspace identification algorithm puts more weight on frequency data with good SNR (see appendix E.5.2 for details), it can be expected that the identification results are less accurate in frequency ranges where the SNR is small.

Therefore, it can be concluded that the transfer functions $T(z), P(z)$ and $P_{2}(z)$ are difficult to identify at frequencies below approximately $1 \mathrm{~Hz}$. This is an inherent difficulty when force-to-acceleration transfer functions are identified for a system that does not allow rigid body motions. For such systems, the transfer functions have a low-frequency asymptote with a slope of +40 $\mathrm{dB} /$ decade, resulting in a poor SNR at low frequencies.

A similar conclusion is reached for the transfer functions $Q(z), S(z)$ and $S_{2}(z)$. Note that the SNR for these transfer functions in the frequency range between 1 and $10 \mathrm{~Hz}$ is nearly constant, as a result of the increased amplitude spectrum in this frequency range (recall figure 7.10). Otherwise, the SNR for these transfer functions would drop below $0 \mathrm{~dB}$ for $f \lesssim 5 \mathrm{~Hz}$.

Lastly, the transfer functions $P(z), P_{2}(z)$ and $S_{2}(z)$ may also prove difficult to identify above $200 \mathrm{~Hz}$ due to the high-frequency roll-off in these transfer functions. 


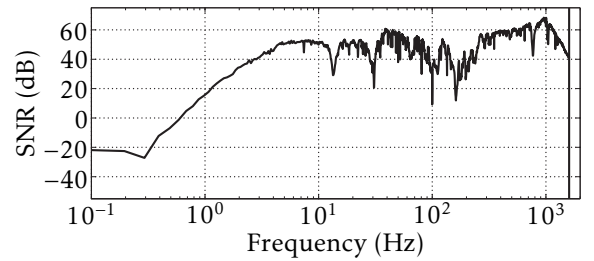

(a) $T(z)=Y_{0}(z) / U_{s h}(z)$

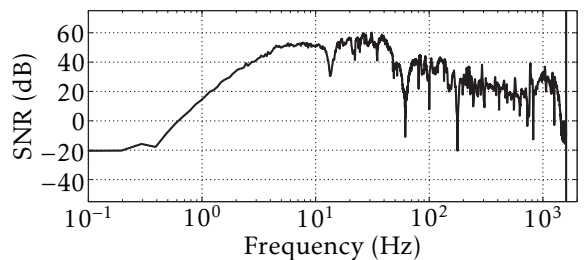

(c) $P(z)=Y_{1}(z) / U_{s h}(z)$

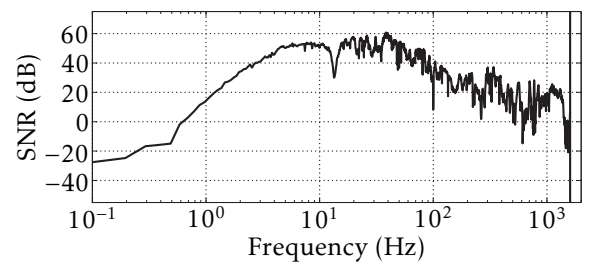

(e) $P_{2}(z)=Y_{2}(z) / U_{s h}(z)$

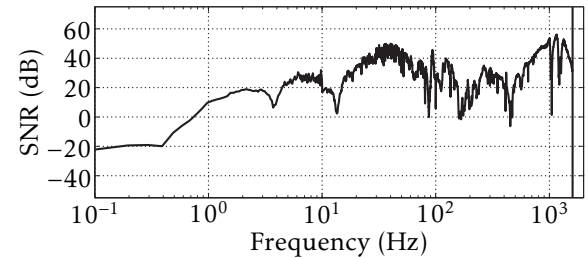

(b) $Q(z)=Y_{0}(z) / U_{a}(z)$

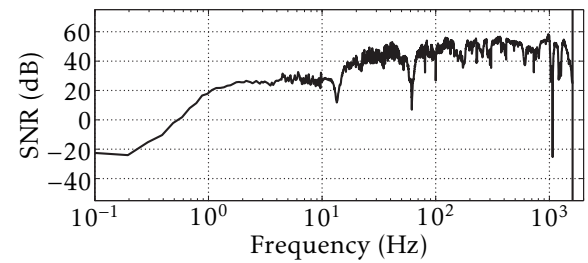

(d) $S(z)=Y_{1}(z) / U_{a}(z)$

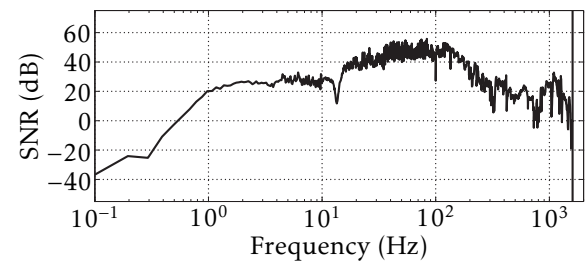

(f) $S_{2}(z)=Y_{2}(z) / U_{a}(z)$

Figure 7.11: Signal-to-noise ratios of the frequency data set used for the PEAbased setup identification

\section{Identified models of the PEA-based setup}

The transfer functions $T(z), P(z), P_{2}(z), Q(z), S(z)$ and $S_{2}(z)$ are identified separately from the frequency response data. Table 7.1 lists the values of the identification parameter $q$ and the selected system order $N_{x}$ for each of the transfer functions.

Table 7.1: Identification parameters $(q)$ and selected system orders $\left(N_{x}\right)$ for the identified PEA-based setup models

\begin{tabular}{|c|c|c|c|c|c|c|}
\hline & $T(z)$ & $P(z)$ & $P_{2}(z)$ & $Q(z)$ & $S(z)$ & $S_{2}(z)$ \\
\hline$q$ & 250 & 250 & 250 & 250 & 50 & 250 \\
\hline$N_{x}$ & 62 & 51 & 41 & 34 & 22 & 31 \\
\hline
\end{tabular}



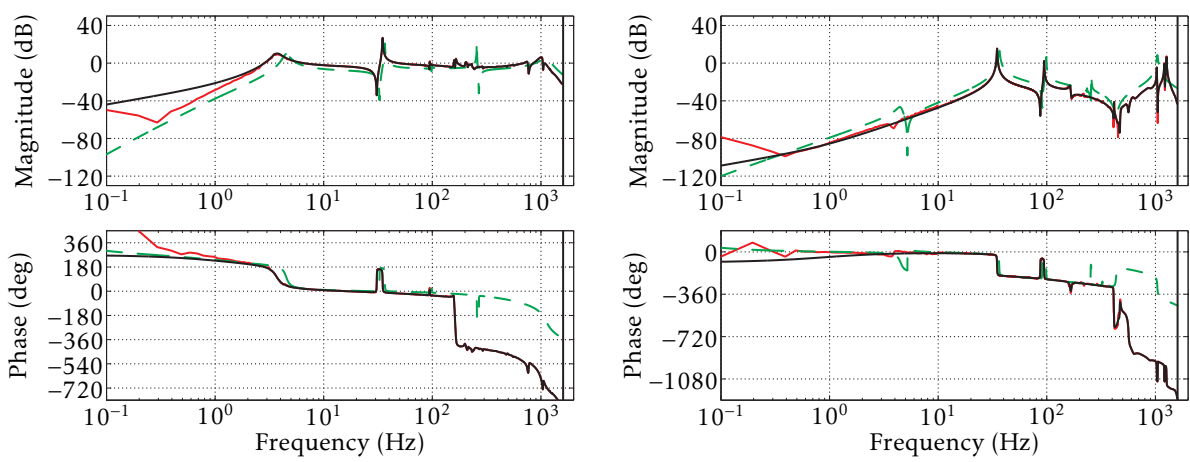

(a) $T(z)=Y_{0}(z) / U_{s h}(z)$

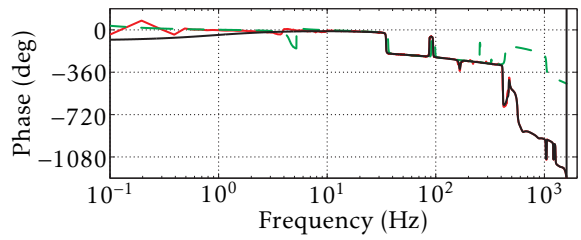

(b) $Q(z)=Y_{0}(z) / U_{a}(z)$
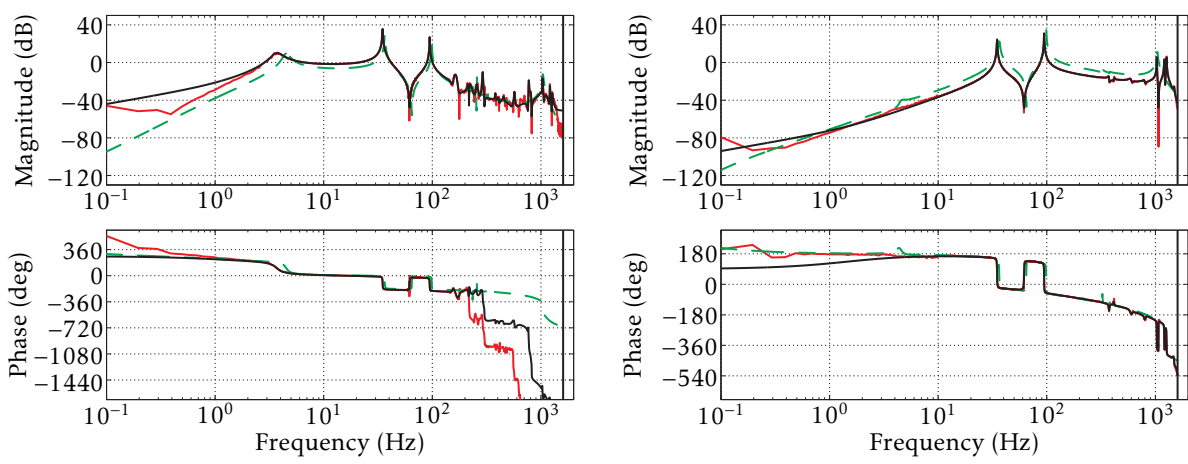

(c) $P(z)=Y_{1}(z) / U_{s h}(z)$

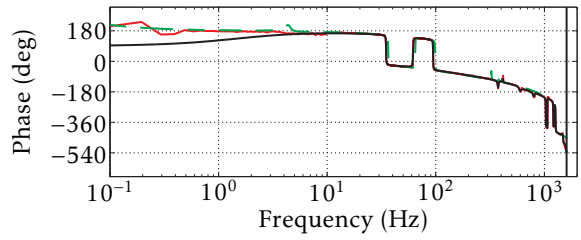

(d) $S(z)=Y_{1}(z) / U_{a}(z)$
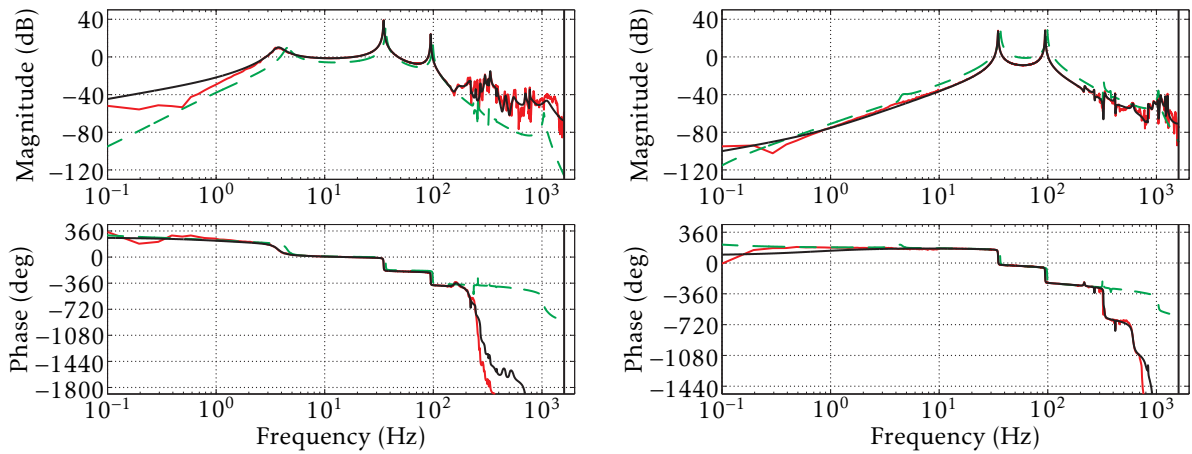

(e) $P_{2}(z)=Y_{2}(z) / U_{s h}(z)$

(f) $S_{2}(z)=Y_{2}(z) / U_{a}(z)$

Figure 7.12: Bode diagrams of the identified models for the PEA-based setup — : identified model, _ : non-parametric FRF, - - : parametric model 
In figure 7.12, the resulting identified model (black line) is compared to the non-parametric frequency response function (FRF, red line). The fit between the identified models and the non-parametric FRF is very good for all inputoutput channels, except in the frequency ranges with poor SNR (see figure 7.11). For comparison, the frequency response of the parametric model (green, dashed line) is also shown (see also figure 7.9).

The dominant resonance frequencies in the models are the floor mode at 3.7 $\mathrm{Hz}(\zeta=11 \%)$, the suspension mode at $35 \mathrm{~Hz}(\zeta=0.4 \%)$ and the structural mode at $95 \mathrm{~Hz}(\zeta=0.3 \%)$. It is concluded from the value of the suspension frequency that, compared to a soft mount system with a resonance frequency at $1 \mathrm{~Hz}$ (and the same supported mass), the hard mount in the PEA-based setup has a support stiffness that is approximately 1200 times higher.

Although the identified models have similar dynamic behaviour to the parametric models, several important differences are observed by comparing the identified model and the parametric model:

- The floor mode, suspension mode and (first) structural mode have shifted to lower frequencies. This is especially clear for the floor mode which has moved from 4.5 to $3.7 \mathrm{~Hz}$, see e.g. figure 7.12a. These shifts are likely due to an overestimation of the stiffness values in the parametric model.

- The gain of the identified models for $Q(z), S(z)$ and $S_{2}(z)$ is clearly smaller than predicted by the parametric model. This reduced gain is thought to be caused by the hysteresis in the piezoelectric actuator, see also figure 6.17 .

- In the identified model of the secondary path $S(z)$ (figure 7.12d) three poorly damped resonances are observed in the frequency range between 1000 and $1300 \mathrm{~Hz}$. Only one of these resonances is present in the parametric model. The other resonances are most likely internal resonances of the piezoelectric actuator.

- The tertiary path (figure 7.12a) has a non-minimum phase (NMP) zero pair at approximately $160 \mathrm{~Hz}$, which is associated with a tilting mode of the floor body $M_{0}$. This NMP zero pair can not be stably inverted by the (adaptive) feedforward controller. As a result, the vibration isolation performance at this frequency will be reduced.

- Many additional resonances can be observed above $200 \mathrm{~Hz}$, e.g. in the transfer functions $P(z), P_{2}(z)$ and $S_{2}(z)$. Some of these resonances are off-axis resonances of the major bodies (see also section 7.2.2 and figure 7.9). Others are resonances of the housings of the major bodies, the laboratory table and the base plate on which the setup is mounted. The latter resonances have not been included in the parametric model. 


\section{Implications for control}

In chapter 8 , these identified models are used to perform the control experiments. Therefore, the implications of some characteristics of the identified models for control design and implementation are discussed.

For the feedback control design, the secondary path $S(z)$ is relevant. The identified model closely matches the estimated non-parametric frequency response. Therefore, any feedback controller that results in a stable closed loop model will almost certainly also stabilize the actual system.

Note that the secondary path $S(z)$ still exhibits an alternating pole-zero pattern over a large frequency range, allowing the use of DVF-like feedback control. The combination of high-frequency resonances and the phase lag due to the anti-aliasing filters may be a limiting factor in the achievable bandwidth for the feedback controller. The lower gain (compared to the parametric model) is not an issue, it merely means that larger control signals are required to achieve the same performance.

For the adaptive feedforward controller, two transfer functions are relevant:

- Quaternary path $Q(z)$ :

This transfer path is used in the internal model correction (IMC) of the response of the floor acceleration to the feedforward compensation signal. Above $10 \mathrm{~Hz}$, the model closely matches the estimated non-parametric frequency response. However, the floor resonance mode is not correctly modelled, which is the result of the poor SNR at this frequency.

Recall from section 5.1.1 that especially at low frequencies the model of $Q(z)$ must be accurate because the feedforward controller gain is large at low frequencies. Alternatively, by reducing the low-frequency gain of the feedforward controller, the IMC loop may be stabilized. This can be achieved by proper choice of the regularization filter, the leakage factor and/or the noise shaping filter, see section 5.3.4.

- Secondary path $S(z)$ :

To allow stable adaptation of the controller weights, the secondary path should not have phase errors larger than $90^{\circ}$ in magnitude. The phase error only becomes that large at frequencies below 1 $\mathrm{Hz}$. Once more, the robustness in this frequency range can be improved by applying leakage and regularization of the secondary path, see section 5.3.3. 
The implementation of the Causal Wiener filter also requires the secondary and quaternary path models, but also the models for the primary path $P(z)$ and the tertiary path $T(z)$. Although the models of $S(z), T(z)$ and $Q(z)$ are quite accurate over most of the frequency range, the fit of the primary path model to the non-parametric frequency response is not so good at frequencies above $200 \mathrm{~Hz}$. The poor fit is caused by the poor SNR in this frequency range combined with the multitude of resonance modes that occur in this frequency range. The model errors in this frequency range may prove problematic.

\subsubsection{VCA-based setup}

For the identification of the VCA-based setup, the same multisine frequency grid is used. Only two experiments are performed (one using the shaker and one using the VCA). For both experiments, the amplitude spectrum is constant over the excited frequencies.

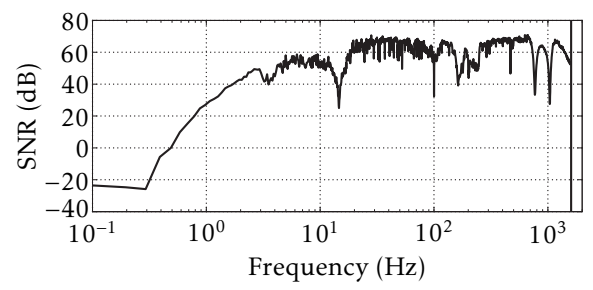

(a) $T(z)=Y_{0}(z) / U_{s h}(z)$

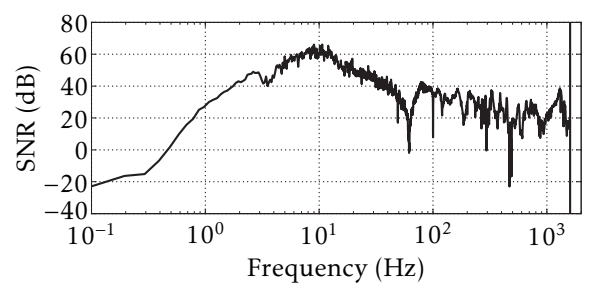

(c) $P(z)=Y_{1}(z) / U_{s h}(z)$

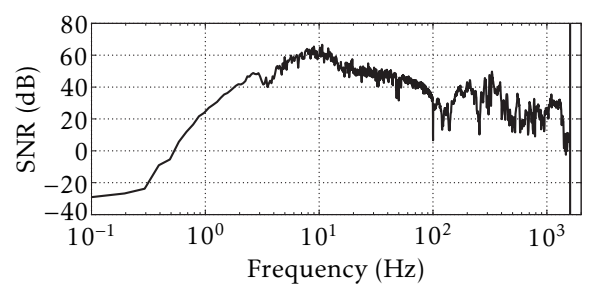

(e) $P_{2}(z)=Y_{2}(z) / U_{s h}(z)$

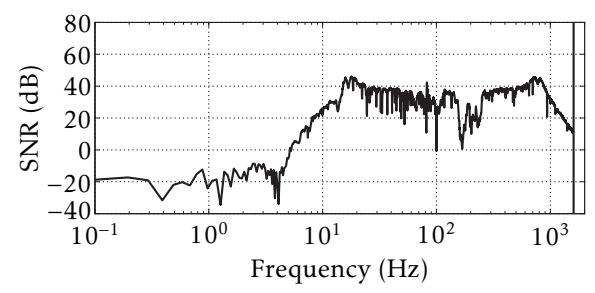

(b) $Q(z)=Y_{0}(z) / U_{a}(z)$

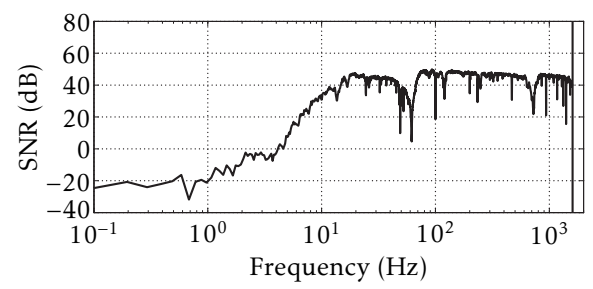

(d) $S(z)=Y_{1}(z) / U_{a}(z)$

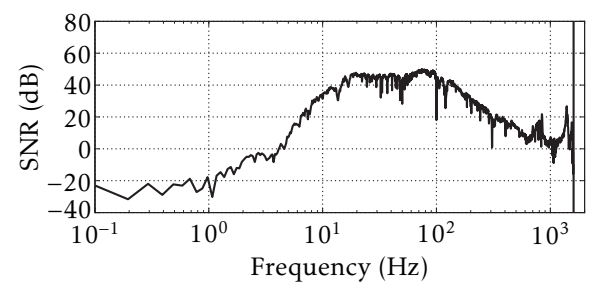

(f) $S_{2}(z)=Y_{2}(z) / U_{a}(z)$

Figure 7.13: SNR of the identification data set used for the VCA-based setup 


\section{Signal-to-noise ratios of the frequency response data}

Figure 7.13 shows the signal-to-noise ratios for the data set used for the identification of the VCA-based setup. A similar conclusion can be drawn as previously for the PEA-based setup: all transfer functions are difficult to identify at low frequencies $\left(f<2 \mathrm{~Hz}\right.$ for $T(z), P(z)$ and $S_{2}(z), f<10 \mathrm{~Hz}$ for $Q(z), S(z)$ and $S_{2}(z)$ ). Moreover, $P(z), P_{2}(z)$ and $S_{2}(z)$ are difficult to identify above 100 $\mathrm{Hz}$, due to the roll-off in these transfer functions.

\section{Identified models of the VCA-based setup}

For the VCA-based setup a single state-space system is identified with three outputs and two inputs. The search over $q$ and $N_{x}$ results in a good fit for $q=300$ and $N_{x}=72$. This initial system order is deemed too large. Therefore, nine complex-conjugate pole pairs and their (nearly) cancelling zeros have been removed manually, resulting in a $54^{\text {th }}$ order system.

In figure 7.14 , the identified model is compared to the non-parametric frequency response function and the parametric model of figure 7.9. Once more, the identified model corresponds very well to the non-parametric data in the frequency ranges with relatively large signal-to-noise ratios.

The floor mode is again observed at $3.7 \mathrm{~Hz}(\zeta=11 \%)$, the suspension mode has a frequency of $17 \mathrm{~Hz}(\zeta=1.6 \%)$ and the structural resonance occurs at 82 $\mathrm{Hz}(\zeta=0.3 \%)$. Compared to a soft mount system with a suspension frequency at $1 \mathrm{~Hz}$, the support stiffness of the VCA-based setup is approximately 300 times larger. Also, note that, the suspension mode has a four times larger damping ratio compared to the PEA-based setup. This is mostly caused by the back-emf damping in the voice coil actuator.

In comparison to the parametric model, several resonance modes have shifted to lower frequencies. This is especially true for the floor mode at $3.7 \mathrm{~Hz}$ (see e.g. figure 7.14a) and the internal resonance of the VCA module at $800 \mathrm{~Hz}$ (see figure $7.14 \mathrm{~b}$ ). Apparently, the stiffness of some components is not as high as assumed in the parametric model.

Moreover, the transfer function $T(z)$ has non-minimum phase zeros at approximately $160 \mathrm{~Hz}$ (see figure 7.14a), just like in the PEA-based setup. Therefore, it is expected that the adaptive feedforward controller does not achieve any vibration isolation performance at this frequency. 

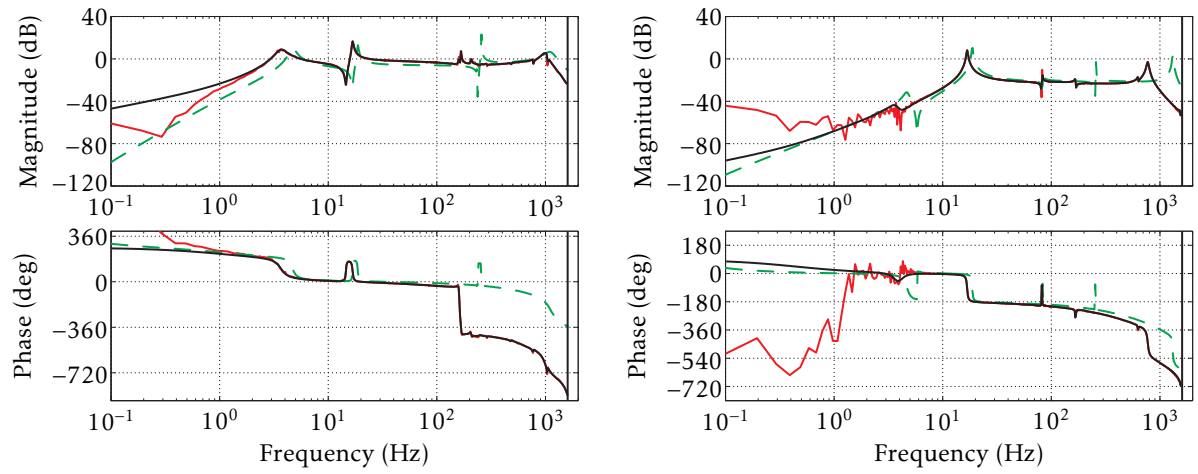

(a) $T(z)=Y_{0}(z) / U_{s h}(z)$

(b) $Q(z)=Y_{0}(z) / U_{a}(z)$

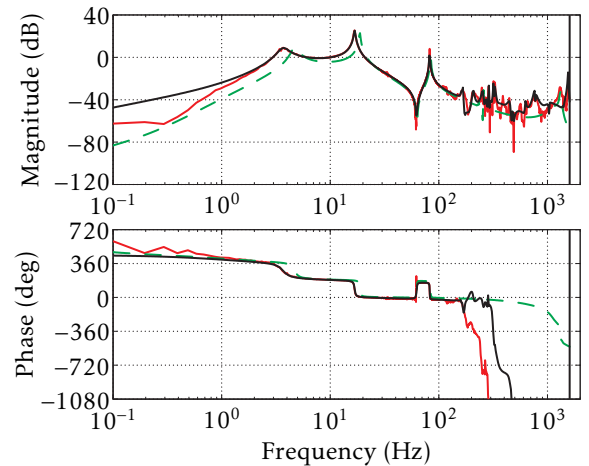

(c) $P(z)=Y_{1}(z) / U_{s h}(z)$
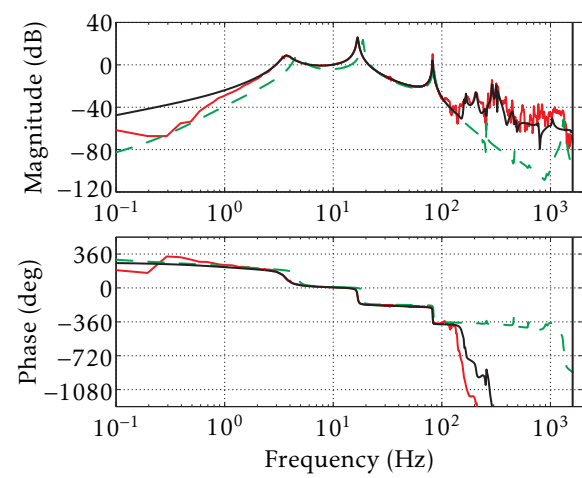

(e) $P_{2}(z)=Y_{2}(z) / U_{s h}(z)$
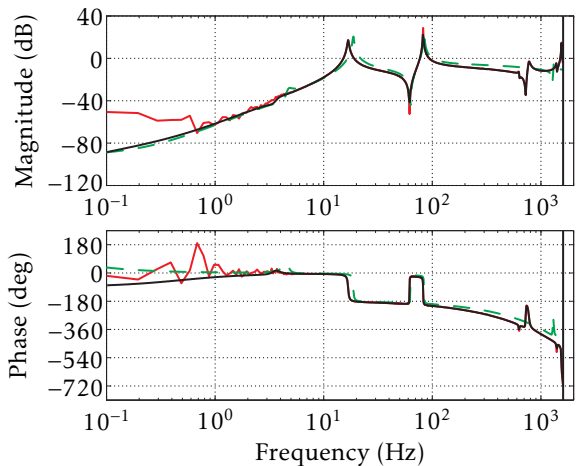

(d) $S(z)=Y_{1}(z) / U_{a}(z)$
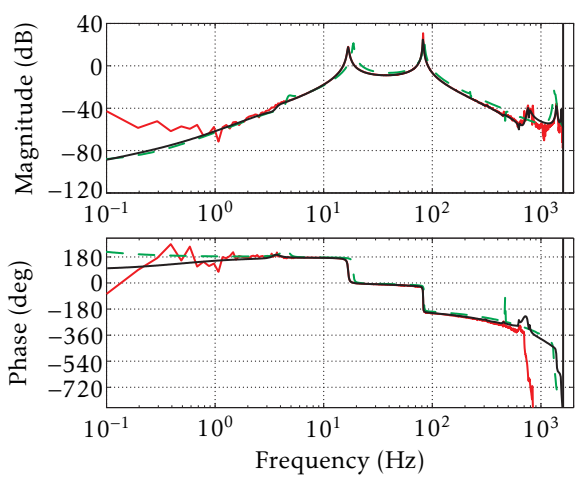

(f) $S_{2}(z)=Y_{2}(z) / U_{a}(z)$

Figure 7.14: Bode diagrams of the identified model for the VCA-based setup — : identified model, _— : non-parametric FRF, - - : parametric model 


\section{Implications for control}

The identified models are used for controller design and implementation in chapter 8 . Therefore, some characteristics of the identified models are discussed in anticipation of their use in the control experiments.

The secondary path $S(z)$ has an excellent fit to the non-parametric frequency response data. Moreover, the secondary path exhibits an alternating pole/zero pattern, meaning that the DVF-like feedback control can be applied. The phase lag at high frequencies, in combination with the resonance near 800 $\mathrm{Hz}$, may require some additional attention.

For the adaptive feedforward controller, the arguments used for the PEAbased setup can be repeated here. Although the models of the secondary path $S(z)$ and the quaternary path $Q(z)$ have some low frequency errors, it is expected that the robustness to these model errors can be improved sufficiently by regularization of the secondary path, applying leakage and using residual noise shaping.

Also for the Causal Wiener filter the arguments used for the PEA-based setup can be repeated for the VCA-based setup. The errors in the primary path model $P(z)$ may prove problematic.

\subsection{Recapitulation}

In this chapter, the experimental setup that is used for control algorithm testing has been presented. The setup has been designed with three well-defined resonance modes (floor, suspension and structural mode). The setup attempts to realize similar dynamic behaviour as the basic system model of section 2.4. This simplified model still describes many of the characteristics of typical vibration isolation problems that are encountered in precision equipment.

The setup is designed for predominant single-directional motion, in order to limit the complexity of the dynamic behaviour. However, due to the finite stiffness of the linear guidance mechanisms and alignment errors in the setup, some off-axis (e.g. tilt) resonances are excited and are observed at the accelerometers. A three-dimensional parametric model is constructed using the software package SPACAR to gain insight into this phenomenon. Moreover, the electronic components have been included into this model.

For feedback controller design and implementation of the adaptive feedforward controller more accurate models of the actual setup are required. These models have been obtained from system identification using multisine excitations, which offers the benefit of noise reduction by averaging over multiple measured periods. 
A frequency domain subspace identification algorithm is used to compute state-space models of the setup. The quality of the identified models is seen to be directly related to the signal-to-noise ratio of the identification data. In the frequency ranges with good signal-to-noise ratio, the identified models realize an excellent fit to the non-parametric frequency response data.

The implications of model errors on the controller design and implementation have been pointed out. Especially the model errors in the primary path $P(z)$ may prove troublesome for implementation of the Causal Wiener filter. No significant difficulties are expected for the feedback controller design and the adaptive feedforward filter implementation.

Both setups have a support stiffness that is much higher than the support stiffness of a soft mount system. Compared to a soft mount system with a suspension frequency of $1 \mathrm{~Hz}$, the support stiffness of the PEA-based and VCA-based setups are approximately 1200 and 300 times higher. 


\section{Chapter 8}

\section{Control experiments}

In this chapter, the results are presented for the control experiments that have been performed on the two experimental setups, which have been presented in the previous chapter. In these experiments, the feedback control methods of chapter 4 and the adaptive feedforward control methods of chapter 5 have been tested on either setup.

In section 8.1, the performance criteria are reviewed that are used for evaluating the various experiments. The results of the control experiments are treated separately for the PEA-based and VCA-based setups, in sections 8.2 and 8.3 respectively. Finally, the results for the two setups are compared and summarized in section 8.4 .

\subsection{Performance criteria}

The main (qualitative) objectives for an active hard mount vibration isolation system have been formulated in section 3.1 and are repeated below.
i. High support stiffness
ii. Small deformation transmissibility
iii. Skyhook damping of the suspension mode
iv. Improved damping of the relevant structural modes

Recall from the system identification results that have been presented in section 7.3, that the PEA-based and VCA-based setups have support stiffness values that are respectively 1200 and 300 times larger than the support stiffness of a soft mount system with a suspension frequency of $1 \mathrm{~Hz}$. It is deemed that these support stiffness values are sufficiently high. It then remains to be seen whether the other three objectives are met. 
In section 7.2.3 it has been argued that the acceleration signal $\ddot{x}_{2}(t)$ of the second machine body $M_{2}$ can be used as an approximate measure for the internal deformation. For the experimental setups, an acceleration level of 1 $\mathrm{mm} / \mathrm{s}^{2}$ for $\ddot{x}_{2}$ is roughly equivalent to a deformation $\Delta x$ of $-6.7 \mathrm{~nm}$. Therefore, in this chapter, the vibration isolation performance (in terms of the deformation transmissibility) is analysed using the measured acceleration signal $\ddot{x}_{2}(t)$.

The control system should achieve at least $40 \%$ skyhook damping of the suspension mode as well as $10 \%$ damping of the relevant structural modes. To evaluate the damping ratio improvement for the suspension and structural modes, the damping ratios of the identified (open loop) models are compared to the predicted damping ratios in the closed loop models. This estimate is deemed sufficiently accurate due to the excellent fit of the identified models to the measured data, see e.g. figures 7.12 and 7.14 .

Besides the performance criteria discussed above, which are derived directly from the stated main objectives, several other criteria are used to evaluate the control system performance:

- Acceleration $\ddot{x}_{1}$ of the first machine body $M_{1}$ :

This signal is the error signal used for feedback control as well as for the adaptation of the feedforward controller weights. It is therefore interesting to observe the relationship between the actual vibration isolation performance (the acceleration $\ddot{x}_{2}$ of $M_{2}$ ) and the observed error signal $\ddot{x}_{1}$.

- Control effort $u_{a}$ :

The control effort $u_{a}$ is also recognized as a relevant performance criterion. In this chapter, both RMS and peak-peak (pk-pk) measures are used to characterize the control effort.

- Turnaround time $T_{\text {turn }}$ :

To assess the computational complexity of the control algorithms, the turnaround time $T_{\text {turn }}$ is logged. The turnaround time is the total time required to execute all the real-time code in a single sample interval.

The machine acceleration signals $\ddot{x}_{1}$ and $\ddot{x}_{2}$ will be compared to the machine acceleration of the reference soft mount system that has been introduced in section 3.1. The reference transmissibility $T_{\text {ref }}(s)$ is repeated in equation (8.1), with $\omega_{\text {susp,ref }}=2 \pi \mathrm{rad} / \mathrm{s}, \zeta_{\text {susp,ref }}=0.70$ and $\epsilon_{\text {ref }}=1 \cdot 10^{-3}$. The reference machine acceleration level is obtained by filtering the measured floor acceleration signal through the transmissibility $T_{\text {ref }}(s)$.

$$
T_{\text {ref }}(s)=\frac{\omega_{\text {susp,ref }}^{2}}{s^{2}+2 \zeta_{\text {susp,ref }} \omega_{\text {susp,ref }} s+\omega_{\text {susp,ref }}^{2}}-\epsilon_{\text {ref }}
$$




\subsection{PEA-based setup}

In this section, the experimental results that have been obtained from the PEA-based setup are presented. In section 8.2.1, the chosen excitation and reference level for the performance evaluation are discussed. Then, the feedback control experiments are treated in section 8.2.2. Before discussing the adaptive feedforward control experiments in section 8.2.4, the Causal Wiener controller is used in section 8.2.3 to predict the achievable vibration isolation performance for the adaptive feedforward controller.

\subsubsection{Excitation level and target performance}

To allow a more realistic evaluation of the vibration isolation performance, the PEA-based setup is excited by the shaker such that the floor acceleration power spectrum is comparable in shape to the power spectrum of practical floors. This practical floor reference spectrum is based on the data presented in figure 2.2 and has been introduced in section 6.1.2. It is an extension of the VC-E vibration criterion and is therefore denoted by $\mathrm{VC}_{\mathrm{ext}}$ throughout this chapter.

The resulting acceleration power spectral density of the floor body $M_{0}$ is shown in figure 8.1a. The increased power spectral density at frequencies below $1 \mathrm{~Hz}$ is due to the $1 / f$-noise in the accelerometer.

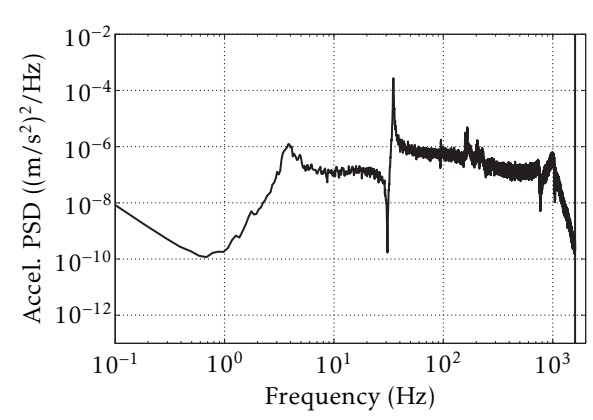

(a) Acceleration PSD

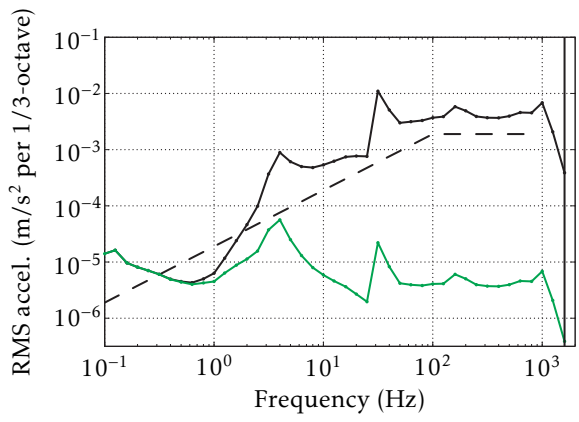

(b) RMS acceleration (per 1/3-octave)

Figure 8.1: Open loop floor $\left(M_{0}\right)$ acceleration level of the PEA-based setup

— : floor acceleration level of the experimental setup

- - : VC-E $E_{\text {ext }}$ floor acceleration level (fig. b)

— : reference machine acceleration level (fig. b) 
In figure $8.1 \mathrm{~b}$, the RMS floor acceleration level (black solid line) is compared to the VC- $\mathrm{E}_{\text {ext }}$ 1/3-octave RMS floor acceleration level (black dashed line). It can be concluded that these spectra roughly have the same shape, although the actual excitation on the setup has a higher level (by a factor of 3-5). The excitation level is chosen at this higher level to improve the signal-to-noise ratios for the various measurements. The total RMS $(0-1600 \mathrm{~Hz})$ level of the floor acceleration is $20 \mathrm{~mm} / \mathrm{s}^{2}$.

Moreover, the 1/3-octave RMS reference machine acceleration level is shown in the same figure (green solid line). It represents the residual acceleration level on the machine that would be achieved when the floor vibration transmissibility function is given by the idealized reference transmissibility function $T_{\text {ref }}(s)$, see equation (8.1). This reference machine acceleration level is obtained by filtering the measured floor acceleration level (black line) by the idealized transmissibility function $T_{\text {ref }}(s)$.

\subsubsection{Feedback control}

The primary purpose of the feedback controller is to increase the damping of the suspension mode and the structural mode. To this end, the feedback controller has been designed based on the DVF-like approach discussed in section 4.3. The frequency response of the feedback controller is shown in figure $8.2 \mathrm{a}$.

Because the feedback controller uses an acceleration measurement, the basic action to achieve damping is an integration of the measurement signal. However, the resulting high gain at low frequencies causes amplification of the
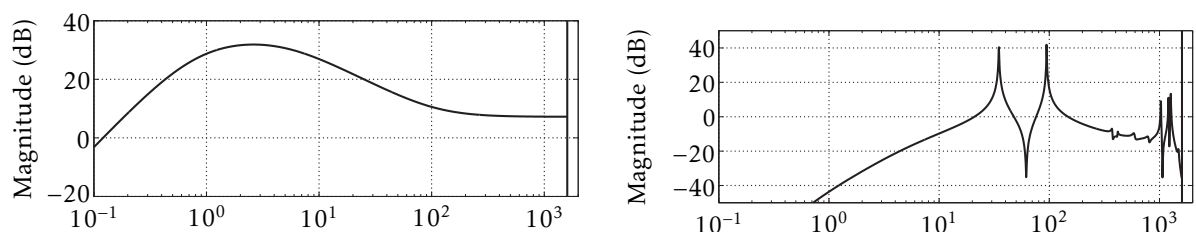

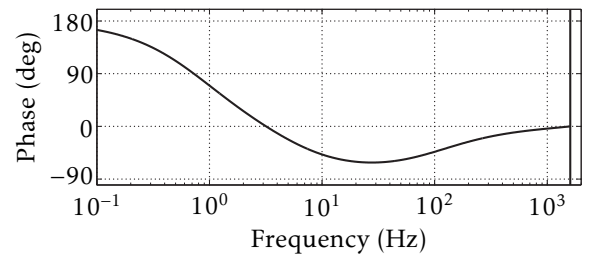

(a) Feedback controller

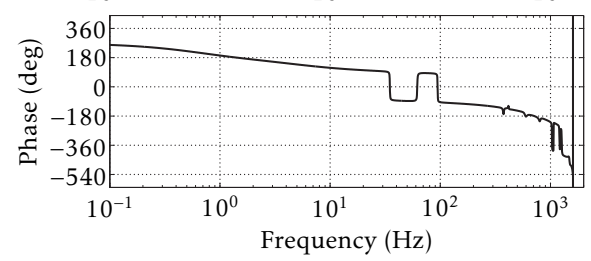

(b) Loop gain

Figure 8.2: Frequency response of the feedback controller (fig. a) and the resulting loop gain (fig. b) for the PEA-based setup. 
measurement noise and bias, resulting in large control signal drift. Therefore, a tame integrator with a pole at $5 \mathrm{~Hz}$ is used, as well as a second order high-pass filter (with real poles at 0.6 and $1 \mathrm{~Hz}$ ). Moreover, as discussed in section 7.2.3, the power amplifier and the piezoelectric actuator form an RCfilter with a pole at $110 \mathrm{~Hz}$. This pole is cancelled in the feedback controller by a zero. Finally, the gain has been tuned manually to achieve maximum damping for the structural mode.

The resulting loop gain that is formed by the feedback controller and the secondary path $S(z)$ is shown in figure $8.2 \mathrm{~b}$. There are four $0 \mathrm{~dB}$-crossings between 20 and $200 \mathrm{~Hz}$, which all have excellent phase margin (the smallest phase margin is $77^{\circ}$ at $148 \mathrm{~Hz}$ ).

Due to the undamped internal resonances in the hard mount, there are six more $0 \mathrm{~dB}$-crossings above $1 \mathrm{kHz}$. Fortunately, the loop gain has an alternating pole-zero pattern between $1 \mathrm{kHz}$ and $1.3 \mathrm{kHz}$. Moreover, the phase lag caused by the anti-aliasing filter is so large in this frequency range that the phase margin for these crossovers is at least $45^{\circ}$ (at $1.02 \mathrm{kHz}$ ), resulting in an overall stable closed loop response. The minimum gain margin is $3.2(10 \mathrm{~dB})$ at a frequency of $592 \mathrm{~Hz}$.

The reciprocal of the peak magnitude of the complementary sensitivity function is a measure of the largest relative model error that can be allowed for SISO systems [Franklin et al. (1994), Section 6.9.2]. For this feedback loop, the peak magnitude of the complementary sensitivity function is 1.3 at 1.02 $\mathrm{kHz}$. Therefore, relative model errors of at least $77 \%$ are allowed.

In table 8.1, the open and closed loop poles in the frequency range up to 200 $\mathrm{Hz}$ and their damping ratios are listed. In the open loop system, the floor resonance mode occurs at $3.7 \mathrm{~Hz}$ and the suspension mode and structural mode occur at $35 \mathrm{~Hz}$ and $95 \mathrm{~Hz}$, respectively. The latter resonance modes are poorly damped. The real pole at $110 \mathrm{~Hz}$ is the pole of the RC-circuit formed by the piezoelectric actuator and its amplifier.

Table 8.1: Open and closed loop poles of the PEA-based setup (0-200 Hz)

\begin{tabular}{|c|c|}
\hline \multicolumn{2}{|c|}{ Open loop } \\
\hline Freq. $(\mathrm{Hz})$ & $\zeta(\%)$ \\
\hline 3.7 & 11 \\
\hline 35 & 0.42 \\
\hline 95 & 0.28 \\
\hline 110 & (real) \\
\hline- & - \\
\hline- & - \\
\hline- & - \\
\hline
\end{tabular}

\begin{tabular}{|c|c|}
\hline \multicolumn{2}{|c|}{ Closed loop } \\
\hline Freq. (Hz) & $\zeta(\%)$ \\
\hline 0.6 & $($ real $)$ \\
\hline 1.1 & $($ real $)$ \\
\hline 3.7 & 11 \\
\hline 6.5 & $($ real $)$ \\
\hline 42 & 71 \\
\hline 74 & 37 \\
\hline 111 & $($ real $)$ \\
\hline
\end{tabular}


The feedback control has shifted the suspension mode to $42 \mathrm{~Hz}$ and has increased the damping ratio to $71 \%$. The structural mode occurs at $74 \mathrm{~Hz}$ in the closed loop system and has a damping ration of $37 \%$. It is concluded that the damping objectives (at least $40 \%$ suspension damping and at least $10 \%$ structural damping) are both met.

The closed loop structural damping ratio of $37 \%$ is much larger than the DVFprediction of $27 \%$, see equation (4.15) (resonance frequency at $95 \mathrm{~Hz}$, antiresonance frequency at $62 \mathrm{~Hz}$ ). The prediction is based on the assumption that the system can be approximated by a second order system. Moreover, the prediction is developed in the continuous-time domain. Therefore, the predicted value can only be used as a rough estimate. The larger than predicted value for the damping ratio is in accordance with [Vervoordeldonk et al. (2006)], where it is shown that higher damping values can be achieved by deviating from the pure DVF control approach.

\section{Results}

The closed loop performance is illustrated in the frequency domain in figure 8.3. The open loop and closed loop power spectral densities of the acceleration $\ddot{x}_{2}$ of the machine body $M_{2}$ are compared in figure 8.3a. A similar comparison for the acceleration $\ddot{x}_{1}$ is shown in figure $8.3 \mathrm{c}$. The beneficial effect of the increased damping of the suspension and structural modes is clear in both figures. Just like in the open loop case, the increased power spectral density below $1 \mathrm{~Hz}$ is caused by $1 / f$-noise in the sensors.

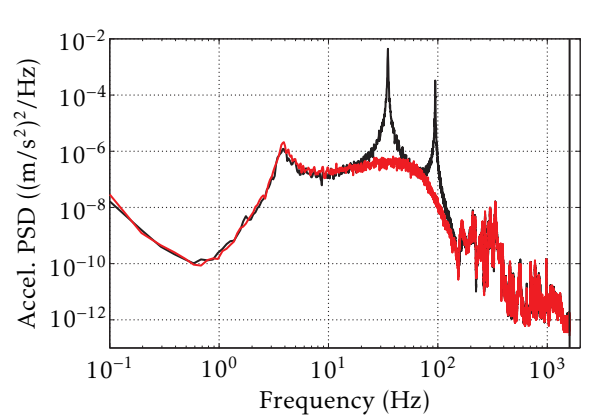

(a) Acceleration PSD of $M_{2}$

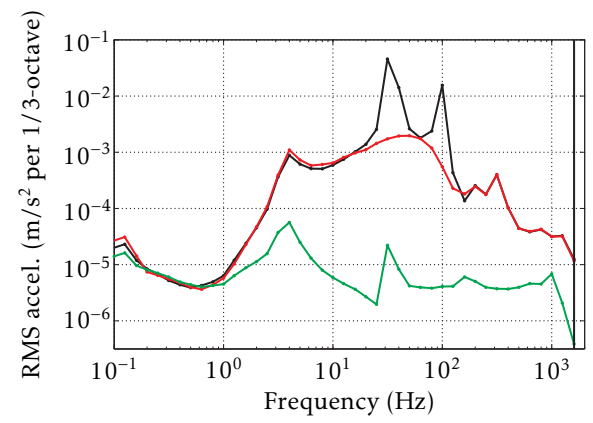

(b) RMS acceleration of $M_{2}$ (per 1/3-octave)

Figure 8.3: Comparison of open loop and closed loop machine acceleration levels of the PEA-based setup for the machine body $M_{2}$.

— : open loop acceleration level; _ _ closed loop acceleration level

— : reference machine acceleration level (fig. b) 


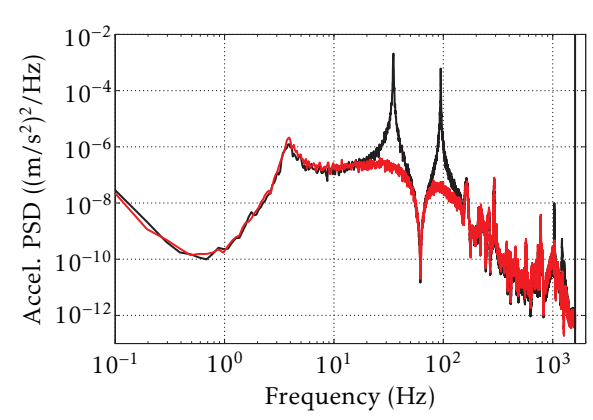

(c) Acceleration PSD of $M_{1}$

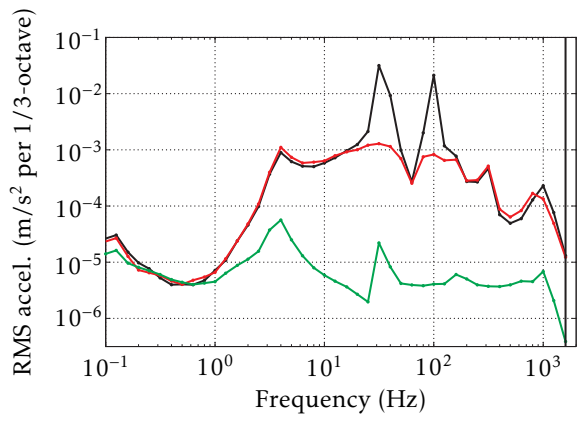

(d) RMS acceleration of $M_{1}$ (per 1/3-octave)

Figure 8.3 (cont.): Comparison of open loop and closed loop machine acceleration levels of the PEA-based setup for the machine body $M_{1}$.

— : open loop acceleration level; _ closed loop acceleration level

- : reference machine acceleration level (fig. $d$ )

In figures $8.3 \mathrm{~b}$ and $8.3 \mathrm{~d}$, the corresponding 1/3-octave RMS acceleration levels are presented. Moreover, the reference machine acceleration level is drawn for comparison. From these figures, it is clear that the closed loop acceleration levels are still much worse than the reference acceleration level.

This can also be concluded from the summary of the performance measures in table 8.2. The feedback controller has reduced the total RMS acceleration levels by approximately a factor ten $(-20 \mathrm{~dB})$. However, the acceleration levels are still much worse than the reference level, by at least a factor of $40(32 \mathrm{~dB})$.

Table 8.2: Performance measures for the PEA-based setup: Open loop (OL) and feedback controlled (FB)

\begin{tabular}{|c|c|c|c|}
\hline Measure & OL & FB & Unit \\
\hline${\text { reference }(\mathrm{RMS})^{\mathrm{a}}}^{\mathrm{a}}$ & 0.087 & 0.087 & $\mathrm{~mm} / \mathrm{s}^{2}$ \\
\hline$\ddot{x}_{0}(\mathrm{RMS})$ & 20 & 17 & $\mathrm{~mm} / \mathrm{s}^{2}$ \\
\hline$\ddot{x}_{1}(\mathrm{RMS})$ & 39 & 3.6 & $\mathrm{~mm} / \mathrm{s}^{2}$ \\
\hline$\ddot{x}_{2}(\mathrm{RMS})$ & 51 & 4.9 & $\mathrm{~mm} / \mathrm{s}^{2}$ \\
\hline$u_{a}($ RMS $)$ & - & 0.41 & $\mathrm{~V}$ \\
& & $(0.19)$ & $(\mathrm{N})$ \\
\hline$u_{a}($ pk-pk) & - & 3.1 & $\mathrm{~V}$ \\
& & $(1.5)$ & $(\mathrm{N})$ \\
\hline$T_{\text {turn }}$ & 20.4 & 20.6 & $\mu \mathrm{s}$ \\
\hline
\end{tabular}

a All RMS values are total RMS $(0-1600 \mathrm{~Hz})$ 
The required RMS control signal for the feedback controller is only $0.41 \mathrm{~V}$, with a corresponding peak-peak signal of $3.1 \mathrm{~V}$. The corresponding peak-peak force level is calculated as $60 \mathrm{~N}$ using the nominal PEA data (see table A.2). Because the piezoelectric actuator is placed in series with a compliant element, the (quasi-stationary) effective force level is only $1.5 \mathrm{~N}$, see equation (6.37) and table A.1. In section 6.2.1, the required effective force level is estimated to be only $0.14 \mathrm{~N}$ (peak-peak), i.e. a factor ten smaller. This difference is partly explained by the larger excitation level and partly by the hysteresis in the piezoelectric actuator that reduces its small-signal gain, see e.g. figure 6.17 .

Lastly, the required turnaround time is $20.6 \mu \mathrm{s}$, which amounts to only $6.6 \%$ of the sample interval and is only slightly more than the open loop turnaround time. Clearly, data acquisition, data logging and other housekeeping tasks are much more demanding than computing the feedback control signal.

\subsubsection{Causal Wiener filter}

In this section, a regularized Causal Wiener filter is computed with control effort weighing at low frequencies, see section 5.2.4. It is expected that lowfrequency noise on the reference sensor, combined with the large gain of the Causal Wiener filter, would otherwise lead to an excessively large peak actuator signal level.

The computation is based on the identified models from section 7.3.1. Note that closed loop transfer paths must be used, because the feedforward controller is used "on top of" the feedback controller of the previous section.

The frequency response of the regularization filter $S_{\text {reg }}(z)$ and the closed loop secondary path $S_{\mathrm{cl}}(z)$ are shown in figure 8.4a. Because the magnitude of the regularization filter is larger than the secondary path's magnitude below $2 \mathrm{~Hz}$, the gain of the regularized Causal Wiener filter must be reduced (compared to the standard Causal Wiener filter) in this frequency range.

From figure $8.4 \mathrm{~b}$, which shows the frequency response of the (standard) Causal Wiener filter and the regularized Causal Wiener filter, it is observed that this is indeed the case.

In the frequency range from approximately $4 \mathrm{~Hz}$ to $100 \mathrm{~Hz}$, the (regularized) Causal Wiener filter achieves a slope of roughly $-40 \mathrm{~dB} /$ decade, which means it basically performs a double integral action in this frequency range. From the phase plot, it is then concluded that the filter has negative sign. Physically, this means that the filter computes the floor displacement from the measured floor acceleration and generate a force proportional to this displacement, but with opposite sign. This means that the force due to the suspension stiffness is counteracted, which is exactly as expected from the discussion in section 2.6.2, see e.g. equation 2.36. 


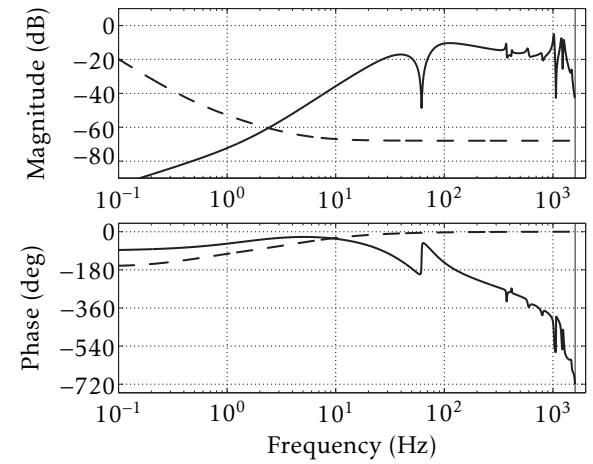

(a) Closed loop secondary path (-) and regularization filter (- -)
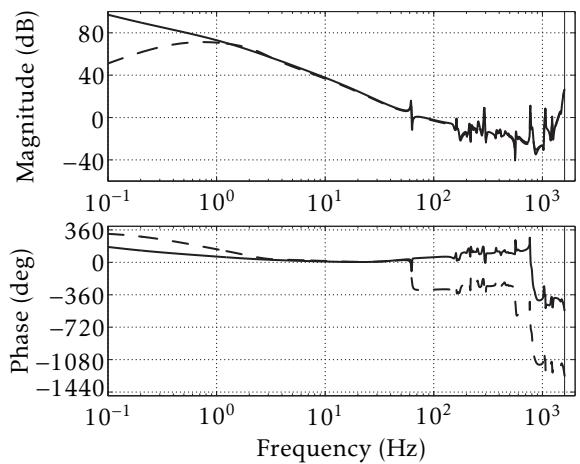

(b) Causal Wiener filter (-) and regularized Causal Wiener filter (- -)

Figure 8.4: Frequency responses of (a): secondary path (-) and regularization filter (- $)$, and (b): Causal Wiener filter (-) and regularized Causal Wiener filter (- -), for the PEA-based setup

At frequencies above $100 \mathrm{~Hz}$, the filter behaviour becomes much more complex. The filter is still attempting to counteract the forces due to the (dynamic) suspension stiffness. However, this suspension stiffness is no longer constant, but varies considerably with frequency.

Attempts to implement the regularized Causal Wiener filter have not been successful. It has not been possible to achieve any reduction of the acceleration levels. Apparently, the sensitivity of the overall performance to model errors is very large. In many cases, the system even becomes unstable. This is most likely caused by errors in the model of the closed loop quaternary path $\hat{Q}_{c l}(z)$, which is used in the internal model compensation (see section 5.1.1). The observed sensitivity and the resulting lack of performance for fixed gain feedforward control are compelling reasons to implement the feedforward controller using an adaptive algorithm.

\section{Prediction of adaptive feedforward performance}

Even though the regularized Causal Wiener filter can not be implemented, its model-based vibration isolation performance may be used to estimate the vibration isolation performance of the adaptive feedforward controller.

The vibration isolation performance for the regularized Causal Wiener filter is predicted from the identified model's frequency response to excitation by the shaker $\left(u_{s h}\right)$. In figure $8.5 \mathrm{a}$, the predicted performance in terms of the acceleration $\ddot{x}_{2}$ of the second machine mass $M_{2}$ is shown. Similar frequency 


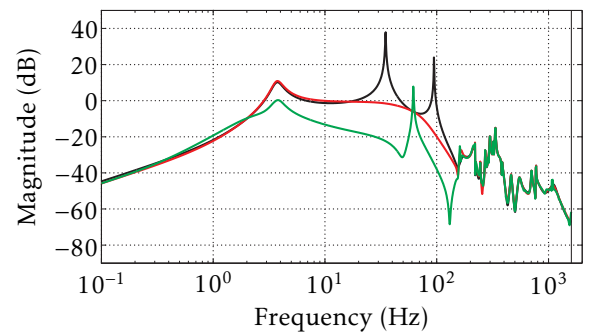

(a) $\ddot{X}_{2}(z) / U_{s h}(z)$

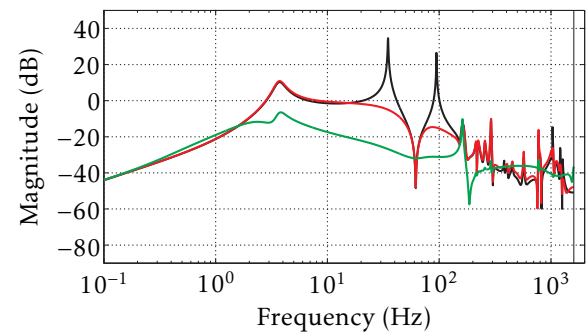

(b) $\ddot{X}_{1}(z) / U_{s h}(z)$

Figure 8.5: Frequency responses of (a): acceleration $\ddot{x}_{2}$ of machine mass $M_{2}$, and (b): acceleration $\ddot{x}_{1}$ of machine mass $M_{1}$, due to shaker excitation $u_{s h}$ for the PEA-based setup.

- : open loop — : feedback + regularized Causal Wiener filter

— : feedback only

responses are shown in figure $8.5 \mathrm{~b}$ for the acceleration $\ddot{x}_{1}$ of the first machine mass $M_{1}$. It is observed that the regularized Causal Wiener filter reduces the acceleration levels starting from approximately $3 \mathrm{~Hz}$, which is in accordance with the fact that the regularization becomes significant at frequencies below $3 \mathrm{~Hz}$, see figure $8.4 \mathrm{a}$.

From figure 8.5a, it is concluded that the feedforward compensation can significantly improve the feedback performance up to approximately $150 \mathrm{~Hz}$. At frequencies above $150 \mathrm{~Hz}$, the second mass $M_{2}$ is effectively decoupled from the first machine mass $M_{1}$ and the feedforward compensation no longer has any effect (just like the feedback control). Because the feedforward controller is designed to minimize the acceleration $\ddot{x}_{1}$ of the first machine mass $M_{1}$ (the available error signal), this decoupling is not compensated for. The important conclusion is that the vibration isolation performance is only improved over the frequency range in which the error signal (in this case $\ddot{x}_{1}$ ) and the actual performance signal (in this case $\ddot{x}_{2}$ ) have a very similar response to the disturbance sources.

At the anti-resonance frequency of the secondary path $(62 \mathrm{~Hz})$, the frequency response of $\ddot{x}_{2}$ is increased due to the sharp peak in the feedforward controller (see figure $8.4 \mathrm{~b}$ ). At this frequency, the control action has no effect on the observed error signal $\ddot{x}_{1}$ and is therefore not taken into account in the minimization. However, it is clear that the control action at this frequency does have an effect on other system responses. Therefore, it is advisable to cancel poorly damped zeros in the secondary (and tertiary) path by notch filters in the feedforward controller, to prevent any unchecked large control signals from deteriorating the system performance at these anti-resonance frequencies. 
Because the feedforward compensation is designed to minimize the error signal $\ddot{x}_{1}$, this acceleration level is reduced over a larger frequency range, see figure $8.5 \mathrm{~b}$. However, the improvement at frequencies above $200 \mathrm{~Hz}$ is only moderate due to the delay in the secondary and tertiary paths. Loosely speaking, the feedforward compensation reacts sluggish to the measured floor acceleration in this frequency range, resulting in a reduced performance.

Another notable feature in figure $8.5 \mathrm{~b}$ is that no improvement is obtained at approximately $160 \mathrm{~Hz}$. This is caused by an unstable zero pair in the tertiary path, see also figure 7.12a. This unstable complex-conjugate zero pair can not be compensated for, as it would require an unstable complex-conjugate pole pair in the feedforward controller. The unstable zero pair is caused by the fact that the reference accelerometer on the floor mass $M_{0}$ is mounted offaxis, thereby increasing the sensitivity to the tilting modes of the floor mass $M_{0}$. Therefore, unstable zeros in the secondary and tertiary paths (within the frequency range of interest) must be avoided as much as possible.

\subsubsection{Adaptive feedforward using FIR filters}

In this section, the experimental results that have been obtained using an adaptive feedforward controller with a FIR parametrization are presented. The results of two experiments are presented and compared to illustrate the achievable performance. These experiments are denoted by FIR1 and FIR2 respectively.

For both experiments, the feedforward controller is computed using an adaptive algorithm that combines the preconditioned FxLMS algorithm (see figure 5.8) with the modified FxLMS algorithm (see figure 5.9) to improve the convergence rate. Moreover, the residual noise shaping technique of section 5.3.4 is also implemented to shape the control signal in the frequency domain. Lastly, the adaptive algorithm uses the normalized LMS algorithm (see equation (5.48)) with leakage to update the controller weights. A full block diagram of the implemented adaptive feedforward controller is presented in appendix F.1.

\section{Parameter settings}

The controller parameters for each of the experiments are listed in table 8.3. Here, $L$ is the number of FIR parameters, $\bar{\mu}$ is the normalized step size (scale factor for the time-varying step size $\mu(k)), \epsilon$ is the regularization constant in the time-varying step size, $\gamma$ is the leakage factor and $\rho$ is the regularization constant that is applied to the closed loop secondary path (see also appendix F.1, equation (F.4)). 
Table 8.3: FIR feedforward controller parameters, PEA-based setup (see also appendix F.1)

\begin{tabular}{|c|c|c|c|c|c|}
\hline & $L$ & $\bar{\mu}$ & $\epsilon$ & $\gamma$ & $\rho$ \\
\hline FIR1 & 2800 & 0.1 & 0.1 & $1 \cdot 10^{-5}$ & $1.75 \cdot 10^{-4}$ \\
\hline FIR2 & 2400 & 0.1 & 0.1 & $1 \cdot 10^{-5}$ & $1.75 \cdot 10^{-4}$ \\
\hline
\end{tabular}

The two experiments not only differ by the number of implemented controller weights, but also in the choice of the noise shaping filter $N(z)$.

For the FIR1 experiment, the frequency responses of the noise shaping filter $N(z)$ and its complement $M(z)$ are shown in figure 8.6a. The noise shaping filter is designed to reduce the low-frequency content of the actuator signal. It has been designed with dominant poles at approximately $1.5 \mathrm{~Hz}$ and +40 $\mathrm{dB} /$ decade roll-on. The fast roll-on should ensure that the control signal is significantly reduced below the resonance frequency. Moreover, the complementary filter achieves $-40 \mathrm{~dB} /$ decade roll-off, which ensures that the highfrequency performance is influenced very little by the noise shaping filter. The peak gains of both filters are approximately 1.3, so the amplification near the resonance frequency is limited.

For the FIR2 experiment, the noise shaping filter is presented in figure 8.6b. The important difference is the fact that a notch filter is added at the antiresonance frequency of the secondary path at $62 \mathrm{~Hz}$. The purpose of the notch filter is to prevent the actuator from exciting the system at this frequency, which would otherwise result in an amplification of the acceleration level of the second machine mass $M_{2}$.
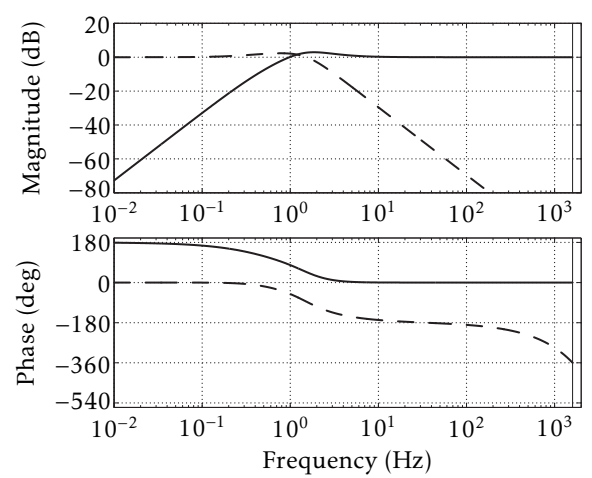

(a) FIR1 experiment
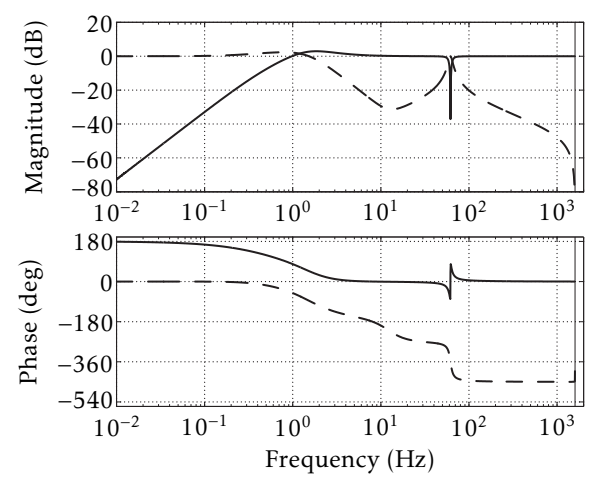

(b) FIR2 experiment

Figure 8.6: Frequency responses of the noise shaping filter $N(z)(-)$ and its complement $M(z)=1-N(z)(--)$. 


\section{Results: FIR1}

In figure 8.7, the results obtained with feedforward (FIR1) are compared to the case in which only feedback control is applied (see also section 8.3.2). The experimental results are primarily presented in the frequency domain, because the power spectral density plots and 1/3-octave RMS plots offer a better insight in the underlying dynamic behaviour than the time data.

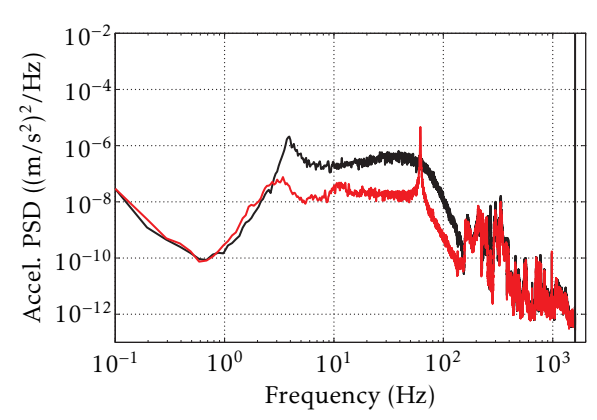

(a) Acceleration PSD of $M_{2}$

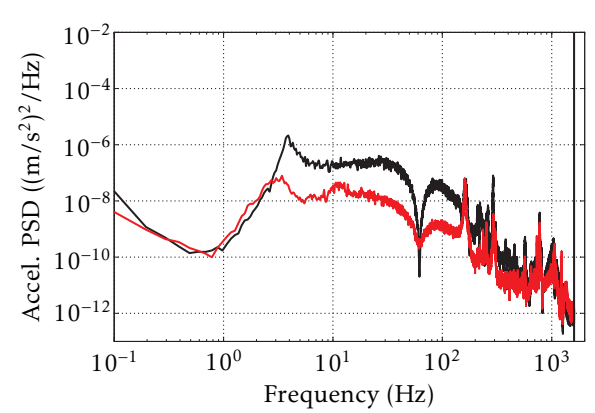

(c) Acceleration PSD of $M_{1}$

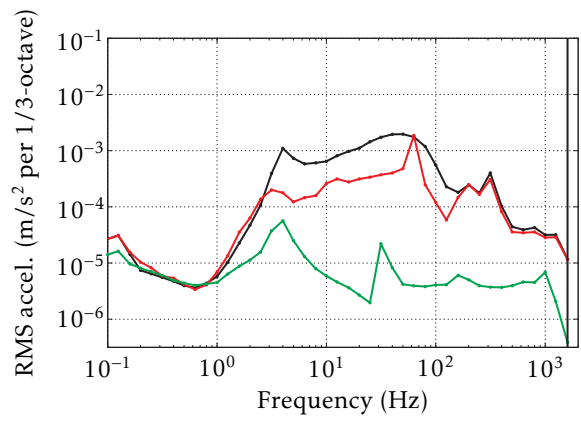

(b) RMS acceleration of $M_{2}$ (per 1/3-octave)

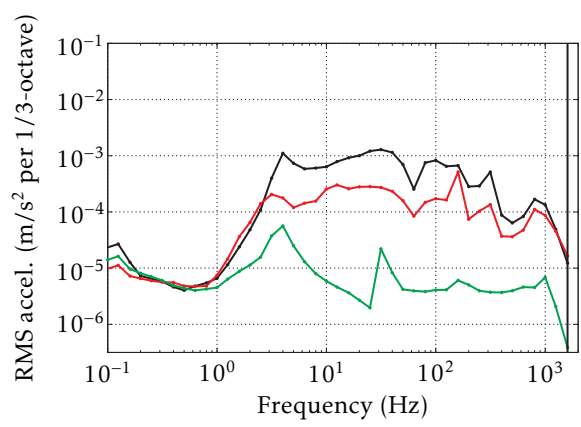

(d) RMS acceleration of $M_{1}$ (per 1/3-octave)

Figure 8.7: Comparison of closed loop and FIR feedforward compensated machine acceleration levels of the PEA-based setup

Fig. (a) and (b): machine mass $2\left(M_{2}\right)$, fig. (c) and (d): machine mass $1\left(M_{1}\right)$

— : closed loop acceleration level

— : FIR feedforward compensated acceleration level (FIR1)

- : reference machine acceleration level (fig. $b$ and $d$ ) 
At a glance, the power spectral density plots (figs. 8.7a and 8.7c) show a very similar behaviour as the Causal Wiener prediction (see figure 8.5), although the reduction of the acceleration levels is not as good, due to the following reasons:

\section{- Limited number of controller weights:}

Recall from section 5.3.5 that the FIR weights effectively describe the impulse response of the feedforward controller. The $1 \%$ settling time of the regularized Causal Wiener filter is found to be 2.8 seconds. With the chosen sample frequency of $3200 \mathrm{~Hz}$, the FIR parametrization would require more than 8800 coefficients to fully describe this impulse response. Obviously, by truncating the impulse response (to 2800 weights in this case), the vibration isolation performance will deteriorate.

- (Nearly-) uncontrollable modes:

Several of the resonances modes in the frequency range above 200 $\mathrm{Hz}$ are actually tilting modes that are observed by the accelerometer on the first machine mass $M_{1}$, but are nearly uncontrollable by the piezoelectric actuator (note that these modes do not appear in the secondary path, see figure 7.12c). This structural property of the underlying system is not considered by the Wiener filter problem.

In figure 8.7a, the amplification of the acceleration PSD is observed at the anti-resonance at $62 \mathrm{~Hz}$, exactly as predicted by the analysis using the regularized Causal Wiener filter. From the 1/3-octave RMS plot in figure $8.7 \mathrm{~b}$, it is observed that the dominant contribution to the acceleration of $M_{2}$ is made at this frequency.

In figure $8.7 \mathrm{c}$, the predicted effect of the unstable zero pair in the tertiary path at $160 \mathrm{~Hz}$ is also clearly observed. The residual motion at this frequency is the largest contribution to the total RMS acceleration of the first machine mass, see figure $8.7 \mathrm{~d}$.

Note that neither of these contributions can be compensated for by the feedforward controller due to the structure of the control setup (anti-resonance in the secondary path, unstable zero pair in the tertiary path).

The total RMS acceleration levels are listed in table 8.4, together with the RMS and peak-peak control signal level and the turnaround time.

The FIR feedforward controller has reduced the acceleration levels by a factor of 2-3 compared to the feedback controlled situation. However, the reference level is still a factor 15 to 30 smaller. As mentioned previously, the acceleration levels are mostly determined by frequency components that can not be reduced any further by the feedforward controller. Even if this were not the case, it is not possible to increase the performance any further by including more controller weights. The turnaround time has increased more than tenfold and now amounts to almost $95 \%$ of the sample interval. 
Table 8.4: Performance measures for the PEA-based setup: Open loop (OL), feedback controlled (FB) and feedforward compensated (FF FIR1, $L=2800$ )

\begin{tabular}{|c|c|c|c|c|}
\hline Measure & OL & FB & $\begin{array}{c}\text { FF FIR1 } \\
(L=2800)\end{array}$ & Unit \\
\hline reference (RMS) $^{\mathrm{a}}$ & 0.087 & 0.087 & 0.087 & $\mathrm{~mm} / \mathrm{s}^{2}$ \\
\hline$\ddot{x}_{0}(\mathrm{RMS})$ & 20 & 17 & 13 & $\mathrm{~mm} / \mathrm{s}^{2}$ \\
\hline$\ddot{x}_{1}(\mathrm{RMS})$ & 39 & 3.6 & 1.1 & $\mathrm{~mm} / \mathrm{s}^{2}$ \\
\hline$\ddot{x}_{2}(\mathrm{RMS})$ & 51 & 4.9 & 2.2 & $\mathrm{~mm} / \mathrm{s}^{2}$ \\
\hline$u_{a}(\mathrm{RMS})$ & - & 0.41 & 2.0 & $\mathrm{~V}$ \\
\hline$u_{a}(\mathrm{pk}-\mathrm{pk})$ & - & 3.1 & 15 & $\mathrm{~V}$ \\
\hline$T_{\text {turn }}$ & 20.4 & 20.6 & 293 & $\mu \mathrm{s}$ \\
\hline
\end{tabular}

a All RMS values are total RMS $(0-1600 \mathrm{~Hz})$

The required peak-peak control signal has increased to $15 \mathrm{~V}(75 \%$ of the full DA-range). This is the reason why the poles of the noise shaping filter have been designed at $1.5 \mathrm{~Hz}$. Moving the poles to a lower frequency would increase the control signal too much, leading to clipping of the DA-converter.

\section{Results: FIR2}

In this section, the results for the FIR2 experiment are presented. In this experiment, the noise shaping filter has been augmented with a notch at the anti-resonance frequency $(62 \mathrm{~Hz})$ in the secondary path, see figure $8.6 \mathrm{~b}$. This notch prevents the controller from exciting the second machine mass $M_{2}$ at this frequency. Recall from the FIR1 experimental results, that the total RMS acceleration level $\ddot{x}_{2}$ is dominated there by the motion at this particular frequency.

The PSD and 1/3-octave RMS plots for the FIR2 experiment are shown in figure 8.8. By comparing figures 8.7a and 8.8a, it is clear that the notch filter has removed the spike in the PSD spectrum of $\ddot{x}_{2}$. The PSD level at this frequency is now completely determined by the feedback performance. However, from the RMS plot in figure $8.8 \mathrm{~b}$, it must be concluded that the overall acceleration level is still dominated by this frequency component.

The performance at other frequencies is not significantly influenced by the addition of the notch filter, even though the number of controller weights $L$ has been reduced simultaneously from 2800 to 2400 . 


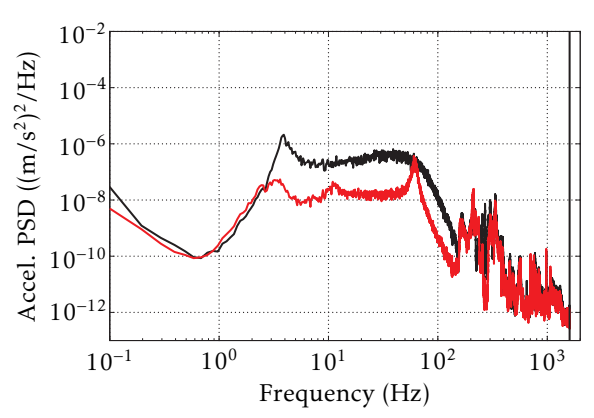

(a) Acceleration PSD of $M_{2}$

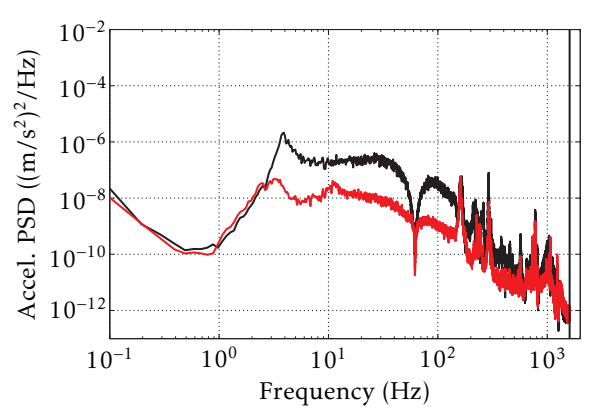

(c) Acceleration PSD of $M_{1}$

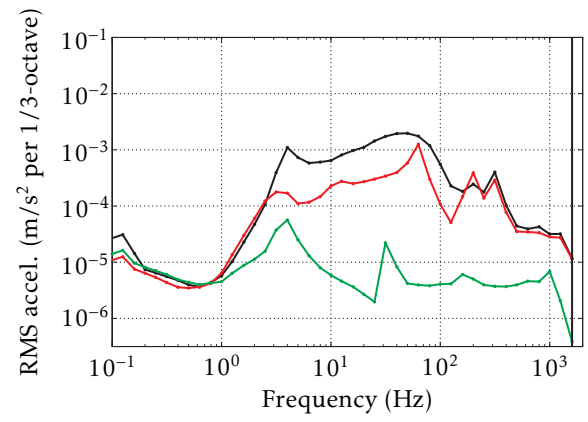

(b) RMS acceleration of $M_{2}$ (per 1/3-octave)

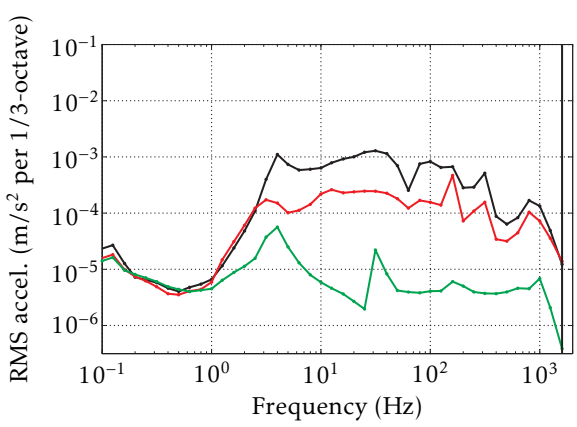

(d) RMS acceleration of $M_{1}$ (per 1/3-octave)

Figure 8.8: Comparison of closed loop and FIR feedforward compensated (with notch filter) machine acceleration levels of the PEA-based setup Fig. (a) and (b): machine mass $2\left(M_{2}\right)$, fig. (c) and (d): machine mass $1\left(M_{1}\right)$

- : closed loop acceleration level

_ : FIR feedforward compensated acceleration level (FIR2)

- : reference machine acceleration level (fig. $b$ and $d$ )

In table 8.5, a summary of the experimental results is presented once more, including the FIR2 experimental results. The total RMS level of $\ddot{x}_{2}$ has been reduced by approximately $20 \%$ by adding the notch filter. Note that the control signal levels have not changed significantly and that the turnaround time has even decreased by $40 \mu$ s due to the smaller number of controller weights. Still, the residual acceleration levels are an order of magnitude larger than the desired target level. Moreover, it is concluded that the vibration isolation performance can not be improved any further by this feedforward controller. Instead, changes have to be made to the system to remove the current bottlenecks in the performance. 
Table 8.5: Performance measures for the PEA-based setup: Open loop (OL), feedback controlled (FB) and feedforward compensated (FF FIR1 and FF FIR2)

\begin{tabular}{|c|c|c|c|c|c|}
\hline Measure & OL & FB & $\begin{array}{c}\text { FF FIR1 } \\
(L=2800) \\
(\text { no notch) }\end{array}$ & $\begin{array}{c}\text { FF FIR2 } \\
(L=2400) \\
(\text { notch })\end{array}$ & Unit \\
\hline ref. (RMS) & 0.087 & 0.087 & 0.087 & 0.087 & $\mathrm{~mm} / \mathrm{s}^{2}$ \\
\hline$\ddot{x}_{0}$ (RMS) & 20 & 17 & 13 & 13 & $\mathrm{~mm} / \mathrm{s}^{2}$ \\
\hline$\ddot{x}_{1}$ (RMS) & 39 & 3.6 & 1.1 & 0.96 & $\mathrm{~mm} / \mathrm{s}^{2}$ \\
\hline$\ddot{x}_{2}$ (RMS) & 51 & 4.9 & 2.2 & 1.7 & $\mathrm{~mm} / \mathrm{s}^{2}$ \\
\hline$u_{a}$ (RMS) & - & 0.41 & 2.0 & 1.9 & $\mathrm{~V}$ \\
$(0.19)$ & $(0.94)$ & $(0.89)$ & $(\mathrm{N})$ \\
\hline$u_{a}$ (pk-pk) & - & 3.1 & 15 & 15.4 & $\mathrm{~V}$ \\
\hline$T_{\text {turn }}$ & 20.4 & 20.6 & 293 & 252 & $\mu \mathrm{s}$ \\
\hline
\end{tabular}

a All RMS values are total RMS $(0-1600 \mathrm{~Hz})$

\subsection{VCA-based setup}

In this section, the experimental results that have been obtained from the VCA-based setup are presented. In section 8.3.1, the chosen excitation and reference level for the performance evaluation are discussed. Then, the feedback control experiments are treated in section 8.3.2.

Before discussing the adaptive feedforward control experiments in sections 8.3.4 and 8.3.5, the Causal Wiener controller is used in section 8.3.3 to predict the achievable vibration isolation performance for the adaptive feedforward controller.

\subsubsection{Excitation level and target performance}

The VCA-based setup is excited with the same excitation signal that has been used in the experiments that have been performed on the PEA-based setup. The resulting acceleration power spectral density of the floor body $M_{0}$ is shown in figure 8.9a. Clearly, the excitation is very similar in level and frequency content compared to PEA-based experiments (see also figure 8.1). The total RMS $(0-1600 \mathrm{~Hz})$ floor acceleration level is $17 \mathrm{~mm} / \mathrm{s}^{2}$. This value is only $85 \%$ of the RMS floor acceleration level of the PEA-based setup. The difference is caused by the improved damping of the suspension mode (compare figures $8.1 \mathrm{~b}$ and $8.9 \mathrm{~b})$. 


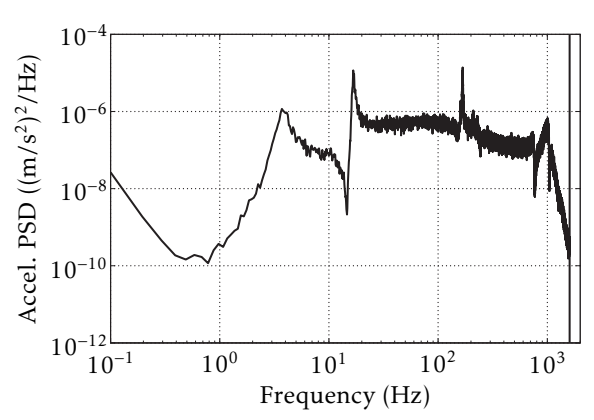

(a) Acceleration PSD

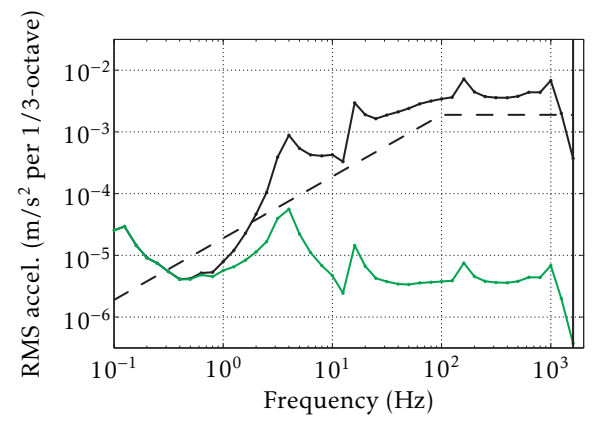

(b) RMS acceleration (per 1/3-octave)

Figure 8.9: Open loop floor $\left(M_{0}\right)$ acceleration level of the VCA-based setup

- : floor acceleration level of the experimental setup

- - : VC- $E_{\text {ext }}$ floor acceleration level (fig. b)

— : reference machine acceleration level (fig. b)

In figure $8.9 \mathrm{~b}$, the $1 / 3$-octave RMS floor acceleration level (black solid line) is compared to the VC- $\mathrm{E}_{\text {ext }} 1 / 3$-octave RMS floor acceleration level (black dashed line). The reference acceleration level that is used to evaluate the vibration isolation performance is also shown (green solid line).

\subsubsection{Feedback control}

The feedback controller for the VCA-based setup is designed in a similar manner as the feedback controller for the PEA-based setup. The frequency response of the controller is shown in figure 8.10a and the controller parameters are listed in table 8.6.

Once more, the feedback controller is basically a tame integrator with a second order high-pass filter. Some additional poles and zeros are added near $800 \mathrm{~Hz}$ to improve the stability margin in this frequency range.

The resulting minimum phase margin is $54^{\circ}$ at $108 \mathrm{~Hz}$ and the smallest gain margin has a value of $9.4(19 \mathrm{~dB}$, at $373 \mathrm{~Hz})$. The peak magnitude of the complementary sensitivity function is 1.17 at $97 \mathrm{~Hz}$. Therefore, multiplicative (relative) model errors of at least $85 \%$ are allowed at all frequencies.

The open loop and closed loop poles for the VCA-based setup, in the frequency range from 1 to $100 \mathrm{~Hz}$, are presented in table 8.6b. Note that the damping ratio of the suspension mode in open loop is six times larger compared to the PEA-based setup (see table 8.1). This is the result of the back-emf damping that is introduced by operating the voice coil actuator in voltage mode. 

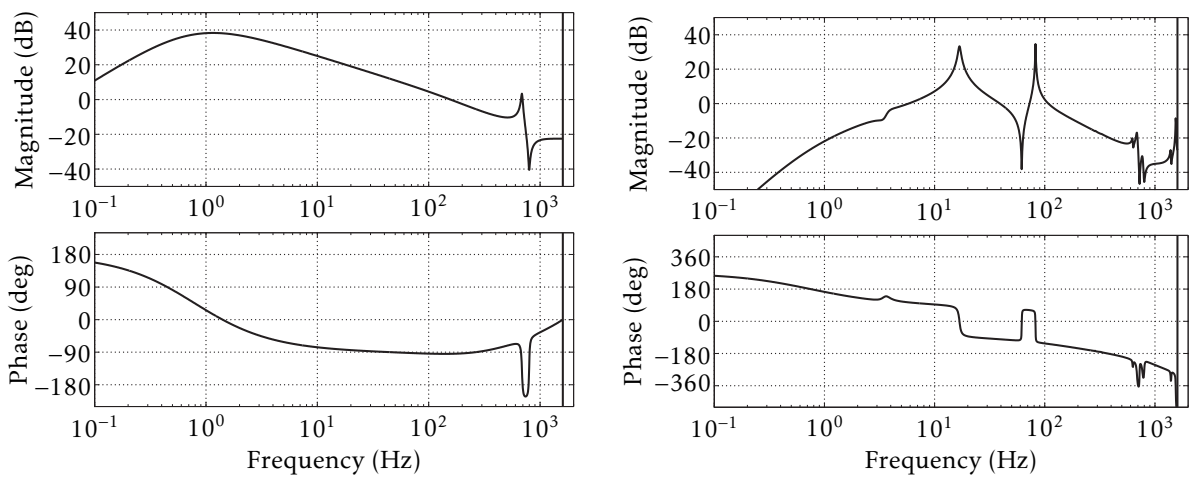

(a) Feedback controller

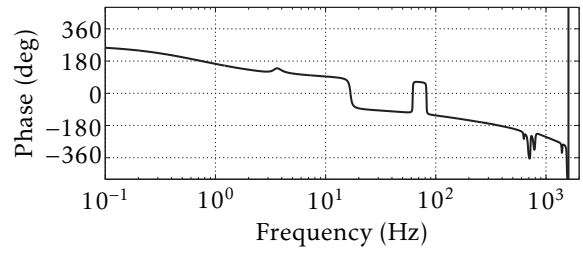

(b) Loop gain

Figure 8.10: Frequency response of the feedback controller (fig. a) and the resulting loop gain (fig. b) for the VCA-based setup.

The suspension mode has shifted to $79 \mathrm{~Hz}$ and its damping has improved to $37 \%$ (100-fold improvement). Moreover, the remaining poles in this frequency range (except the floor resonance) have damping ratios better than $40 \%$. It is therefore concluded that the objectives related to the damping of the suspension and structural modes are met.

Table 8.6: Feedback control of the VCA-based setup

(a) Controller parameters

\begin{tabular}{|c|c|c|}
\hline Gain & \multicolumn{2}{|c|}{0.186} \\
\hline Poles & $f(\mathrm{~Hz})$ & $\zeta(\%)$ \\
\hline & 0.61 & $($ real $)$ \\
\hline & 0.68 & $($ real $)$ \\
\hline & 1.2 & $($ real $)$ \\
\hline & 196 & $($ real $)$ \\
\hline & 688 & 1.5 \\
\hline Zeros & $f(\mathrm{~Hz})$ & $\zeta(\%)$ \\
\hline & 0 & $($ real, $2 \times)$ \\
\hline & 388 & $($ real $)$ \\
\hline & 798 & 1.1 \\
\hline & 1139 & $($ real $)$ \\
\hline
\end{tabular}

(b) Open and closed loop poles $(1-100 \mathrm{~Hz})$

\begin{tabular}{|c|c|c|}
\hline Open loop & $f(\mathrm{~Hz})$ & $\zeta(\%)$ \\
\hline & 3.7 & 11 \\
\hline & 17 & 1.6 \\
\hline & 82 & 0.31 \\
\hline Closed loop & $f(\mathrm{~Hz})$ & $\zeta(\%)$ \\
\hline & 1.8 & $($ real $)$ \\
\hline & 3.7 & 8.2 \\
\hline & 4.8 & $($ real $)$ \\
\hline & 79 & 37 \\
\hline & 89 & 58 \\
\hline
\end{tabular}




\section{Results}

The closed loop performance is illustrated in the frequency domain in figure 8.11. The open loop and closed loop power spectral densities of the acceleration $\ddot{x}_{1}$ of the machine bode $M_{1}$ are compared in figure $8.11 \mathrm{c}$. A similar comparison for the acceleration $\ddot{x}_{2}$ is shown in figure $8.11 \mathrm{~d}$. The effect of the increased damping of the suspension and structural modes is obvious.

In figures $8.11 \mathrm{~d}$ and $8.11 \mathrm{~b}$, the corresponding 1/3-octave RMS acceleration levels are presented. Moreover, the reference acceleration level is drawn for comparison. From these figures, it is clear that the feedback performance level is still worse than the reference performance level.

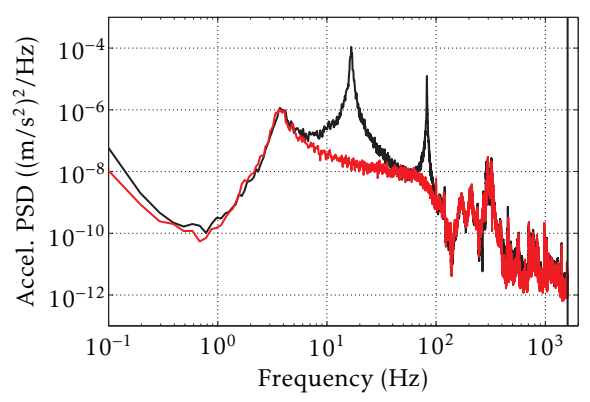

(a) Acceleration PSD of $M_{2}$

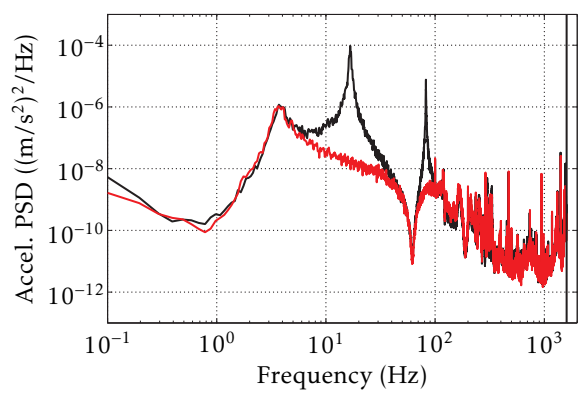

(c) Acceleration PSD of $M_{1}$

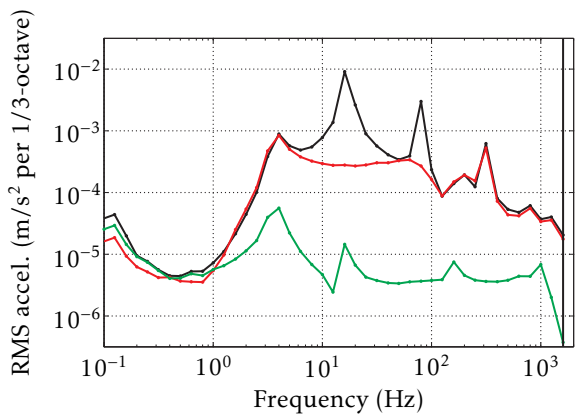

(b) RMS acceleration of $M_{2}$ (per 1/3-octave)

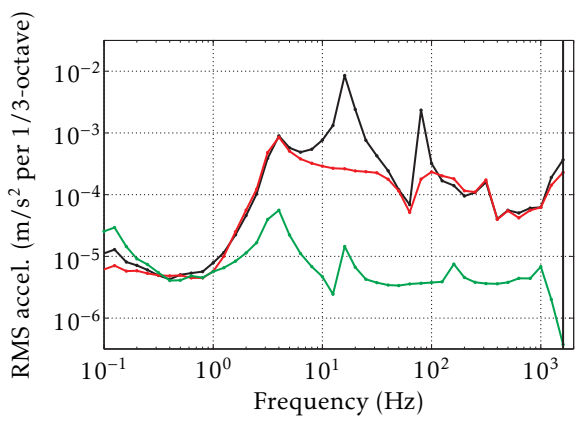

(d) RMS acceleration of $M_{1}$ (per 1/3-octave)

Figure 8.11: Comparison of open loop and closed loop machine acceleration levels of the VCA-based setup. Figures (a) and (b): machine mass $2\left(M_{2}\right)$, figures $(c)$ and $(d)$ : machine mass $1\left(M_{1}\right)$

- : open loop acceleration level

- : closed loop acceleration level

— : reference machine acceleration level (figs. $b, d$ ) 
Table 8.7: Performance measures for the VCA-based setup: Open loop (OL) and feedback controlled (FB)

\begin{tabular}{|c|c|c|c|}
\hline Measure & OL & FB & Unit \\
\hline reference (RMS) & 0.092 & 0.092 & $\mathrm{~mm} / \mathrm{s}^{2}$ \\
\hline$\ddot{x}_{0}($ RMS) & 17 & 16 & $\mathrm{~mm} / \mathrm{s}^{2}$ \\
\hline$\ddot{x}_{1}($ RMS $)$ & 9.5 & 1.5 & $\mathrm{~mm} / \mathrm{s}^{2}$ \\
\hline$\ddot{x}_{2}($ RMS) & 10 & 1.7 & $\mathrm{~mm} / \mathrm{s}^{2}$ \\
\hline$u_{a}($ RMS $)$ & - & $\begin{array}{c}0.16 \\
(0.096)\end{array}$ & $\begin{array}{c}\mathrm{V} \\
(\mathrm{N})\end{array}$ \\
\hline$u_{a}$ (pk-pk) & - & $\begin{array}{c}1.1 \\
(0.68)\end{array}$ & $\begin{array}{c}\mathrm{V} \\
(\mathrm{N})\end{array}$ \\
\hline$T_{\text {turn }}$ & 20.4 & 20.9 & $\mu \mathrm{s}$ \\
\hline
\end{tabular}

${ }^{a}$ All RMS values are total RMS $(0-1600 \mathrm{~Hz})$

This can also be concluded from the summary of the performance measures in table 8.7. The feedback controller has reduced the total RMS acceleration levels by a factor of six $(-16 \mathrm{~dB})$. However, the acceleration levels are still worse than the reference level, by a factor of $17(25 \mathrm{~dB})$.

Note that the open loop acceleration levels are approximately four times smaller than the open loop levels measured on the PEA-based setup (see e.g. table 8.5). This is partly caused by the increased damping of the suspension mode, but also by the fact that the suspension mode is now at $17 \mathrm{~Hz}$ instead of $34 \mathrm{~Hz}$. As a result, the contribution of the structural mode to the overall acceleration is reduced by roughly a factor four.

The required RMS control signal for the feedback controller is $0.16 \mathrm{~V}$, or equivalently $0.096 \mathrm{~N}^{1}$. The corresponding peak-peak signal is $1.1 \mathrm{~V}(0.68 \mathrm{~N})$. This value is approximately five times larger than the value predicted in section 6.2.1, as expected due to the larger excitation level.

Lastly, the required turnaround time is $20.9 \mu$ s, which amounts to only $6.7 \%$ of the sample interval and is only slightly more than the open loop turnaround time. Clearly, data acquisition, data logging and other housekeeping tasks are much more demanding than computing the feedback control signal.

\subsubsection{Causal Wiener filter}

Attempts to implement the Causal Wiener filter on the VCA-based setup have not been successful, for similar reasons as explained for the PEA-based setup, see section 8.2.3.

1 The required force is an estimate based on the nominal system gains, see also section 6.2.3. 
Therefore, the regularized Causal Wiener filter is again presented only to predict the achievable vibration isolation performance of the adaptive feedforward controller. The frequency response of the regularization filter $S_{\text {reg }}(z)$ and the closed loop secondary path $S_{\mathrm{cl}}(z)$ are shown in figure 8.12a. The standard Causal Wiener filter and the regularized Causal Wiener filter are compared in figure $8.12 \mathrm{~b}$, from which the effect of the regularization filter is obvious.

The predicted vibration isolation performance is computed from the identified model's frequency response to excitation by the shaker $\left(u_{s h}\right)$, using the closed loop transfer paths. In figure 8.13a, the open loop response, the closed loop response and the feedforward corrected response are shown for the acceleration $\ddot{x}_{2}$ of the second machine mass $M_{2}$. Similar frequency responses are shown in figure $8.13 \mathrm{~b}$ for the acceleration $\ddot{x}_{1}$ of the first machine mass $M_{1}$.

From figure 8.13a, it is concluded that the feedforward compensation can significantly improve the feedback performance up to approximately $150 \mathrm{~Hz}$, reaching $-25 \mathrm{~dB}$ at e.g. $10 \mathrm{~Hz}$. At frequencies above $150 \mathrm{~Hz}$, the second mass $M_{2}$ is effectively decoupled from the first machine mass $M_{1}$ and the feedforward compensation no longer has any effect (just like the feedback control). Because the feedforward controller is designed to minimize the acceleration $\ddot{x}_{1}$ of the first machine mass $M_{1}$ (the available error signal), this decoupling is not compensated for. The important conclusion is that the vibration isolation performance is only improved over the frequency range in which the error signal (in this case $\ddot{x}_{1}$ ) and the actual performance signal (in this case $\ddot{x}_{2}$ ) have a similar response to the disturbance sources.

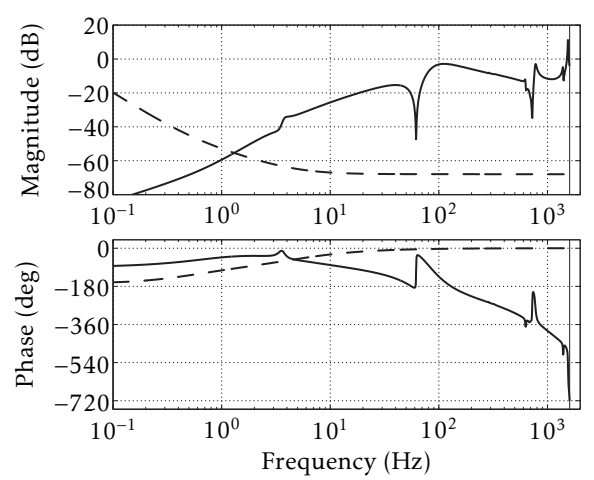

(a) Closed loop secondary path (-) and regularization filter (- -)
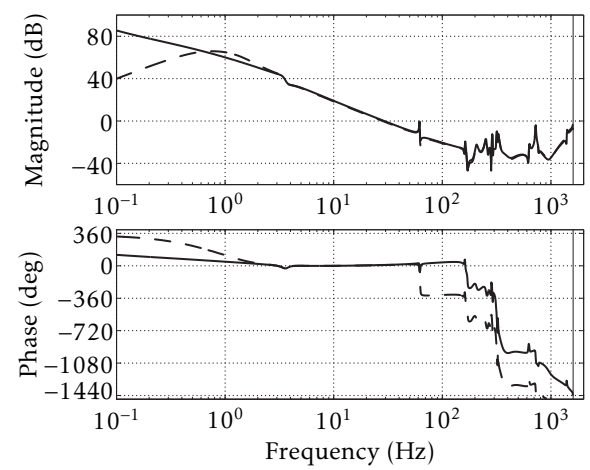

(b) Causal Wiener filter (-) and regularized Causal Wiener filter (- -)

Figure 8.12: Frequency responses of (a): secondary path (-) and regularization filter (- - , and (b): Causal Wiener filter (-) and regularized Causal Wiener filter (- -), for the VCA-based setup 


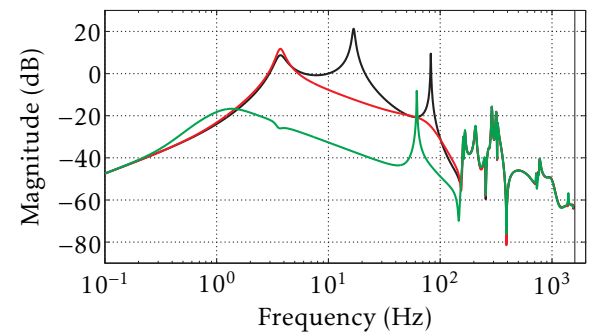

(a) $\ddot{X}_{2}(z) / U_{s h}(z)$

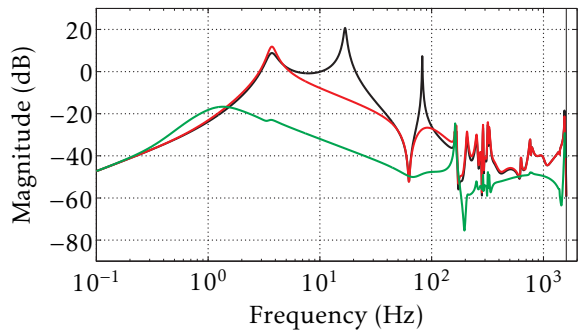

(b) $\ddot{X}_{1}(z) / U_{s h}(z)$

Figure 8.13: Frequency responses of (a): acceleration $\ddot{x}_{2}$ of machine mass $M_{2}$, and (b): acceleration $\ddot{x}_{1}$ of machine mass $M_{1}$, due to shaker excitation $u_{s h}$, for the VCA-based setup.

— : : open loop

— : feedback + regularized Causal Wiener filter

Just like for the PEA-based setup, the frequency response of $\ddot{x}_{2}$ is increased at the anti-resonance frequency of the secondary path $(62 \mathrm{~Hz})$. As shown in section 8.2 .4 , this effect can be counteracted by adding a notch filter to the noise shaping filter used in the adaptive feedforward controller.

Because the feedforward compensation is designed to minimize the error signal $\ddot{x}_{1}$, the error signal level is reduced from approximately $1 \mathrm{~Hz}$ to the Nyquist frequency at $1600 \mathrm{~Hz}$, see figure $8.13 \mathrm{~b}$. However, it has been observed in the experiments that have been performed on the PEA-based setup that the highfrequency performance is most likely not as good.

Moreover, because the tertiary path $T(z)$ still has an unstable zero pair at 160 $\mathrm{Hz}$ (see also figure 7.14a), it is not possible to achieve any reduction at this frequency.

\subsubsection{Adaptive feedforward using FIR filters}

In this section, the experimental results that have been obtained using an adaptive feedforward controller with an FIR parametrization are presented. The feedforward controller is implemented in exactly the same way as in the previous experiments on the PEA-based setup. A full block diagram of the implemented adaptive feedforward controller is presented in appendix F.1.

Table 8.8: FIR feedforward controller parameters for the VCA-based setup (see also appendix F.1)

\begin{tabular}{|c|c|c|c|c|c|}
\hline Parameter & $L$ & $\bar{\mu}$ & $\epsilon$ & $\gamma$ & $\rho$ \\
\hline Value & 2000 & 1 & 0.1 & $1 \cdot 10^{-5}$ & $1.75 \cdot 10^{-4}$ \\
\hline
\end{tabular}




\section{Parameter settings}

The controller parameters for the experiments are listed in table 8.8. Here, $L$ is the number of FIR parameters, $\bar{\mu}$ is the normalized step size (scale factor for the time-varying step size $\mu(k)), \epsilon$ is the regularization constant in the timevarying step size, $\gamma$ is the leakage factor and $\rho$ is the regularization constant that is applied to the closed loop secondary path (see also appendix F.1, equation (F.4)).

In this section, only the results for a typical experiment with $L=2000$ controller weights are presented. Many more experiments have been performed, with a varying number of controller weights. The results for these experiments are outlined in section 8.3.5, figure 8.20.

\section{Residual noise shaping}

The noise shaping filter $N(z)$ is designed to reduce the low-frequency content of the actuator signal. Moreover, it includes a notch filter at the anti-resonance frequency of the secondary path (at $62 \mathrm{~Hz}$ ). The frequency responses of the noise shaping filter $N(z)$ and its complement $M(z)$ are shown in figure 8.14. The noise shaping filter $N(z)$ now has dominant poles at approximately 0.5 $\mathrm{Hz}$ and $+40 \mathrm{~dB} /$ decade roll-on. The peak gain of $N(z)$ and its complement are both only 1.44 , so the amplification is limited. The complementary filter initially achieves $-40 \mathrm{~dB} /$ decade roll-off, but this behaviour is cut short by the presence of the notch filter in $N(z)$.

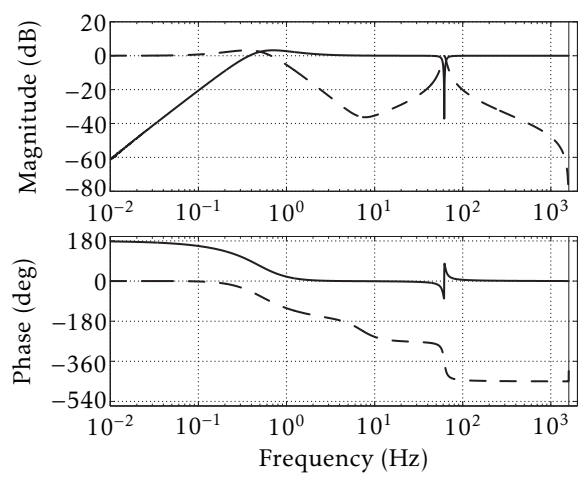

Figure 8.14: Frequency response of the noise shaping filter $N(z)(-)$ and its complement $M(z)=1-N(z)(--)$, for the VCA-based setup. 


\section{Results: frequency domain}

A good insight into the vibration isolation performance is obtained by analysing the power spectral density and the 1/3-octave RMS spectra of the measured acceleration signals. In figure 8.15 , the results obtained with the FIR feedforward control (combined with feedback control) are compared to the case in which only feedback control is applied (see also section 8.3.2).

Firstly, the vibration isolation performance is discussed in terms of the acceleration level of the second machine mass $M_{2}$. By comparing figure $8.15 \mathrm{a}$ to figure $8.13 \mathrm{a}$, it is concluded that the performance of the adaptive FIR feedforward controller is roughly as expected.

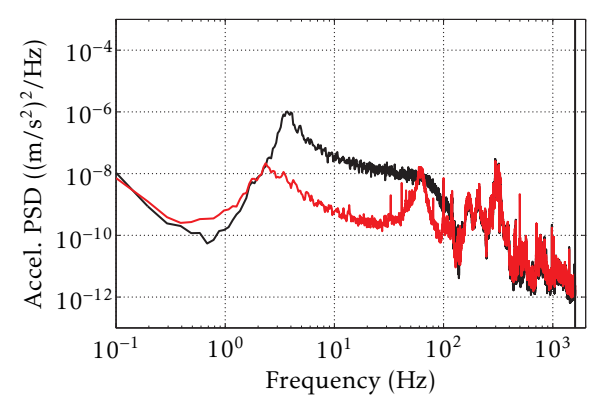

(a) Acceleration PSD of $M_{2}$

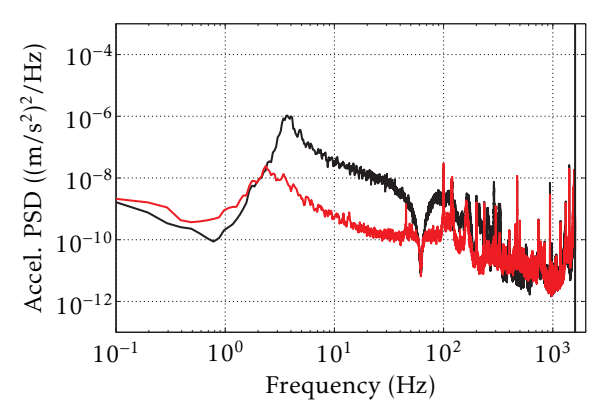

(c) Acceleration PSD of $M_{1}$

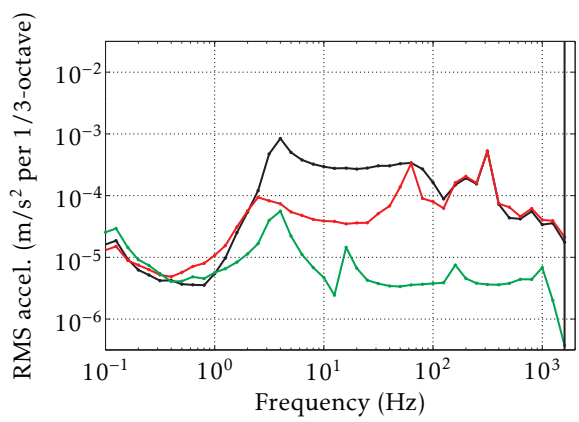

(b) RMS acceleration of $M_{2}$ (per 1/3-octave)

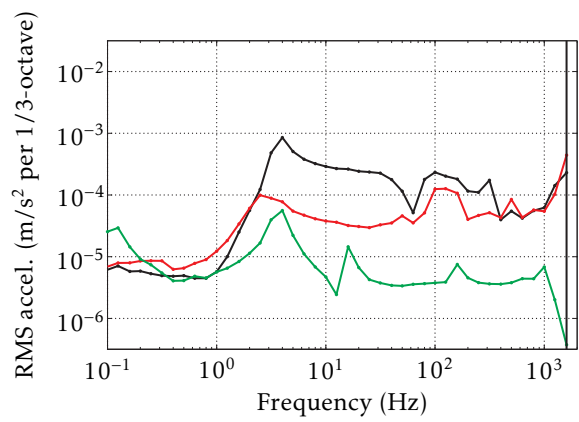

(d) RMS acceleration of $M_{1}$ (per 1/3-octave)

Figure 8.15: Comparison of machine acceleration levels for the VCA-based setup, for the (FIR) feedforward controlled and feedback controlled situation. See also table 8.8 and figure 8.14 for feedforward parameters

Fig. (a) and (b): machine mass $2\left(M_{2}\right)$, fig. (c) and $(d)$ : machine mass $1\left(M_{1}\right)$

- : Feedback controlled acceleration level

- : FIR feedforward compensated acceleration level

- : reference machine acceleration level (figs. $b, d$ ) 
In the frequency range from 2 to $100 \mathrm{~Hz}$, the acceleration level of the second machine mass $M_{2}$ is indeed reduced, although slightly less than predicted $(-20 \mathrm{~dB}$ instead of $-25 \mathrm{~dB}$ at $10 \mathrm{~Hz})$. Above $100 \mathrm{~Hz}$, no reduction is achieved, as expected due to the decoupling of the second machine mass.

Near $1 \mathrm{~Hz}$, some amplification of the acceleration level is observed, which is as expected due to the presence of the noise shaping filter $N(z)$. A similar amplification is predicted for the regularized Causal Wiener filter, meaning that the noise shaping filter has a similar effect as the regularization of the secondary path.

Moreover, the effect of the notch filter in $N(z)$ near the anti-resonance frequency of the secondary path at $62 \mathrm{~Hz}$ is clear: excessive peaking of the PSD is prevented (as predicted by the regularized Causal Wiener filter, see figure 8.13a).

From the 1/3-octave RMS spectrum in figure $8.15 \mathrm{~b}$ it is observed that the dominant contributions to the acceleration level of $M_{2}$ are in the frequency range from 160 to $300 \mathrm{~Hz}$ and at $60 \mathrm{~Hz}$. Moreover, the levels of these contributions are equal to the feedback controlled case. This is not surprising because of the structure of the control setup, as explained below:

- The secondary path has an anti-resonance frequency at $62 \mathrm{~Hz}$. Therefore, the adaptation algorithm is "blind" to any control action at this frequency. There is no guarantee that this "blind" control action will improve the vibration isolation performance and therefore it is best to do nothing, i.e. implement a notch filter.

- The adaptive algorithm uses the acceleration $\ddot{x}_{1}$ of $M_{1}$ as the error signal. As a result, it does not compensate for the decoupling of the secondary machine mass $M_{2}$. Obviously, using the acceleration $\ddot{x}_{2}$ as the error signal would eliminate this problem.

This is not surprising, as the adaptive algorithm achieves the best overall performance when the actual performance signal is used for the adaptation. In practice, this may be troublesome because the actual performance signal may not be measurable. In some cases, it might be possible to use an estimation of the performance signal instead, but this is a topic for further research.

Finally, from figure $8.15 \mathrm{~b}$ it is concluded that the target performance level is still worse than the achieved acceleration level, especially at frequencies above $10 \mathrm{~Hz}$, where the difference varies by factors of 10 to 100 . 
The performance in terms of the acceleration $\ddot{x}_{1}$ of the first machine mass $M_{1}$ is very similar, see figures $8.15 \mathrm{c}$ and $8.15 \mathrm{~d}$. In this case, the acceleration level is reduced over a larger frequency range, up to approximately $300 \mathrm{~Hz}$. However, the achieved performance is not as good as predicted by the regularized Causal Wiener filter (see figure 8.13b). The (suspected) causes for the reduced performance are listed below:

- The noise shaping filter with notch slightly reduces the performance near the notch frequency (see figure 8.14).

- An analysis of the noise contributions of the sensors and VCA power amplifier has shown that, above $400 \mathrm{~Hz}$, the acceleration signal $\ddot{x}_{1}$ is dominated by the effects of the VCA power amplifier noise (see section 6.2.3 for an overview of the analysis).

- It is suspected that several of the modes in this frequency range are actually tilting modes that are observed by the accelerometer on the first machine mass $M_{1}$, but are nearly uncontrollable by the VCA (note that these modes do not appear in the secondary path, see figure $7.14 \mathrm{~d}$ ). This structural property of the underlying system is not considered by the Wiener filter problem.

\section{Results: time traces}

Time traces of a typical experiment with the settings of table 8.8 are shown in figure 8.16. In figure 8.16a, the acceleration $\ddot{x}_{1}$ of the first machine mass $M_{1}$ is shown. The initial response is the open loop system response. Then, after approximately 20 seconds, the feedback controller is enabled, leading to a nearly immediate and significant reduction of the acceleration level. At approximately 41 seconds, the adaptive algorithm for the feedforward controller is switched on. After convergence of the error signal, which takes less than ten seconds, the acceleration level is reduced even further.

A similar behaviour is observed from figure $8.16 \mathrm{~b}$ for the acceleration $\ddot{x}_{2}$ of the second machine mass $M_{2}$, although the residual acceleration level is clearly larger compared to the acceleration $\ddot{x}_{1}$.

Time traces of five selected controller weights are shown in figure 8.17a. Although the error signal converges to its final value within ten seconds, it is clear that convergence of some of the controller weights takes much longer, e.g. approximately 60 seconds for the topmost time trace. Apparently, these controller weights have only a minor influence on the total error signal. 


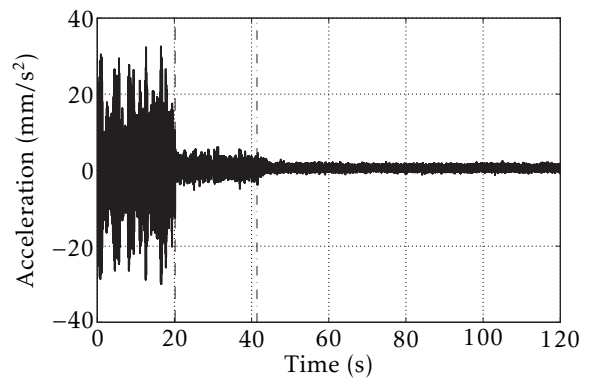

(a) Time trace of $\ddot{x}_{1}$

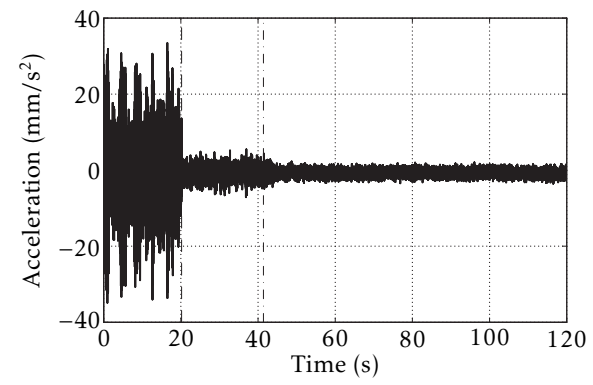

(b) Time trace of $\ddot{x}_{2}$

Figure 8.16: Time traces of (a) the machine mass $M_{1}$ acceleration, and (b) the machine mass $M_{2}$ acceleration. The feedback controller is switched on after 20 seconds and the adaptive feedforward controller is switched on after 41 seconds. Only every tenth time sample is plotted.

In figure $8.17 \mathrm{~b}$, the time trace of the control signal $u_{a}$ is shown. Clearly, the feedforward controller requires significantly larger control effort than the feedback controller. More importantly, a large transient response is visible starting at 41 seconds, right after the adaptive algorithm is enabled. The resulting maximum and minimum control signals are much larger than the steady state peak value, roughly by a factor of two. In this particular case, the transient behaviour is not a problem as the peaks are well within the DA-range of $\pm 5 \mathrm{~V}$. However, when such transient behaviour is undesired, it can be circumvented by choosing an initially smaller value for the step size parameter $\bar{\mu}$ and gradually increasing its value.

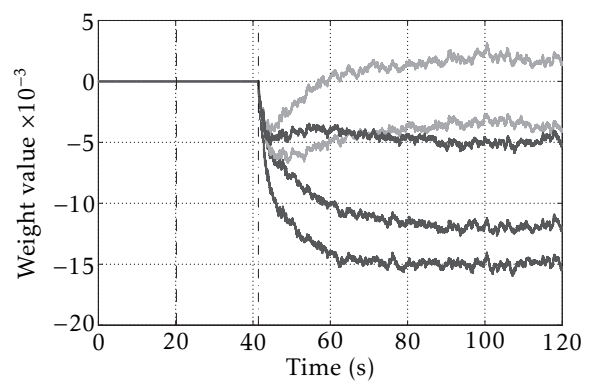

(a) Time trace of selected controller weights

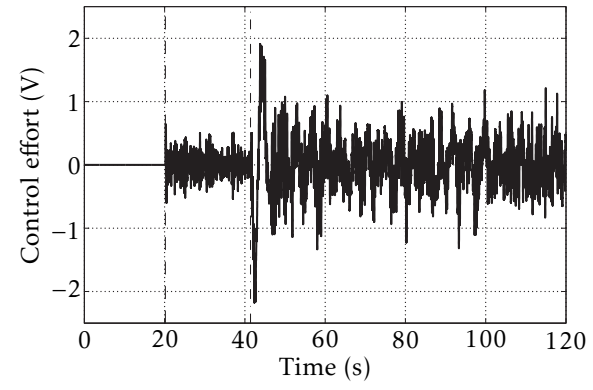

(b) Time trace of total control effort

Figure 8.17: Time traces of (a) five (out of 2000) controller weights, and (b) the total control effort $u_{a}$. The feedback controller is turned on after 20 seconds and the adaptive feedforward controller is turned on after 41 seconds. Only every tenth time sample is plotted. 


\section{Results: summary}

The results for the adaptive feedforward controller with FIR structure $(L=$ 2000) are summarized in table 8.9 , together with the previously obtained results for the open loop and feedback controlled experiments.

The acceleration level $\ddot{x}_{2}$ is reduced by another $55 \%(-7 \mathrm{~dB})$ compared to the feedback performance. For the acceleration signal $\ddot{x}_{1}$, the reduction amounts $62 \%(-8 \mathrm{~dB})$. However, both residual acceleration levels are still an order of magnitude above the reference level.

The control signal that is generated by the feedforward controller is twice as large compared to the feedback controlled case. ${ }^{2}$ Lastly, the required computational time has increased tenfold, due to the large amount of controller weights $(L=2000)$.

Table 8.9: Performance measures for the VCA-based setup: Open loop (OL), feedback controlled (FB) and FIR feedforward compensated (FF FIR, $L=2000$ )

\begin{tabular}{|c|c|c|c|c|}
\hline Measure & OL & FB & $\begin{array}{c}\text { FF FIR } \\
(L=2000)\end{array}$ & Unit \\
\hline reference (RMS) & 0.092 & 0.092 & 0.092 & $\mathrm{~mm} / \mathrm{s}^{2}$ \\
\hline$\ddot{x}_{0}(\mathrm{RMS})$ & 17 & 16 & 16 & $\mathrm{~mm} / \mathrm{s}^{2}$ \\
\hline$\ddot{x}_{1}(\mathrm{RMS})$ & 9.5 & 1.5 & 0.57 & $\mathrm{~mm} / \mathrm{s}^{2}$ \\
\hline$\ddot{x}_{2}($ RMS $)$ & 10 & 1.7 & 0.77 & $\mathrm{~mm} / \mathrm{s}^{2}$ \\
\hline$u_{a}($ RMS $)$ & - & 0.16 & 0.37 & $\mathrm{~V}$ \\
\hline$u_{a}($ pk-pk) & - & $\begin{array}{c}1.1 \\
(0.68)\end{array}$ & $\begin{array}{c}2.4 \\
(1.4)\end{array}$ & $\begin{array}{c}\mathrm{V} \\
(\mathrm{N})\end{array}$ \\
\hline$T_{\text {turn }}$ & 20.4 & 20.9 & 200 & $\mu \mathrm{s}$ \\
\hline
\end{tabular}

a All RMS values are total RMS $(0-1600 \mathrm{~Hz})$

\subsubsection{Adaptive feedforward using IIR filters}

In this section, the experimental results that have been obtained using an adaptive feedforward controller with an IIR parametrization are presented. As discussed in section 5.3.5, the same FxLMS algorithm and its extensions can be applied to the IIR parametrization. To allow a fair comparison between the FIR and IIR parametrizations, the same adaptation algorithm has been used, see also appendix F.1 for a block diagram of the controller implementation.

2 In steady state, see figure $8.17 \mathrm{~b}$ for an illustration of transient effects 
Moreover, exactly the same settings have been used for the common parameters of the adaptive algorithms, see table 8.8 for these parameter settings and figure 8.14 for the implemented noise shaping filter. Moreover, the same feedback controller and the same excitation signal have been used in these experiments.

\section{Parameter settings: IIR1}

The choice for the poles has been based on the regularized Causal Wiener filter. By repetitively using model reduction routines (with increasing tolerance), a $21^{\text {st }}$ order filter is obtained that is still in close agreement with the regularized Causal Wiener filter. The remaining nine complex-conjugate pole pairs and three first order poles of this reduced filter are used for the second order sections (SOS) and the first order sections (FOS), see table 8.10.

The results of the adaptive feedforward control experiment with this choice of pole locations are denoted by IIR1, in order to distinguish them from results that are obtained with another choice for the pole locations. The latter results are presented in a later section.

Table 8.10: Pole locations for the IIR1 filter

\begin{tabular}{|c|c|c|}
\hline SOS & Freq. $\mathbf{( H z )}$ & $\zeta(\mathbf{\%})$ \\
\hline $0.7259 \pm 0.5783 j$ & 344.7 & 11.0 \\
\hline $0.8078 \pm 0.5802 j$ & 317.2 & 0.872 \\
\hline $0.8416 \pm 0.5327 j$ & 287.4 & 0.712 \\
\hline $0.8485 \pm 0.5028 j$ & 272.6 & 2.58 \\
\hline $0.8726 \pm 0.4715 j$ & 252.3 & 1.67 \\
\hline $0.9101 \pm 0.3899 j$ & 206.2 & 2.46 \\
\hline $0.9476 \pm 0.3126 j$ & 162.3 & 0.686 \\
\hline $0.9607 \pm 0.1422 j$ & 76.30 & 19.5 \\
\hline $0.99934 \pm 0.007195 j$ & 3.681 & 8.77 \\
\hline
\end{tabular}

\begin{tabular}{|c|c|}
\hline FOS & Freq. $\mathbf{( H z )}$ \\
\hline 0.6185 & 244.7 \\
\hline 0.9074 & 49.50 \\
\hline 0.9897 & 5.257 \\
\hline
\end{tabular}

\section{Results: IIR1}

Recall from section 5.3.5 that the IIR filter structure is proposed because the real-time implementation would then require less computation time. This is indeed the case, as the turnaround time is decreased from $200 \mu$ s for the FIR implementation (with 2000 weights) to only $27 \mu$ s for the IIR filter with the poles as listed in table 8.10 . The latter turnaround time is only a $30 \%$ increase compared to the feedback controller. 
Then, it remains to be seen that the IIR parametrization obtains the same or better performance than the FIR parametrization. In figure 8.18 , the results for the IIR1 experiment (red) are compared to the feedback response (black) and the FIR performance (blue). From the PSD plots (figures 8.18c and 8.18a) not much can be concluded, other than that the performance is similar. In this respect, the 1/3-octave RMS plots are more insightful. The most important difference between the FIR and IIR1 performance is that, for the latter, there is no longer any performance improvement (over the feedback controller) in the frequency range 150-300 Hz. Because the RMS levels are relatively high in this range, the overall RMS acceleration is significantly increased.

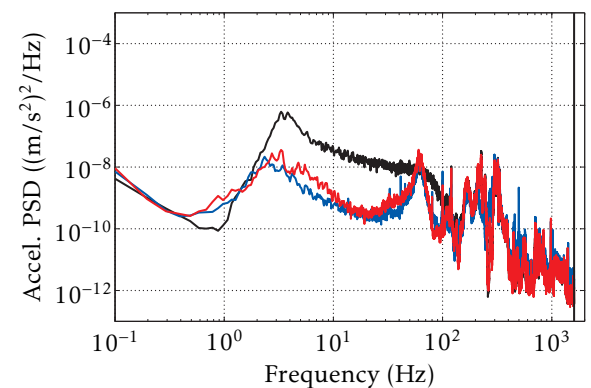

(a) Acceleration PSD of $M_{2}$

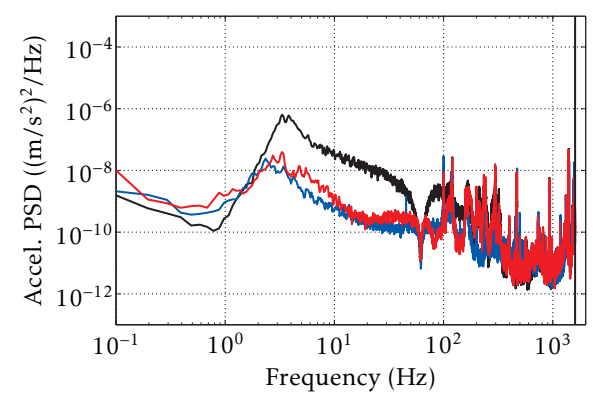

(c) Acceleration PSD of $M_{1}$

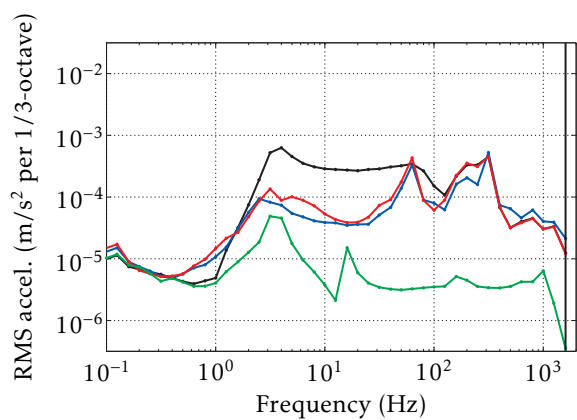

(b) RMS acceleration of $M_{2}$ (per 1/3-octave)

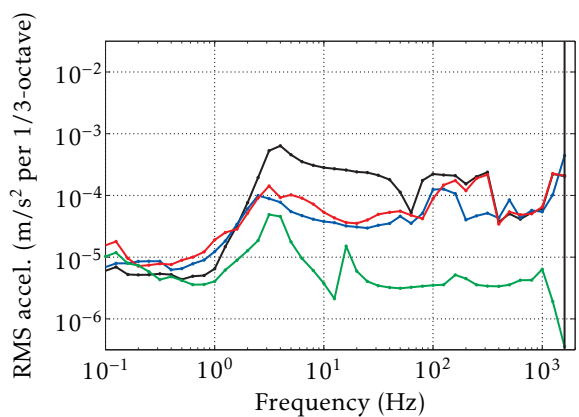

(d) RMS acceleration of $M_{1}$ (per 1/3-octave)

Figure 8.18: Comparison of machine acceleration levels for the VCA-based setup, for the IIR1 feedforward controlled, FIR feedforward controlled and feedback controlled situation. See also tables 8.8 and 8.10 for the feedforward parameters and pole locations.

Fig. (a) and (b): machine mass $2\left(M_{2}\right)$, fig. (c) and (d): machine mass $1\left(M_{1}\right)$

— : Feedback controlled acceleration level

— : FIR feedforward compensated acceleration level

— : IIR1 feedforward compensated acceleration level

- : reference machine acceleration level (figs. $b, d$ ) 
However, from figure $8.18 \mathrm{~d}$, it is observed that the RMS contribution at the Nyquist frequency $(1600 \mathrm{~Hz})$ is reduced (compared to the FIR plot). This reduction will balance the performance loss in the other frequency range for the acceleration of the first machine mass $M_{1}$.

The total RMS acceleration levels of the first and second machine mass are now 0.59 and $0.90 \mathrm{~mm} / \mathrm{s}^{2}$ respectively, compared to 0.57 and $0.77 \mathrm{~mm} / \mathrm{s}^{2}$ for the FIR feedforward controller $(L=2000)$. The performance loss for the first machine mass is indeed very moderate. However, the acceleration $\ddot{x}_{2}$ is increased by approximately 15\%, primarily due to the increased RMS level at 200 and $250 \mathrm{~Hz}$.

In the following sections, it is tested whether the performance of the feedforward controller with IIR parametrization can be improved by choosing other pole locations for the IIR filter.

\section{Parameter settings: IIR2}

For this second experiment, the pole locations of the IIR filter are chosen as the poles of the regularized Causal Wiener filter that occur at a frequency larger than $0.5 \mathrm{~Hz}$, i.e. no model reduction is performed. This set of poles includes 24 complex-conjugate pole pairs and 10 real poles, resulting in a $58^{\text {th }}$ order filter. The poles of the IIR1 filter are contained within this set. The full set of poles is listed in appendix F.2, table F.1. Most of the added poles are in the high-frequency range (above $100 \mathrm{~Hz}$ ), with two notable exceptions: real poles are added at 0.98 and $1.4 \mathrm{~Hz}$.

Moreover, the cut-off frequency of the noise shaping filter $N(z)$ has been shifted down from 0.5 to $0.25 \mathrm{~Hz}$. This will reduce the regularization effect on the control signal. This is justified because the peak-peak control signal in the IIR1 experiment is only $50 \%$ of the full DA-range.

\section{Results: IIR2}

In figure 8.19, the results obtained with the IIR2 feedforward filter are compared to the performances obtained with the FIR controller and the feedback controller. Clearly, the performance in the low frequency range $(2-20 \mathrm{~Hz})$ is improved. This improvement is caused by the addition of the two lowfrequency real poles. At higher frequencies, the response is similar to the IIR1 results, i.e. there is no improvement over the feedback performance. 


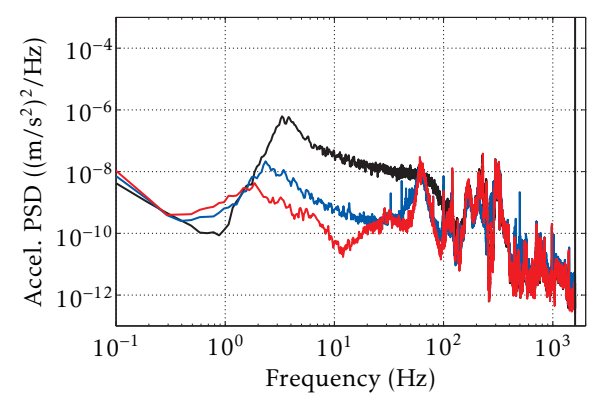

(a) Acceleration PSD of $M_{2}$

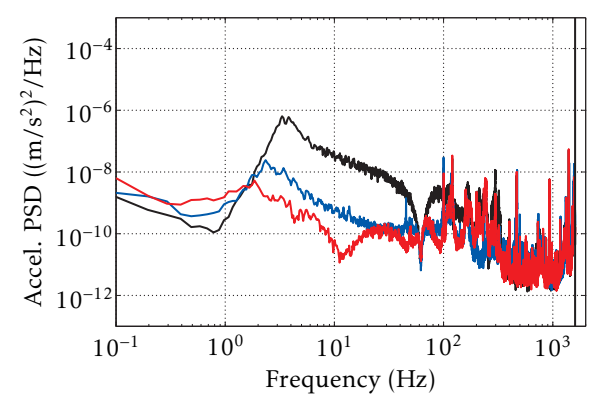

(c) Acceleration PSD of $M_{1}$

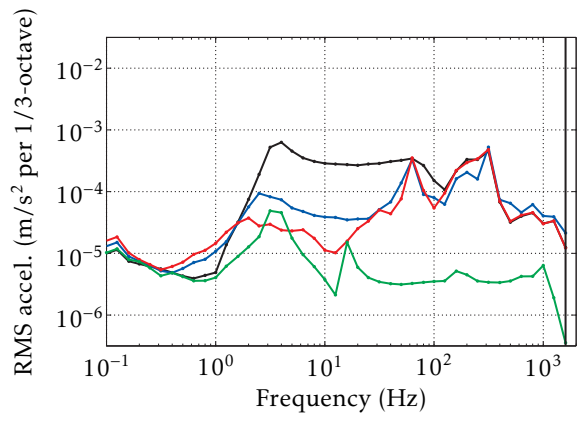

(b) RMS acceleration of $M_{2}$ (per 1/3-octave)

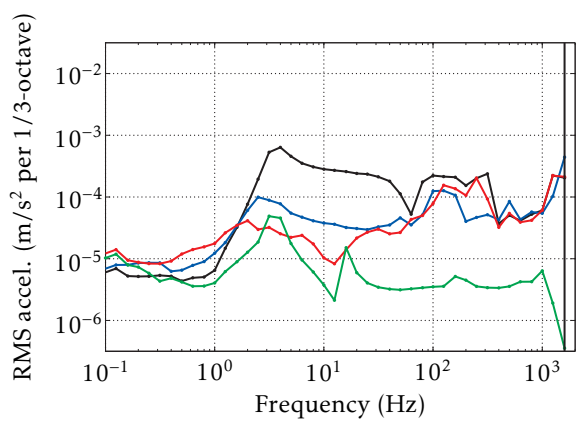

(d) RMS acceleration of $M_{1}$ (per 1/3-octave)

Figure 8.19: Comparison of machine acceleration levels for the VCA-based setup, for the IIR2 feedforward controlled, FIR feedforward controlled and feedback controlled situation. See also tables 8.8 and F.1 for the feedforward parameters and pole locations.

Fig. (a) and (b): machine mass $2\left(M_{2}\right)$, fig. (c) and (d): machine mass $1\left(M_{1}\right)$

- : Feedback controlled acceleration level

— : FIR feedforward compensated acceleration level

- : IIR2 feedforward compensated acceleration level

- : reference machine acceleration level (figs. $b, d$ )

The total RMS acceleration levels of the first and second machine mass are now 0.48 and $0.81 \mathrm{~mm} / \mathrm{s}^{2}$ respectively, compared to 0.57 and $0.77 \mathrm{~mm} / \mathrm{s}^{2}$ for the FIR feedforward controller $(L=2000)$. In this case, there is an additional $15 \%$ reduction of the acceleration level $\ddot{x}_{1}$ compared to the FIR result. The performance loss in terms of the acceleration of the second machine mass is only $5 \%$. Once more, this is primarily due to the increased RMS level at 200 and $250 \mathrm{~Hz}$.

Even though the IIR filter order is increased from 21 to 58, the total computational time is only increased by $4 \mu$ s to $31 \mu \mathrm{s}$, which is just under $10 \%$ of the sample interval. 


\section{Results: summary}

The results for the adaptive feedforward controller with IIR structure are summarized in table 8.11, together with the previously obtained results for the open loop, feedback controlled and FIR feedforward controlled experiments.

Moreover, the results of the various experiments are summarized graphically in figure 8.20. Here, the results of open loop (OL) and feedback control (FB) experiments are compared to various feedforward control experiments. Besides the FIR parametrized feedforward control experiment of section 8.3.4 $(L=2000)$, the results of additional experiments with different numbers of controller weights $L$ are included. The results from the IIR1 and IIR2 experiments are shown as well.

Figure 8.20a shows the total RMS accelerations of the first machine mass $M_{1}$ $\left(\ddot{x}_{1}\right)$ and the second machine mass $M_{2}\left(\ddot{x}_{2}\right)$. The feedback controller reduces the acceleration level by $84 \%(-16 \mathrm{~dB})$. Then, by increasing the number of controller weights for the FIR feedforward controller, the residual acceleration levels are reduced further, reaching a constant level of approximately 0.55 and $0.8 \mathrm{~mm} / \mathrm{s}^{2}$ for $\ddot{x}_{1}$ and $\ddot{x}_{2}$ respectively, for approximately 1000 controller weights or more. The reductions with respect to the open loop response are respectively $95 \%(-25 \mathrm{~dB})$ and $92 \%(-22 \mathrm{~dB})$. The performance of the feedforward controller using the IIR parametrization is similar. However, all these acceleration levels are still a factor of five to ten above the reference level.

Table 8.11: Performance measures for the VCA-based setup: Open loop (OL), feedback controlled (FB), FIR feedforward compensated (FF FIR, $L=2000$ ) and IIR feedforward compensated (IIR1, IIR2)

\begin{tabular}{|c|c|c|c|c|c|c|}
\hline Measure & OL & FB & $\begin{array}{c}\text { FF FIR } \\
(L=2000)\end{array}$ & FF IIR1 & FF IIR2 & Unit \\
\hline ref. (RMS $\left.{ }^{\mathrm{a}}\right)$ & 0.092 & 0.092 & 0.092 & 0.092 & 0.092 & $\mathrm{~mm} / \mathrm{s}^{2}$ \\
\hline$\ddot{x}_{0}($ RMS $)$ & 17 & 16 & 16 & 15 & 15 & $\mathrm{~mm} / \mathrm{s}^{2}$ \\
\hline$\ddot{x}_{1}($ RMS $)$ & 9.5 & 1.5 & 0.57 & 0.59 & 0.48 & $\mathrm{~mm} / \mathrm{s}^{2}$ \\
\hline$\ddot{x}_{2}$ (RMS) & 10 & 1.7 & 0.77 & 0.90 & 0.81 & $\mathrm{~mm} / \mathrm{s}^{2}$ \\
\hline$u_{a}($ RMS $)$ & - & 0.16 & 0.37 & 0.32 & 0.57 & $\mathrm{~V}$ \\
\hline$u_{a}$ (pk-pk) & - & $\begin{array}{c}1.1 \\
(0.68)\end{array}$ & $\begin{array}{c}(1.4) \\
(0.22)\end{array}$ & $\begin{array}{c}(0.19) \\
(1.4)\end{array}$ & $\begin{array}{c}(2.34) \\
(\mathrm{N})\end{array}$ \\
\hline$T_{\text {turn }}$ & 20.4 & 20.9 & 200 & 27 & 31 & $\mathrm{~V}$ \\
\hline
\end{tabular}

${ }^{a}$ All RMS values are total RMS $(0-1600 \mathrm{~Hz})$ 


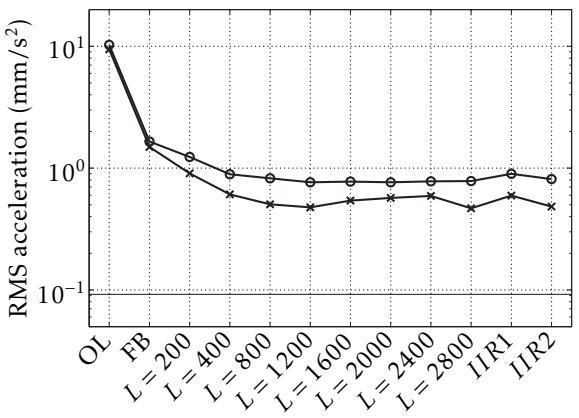

(a) RMS accelerations for various experiments $\left(\multimap: \ddot{x}_{1},-: \ddot{x}_{2},-:\right.$ reference $)$

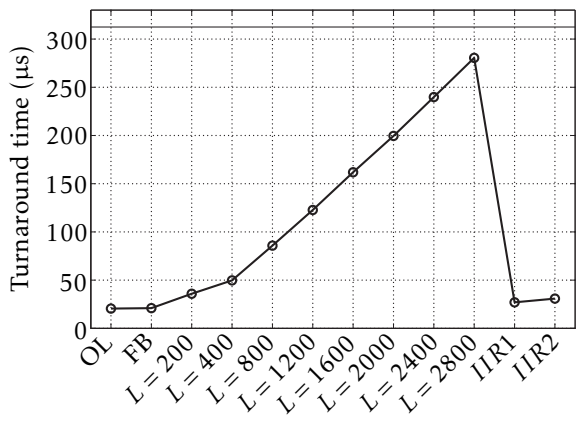

(b) Turnaround time $(-)$ for various experiments (-: sample interval)

Figure 8.20: Overview of the experimental results for various experiments performed on the VCA-based setup. The experiments with FIR feedforward controllers are indicated by their respective number of controller weights $L$.

In figure $8.20 \mathrm{~b}$, the turnaround time of the real-time implementation is drawn for the various experiments. As expected from section 5.3.6, the turnaround time for the FIR parametrization follows a linear trend (after removing the overhead time). With the chosen sample interval, a maximum of approximately 3000 controller weights can be implemented. Clearly, the IIR parametrization is more efficient than the FIR parametrization, while achieving a similar performance level.

\section{Results: Floor vibration transmissibility}

Because most industrial systems are advertised by their transmissibility, it is worthwhile to present the best performance that is achieved in this thesis in the same form. For this purpose, the estimated floor vibration transmissibility for the IIR2 experiment is shown in figure 8.21.

In figure $8.21 \mathrm{a}$, the transmissibility in terms of the machine acceleration $\ddot{x}_{1}$ is displayed. The feedforward control results are compared to the reference transmissibility $T_{\text {ref }}(s)$ as well as the open loop and feedback control results. Up to $10 \mathrm{~Hz}$, the performance achieved by the IIR 2 adaptive feedforward controller is roughly comparable to the reference transmissibility. This is quite remarkable as the open loop suspension frequency of this system lies at $17 \mathrm{~Hz}$. The amplification near $1 \mathrm{~Hz}$ is partly caused by measurement errors (the signal-to-noise ratio in this frequency range is rather poor) and partly by the regularization of the secondary path and the noise shaping filter. These measures are required to prevent the actuator signal from saturating. It might be possible to reduce the peak by a careful redesign of the regularization filter and the noise shaping filter. But more importantly, the signal-to-noise ratio should be reduced in this frequency range. 


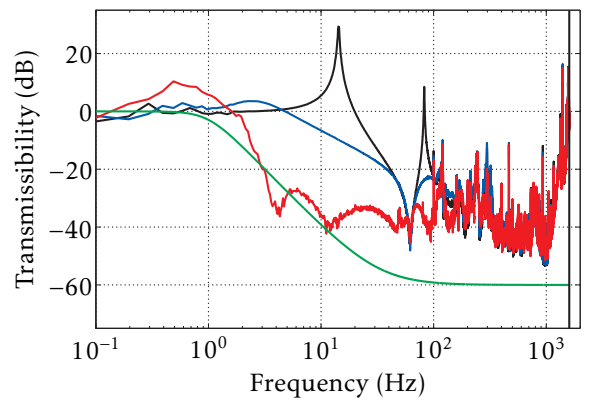

(a) Machine mass $M_{1}: \ddot{X}_{1}(f) / \ddot{X}_{0}(f)$

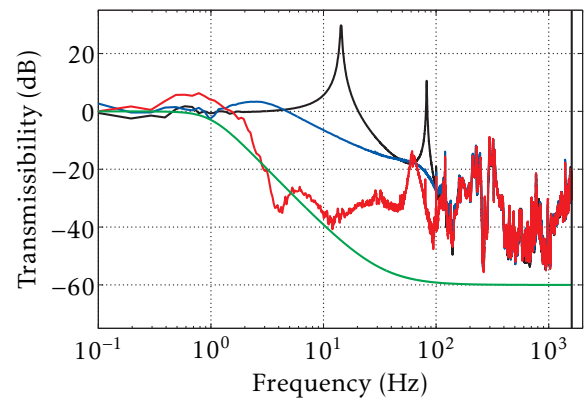

(b) Machine mass $M_{2}: \ddot{X}_{2}(f) / \ddot{X}_{0}(f)$

Figure 8.21: Floor vibration transmissibility for the IIR2 experiment.

— : open loop; — : feedback control; _ _ IIR2 feedforward;

— : reference transmissibility

At high frequencies, the measured transmissibility levels off and contains many spikes. As mentioned previously, the active control system is not capable of improving the performance in this frequency range due to the delays in the system. Therefore, the control bandwidth must be increased or the passive isolation performance at high frequencies must be improved.

The increase in the transmissibility above $1 \mathrm{kHz}$ is due to the noise injected by the VCA amplifier.

In figure $8.21 \mathrm{~b}$, the transmissibility functions in terms of the machine acceleration $\ddot{x}_{2}$ are displayed.

The overall performance of the IIR2 feedforward controller in terms of $\ddot{x}_{2}$ is similar to that of figure $8.21 \mathrm{~b}$. At low frequencies, the transmissibility is comparable to the reference transmissibility. Above $100 \mathrm{~Hz}$, there is no improvement compared to the open loop response. For the feedback controller, this is caused by the limited control bandwidth. The feedforward controller does not achieve any reduction of the transmissibility in this frequency range, because the error signal that is used for adaptation $\left(\ddot{x}_{1}\right)$ and the actual performance signal $\left(\ddot{x}_{2}\right)$ have a very different frequency response at these frequencies.

Moreover, the adverse effect of the anti-resonance frequency in the secondary path at $62 \mathrm{~Hz}$ on the transmissibility is clearly observed. The active control system contains a notch at this frequency. As a result, the performance can not be improved at this frequency.

\subsection{Recapitulation}

The DVF approach for the feedback controller works very well in improving the damping of the suspension and structural modes. The feedback controller is designed to achieve maximum damping in the structural mode. For both 
setups, the structural damping has been improved to $37 \%$. The suspension damping is at least $70 \%$. Depending on the open loop damping ratio, the acceleration levels are reduced by a factor 5-10 due to the increased damping.

The implemented feedforward controller uses preconditioning of the secondary path and swapping of the plant and controller to improve the convergence rate. Moreover, it uses a noise shaping filter to reduce the spectral content of the control signal at low frequencies and at the anti-resonance frequency of the secondary path at $62 \mathrm{~Hz}$. This regularization of the control signal is essential, as the control signal (actually the DA-converter) would otherwise saturate, leading to instability of the controller weight update.

The adaptive feedforward controller is able to further reduce the acceleration levels by a factor of 2-3. This improvement is almost completely obtained at relatively low frequencies (below $100 \mathrm{~Hz}$ ). Above this frequency, the error signal and the actual performance signal are no longer strongly correlated and any reduction of the measured error signal in this frequency range is not carried over to the actual performance signal.

In table 8.12, the results of the presented experiments are summarized once more. These results have been obtained using the same excitation for both setups, resulting in (open loop) total RMS floor acceleration levels of 20 and $17 \mathrm{~mm} / \mathrm{s}^{2}$ for the PEA-based and VCA-based setup respectively.

The best performance achieved on the PEA-based setup reduces the total RMS acceleration level $\ddot{x}_{2}$ to $1.7 \mathrm{~mm} / \mathrm{s}^{2}$, which corresponds to an internal deformation of roughly $11 \mathrm{~nm}$ (recall equation (7.3)). On the VCA-based setup, the best performance in terms of $\ddot{x}_{2}$ is $0.77 \mathrm{~mm} / \mathrm{s}^{2}$ (approx. $5 \mathrm{~nm}$ internal deformation).

Table 8.12: Summary of experimental results

\begin{tabular}{|c|c|c|c|c|c|c|c|}
\hline \multirow{2}{*}{ Experim. } & \multirow{2}{*}{$\begin{array}{c}\ddot{x}_{2}(\mathrm{RMS})^{\mathrm{a}} \\
\left(\mathrm{mm} / \mathrm{s}^{2}\right)\end{array}$} & \multirow{2}{*}{$\begin{array}{c}\ddot{x}_{1}(\mathrm{RMS}) \\
\left(\mathrm{mm} / \mathrm{s}^{2}\right)\end{array}$} & \multicolumn{2}{|c|}{$u_{a}(\mathrm{RMS})$} & \multicolumn{2}{|c|}{$u_{a}(\mathrm{pk}-\mathrm{pk})$} & \multirow{2}{*}{$\begin{array}{c}T_{\text {turn }} \\
(\mu \mathrm{s})\end{array}$} \\
\hline & & & $(\mathrm{V})$ & $(\mathrm{N})$ & $(\mathrm{V})$ & $(\mathrm{N})$ & \\
\hline PEA OL & 51 & 39 & - & - & - & - & 20.4 \\
\hline PEA FB & 4.9 & 3.6 & 0.41 & $(0.19)$ & 3.1 & $(1.5)$ & 20.6 \\
\hline PEA FIR1 & 2.2 & 1.1 & 2.0 & $(0.94)$ & 15 & $(7.0)$ & 293 \\
\hline PEA FIR2 & 1.7 & 0.96 & 1.9 & $(0.89)$ & 15.4 & $(7.2)$ & 252 \\
\hline VCA OL & 10 & 9.5 & - & - & - & - & 20.4 \\
\hline VCA FB & 1.7 & 1.5 & 0.16 & $(0.096)$ & 1.1 & $(0.68)$ & 20.9 \\
\hline VCA FIR & 0.77 & 0.57 & 0.37 & $(0.22)$ & 2.4 & $(1.4)$ & 200 \\
\hline VCA IIR1 & 0.90 & 0.59 & 0.32 & $(0.19)$ & 2.3 & $(1.4)$ & 27 \\
\hline VCA IIR2 & 0.81 & 0.48 & 0.57 & $(0.34)$ & 3.9 & $(2.3)$ & 31 \\
\hline
\end{tabular}

a All RMS values are total RMS $(0-1600 \mathrm{~Hz})$ 
These acceleration levels lie a factor of five to ten above the reference level, which is based on the (idealized) performance of a state-of-the-art soft mount system. However, it should be noted that the support stiffness of the setups are respectively 1200 and 300 times larger than the stiffness of the reference soft mount system (when supporting the same machine mass).

The following factors limiting the achievable performance have been discussed:

- The error signal and actual performance signal deviate at high frequencies (above $100 \mathrm{~Hz}$ )

- No improvement (near anti-resonance frequency) due to the undamped anti-resonance in the secondary path

- No improvement at the frequency of the unstable complex-conjugate zero pair in the tertiary path

- Low frequency sensor noise requires regularization of the control signal (to prevent actuator signal saturation)

- Amplifier noise (for the VCA, at high frequencies)

In view of the application of the adaptive feedforward controller algorithm in a setup with multiple channels, it is observed that using an IIR parametrization for the controller is a useful alternative to the more common FIR parametrization. The vibration isolation performance can be as good as or maybe better than the FIR parametrization, but the required computational time is reduced from $200 \mu$ s (or more) to approximately $30 \mu$ s. However, it has been shown that the performance depends critically on the choice of the pole locations of the IIR filter.

Several suggestions to improve the overall performance are listed below:

- The suspension stiffness should not be chosen higher than strictly necessary. Otherwise, the open loop transmissibility is increased and the required actuator signal is roughly proportional to the suspension stiffness.

- A redesign of the hard mount should be realized such that the sensors and the actuator are placed in-line. Then, the unstable zero pair in the tertiary path is eliminated, as it is caused by the off-axis placement of the accelerometer on the floor mass $M_{0}$.

- An error signal should be used that more closely resembles the desired performance. This may require additional sensors, such as force sensors.

- Improvement of the high-frequency passive vibration isolation. The active isolation has difficulties reducing the high-frequency vibrations (above $100 \mathrm{~Hz}$ ). It should be investigated whether a mechanical design can be realized that maintains sufficient (static) suspension stiffness, but achieves higher roll-off in the transmissibility function. 


\section{Chapter 9}

\section{Conclusions and recommendations}

In this chapter, the conclusions from the various theoretical and experimental results that have been presented in this thesis are summarized in section 9.1. Then, recommendations for further research are discussed in section 9.2.

\subsection{Conclusions}

The aim of this research project has been to develop a novel active hard mount vibration isolation concept. The hard mount support is intended to improve the robustness to direct disturbances by its relatively high support stiffness, but the floor vibration isolation leaves much to be desired. In this thesis, an active control approach is described that significantly improves the floor vibration isolation performance.

The active control system employs a combination of feedback and feedforward control. The feedback control is aimed at adding active suspension and structural damping. The feedforward control is used to compensate for measured floor vibrations.

In the following section, the results of the control experiments are summarized. Following this summary, the conclusions regarding each of the system components (feedback control, feedforward control, sensors, actuators and mechanical design) are presented in more detail in separate sections. 


\subsubsection{Experimental results (chapter 8)}

The active control strategy has been tested on an experimental setup with a single dominant direction of motion. Two versions of the setup have been used, one version with a piezoelectric actuator (PEA) having a suspension mode of $35 \mathrm{~Hz}$ and one version with a voice coil actuator (VCA) having its suspension mode at $17 \mathrm{~Hz}$. These high suspension frequencies are the result of the relatively high support stiffness. The stiffness of the PEA-based setup is 1200 times higher than a soft mount system having its suspension frequency at $1 \mathrm{~Hz}$. The VCA-based setup has a stiffness that is 300 times higher than this soft mount's stiffness.

Table 9.1 lists the best performance results achieved during the control experiments. The RMS acceleration levels measured at a validation sensor on the setup are compared for open loop (OL), closed loop (FB) and feedforward controlled (FF) experiments on both setups. The listed acceleration levels are proportional to the internal deformation of the machine, with the scale factor being $6.7 \mathrm{~nm} /\left(\mathrm{mm} / \mathrm{s}^{2}\right)$.

During the control experiments, both setups have been excited by the same excitation signal. The resulting floor acceleration level is $20 \mathrm{~mm} / \mathrm{s}^{2} \mathrm{RMS}$ $(0-1600 \mathrm{~Hz})$ for the PEA-based setup. For the VCA-based setup, a floor acceleration level of $17 \mathrm{~mm} / \mathrm{s}^{2}$ RMS is obtained. The ratios of the validation acceleration signal to the floor acceleration are also presented in decibels.

In comparison to an (idealized) state-of-the-art soft mount isolation system (1 Hz suspension frequency, 70\% skyhook damping and $-60 \mathrm{~dB}$ high frequency transmissibility limit), the residual machine acceleration levels for the PEA-based and VCA-based setup are higher by a factor of ten and five.

Although the achieved performance of the active hard mount system is not comparable to state-of-the-art soft mount systems yet, the results are encouraging, especially when taking into account the much larger support stiffness.

Table 9.1: Overview of experimental results for the PEA-based and VCAbased setup: open loop (OL), feedback (FB) and feedforward (FF) validation acceleration levels $\left(\ddot{x}_{2}\right)$

\begin{tabular}{|c|c|c|c|c|c|c|}
\hline & \multicolumn{2}{|c|}{ OL } & \multicolumn{2}{c|}{ FB } & \multicolumn{2}{c|}{ FF } \\
\cline { 2 - 7 } & $\left(\mathrm{mm} / \mathrm{s}^{2} \mathrm{a}\right)$ & $\left(\mathrm{dB}^{\mathrm{b}}\right)$ & $\left(\mathrm{mm} / \mathrm{s}^{2}\right)$ & $(\mathrm{dB})$ & $\left(\mathrm{mm} / \mathrm{s}^{2}\right)$ & $(\mathrm{dB})$ \\
\hline PEA & 51 & 8.1 & 4.9 & -12 & 1.7 & -21 \\
\hline VCA & 10 & -4.6 & 1.7 & -20 & 0.8 & -27 \\
\hline
\end{tabular}

a All acceleration levels are expressed in (total) RMS $(0-1600 \mathrm{~Hz})$

${ }^{b}$ Decibels relative to open loop floor acceleration level 
For both setups, the most important factors that limit the vibration isolation performance are found to be:

\section{- Limited actuator signal range:}

The control bandwidth of the feedforward controller can not be extended to lower frequencies, due to the required large gain at lower frequencies. Due to this large gain, the low frequency noise in the reference sensor signal would result in a large drift of the actuator signal and consequently actuator saturation and instability of the adaptation algorithm.

- Mismatched error signal:

The error signal used for adaptation of the feedforward controller (the acceleration $\ddot{x}_{1}$ ) and the true performance signal (the structural deformation) no longer match at frequencies above approximately $150 \mathrm{~Hz}$. As a result, the feedforward control action does not result in an improvement over the feedback control performance at these high frequencies.

- Non-minimum phase zeros in the tertiary path $^{1}$ :

Due to the off-centre placement of the reference sensor on the floor body, a non-minimum phase anti-resonance occurs at $160 \mathrm{~Hz}$ in the tertiary path. As a result, the feedforward controller can not improve the vibration isolation performance at this frequency.

- Poorly damped anti-resonance in the secondary path ${ }^{2}$ :

At the (poorly damped) anti-resonance in the secondary path at $62 \mathrm{~Hz}$, a notch filter is used to stop the control action at this frequency. Otherwise, the actual performance (in terms of the structural deformation) would deteriorate. With the notch filter, at least the performance is not deteriorated over the feedback control performance.

- Parasitic stiffness effects:

Some of the vibration energy is transferred from the shaker (the disturbance source) through parasitic transfer paths (through the setup base plate and the linear guidance flexures). The resulting motion (e.g. tilting modes) can not be controlled by the actuator.

- Phase lag due to delays:

The anti-aliasing filters, the DA-conversion and the computational delay introduce a significant phase lag at frequencies above $100 \mathrm{~Hz}$. As a result, the feedforward controller reacts increasingly "sluggish" to high-frequency floor vibrations and the achievable performance is reduced at higher frequencies. For the feedback controller, the phase lag (combined with high frequency resonances) poses a limit on the achievable control bandwidth.

1 Transfer path from shaker to reference sensor

2 Transfer path from actuator to error sensor 


\subsubsection{Feedback control (chapter 4)}

The feedback controller is designed to add skyhook damping to the suspension resonance mode as well as active damping to the (relevant) structural resonance modes. The feedback law is derived from Direct Velocity Feedback using collocated actuator/sensor pairs, which possesses excellent stability robustness properties.

The ratio of the structural resonance frequency to the associated anti-resonance frequency is found to be a key factor in determining the amount of damping that can be achieved in the structural resonance mode. This ratio is determined by the mass distribution in the machine as well as the sensor type and location.

It has been shown that absolute motion sensors are preferred over force sensors and relative motion sensors when both skyhook damping of the suspension mode as well as active damping in the structural mode are to be achieved.

\subsubsection{Feedforward control (chapter 5)}

The feedforward control attempts to compensate for measured floor vibrations by generating anti-forces. It has been shown that an adaptive controller is required, because the optimal controller ${ }^{3}$ requires an accurate model of parts of the system dynamics that can not be identified in practice.

The adaptation algorithm is based on the Filtered-reference LMS (FxLMS) algorithm, which is widely used in Active Noise Control. The FxLMS algorithm attempts to minimize the instantaneous squared value of the measured error signal. Several existing extensions to the FxLMS algorithm are implemented:

- Noise shaping filter: for preventing actuator saturation and shaping the controller performance in the frequency domain

- Preconditioning: for improving the convergence rate of the algorithm

- Swapping of plant and controller: also for improving the convergence rate of the algorithm

The stability of the adaptation algorithm is determined by a user-defined step size and the accuracy of the secondary path model. Although the robustness to model errors can be improved by regularization of the secondary path model and/or leakage, this comes at the expense of (nominal) performance.

Therefore, an accurate model of the secondary path is required. Moreover, an accurate model of the quaternary path ${ }^{4}$ is necessary for internal compensa-

3 Causal Wiener filter

4 Transfer path from actuator to reference sensor 
tion of the floor accelerations due to the feedforward control action. In section 7.3, a subspace-based frequency domain system identification method is discussed that can be used to obtain such accurate models. One of the key factors in the identification is the use of multisine excitation signals, which allows significant noise reduction by averaging the measured data over multiple periods.

When a Finite Impulse Response (FIR) parametrization of the feedforward controller is used, which is commonly used in Active Noise Control applications, the adaptation of the controller weights is a convex optimization problem. However, for poorly damped systems and high sampling frequencies, the FIR parametrization requires a (very) large number of coefficients. Especially for multiple channel applications, the required real-time computational capacity may be a limiting factor for the vibration isolation performance.

It has been shown in this thesis that an Infinite Impulse Response (IIR) parametrization with fixed poles is better suited for vibration isolation problems. For this IIR parametrization, the adaptation algorithm is still convex. Moreover, when the fixed poles are selected carefully, similar vibration isolation performance can be achieved and the computational complexity can be reduced significantly.

\subsubsection{Sensors (section 6.1)}

As mentioned in section 9.1.2, absolute motion sensors are preferred when skyhook suspension damping as well as active structural damping are desired. The most common absolute motion sensor types are accelerometers and geophones.

For accelerometers, it is shown that a trade-off exists between the sensitivity and bandwidth. The trade-off is inherent to all accelerometers due to the measurement principle on which the sensors are based.

Because geophones measure velocity instead of acceleration, their effective noise level (in terms of acceleration) is remarkably low near the sensor's principal resonance frequency. However, it is also inherent to the inductive measurement principle that the high frequency (and very low frequency) response of geophones is worse compared to accelerometers.

It is also shown that all absolute motion sensors are sensitive to the so-called tilt-to-horizontal coupling effect. As a result, it is impossible for accelerometers to distinguish between translational acceleration and a rotational acceleration with respect to Earth's gravitational field. A geophone acts as a rotational velocity sensor below the sensor's principal resonance frequency and as a translational velocity sensor above this frequency. It is advisable to include this sensitivity to tilt in the dynamic models that are used for controller design. 
It is shown that the sensor requirements for a hard mount vibration isolation system are very stringent, in terms of (effective) noise level and bandwidth. Several sensors have been evaluated for their suitability, but none have been found to meet the requirements over the entire frequency range.

\subsubsection{Actuators (section 6.2)}

Two types of actuators have been evaluated for use in the active hard mount isolation system:

\section{- Piezoelectric stack actuators (PEA):}

The inherently high stiffness of piezoelectric actuators must be reduced for most machines, because the suspension frequency would otherwise become too high. In the experimental setup this has been achieved by adding a compliant component in series with the piezoelectric actuator. The stiffness reduction also reduces the effects of the noise injected by the DA-converter and PEA-amplifier noise to a negligible level. However, the required input signals to generate the required forces are increased.

When operated in open loop (voltage-controlled), the piezoelectric actuator suffers from hysteresis. For vibration isolation applications, the dominant effect of hysteresis is an effective reduction of the actuator's force capacity, requiring larger input signals. Charge controlled or position controlled operation of the piezoelectric actuator can reduce the hysteresis, and may therefore be preferred. For the PEA-based setup, the increased required input signals due to the stiffness reduction and hysteresis have made the output range of the DA-converter a limiting factor for the vibration isolation performance.

- Voice coil actuators (VCA):

The voice coil actuator has no inherent stiffness, allowing more freedom in the mechanical design. Moreover, the hysteresis effect in the voice coil actuator is negligible.

On the other hand, it is shown that the VCA-amplifier noise is the dominant contributor to the machine acceleration level at high frequencies. This noise contribution may be reduced by selecting an actuator/amplifier combination that is better tuned to the particular application as the current actuator/amplifier are more powerful than strictly required.

It is shown that open loop operation (voltage mode) of the VCAamplifier is preferred over closed loop operation (current mode), because the effective noise level is reduced by approximately a factor two. Moreover, in open loop operation, the back-emf results in increased damping of the suspension mode. 
Even though the piezoelectric actuator and the voice coil actuator that have been used in the control experiments have their disadvantages, the active control system has been able to significantly improve the vibration isolation performance in both cases. Therefore, no definitive conclusion on the best actuator type for vibration isolation problems can be drawn. However, it is expected that voice coil actuators are better suited for applications involving relatively lightweight machines, whereas piezoelectric actuators might be a viable alternative for heavier machines.

\subsubsection{Mechanical design (sections 2.5, 3.2, 3.2.3 and 7.2)}

From the experimental results and a theoretical analysis, the following guidelines for the mechanical design of hard mount vibration isolation systems have been found:

\section{- Actuator and sensor placement:}

By connecting the actuators and sensors rigidly and close together and carefully aligning them, the dynamics involved in the feedback loop will exhibit collocated behaviour over a large frequency range. This collocation simplifies the feedback control design and results in a robustly stable feedback loop.

Moreover, if the sensors and actuators are not properly aligned, the secondary path or tertiary path may have non-minimum phase behaviour. It has been shown that such non-minimum phase behaviour results in a reduced feedforward control performance at the associated frequencies.

- Exact constraint design:

Significant parasitic stiffness in the hard mount design can lead to undesired deformations due to e.g. thermal loads. Moreover, the parasitic stiffness results in vibration transfer paths that can not (directly) be compensated for by the feedforward controller. From an analysis of a planar model, it is found that the ratio of principal stiffness to parasitic stiffness should be at least 100 for each mount. If this requirement fulfilled, parasitic stiffness is expected to have a negligible effect on the achievable vibration isolation performance.

- Support stiffness:

Both experimental setups have been designed with relatively high support stiffness. However, the exact values of the support stiffness have been chosen rather arbitrarily. In practice, the support stiffness must be chosen with care as it offers a trade-off between floor vibration isolation and robustness against low-frequency direct disturbances. Its value should therefore be chosen based on the expected floor vibration and direct disturbance levels. 
Moreover, as the required feedforward compensation effort is approximately proportional to the support stiffness, the floor vibration isolation problem is less demanding for lower values of the support stiffness. It is therefore advised to choose the support stiffness not higher than strictly necessary.

\subsection{Recommendations for further research}

From the results that have been obtained in this thesis, several topics for further research are distilled. These suggestions are discussed below:

\section{- Sensor blending:}

The sensor noise level is an important limiting factor in the achievable vibration isolation performance, especially the low frequency noise in the reference sensor. It has also been shown that no sensor exists that meets the requirements over the entire frequency range of interest.

Therefore, it should be investigated whether a combination of sensors can be used for different frequency ranges. Then, an adequate sensor can be chosen for each frequency range. Most likely, such a blended sensor signal should combine a displacement sensor for low frequencies $(f \ll 1 \mathrm{~Hz})$, a geophone or seismic accelerometer for the middle frequency range $(\approx 1 \leq f \leq 100 \mathrm{~Hz})$ and accelerometers and/or force sensors at high frequencies $(f>100 \mathrm{~Hz})$.

Besides offering a superior overall signal quality, it may also be possible to construct from these combined signals an error signal that better represents the actual performance signal for use in the adaptive feedforward controller. By using such a reconstructed error signal, the vibration isolation performance may be improved. The blending of these sensor signals can be straightforward (using low- and high-pass filters), but it may also be possible to use more sophisticated techniques like Kalman filtering.

Moreover, by combining force and acceleration (or velocity) measurements an approximate minimization of the transmitted power is also possible [Beijers (2005)].

- Pole selection of the IIR parametrization:

The performance that is achieved by the feedforward controller using the Infinite Impulse Response parametrization is highly dependent on the pole selection. In this thesis, the poles have been selected as a subset of the poles of the Causal Wiener filter. In practice, this Causal Wiener filter can not be computed and another method for finding good pole locations must be developed. 
Information on the pole locations could be extracted from detailed system models and the system identification results, but experimental tuning of the filter pole locations may also be required.

A combination of IIR poles and pure delays (FIR) may also be interesting, as it has been shown that the FIR filter is better capable of improving the performance at high frequencies $(f>100 \mathrm{~Hz})$.

- Improvement of high-frequency passive isolation:

It has been observed that for the current setup the floor vibration isolation performance above $150 \mathrm{~Hz}$ can not be improved by the active control system. Although it may be possible to improve the control bandwidth, a mechanical design may also be considered that maintains sufficient (static) suspension stiffness, but achieves better roll-off at high frequencies (better than $-40 \mathrm{~dB} /$ decade). If such a design can be realized, the passive floor vibration isolation at high frequencies may be sufficient and no additional active control is required in this frequency range.

- Efficient implementation of the adaptive algorithms:

It has been shown that the IIR parametrization of the feedforward controller leads to a reduction in the computational requirements for the real-time processor. In view of the extension of the research to a multiple channel setup, it remains to be seen whether the implementation using the IIR parametrization is sufficiently efficient. To reduce the computational requirements, block adaptive algorithms may be considered, see e.g. [Sayed (2003), Ch. 10]. These algorithms only compute an update at every $N^{\text {th }}$ sample, thereby reducing the computational time required per sample.

It may also be worthwhile to investigate whether the control algorithms can be implemented on faster real-time platforms, based on e.g. field-programmable gate arrays (FPGA) or graphical processor units (GPU), see e.g. [Wesselink (2009)].

- Extension to multiple channels:

The experimental results have been obtained on a simplified experimental setup with only one dominant direction of motion. An important step in the development of the active hard mount vibration isolation concept is to test the feedback and feedforward control strategies on an experimental setup that allows motion in all directions. 



\section{Appendix A}

\section{System models}

In this appendix, a basic system model that is used to illustrate the design trade-offs in vibration isolation is presented in section A.1. Moreover, a more elaborate model for the experimental setup is presented in section A.2.

\section{A.1 Basic system model}

In this section, a basic model is described which is used to illustrate the design trade-offs that exist for vibration isolation systems. The first section is dedicated to the model's equations of motion and the resulting transfer functions. Then, the sensitivity of the transfer functions to the model parameters is discussed in section A.1.3, resulting in some basic design guidelines for highprecision equipment and their supports.

\section{A.1.1 Equations of motion}

In figure A.1a, a basic system model is presented that still describes many of the relevant phenomena that occur in vibration isolation problems. The model uses lumped masses, linear springs and viscous dampers to describe the dynamics of the machine, the mount and the floor. All bodies are restricted to translate only in one direction. The machine has one internal degree of freedom. The mount is modelled as the parallel connection of the spring $k_{1}$ and the viscous damper $d_{1}$. The floor is modelled as a mass-spring-damper system. Hence, the compliance of the floor, as well as its dominant vibration mode, can be taken into account. Several disturbance forces $\left(F_{d_{0}}, F_{d_{1}}, F_{d_{2}}\right)$ are shown that can excite the machine masses and the floor. 


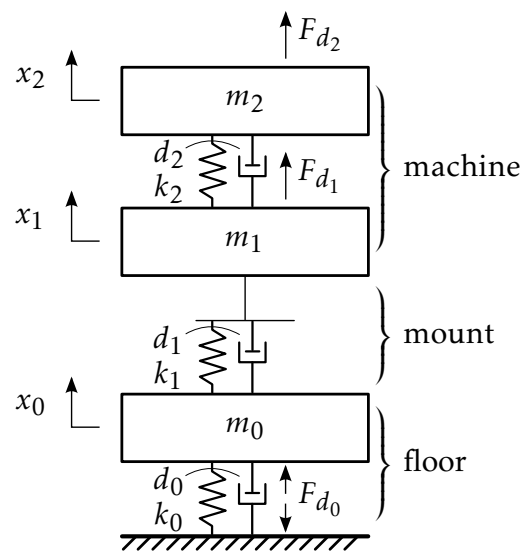

(a) Passive vibration isolation model

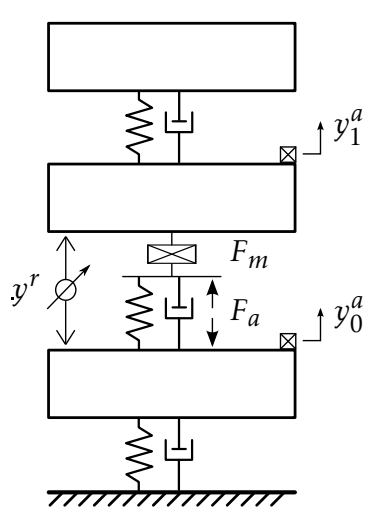

(b) Additional elements in the active vibration isolation model

Figure A.1: Elementary models of vibration isolation problems

Figure A.1b shows additional elements that may be present in active vibration isolation systems, i.e. a force actuator $F_{a}$ and several sensors $F_{m}, y^{r}, y_{0}^{a}$ and $y_{1}^{a}$. The expressions for the measurement signals are given in equations (A.1)(A.4). The absolute motion measurements are expressed in terms of displacement.

$$
\begin{aligned}
& F_{m}(t)=F_{a}(t)-k_{1}\left(x_{1}(t)-x_{0}(t)\right)-d_{1}\left(\dot{x}_{1}(t)-\dot{x}_{0}(t)\right) \\
& y^{r}(t)=x_{1}(t)-x_{0}(t) \\
& y_{0}^{a}(t)=x_{0}(t) \\
& y_{1}^{a}(t)=x_{1}(t)
\end{aligned}
$$

The equations of motion for this system are given by equations (A.5). Here, $x$ is the vector of mass displacements and $f$ is the vector of external forces, see equations (A.6) and (A.7). The input selection matrix $\boldsymbol{B}$ is given by equation (A.8). The mass, damping and stiffness matrices $\boldsymbol{M}, \boldsymbol{D}$ and $\boldsymbol{K}$ are specified in equations (A.9)-(A.11).

$$
\begin{aligned}
& \boldsymbol{M} \ddot{\boldsymbol{x}}(t)+\boldsymbol{D} \dot{\boldsymbol{x}}(t)+\boldsymbol{K} \boldsymbol{x}(t)=\boldsymbol{B} \boldsymbol{f}(t) \\
& \boldsymbol{x}(t)=\left[\begin{array}{lll}
x_{0}(t) & x_{1}(t) & x_{2}(t)
\end{array}\right]^{\mathrm{T}} \\
& \boldsymbol{f}(t)=\left[\begin{array}{llll}
F_{d_{0}}(t) & F_{d_{1}}(t) & F_{d_{2}}(t) & F_{a}(t)
\end{array}\right]^{\mathrm{T}}
\end{aligned}
$$




$$
\begin{aligned}
\boldsymbol{B} & =\left[\begin{array}{cccc}
1 & 0 & 0 & -1 \\
0 & 1 & 0 & 1 \\
0 & 0 & 1 & 0
\end{array}\right] \\
\boldsymbol{M} & =\left[\begin{array}{ccc}
m_{0} & 0 & 0 \\
0 & m_{1} & 0 \\
0 & 0 & m_{2}
\end{array}\right] \\
\boldsymbol{D} & =\left[\begin{array}{ccc}
d_{0}+d_{1} & -d_{1} & 0 \\
-d_{1} & d_{1}+d_{2} & -d_{2} \\
0 & -d_{2} & d_{2}
\end{array}\right] \\
\boldsymbol{K} & =\left[\begin{array}{ccc}
k_{0}+k_{1} & -k_{1} & 0 \\
-k_{1} & k_{1}+k_{2} & -k_{2} \\
0 & -k_{2} & k_{2}
\end{array}\right]
\end{aligned}
$$

Commonly, the machine response is described in terms of the displacement $x_{1}$ and the deformation $\Delta x \equiv x_{2}-x_{1}$. This can be achieved by a change in variables as indicated in equation (A.12). Here, $\boldsymbol{q}(t)$ are called generalized coordinates. In order to retain the symmetry in the system matrices, the equations of motion are multiplied from the left by $V^{\mathrm{T}}$, see equation (A.13).

$$
\begin{aligned}
& \boldsymbol{x}(t)=\left[\begin{array}{lll}
1 & 0 & 0 \\
0 & 1 & 0 \\
0 & 1 & 1
\end{array}\right]\left[\begin{array}{c}
x_{0}(t) \\
x_{1}(t) \\
\Delta x(t)
\end{array}\right]=\boldsymbol{V} \boldsymbol{q}(t) \\
& \underbrace{\boldsymbol{V}^{\mathrm{T}} \boldsymbol{M} \boldsymbol{V}}_{\overline{\boldsymbol{M}}} \ddot{\boldsymbol{q}}(t)+\underbrace{\boldsymbol{V}^{\mathrm{T}} \boldsymbol{D} \boldsymbol{V}}_{\overline{\boldsymbol{D}}} \dot{\boldsymbol{q}}(t)+\underbrace{\boldsymbol{V}^{\mathrm{T}} \boldsymbol{K} \boldsymbol{V}}_{\overline{\boldsymbol{K}}} \boldsymbol{q}(t)=\underbrace{\boldsymbol{V}^{\mathrm{T}} \boldsymbol{B}}_{\overline{\boldsymbol{B}}} \boldsymbol{f}(t)
\end{aligned}
$$

Equation (A.14) expresses the equations of motion in the Laplace domain. The first term on the right-hand side denotes the forced excitation, whereas the latter two terms are related to the response to non-zero initial conditions.

$$
\left[\overline{\boldsymbol{M}} s^{2}+\overline{\boldsymbol{D}} s+\overline{\boldsymbol{K}}\right] \boldsymbol{Q}(s)=\overline{\boldsymbol{B}} \boldsymbol{F}(s)+[\overline{\boldsymbol{M}} s+\overline{\boldsymbol{D}}] \boldsymbol{q}(0)+\overline{\boldsymbol{M}} \dot{\boldsymbol{q}}(0)
$$




\section{A.1.2 Transfer functions}

The transfer function matrix relating the generalized coordinates to the force inputs is given by equation (A.15). For low order systems (typically $\leq 3$ ), the inverse matrix in equation (A.15) can be easily calculated from equation (A.16), where $\operatorname{adj}(\boldsymbol{A})$ is the adjugate or classical adjoint of a matrix $\boldsymbol{A}$ and $\operatorname{det}(\boldsymbol{A})$ is its determinant.

$$
\begin{aligned}
\frac{\boldsymbol{Q}(s)}{\boldsymbol{F}(s)} & =\left[\overline{\boldsymbol{M}} s^{2}+\overline{\mathbf{D}} s+\overline{\boldsymbol{K}}\right]^{-1} \overline{\boldsymbol{B}} \\
\boldsymbol{A}^{-1} & =\frac{\operatorname{adj}(\boldsymbol{A})}{\operatorname{det}(\boldsymbol{A})} \\
\operatorname{adj}(\boldsymbol{A})_{i j} & =(-1)^{i+j} M_{j i}
\end{aligned}
$$

The $(i, j)$-entry of the adjugate is defined in equation (A.17). Here, $M_{j i}$ is a minor of $A$, i.e. the determinant of the sub-matrix of $A$ that results when the $j^{\text {th }}$ row and the $i^{\text {th }}$ column are removed.

Please note that adj $(A)_{i j}$ can be interpreted as the numerator polynomial $N_{i j}(s)$ of the transfer function $\frac{Q_{i}(s)}{F_{j}(s)}$. Moreover, $\operatorname{det}(\boldsymbol{A})$ is the denominator polynomial of the transfer functions.

\section{Full model}

When evaluating the full model of equation (A.14), the transfer function matrix is given by equation (A.18). The denominator polynomial is given in equation (A.19). Note that, for ease of notation, the dynamic stiffnesses $\tilde{k}_{i}$ are introduced, as shown in equation (A.20). The numerator polynomials are listed in equations (A.21)-(A.32).

$$
\begin{aligned}
\frac{\boldsymbol{Q}(s)}{\boldsymbol{F}(s)}= & \frac{1}{p(s)}\left[\begin{array}{ccc}
N_{11}(s) & \ldots & N_{14}(s) \\
\vdots & \ddots & \vdots \\
N_{31}(s) & \ldots & N_{34}(s)
\end{array}\right] \\
p(s)= & \left(m_{0} s^{2}+\tilde{k}_{0}+\tilde{k}_{1}\right)\left(m_{1} s^{2}+\tilde{k}_{1}+\tilde{k}_{2}\right)\left(m_{2} s^{2}+\tilde{k}_{2}\right) \\
& \quad-\tilde{k}_{2}^{2}\left(m_{0} s^{2}+\tilde{k}_{0}+\tilde{k}_{1}\right)-\tilde{k}_{1}^{2}\left(m_{2} s^{2}+\tilde{k}_{2}\right) \\
\tilde{k}_{i}= & k_{i}+d_{i} s, \quad i=0,1,2
\end{aligned}
$$




$$
\begin{aligned}
& N_{11}(s)=\left(m_{1} s^{2}+\tilde{k}_{1}+\tilde{k}_{2}\right)\left(m_{2} s^{2}+\tilde{k}_{2}\right)-\tilde{k}_{2}^{2} \\
& N_{12}(s)=\tilde{k}_{1}\left(m_{2} s^{2}+\tilde{k}_{2}\right) \\
& N_{13}(s)=\tilde{k}_{1} \tilde{k}_{2} \\
& N_{14}(s)=-s^{2}\left(m_{1} m_{2} s^{2}+\tilde{k}_{2}\left(m_{1}+m_{2}\right)\right) \\
& N_{21}(s)=N_{12}(s) \\
& N_{22}(s)=\left(m_{0} s^{2}+\tilde{k}_{0}+\tilde{k}_{1}\right)\left(m_{2} s^{2}+\tilde{k}_{2}\right) \\
& N_{23}(s)=\tilde{k}_{2}\left(m_{0} s^{2}+\tilde{k}_{0}+\tilde{k}_{1}\right) \\
& N_{24}(s)=\left(m_{0} s^{2}+\tilde{k}_{0}\right)\left(m_{2} s^{2}+\tilde{k}_{2}\right) \\
& N_{31}(s)=-m_{2} s^{2} \tilde{k}_{1} \\
& N_{32}(s)=-m_{2} s^{2}\left(m_{0} s^{2}+\tilde{k}_{0}+\tilde{k}_{1}\right) \\
& N_{33}(s)=\left(m_{0} s^{2}+\tilde{k}_{0}+\tilde{k}_{1}\right)\left(m_{1} s^{2}+\tilde{k}_{1}\right)-\tilde{k}_{1}^{2} \\
& N_{34}(s)=-m_{2} s^{2}\left(m_{0} s^{2}+\tilde{k}_{0}\right)
\end{aligned}
$$

\section{Rheonomic floor motion}

When the floor motion is assumed to be an independent input signal, the equations of motion and the associated transfer functions are slightly altered. In this case, only the lower right $2 \times 2$ blocks of $\boldsymbol{M}, \boldsymbol{D}, \boldsymbol{K}$ and $\boldsymbol{V}$ need to be used. Moreover, the generalized coordinates $Q(\mathrm{~s})$, the force input vector $F(\mathrm{~s})$ and the input selection matrix $\boldsymbol{B}$ are changed, as indicated in equations (A.33)-(A.35).

$$
\begin{aligned}
& \tilde{Q}(s)=\left[\begin{array}{ll}
X_{1}(s) & \Delta X(s)
\end{array}\right]^{\mathrm{T}} \\
& \tilde{\boldsymbol{F}}(s)=\left[\begin{array}{llll}
\tilde{k}_{1} X_{0}(s) & F_{d_{1}}(s) & F_{d_{2}}(s) & F_{a}(s)
\end{array}\right]^{\mathrm{T}} \\
& \tilde{\boldsymbol{B}}=\left[\begin{array}{llll}
1 & 1 & 0 & 1 \\
0 & 0 & 1 & 0
\end{array}\right]
\end{aligned}
$$


As there are three linearly dependent columns in $\tilde{\boldsymbol{B}}$, there are only four transfer functions required to describe the system response, see equation (A.36). The denominator polynomial for this reduced system is expressed in equation (A.37).

$$
\begin{aligned}
& \frac{\tilde{\boldsymbol{Q}}(s)}{\tilde{\boldsymbol{F}}(s)}=\frac{1}{p_{\mathrm{r}}(s)}\left[\begin{array}{llll}
\tilde{N}_{11}(s) & \tilde{N}_{11}(s) & \tilde{N}_{12}(s) & \tilde{N}_{11}(s) \\
\tilde{N}_{21}(s) & \tilde{N}_{21}(s) & \tilde{N}_{22}(s) & \tilde{N}_{21}(s)
\end{array}\right] \\
& p_{\mathrm{r}}(s)=\left(m_{1} s^{2}+\tilde{k}_{1}+\tilde{k}_{2}\right)\left(m_{2} s^{2}+\tilde{k}_{2}\right)-\tilde{k}_{2}^{2}
\end{aligned}
$$

The numerator polynomials are derived using equation (A.17). The results are listed in equations (A.38)-(A.41).

$$
\begin{aligned}
& \tilde{N}_{11}(s)=\left(m_{2} s^{2}+\tilde{k}_{2}\right) \\
& \tilde{N}_{12}(s)=\tilde{k}_{2} \\
& \tilde{N}_{21}(s)=-m_{2} s^{2} \\
& \tilde{N}_{22}(s)=\left(m_{1} s^{2}+\tilde{k}_{1}\right)
\end{aligned}
$$

These transfer functions define the compliances $C_{i}(s)$ and the deformabilities $D_{i}(s)$, as shown in equations (A.42)-(A.45). Lastly, the transmissibility $T(s)$ and deformation transmissibility $T_{d}(s)$ are defined by equations (A.46) and (A.47). Please note that the floor motion input is expressed in terms of acceleration.

$$
\begin{aligned}
& C_{1}(s) \equiv \frac{X_{1}(s)}{F_{d_{1}}(s)}=\frac{\left(m_{2} s^{2}+\tilde{k}_{2}\right)}{p_{\mathrm{r}}(s)} \\
& C_{2}(s) \equiv \frac{X_{1}(s)}{F_{d_{2}}(s)}=\frac{\tilde{k}_{2}}{p_{\mathrm{r}}(s)} \\
& D_{1}(s) \equiv \frac{\Delta X(s)}{F_{d_{1}}(s)}=-\frac{m_{2} s^{2}}{p_{\mathrm{r}}(s)} \\
& D_{2}(s) \equiv \frac{\Delta X(s)}{F_{d_{2}}(s)}=\frac{\left(m_{1} s^{2}+\tilde{k}_{1}\right)}{p_{\mathrm{r}}(s)} \\
& T(s) \equiv \frac{\ddot{X}_{1}(s)}{\ddot{X}_{0}(s)}=\frac{X_{1}(s)}{X_{0}(s)}=\frac{\tilde{k}_{1}\left(m_{2} s^{2}+\tilde{k}_{2}\right)}{p_{\mathrm{r}}(s)} \\
& T_{d}(s) \equiv \frac{\Delta X(s)}{\ddot{X}_{0}(s)}=\frac{\Delta X(s)}{s^{2} X_{0}(s)}=-\frac{\tilde{k}_{1} m_{2}}{p_{\mathrm{r}}(s)}
\end{aligned}
$$


For later use, it is pointed out that the deformation transmissibility (A.47) is related to the transfer function $\ddot{X}_{2}(s) / \ddot{X}_{0}(s)$ as shown in equation (A.48). In case the damping $d_{2}$ can be neglected (which is usually the case), the deformation due to floor vibrations can be conveniently evaluated by scaling the measured acceleration $\ddot{x}_{2}(t)$ by $-m_{2} / k_{2}$.

$$
\frac{\ddot{X}_{2}(s)}{\ddot{X}_{0}(s)}=\frac{\tilde{k}_{1} \tilde{k}_{2}}{p_{r}(s)}=-\frac{\tilde{k}_{2}}{m_{2}} \frac{\Delta X(s)}{\ddot{X}_{0}(s)}
$$

\section{A.1.3 Parameter sensitivity}

The transfer functions of equations (A.42)-(A.47) depend on the mechanical properties of the machine $\left(m_{1}, m_{2}, k_{2}\right.$ and $\left.d_{2}\right)$ as well as on those of the support $\left(k_{1}\right.$ and $\left.d_{1}\right)$. Even though the former parameters are usually given design constraints for a vibration isolation system design, it is interesting to explore the effect of these machine design parameters on the machine's sensitivity to the various disturbance sources.

\section{Machine parameters}

In figure A.2, the (deformation) transmissibility, compliances and deformabilities are depicted for an exemplary system. The system's suspension resonance frequency $f_{\text {susp }}$ is $5 \mathrm{~Hz}$ and the internal resonance frequency $f_{\text {int }}$ is 100 Hz. The damping ratio of the suspension frequency is $1 \%$. Two values of $d_{2}$ were evaluated, resulting in internal mode damping ratios of $1 \%$ (dashed) and $10 \%$ (solid) respectively.

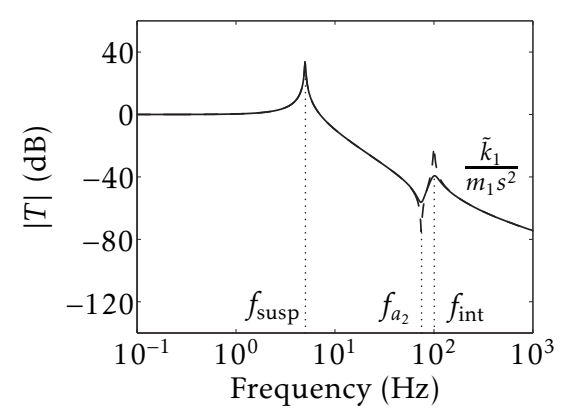

(a) Magnitude frequency response of $T(s)$

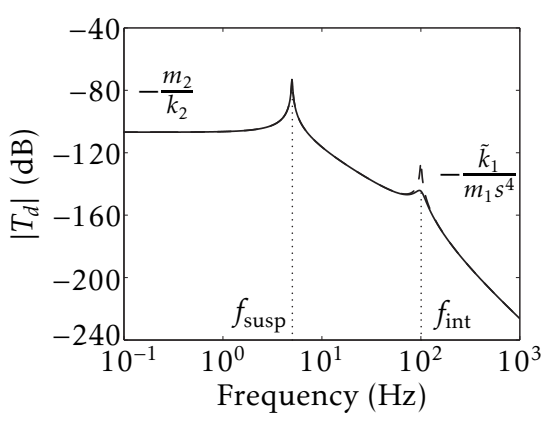

(b) Magnitude frequency response of $T_{d}(s)$

Figure A.2: Magnitude frequency responses of $T(s)$ and $T_{d}(s)$ 


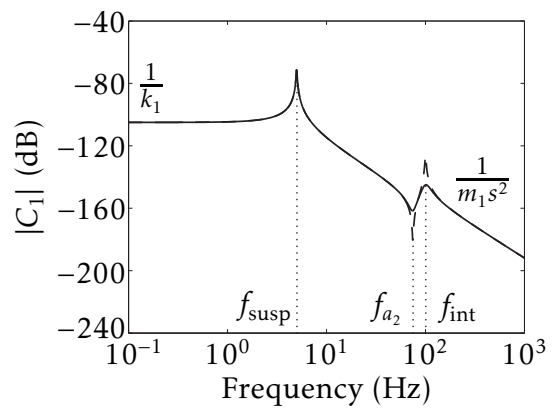

(c) Magnitude frequency response of $C_{1}(s)$

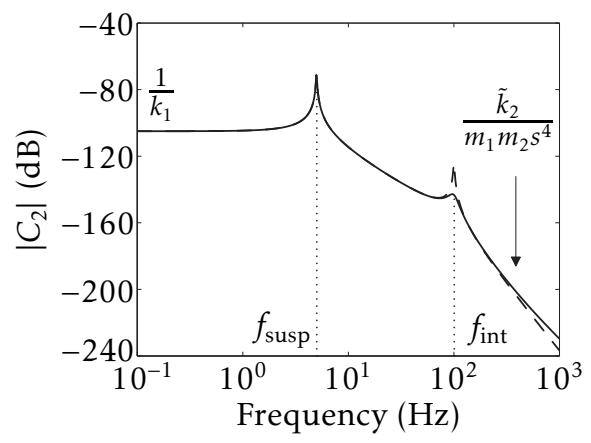

(e) Magnitude frequency response of $C_{2}(s)$

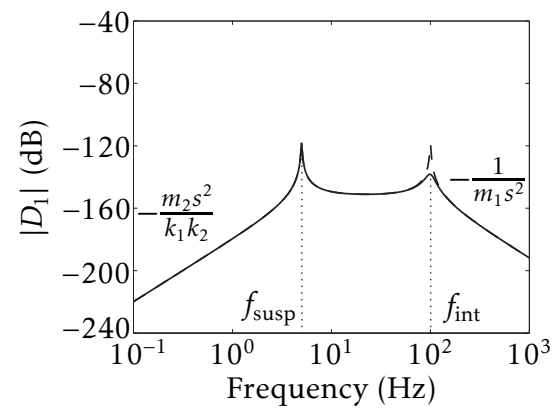

(d) Magnitude frequency response of $D_{1}(s)$

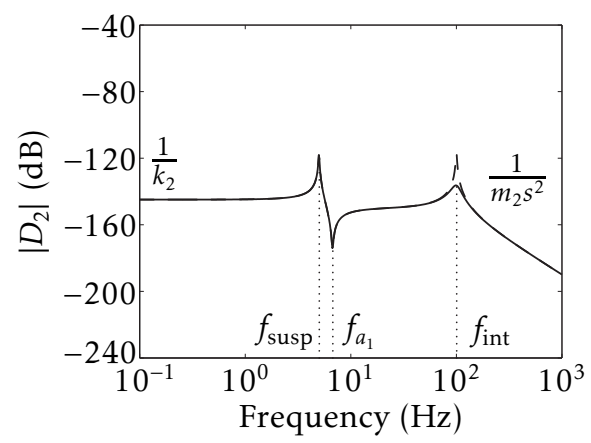

(f) Magnitude frequency response of $D_{2}(s)$

Figure A.2 (cont.): Magnitude frequency responses of $C_{1}(s), D_{1}(s), C_{2}(s)$ and $D_{2}(s)$

The asymptotic behaviour at low and high frequencies is also indicated for the various transfer functions. The indicated antiresonance frequencies $f_{a_{1}}$ and $f_{a_{2}}$ are expressed in equation (A.49) (assuming zero damping).

$$
f_{a_{i}}=\frac{1}{2 \pi} \sqrt{\frac{k_{i}}{m_{i}}}, \quad i=1,2
$$

Based on the indicated asymptotic behaviour in figure A.2, the effects of all six mechanical parameters can be evaluated. Firstly, it is clear from figures (a)(e), that a large value for $m_{1}$ is beneficial at frequencies $f$ above $f_{\text {susp. }}$. Hence, a heavy base frame is preferred. In practice, increasing the frame mass may have some disadvantages. Due to the added mass, resonance frequencies may shift to lower frequencies. When these resonance modes are excited by disturbances, the overall machine accuracy may actually be reduced.

Moreover, the machine will occupy more area and volume and will be more difficult to handle/transport. All these effects add to the production and installation costs of the machine. As a result, a significant increase of the frame mass may not always be an economically viable option. 
Based on figures (b) and (d), a small value of $m_{2}$ reduces the deformation transmissibility $T_{d}$ and the deformability $D_{1}$ for $f<f_{\text {int }}$. However, a large value of $m_{2}$ is required for low sensitivity to $F_{d_{2}}$ at $f>f_{\text {int }}$, according to figures (e) and (f). In many cases, floor vibrations are the dominant disturbance source and, hence, a small value of $m_{2}$ is preferred.

A large value is preferred for $k_{2}$, as can be deduced from figures (b), (d) and (f). It reduces the deformation sensitivity to all disturbance sources at $f<f_{\text {int }}$. The adverse effect of a small value for $k_{2}$ at $f>f_{\text {int }}$ in figure (e) is then taken for granted.

The beneficial effect of high internal damping (a large value for $d_{2}$ ) on the resonance peak at $f_{\text {int }}$ is obvious from all figures (compare dashed and solid lines). There is only a slight, insignificant adverse effect in figure (e) for $f>f_{\text {int }}$. It should be noted that, in practice, it is very difficult to obtain such high internal damping ratios by passive means. Construction elements that allow energy dissipation usually possess nonlinear behaviour (e.g. friction, hysteresis), which introduce some positional uncertainty. This behaviour is in contradiction with the main objective of the mechanical design, which is to assure small, predictable deformations.

Summarizing the above, it can be stated that, in order to reduce the sensitivity to (most) disturbance sources, a lightweight, stiff upper structure design is preferred, with sufficient internal damping and placed on a heavy (rigid) base.

\section{Support parameters}

With respect to the machine support parameters $k_{1}$ and $d_{1}$, the analysis produces more ambivalent results. The effects on the transmissibility $T(s)$ and compliance $C_{1}(s)$ are illustrated in more detail in figure A.3 and A.4.

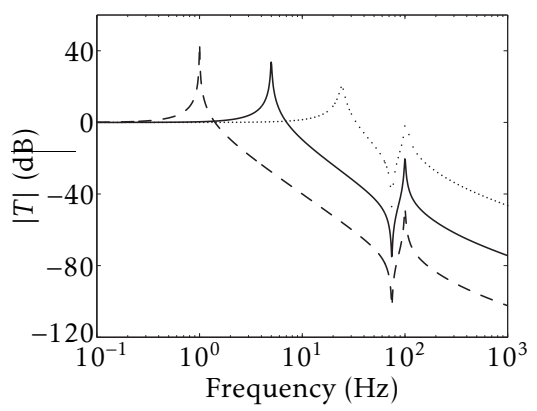

(a) Magnitude frequency response of $T(s)$

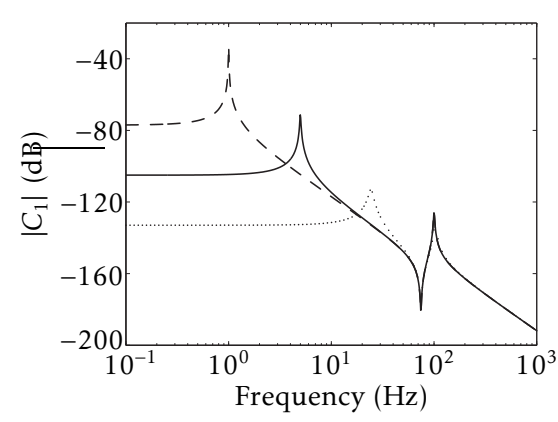

(b) Magnitude frequency response of $C_{1}(s)$

Figure A.3: Effect of $k_{1}$ on the transmissibility $T(s)$ and the compliance $C_{1}(s)$. The value of $k_{1}$ was varied by a factor 25 , resulting in suspension frequencies of $1 \mathrm{~Hz}$ (dashed), $5 \mathrm{~Hz}$ (solid) and $25 \mathrm{~Hz}$ (dotted). 
From figure A.3a, it is observed that a low support stiffness $k_{1}$ is preferred with regard to the transmissibility $T(s)$, whereas a high support stiffness is more beneficial in terms of the compliance $C_{1}(s)$ (see figure A.3b).

It is left as an exercise to the reader to verify that the effect of $k_{1}$ on the deformation transmissibility $T_{d}(s)$ is similar to the effect on the transmissibility $T(s)$, and the effects on the compliance $C_{2}(s)$ and the deformability $D_{1}(s)$ are similar to the effect on the compliance $C_{1}(s)$. There is no significant effect of $k_{1}$ on the deformability $D_{2}(s)$.

As a result of these conflicting requirements, the choice of $k_{1}$ is a design tradeoff, which depends mostly on the expected level of the various disturbances (and, most importantly, on the relative importance of floor vibrations versus direct disturbance forces). In most applications, floor vibrations are the major source of disturbances, and hence a soft mount system is preferred. Unfortunately, the low support stiffness in soft mount systems introduces several disadvantages compared to hard mount systems:

- direct disturbances induce larger displacements and deformations, as well as longer settling times

- the tilt modes of machines with a high centre of mass may become unstable

- the levelling problem due to gravity

To illustrate the levelling problem, equation (A.50) gives the static deflection $\Delta x$ of a (linear) spring due to mass loading in Earth's gravitational field. For a (typical) soft mount suspension resonance frequency of $1 \mathrm{~Hz}$, the static deflection will be $0.25 \mathrm{~m}(!)$. In order to compensate for this sagging, soft mount systems have to be equipped with an additional levelling system, which inherently operates in a very small bandwidth.

$$
\Delta x=-\frac{m g}{k}=-\frac{g}{\left(2 \pi f_{\text {susp }}\right)^{2}}
$$

Another design trade-off exists for the value of $d_{1}$. Figure A.4a and A.4b indicate that the resonance peak at the suspension frequency is reduced by increasing $d_{1}$. However, a large value for $d_{1}$ results in a significantly increased transmissibility above the suspension frequency (see figure A.4a).

Again, it is left to the reader to verify that the effect of $d_{1}$ on $T_{d}(s)$ is similar to the effect on $T(s)$, and the effects on $C_{2}(s), D_{1}(s)$ and $D_{2}(s)$ are similar to the effect on $C_{1}(s)$. 


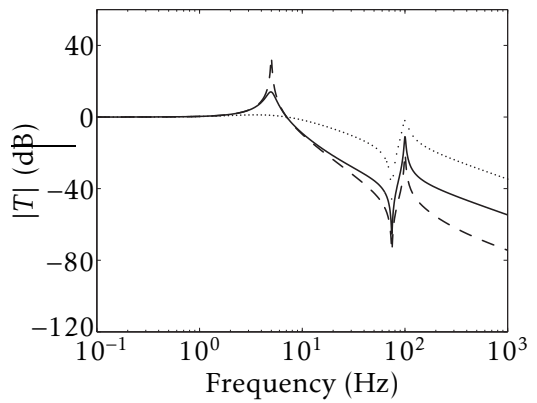

(a) Magnitude frequency response of $T(s)$

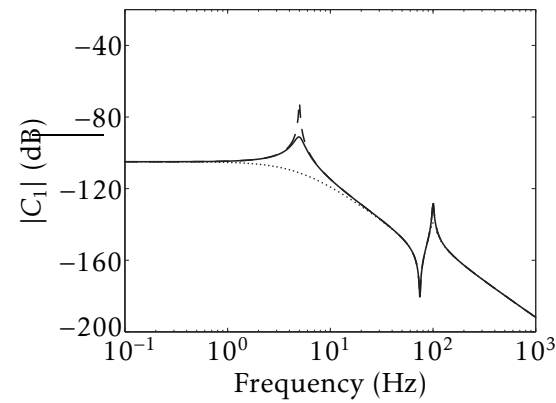

(b) Magnitude frequency response of $C_{1}(s)$

Figure A.4: Effect of $d_{1}$ on the transmissibility $T(s)$ and the compliance $C_{1}(s)$. The value of $d_{1}$ was varied by a factor 10 , resulting in suspension damping ratios of $1 \%$ (dashed), $10 \%$ (solid) and $100 \%$ (dotted).

\section{A.2 Experimental setup models}

In this section, the modelling of the experimental setup is discussed. In section A.2.1, a lumped-parameter, single-directional model of the mechanical subsystem is presented. Then, in section A.2.2, the modelling of the various electrical components is treated briefly.

\section{A.2.1 One-directional model}

Figure A.5 shows the model structure of the experimental setup. This structure is valid for either actuator type (piezoelectric (PEA) or voice coil actuator (VCA)). For a description of the various model elements, see section 7.2.1. Table A.1 lists the model parameters for both setups.

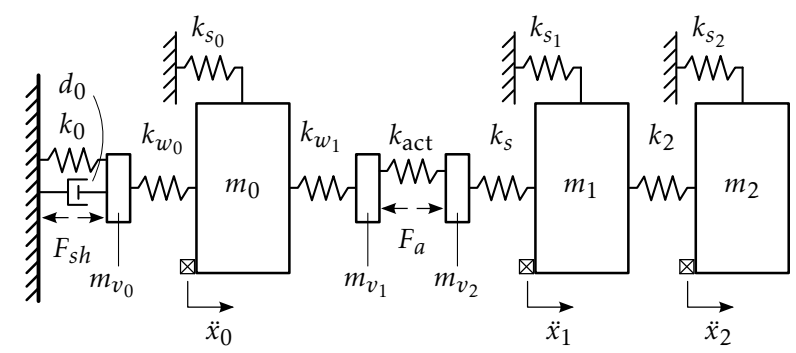

Figure A.5: Lumped parameter model of the experimental setup, for the principal direction of motion 
Table A.1: Model parameters for the experimental setups

\begin{tabular}{|c|c|c|c|}
\hline Parameter & PEA & VCA & Unit \\
\hline$m_{0}$ & 20 & id. & $\mathrm{kg}$ \\
\hline$m_{1}$ & 2.7 & 3 & $\mathrm{~kg}$ \\
\hline$m_{2}$ & 2.7 & id. & $\mathrm{kg}$ \\
\hline$m_{v_{0}}$ & 0.06 & id. & $\mathrm{kg}$ \\
\hline$m_{v_{1}}$ & 0.01 & 0.03 & $\mathrm{~kg}$ \\
\hline$m_{v_{2}}$ & 0.05 & 1 & $\mathrm{~kg}$ \\
\hline$k_{0}$ & $1.2 \cdot 10^{4}$ & id. & $\mathrm{N} / \mathrm{m}$ \\
\hline$d_{0}$ & 90 & id. & $\mathrm{N} \mathrm{s} / \mathrm{m}$ \\
\hline$k_{2}$ & $4.5 \cdot 10^{5}$ & id. & $\mathrm{N} / \mathrm{m}$ \\
\hline$k_{s_{0}}$ & $2.7 \cdot 10^{3}$ & id. & $\mathrm{N} / \mathrm{m}$ \\
\hline$k_{s_{1}}$ & $2.7 \cdot 10^{3}$ & id. & $\mathrm{N} / \mathrm{m}$ \\
\hline$k_{s_{2}}$ & $2.7 \cdot 10^{3}$ & id. & $\mathrm{N} / \mathrm{m}$ \\
\hline$k_{w_{0}}$ & $3 \cdot 10^{6}$ & id. & $\mathrm{N} / \mathrm{m}$ \\
\hline$k_{w_{1}}$ & $3.8 \cdot 10^{6}$ & $2 \cdot 10^{6}$ & $\mathrm{~N} / \mathrm{m}$ \\
\hline$k_{\text {act }}$ & $1.2 \cdot 10^{7}$ & $6.4 \cdot 10^{4}$ & $\mathrm{~N} / \mathrm{m}$ \\
\hline$k_{s}$ & $3 \cdot 10^{5}$ & $1.3 \cdot 10^{8}$ & $\mathrm{~N} / \mathrm{m}$ \\
\hline
\end{tabular}

The dynamical behaviour of the setup can be described in the frequency domain by six transfer functions, as defined in equation (A.51). Given the model parameters in table A.1, these transfer functions are easily computed using the method discussed in appendix A.1.2. The resulting frequency response of $\boldsymbol{H}_{m}(s)$ is shown in figure A.6.

$$
\boldsymbol{H}_{m}(s)=\left[\begin{array}{ll}
H_{11}(s) & H_{12}(s) \\
H_{21}(s) & H_{22}(s) \\
H_{31}(s) & H_{32}(s)
\end{array}\right] \equiv\left[\begin{array}{ll}
\frac{\ddot{X}_{0}(s)}{F_{s h}(s)} & \frac{\ddot{X}_{0}(s)}{F_{a}(s)} \\
\frac{\ddot{X}_{1}(s)}{F_{s h}(s)} & \frac{\ddot{X}_{1}(s)}{F_{a}(s)} \\
\frac{\ddot{X}_{2}(s)}{F_{s h}(s)} & \frac{\ddot{X}_{2}(s)}{F_{a}(s)}
\end{array}\right]
$$



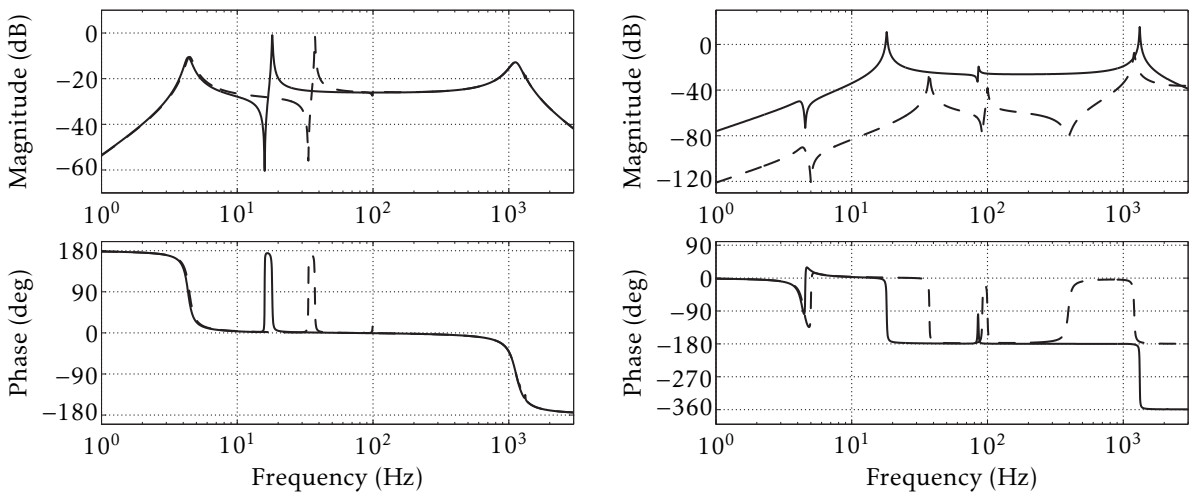

(a) $H_{11}(s)=\ddot{X}_{0}(s) / F_{s h}(s)$

(b) $H_{12}(s)=\ddot{X}_{0}(s) / F_{a}(s)$
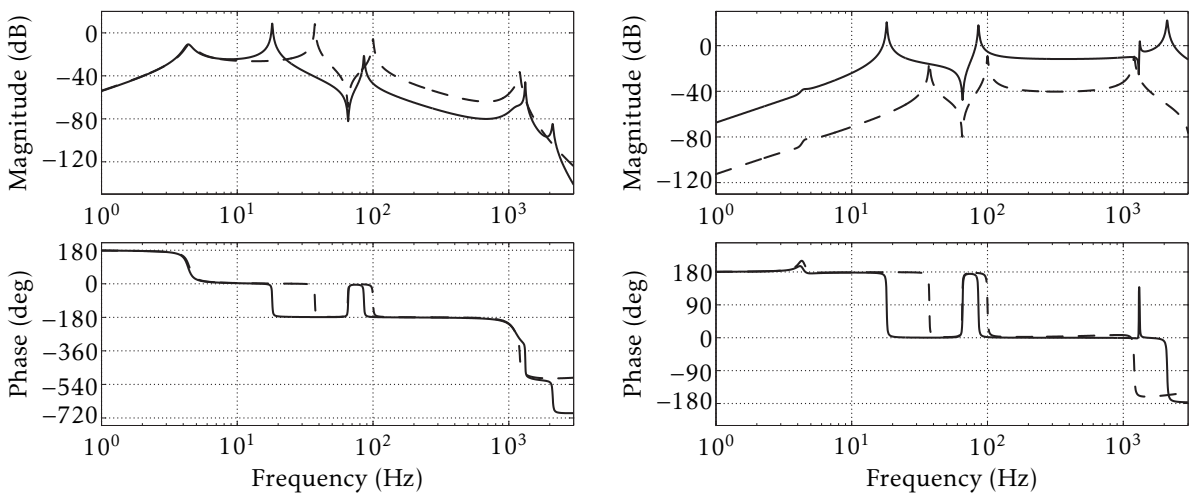

(c) $H_{21}(s)=\ddot{X}_{1}(s) / F_{s h}(s)$

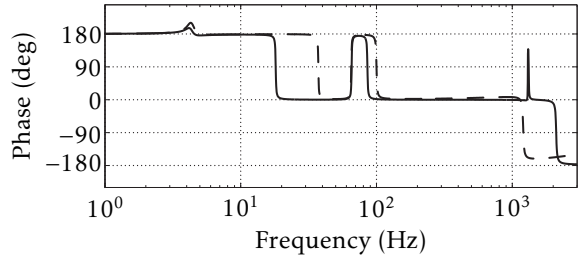

(d) $H_{22}(s)=\ddot{X}_{1}(s) / F_{a}(s)$
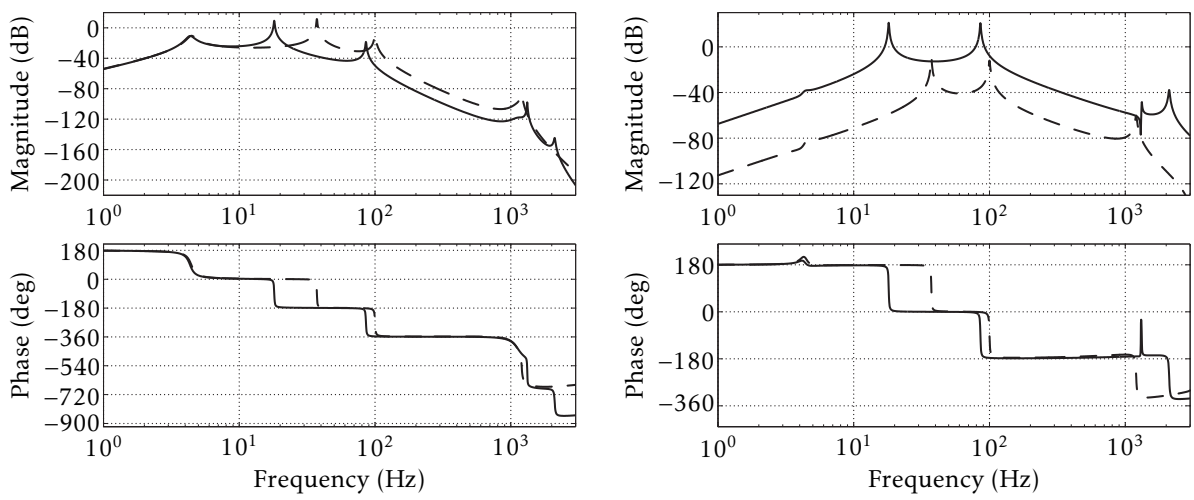

(e) $H_{31}(s)=\ddot{X}_{2}(s) / F_{s h}(s)$

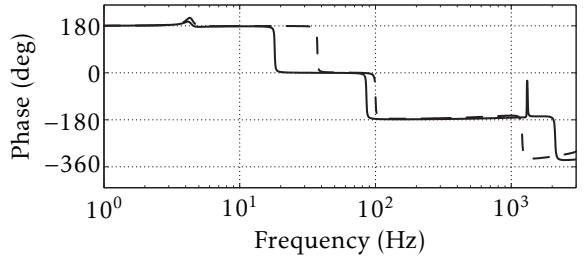

(f) $H_{32}(s)=\ddot{X}_{2}(s) / F_{a}(s)$

Figure A.6: Bode diagrams of the experimental setup models [eqn. (A.51)], _ : setup with VCA; - - : setup with PEA 


\section{A.2.2 Signal conditioning and power electronics}

A block diagram showing the interconnection of the mechanical system and the sensor and actuator electronics is shown in figure A.7. The input signals $u_{s h}$ and $u_{a}$ represent the discrete driving signals for the shaker and the (PEA or VCA) actuator. The output signals $y_{0}, y_{1}$ and $y_{2}$ are sampled versions of the sensor signals, which represent the accelerations of the floor mass $m_{0}$, the first machine mass $m_{1}$ and the second machine mass $m_{2}$ respectively.

An overview of the relevant parameters for the electrical system components is presented in table A.2.

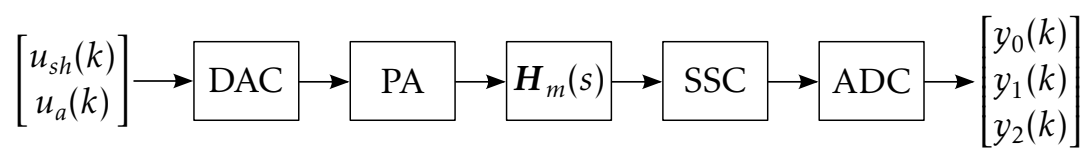

Figure A.7: Block diagram showing the interconnection of the mechanical system and the electrical components

$D A C$ : DA-converters (dSPACE DS2102)

$P A$ : power amplifiers and actuator electronics

$\boldsymbol{H}_{m}(s)$ : mechanical system (including coupled 3D dynamics), cf. eqn. (A.51)

$S S C$ : sensor signal conditioning

$A D C$ : AD-converters (dSPACE DS2004) 
Table A.2: Model parameters of the electrical components

\begin{tabular}{|c|c|c|}
\hline Component & Parameter & Value \\
\hline \multirow{2}{*}{ ADCs } & number of bits & 16 \\
\hline & full scale range (bipolar) & $5 \mathrm{~V}$ \\
\hline DACs & number of bits & 16 \\
\hline DAC shaker & full scale range (bipolar) & $5 \mathrm{~V}$ \\
\hline DAC VCA & full scale range (bipolar) & $5 \mathrm{~V}$ \\
\hline DAC PEA & full scale range (bipolar) & $10 \mathrm{~V}$ \\
\hline \multirow{3}{*}{$\begin{array}{c}\text { Nexus } \\
\text { signal } \\
\text { conditioning }\end{array}$} & overall sensitivity $S_{o}$ & $3.16 \mathrm{~V} /\left(\mathrm{m} / \mathrm{s}^{2}\right)$ \\
\hline & high-pass cut-off ( $2^{\text {nd }}$ order $)$ & $0.1 \mathrm{~Hz}$ \\
\hline & low-pass cut-off ( $2^{\text {nd }}$ order $)$ & $1 \mathrm{kHz}$ \\
\hline \multirow{2}{*}{$\begin{array}{c}\text { piezo } \\
\text { amplifier }\end{array}$} & voltage gain $A_{v}$ & $6 \mathrm{~V} / \mathrm{V}$ \\
\hline & output impedance $R_{O}$ & $1 \mathrm{k} \Omega$ \\
\hline \multirow{2}{*}{$\begin{array}{c}\text { piezo } \\
\text { actuator }\end{array}$} & PE constant $e_{p}$ & $3.2 \mathrm{~N} / \mathrm{V}$ \\
\hline & (free) capacitance $C_{\mathrm{fr}}$ & $1600 \mathrm{nF}$ \\
\hline voice coil amplifier & voltage gain $A_{v}$ & $0.68 \mathrm{~V} / \mathrm{V}^{a}$ \\
\hline \multirow{3}{*}{$\begin{array}{c}\text { voice } \\
\text { coil } \\
\text { actuator }\end{array}$} & motor constant $k_{m}$ & $11.2 \mathrm{~N} / \mathrm{A}$ \\
\hline & coil resistance $R_{c}$ & $12.8 \Omega$ \\
\hline & coil inductance $L_{c}$ & $3 \mathrm{mH}$ \\
\hline shaker amplifier & voltage gain $A_{v}$ & $1 \mathrm{~V} / \mathrm{V}$ \\
\hline \multirow{2}{*}{ shaker } & motor constant $k_{m}$ & $6.4 \mathrm{~N} / \mathrm{A}$ \\
\hline & coil resistance $R_{c}$ & $2 \Omega$ \\
\hline
\end{tabular}

a Includes a 1/20 voltage divider circuit, nominally $13.6 \mathrm{~V} / \mathrm{V}$ 



\section{Appendix B}

\section{Feedback controller tuning for active damping}

In this appendix, several optimal tuning rules are derived for a direct velocity feedback (DVF) controller. The analysis is focussed on adding active damping to the structural mode. These results can also be found in [Preumont (2002); Vervoordeldonk et al. (2006)], albeit without the proof.

In section B.3, the relation between DVF and passivity-based control is discussed briefly.

\section{B.1 Model equation}

To find tuning rules for a DVF controller, an approximation for the loop gain in the vicinity of the structural resonance frequency $\omega_{r}$ is used. For this purpose, an approximation of the modal expansion of the system dynamics is used. This approximation can be expressed by the general form shown in equation (B.1). Here, $R^{m}$ and $R^{k}$ are the so-called mass and stiffness residues and $A$ is the contribution factor of the suspension resonance. The velocity feedback gain is denoted by $k_{v}$.

$$
L(s) \approx k_{v}\left(\frac{R^{m}}{s}+\frac{A s}{s^{2}+\omega_{r}^{2}}+R^{k} s\right)
$$

When the velocity measurement and the force actuation are collocated ${ }^{1}$, both residues and the contribution factor are positive. In case the mass residue term dominates over the stiffness residue, the loop gain can then be further

1 Measurement and actuation occur at the same location(s) 
approximated as shown in equation (B.2). On the other hand, when the stiffness residue term dominates, the approximation of equation (B.3) may be used.

$$
\begin{array}{ll}
L(s) \approx \frac{\bar{k}_{v 1}}{s} \frac{s^{2}+\omega_{a}^{2}}{s^{2}+\omega_{r}^{2}} & \bar{k}_{v 1}=k_{v}\left(A+R^{m}\right), \quad \omega_{a}^{2}=\frac{R^{m} \omega_{r}^{2}}{R^{m}+A}<\omega_{r}^{2} \\
L(s) \approx \bar{k}_{v 2} s \frac{s^{2}+\omega_{a}^{2}}{s^{2}+\omega_{r}^{2}} & \bar{k}_{v 2}=k_{v} R^{k}, \quad \omega_{a}^{2}=\omega_{r}^{2}+\frac{A}{R^{k}}>\omega_{r}^{2}
\end{array}
$$

\section{B.2 Maximum damping}

In this section, expressions are derived for the maximum achievable damping. The derivation is presented for the case of dominating mass residue (B.2), the derivation for dominating stiffness residue (B.3) follows a similar line.

The closed loop poles are the solutions to the characteristic equation (B.4). For the loop gain of equation (B.2), the resulting characteristic polynomial is then given by equation (B.5).

$$
\begin{aligned}
& 1+L(s)=0 \\
& s^{3}+\bar{k}_{v 1} s^{2}+\omega_{r}^{2} s+\bar{k}_{v 1} \omega_{a}^{2}=0
\end{aligned}
$$

Because equation (B.5) is a third order polynomial, it has at least one real root (at $s=-r)$. The other roots are assumed to be complex $\left(s=-\zeta \omega \pm j \omega \sqrt{1-\zeta^{2}}\right)$. Hence, the characteristic polynomial can be expressed as shown in equation (B.6).

$$
(s+r)\left(s^{2}+2 \zeta \omega s+\omega^{2}\right)=0
$$

By expanding equation (B.6) and matching coefficients with equation (B.5), an expression is found for $\zeta$ as a function of $\omega$, see equation (B.7).

$$
\zeta=\frac{\sqrt{\left(\omega_{r}^{2}-\omega^{2}\right)\left(\omega^{2}-\omega_{a}^{2}\right)}}{2 \omega \omega_{a}}
$$

By setting the derivative of $\zeta$ with respect to $\omega$ to zero, the maximum achievable damping can be found. The maximum damping, the required feedback gain and the corresponding frequency are listed in equation (B.8). The superscript $d$ refers to the fact that the parameters are optimized for maximum damping. 


$$
\left.\begin{array}{rl}
\omega_{\mathrm{opt}}^{\mathrm{d}} & =r_{\mathrm{opt}}^{\mathrm{d}}=\sqrt{\omega_{r} \omega_{a}} \\
\zeta_{\mathrm{opt}}^{\mathrm{d}} & =\frac{\omega_{r}-\omega_{a}}{2 \omega_{a}} \\
\bar{k}_{v 1, \mathrm{opt}}^{\mathrm{d}} & =\frac{\omega_{r}}{\omega_{a}} \sqrt{\omega_{a} \omega_{r}}
\end{array}\right\} \quad \text { for } \omega_{a}<\omega_{r}
$$

Following a similar line of reasoning for the loop gain of equation (B.3), it can be shown that the optimal result is then given by equation (B.9).

$$
\left.\begin{array}{rl}
\omega_{\mathrm{opt}}^{\mathrm{d}} & =r_{\mathrm{opt}}^{\mathrm{d}}=\sqrt{\omega_{r} \omega_{a}} \\
\zeta_{\mathrm{opt}}^{\mathrm{d}} & =\frac{\omega_{a}-\omega_{r}}{2 \omega_{r}} \\
\bar{k}_{v 2, \mathrm{opt}}^{\mathrm{d}} & =\frac{\omega_{a}}{\omega_{r} \sqrt{\omega_{a} \omega_{r}}}
\end{array}\right\} \quad \text { for } \omega_{r}<\omega_{a}
$$

\section{B.3 Passivity-based control}

Direct Velocity Feedback control using collocated actuator/sensor pairs is an example of passivity-based control, as will be explained here. In this appendix, only some basic results are presented for passive LTI systems, which are mainly adapted from the results for nonlinear systems in [Byrnes et al. (1991); Bao and Lee (2007)]. For a more in-depth discussion of passivitybased control, see e.g. [Ortega et al. (1998); van der Schaft (2000); Bao and Lee (2007)].

\section{B.3.1 Passivity}

Consider the state-space system of equation (B.10). Then, the system is passive when a continuous, positive semi-definite function $V(x)$ exists, such that $V(\mathbf{0})=0$ and the dissipation inequality (B.11) holds for all admissible inputs u. $V(\boldsymbol{x})$ is called a storage function.

$$
\begin{aligned}
& \dot{x}(t)=A \boldsymbol{x}(t)+\boldsymbol{B} \boldsymbol{u}(t) \quad \boldsymbol{x} \in \mathbb{R}^{n}, \boldsymbol{u}, \boldsymbol{y} \in \mathbb{R}^{m} \\
& y(t)=\boldsymbol{C} \boldsymbol{x}(t) \\
& V(\boldsymbol{x}(t))-V(\boldsymbol{x}(0)) \leq \int_{0}^{t} y^{\mathrm{T}}(\tau) \boldsymbol{u}(\tau) \mathrm{d} \tau, \quad \forall t \geq 0, \forall \boldsymbol{x}(0) \in \mathbb{R}^{n}
\end{aligned}
$$


When the storage function is continuously differentiable, the dissipation inequality (B.11) can be expressed by equation (B.12).

$$
\dot{V}(x(t)) \leq y^{\mathrm{T}}(t) \boldsymbol{u}(t), \quad \forall t \geq 0
$$

A linear passive system has the following properties (among others):

- the system is stable when $V(\boldsymbol{x})$ is continuously differentiable and positive definite ${ }^{2}$

- if the system is detectable ${ }^{3}$, (negative) static gain output feedback asymptotically stabilizes the system

- the system's transfer function $\boldsymbol{H}(s)$ is minimum phase

- for a SISO system, $\angle H(j \omega) \in\left[-90^{\circ}, 90^{\circ}\right] \forall \omega \in \mathbb{R}$

\section{B.3.2 DVF in terms of passivity-based control}

Consider the general lumped parameter model of a flexible structure in equation (B.13). Here, $\boldsymbol{q}$ is the vector of generalized coordinates, $\boldsymbol{d}$ and $\boldsymbol{u}$ are the (generalized) disturbances and actuator signals. $\boldsymbol{M}, \boldsymbol{D}$ and $\boldsymbol{K}$ are the (generalized) mass, damping and stiffness matrices. $\boldsymbol{M}$ is assumed to be positive definite, $\boldsymbol{D}$ and $\boldsymbol{K}$ are assumed to be positive semi-definite. As a result, the open loop system is (marginally) stable. The matrices $\boldsymbol{B}_{d}$ and $\boldsymbol{B}_{u}$ represent the interaction of the disturbances and actuators with the structure.

$$
M \ddot{q}+D \dot{q}+K q=B_{d} d+B_{u} u
$$

A state space representation in the form of equation (B.10) is easily found by choosing the state vector $\boldsymbol{x}=\left[\begin{array}{ll}\boldsymbol{q}^{\mathrm{T}} & \dot{\boldsymbol{q}}^{\mathrm{T}}\end{array}\right]^{\mathrm{T}}$. Then, a positive definite candidate storage function is the total system energy, see equation (B.14). Note that the only equilibrium is then given by $(\boldsymbol{q}, \dot{\boldsymbol{q}})=(\mathbf{0}, \mathbf{0})$. The time derivative of $V(\boldsymbol{q}, \dot{\boldsymbol{q}})$ is given by equation (B.15).

$$
\begin{gathered}
V(\boldsymbol{x})=V(\boldsymbol{q}, \dot{\boldsymbol{q}})=\frac{1}{2} \dot{\boldsymbol{q}}^{\mathrm{T}} \boldsymbol{M} \dot{\boldsymbol{q}}+\frac{1}{2} \boldsymbol{q}^{\mathrm{T}} \boldsymbol{K} \boldsymbol{q}>0 \quad \forall(\boldsymbol{q}, \dot{\boldsymbol{q}}) \neq(\mathbf{0}, \mathbf{0}) \\
\dot{V}(\boldsymbol{q}, \dot{\boldsymbol{q}})=\dot{\boldsymbol{q}}^{\mathrm{T}}(\boldsymbol{M} \ddot{\boldsymbol{q}}+\boldsymbol{K} \boldsymbol{q})=\dot{\boldsymbol{q}}^{\mathrm{T}}\left(\boldsymbol{B}_{d} \boldsymbol{d}+\boldsymbol{B}_{u} \boldsymbol{u}-\boldsymbol{D} \dot{\boldsymbol{q}}\right)
\end{gathered}
$$

Now, we try to find a feedback control law and an output measurement that render the closed loop system asymptotically stable.

2 In this case, $V(x)$ is a Lyapunov function (evaluate equation (B.12) for $\boldsymbol{u} \equiv 0$ )

3 The system is detectable when all unstable modes are observable 
In order to assess closed loop stability, we are interested in the unforced response, i.e. the disturbance inputs can be ignored $(\boldsymbol{d} \equiv 0)$. Moreover, because $D \geq 0$ by assumption, the inequality in equation (B.16) holds.

$$
\left.\dot{V}(\boldsymbol{q}, \dot{\boldsymbol{q}})\right|_{\boldsymbol{d} \equiv 0}=\dot{\boldsymbol{q}}^{\mathrm{T}}\left(\boldsymbol{B}_{u} \boldsymbol{u}-\boldsymbol{D} \dot{\boldsymbol{q}}\right) \leq \dot{\boldsymbol{q}}^{\mathrm{T}} \boldsymbol{B}_{u} \boldsymbol{u}
$$

By choosing collocated velocity measurement according to equation (B.17), the dissipation inequality (B.12) is satisfied. Therefore, the open loop system is passive.

Then, the second property listed in section B.3.1 states that the static gain output feedback of equation (B.18) asymptotically stabilizes the closed loop system, provided that any undetectable modes are stable ${ }^{4}$. Indeed, from equation (B.19), we can conclude that $V(\boldsymbol{q}, \dot{\boldsymbol{q}})$ is a Lyapunov function for the closed loop system with a negative definite time-derivative. Therefore, the closed loop system is asymptotically stable.

$$
\begin{aligned}
y & =\boldsymbol{B}_{u}^{\mathrm{T}} \dot{\boldsymbol{q}} \\
\boldsymbol{u} & =-\boldsymbol{G} y, \quad \boldsymbol{G}>0 \\
\dot{V}(\boldsymbol{q}, \dot{\boldsymbol{q}}) & \leq \dot{\boldsymbol{q}}^{\mathrm{T}} \boldsymbol{B}_{u} \boldsymbol{u}=-\boldsymbol{y}^{\mathrm{T}} \boldsymbol{G} \boldsymbol{y}<0 \quad \forall y \neq \mathbf{0}
\end{aligned}
$$

4 Detectable modes are also stabilizable, due to the collocated measurement 



\section{Appendix C}

\section{Causal Wiener filter}

In this appendix, an outline of the proof for the Causal Wiener filter is presented, which is based on [Fraanje (2004), Appendix A].

The Causal Wiener filter is the solution to the minimization problem (C.2), where $\boldsymbol{W}(z)$ is constrained to be causal and stable. See sections 5.1 and 5.2 for the definition of the dynamic systems.

$$
\begin{aligned}
J_{\mathrm{MSE}}(\boldsymbol{W}) & =\left\|\boldsymbol{P}_{\boldsymbol{d}}(z)+\boldsymbol{S}(z) \boldsymbol{W}(z) \boldsymbol{T}_{\boldsymbol{d}}(z)\right\|_{2}^{2} \\
\boldsymbol{W}_{c}(z) & =\arg \min _{\boldsymbol{W}} J_{\mathrm{MSE}}(\boldsymbol{W})
\end{aligned}
$$

For the derivation of the Causal Wiener filter, the inner-outer and co-innerouter factorization are used, which are defined in section C.1 (see also section 5.2.2). The derivation of the Causal Wiener filter is presented in section C.2.

\section{C.1 (Co-) inner-outer factorization}

The inner-outer factorization of the stable, proper system $G(z)$ is given by equation (C.3). $\boldsymbol{G}(z)$ has $N_{u}$ inputs and $N_{y}$ outputs and normal rank $R=$ $\sup \{\operatorname{rank}(\boldsymbol{G}(\lambda)), \lambda \in \mathbb{C}, \lambda$ not a pole of $G(z)\}$. For well-defined systems, the normal rank $R$ equals $\min \left(N_{y}, N_{u}\right)$. However, for example, a $3 \times 2$ system whose inputs are linearly dependent has a normal rank of only 1.

$$
G(z)=G_{i}(z) G_{o}(z)
$$


The outer factor $G_{o}(z)$ is stable, proper and minimum-phase. It has $N_{u}$ inputs and $R$ outputs. Because it is minimum-phase and $R \leq N_{u}$, it has (at least) a stable right inverse. The inner factor $\boldsymbol{G}_{i}(z)$ is stable, proper and all-pass. The inner factor has $R$ inputs and $N_{y}$ outputs. When the inner factor is not square $\left(R<N_{y}\right)$, an orthogonal inner factor $G_{i}^{\perp}(z)$ exists with $N_{y}$ outputs and $N_{y}-R$ inputs, such that $\left[G_{i}(z) G_{i}^{\perp}(z)\right]$ is a unitary system ${ }^{1}$, see equations (C.4) and (C.5). Here, $G_{i}^{*}(z)$ denotes the adjoint system of $G_{i}(z)$, which denotes the transposed and time-reversed system: $G_{i}^{*}(z) \equiv G_{i}^{\mathrm{T}}\left(z^{-1}\right)$.

$$
\begin{aligned}
& \boldsymbol{G}_{i}(z) \boldsymbol{G}_{i}(z)^{*}+\boldsymbol{G}_{i}^{\perp}(z) \boldsymbol{G}_{i}^{\perp *}(z)=\boldsymbol{I}_{N_{y}} \\
& {\left[\begin{array}{cc}
\boldsymbol{G}_{i}^{*}(z) \boldsymbol{G}_{i}(z) & \boldsymbol{G}_{i}^{*}(z) \boldsymbol{G}_{i}^{\perp}(z) \\
\boldsymbol{G}_{i}^{\perp *}(z) \boldsymbol{G}_{i}(z) & \boldsymbol{G}_{i}^{\perp *}(z) \boldsymbol{G}_{i}^{\perp}(z)
\end{array}\right]=\left[\begin{array}{cc}
\boldsymbol{I}_{R} & \mathbf{0} \\
\mathbf{0} & \boldsymbol{I}_{N_{y}-R}
\end{array}\right]}
\end{aligned}
$$

The co-inner-outer factorization is closely related to the inner-outer factorization. Given the inner-outer factorization (C.3) of $G(z)$, the co-inner-outer factorization of $\boldsymbol{H}(z) \equiv \boldsymbol{G}^{\mathrm{T}}(z)$ is given by equation (C.6). In words, the co-inner and co-outer factors of $\boldsymbol{H}(z)$ are the transposed inner and outer factors of the transpose of $\boldsymbol{H}(z)$.

$$
\boldsymbol{H}(z)=\boldsymbol{H}_{c o}(z) \boldsymbol{H}_{c i}(z)=\boldsymbol{G}_{o}^{\mathrm{T}}(z) \boldsymbol{G}_{i}^{\mathrm{T}}(z)
$$

Once again, if the co-inner factor $\boldsymbol{H}_{c i}(z)$ is not square, an orthogonal co-inner factor $\boldsymbol{H}_{c i}^{\perp}(z)$ exists, such that $\left[\boldsymbol{H}_{c i}^{*}(z) \boldsymbol{H}_{c i}^{\perp *}(z)\right]$ is unitary. This means that equations (C.4) and (C.5) are also valid when $G_{i}(z)$ and $G_{i}^{\perp}(z)$ are replaced by $\boldsymbol{H}_{c i}^{*}(z)$ and $\boldsymbol{H}_{c i}^{\perp *}(z)$, see equations (C.7) and (C.8). Here, $\boldsymbol{H}(z)$ has $N_{y}$ outputs, $N_{u}$ inputs and normal rank $R$.

$$
\begin{aligned}
& \boldsymbol{H}_{c i}(z)^{*} \boldsymbol{H}_{c i}(z)+\boldsymbol{H}_{c i}^{\perp *}(z) \boldsymbol{H}_{c i}^{\perp}(z)=\boldsymbol{I}_{N_{u}} \\
& {\left[\begin{array}{ll}
\boldsymbol{H}_{c i}(z) \boldsymbol{H}_{c i}^{*}(z) & \boldsymbol{H}_{c i}(z) \boldsymbol{H}_{c i}^{\perp *}(z) \\
\boldsymbol{H}_{c i}^{\perp}(z) \boldsymbol{H}_{c i}^{*}(z) & \boldsymbol{H}_{c i}^{\perp}(z) \boldsymbol{H}_{c i}^{\perp *}(z)
\end{array}\right]=\left[\begin{array}{cc}
\boldsymbol{I}_{R} & \mathbf{0} \\
\mathbf{0} & \boldsymbol{I}_{N_{u}-R}
\end{array}\right]}
\end{aligned}
$$

Note that, for SISO systems, the inner-outer and co-inner-outer factorization coincide. Moreover, the orthogonal (co-)inner factors do not exist for SISO systems.

See section C.3 for a state-space algorithm to compute the (co-)inner-outer factorization.

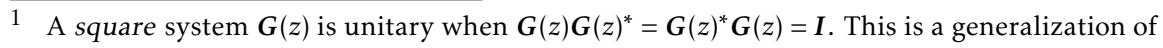
the concept of unitary matrices to linear systems. 


\section{C.2 Derivation of the Causal Wiener filter}

Using property (C.4) of the inner factor of $S(z)$ and the equivalent property (C.7) for the co-inner factor of $\boldsymbol{T}_{\boldsymbol{d}}(z)$, the system $\boldsymbol{P}_{\boldsymbol{d}}(z)$ can be expressed by equation (C.9). The dependency on $z$ has been dropped, for ease of notation.

$$
\begin{aligned}
\boldsymbol{P}_{\boldsymbol{d}}= & \left(S_{i} S_{i}^{*}+S_{i}^{\perp} S_{i}^{\perp *}\right) \boldsymbol{P}_{\boldsymbol{d}}\left(\boldsymbol{T}_{\boldsymbol{d}, c i}^{*} \boldsymbol{T}_{\boldsymbol{d}, c i}+\boldsymbol{T}_{\boldsymbol{d}, c i}^{\perp *} \boldsymbol{T}_{\boldsymbol{d}, c i}^{\perp}\right) \\
= & S_{i} S_{i}^{*} \boldsymbol{P}_{\boldsymbol{d}} \boldsymbol{T}_{\boldsymbol{d}, c i}^{*} \boldsymbol{T}_{c i}+S_{i} S_{i}^{*} \boldsymbol{P}_{\boldsymbol{d}} \boldsymbol{T}_{\boldsymbol{d}, c i}^{\perp *} \boldsymbol{T}_{\boldsymbol{d}, c i}^{\perp} \\
& \quad+S_{i}^{\perp} S_{i}^{\perp *} \boldsymbol{P}_{\boldsymbol{d}} \boldsymbol{T}_{\boldsymbol{d}, c i}^{*} \boldsymbol{T}_{\boldsymbol{d}, c i}+S_{i}^{\perp} S_{i}^{\perp *} \boldsymbol{P}_{\boldsymbol{d}} \boldsymbol{T}_{\boldsymbol{d}, c i}^{\perp *} \boldsymbol{T}_{\boldsymbol{d}, c i}^{\perp} \\
\equiv & \boldsymbol{P}_{1}+\boldsymbol{P}_{2}+\boldsymbol{P}_{3}+\boldsymbol{P}_{4}
\end{aligned}
$$

Note that, due to the property (C.5) of the inner factor of $S(z)$ and the equivalent property (C.8) for the co-inner factor of $\boldsymbol{T}_{\boldsymbol{d}}(z)$, the four terms $\boldsymbol{P}_{1}, \boldsymbol{P}_{2}, \boldsymbol{P}_{3}$ and $\boldsymbol{P}_{4}$ are orthogonal (in the sense of the inner product), see equation (C.10).

$$
\left\langle\boldsymbol{P}_{k}, \boldsymbol{P}_{l}\right\rangle \equiv \frac{1}{2 \pi} \operatorname{tr} \int_{-\pi}^{\pi} \boldsymbol{P}_{k}\left(\mathrm{e}^{j \omega}\right) \boldsymbol{P}_{l}\left(\mathrm{e}^{j \omega}\right)^{\mathrm{H}} \mathrm{d} \omega=0, \quad k \neq l \in\{1,2,3,4\}
$$

The second term in the optimization criterion (C.1) can be expressed by equation (C.11). Note that this term is also orthogonal to $\boldsymbol{P}_{2}, \boldsymbol{P}_{3}$ and $\boldsymbol{P}_{4}$.

$$
S W T_{d}=S_{i} S_{o} W T_{d, c o} T_{d, c i}
$$

It is well known that the squared 2-norm of the sum of two orthogonal systems $\boldsymbol{G}, \boldsymbol{H}$ is just the sum of the squared 2-norms of the individual systems, see equation (C.12). Then, the cost criterion (C.1) can be expressed by equation (C.13), with $W$ constrained to be stable and causal.

$$
\begin{aligned}
& \|\boldsymbol{G}+\boldsymbol{H}\|_{2}^{2}=\|\boldsymbol{G}\|_{2}^{2}+\|\boldsymbol{H}\|_{2}^{2} \Leftrightarrow\langle\boldsymbol{G}, \boldsymbol{H}\rangle=0 \\
& J(\boldsymbol{W})=\left\|\boldsymbol{P}_{1}+S \boldsymbol{W} \boldsymbol{T}_{\boldsymbol{d}}\right\|_{2}^{2}+\left\|\boldsymbol{P}_{2}\right\|_{2}^{2}+\left\|\boldsymbol{P}_{3}\right\|_{2}^{2}+\left\|\boldsymbol{P}_{4}\right\|_{2}^{2}
\end{aligned}
$$

Only the first term in equation (C.13) depends on $\boldsymbol{W}$. Therefore, the Causal Wiener filter is also the solution to the minimization problem (C.14). Moreover, the alternative cost criterion $\tilde{J}(\boldsymbol{W})$ can be reduced to equation (C.15), due to the all-pass properties of $S_{i}$ and $T_{d, c i}$.

$$
\begin{aligned}
& \boldsymbol{W}_{c}=\arg \min _{\boldsymbol{W}} \tilde{J}(\boldsymbol{W})=\arg \min _{\boldsymbol{W}}\left\|\boldsymbol{P}_{1}+\boldsymbol{S} \boldsymbol{W} \boldsymbol{T}_{\boldsymbol{d}}\right\|_{2}^{2} \\
& \tilde{J}(\boldsymbol{W})=\left\|\boldsymbol{P}_{1}+\boldsymbol{S} \boldsymbol{W} \boldsymbol{T}_{\boldsymbol{d}}\right\|_{2}^{2}=\left\|\boldsymbol{S}_{i}^{*} \boldsymbol{P}_{\boldsymbol{d}} \boldsymbol{T}_{\boldsymbol{d}, c i}^{*}+\boldsymbol{S}_{o} \boldsymbol{W} \boldsymbol{T}_{\boldsymbol{d}, c o}\right\|_{2}^{2}
\end{aligned}
$$


The first term $S_{i}^{*} \boldsymbol{P}_{\boldsymbol{d}} \boldsymbol{T}_{\boldsymbol{d}, c i}^{*}$ in equation (C.15) is stable, but may be acausal ${ }^{2}$ due to the presence of the adjoint systems. However, because $W$ is constrained to be stable and causal, the second term $\boldsymbol{S}_{o} \boldsymbol{W} \boldsymbol{T}_{\boldsymbol{d} \text {,co }}$ is stable and causal. Therefore, the anti-causal part of the first term can not be compensated for by the Causal Wiener filter.

Mathematically, this can be shown as outlined below. For any two stable systems $\boldsymbol{X}, \boldsymbol{Y}$ of equal dimensions, equation (C.16) holds. Here, $\left\{\bullet_{+}\right.$and $\{\bullet\}_{-}$ denote the causal and anti-causal parts of $\bullet$ respectively. See section C.3 for an implementation of the (anti-)causality operators.

The proof follows from the definition of the $\mathcal{H}_{2}$-norm ${ }^{3}$ and the orthogonality property (C.17), which is easily obtained from the expansion of each system into powers of $z$ and evaluating the inner product.

$$
\begin{aligned}
& \left\|\{\boldsymbol{X}\}_{+}+\{\boldsymbol{Y}\}_{-}\right\|_{2}^{2}=\left\|\{\boldsymbol{X}\}_{+}\right\|_{2}^{2}+\left\|\{\boldsymbol{Y}\}_{-}\right\|_{2}^{2} \\
& \left\langle\{\boldsymbol{X}\}_{+},\{\boldsymbol{Y}\}_{-}\right\rangle=0
\end{aligned}
$$

Therefore, the cost criterion can be expressed by equation (C.18). Moreover, because $S_{o}$ has a stable right inverse $S_{o}^{\ddagger}$ and $T_{\boldsymbol{d}, c o}$ has a stable left inverse $T_{d, c o}^{\dagger}$, it is immediately clear that the cost criterion is minimized by the Causal Wiener filter $\boldsymbol{W}_{c}$, as given by equation (C.19).

$$
\begin{aligned}
& \tilde{J}(W)=\left\|\left\{S_{i}^{*} \boldsymbol{P}_{\boldsymbol{d}} \boldsymbol{T}_{d, c i}^{*}\right\}_{-}\right\|_{2}^{2}+\left\|\left\{S_{i}^{*} \boldsymbol{P}_{\boldsymbol{d}} \boldsymbol{T}_{d, c i}^{*}\right\}_{+}+S_{o} W \boldsymbol{T}_{\boldsymbol{d}, c o}\right\|_{2}^{2} \\
& \boldsymbol{W}_{c}=-S_{o}^{\ddagger}\left\{S_{i}^{*} \boldsymbol{P}_{\boldsymbol{d}} \boldsymbol{T}_{d, c i}^{*}\right\}_{+} \boldsymbol{T}_{c o}^{+}
\end{aligned}
$$

It can be seen that the Causal Wiener filter uses the outer factor $S_{o}$ and the co-outer factor $\boldsymbol{T}_{\boldsymbol{d}, c o}$ to causally invert as much of the dynamics of $S$ and $\boldsymbol{T}_{\boldsymbol{d}}$ as possible. The remaining dynamics of these paths can only be compensated by the anti-causal adjoint systems of the (co-)inner factors. To obtain a causal controller, the causal part of these terms combined with $\boldsymbol{P}_{\boldsymbol{d}}$ is taken.

The minimum value of the MSE cost criterion $J(W)$ is then given by equation (C.20). Here, the last two terms represent an equivalent expression for $\left\|\boldsymbol{P}_{2}\right\|_{2}^{2}+$ $\left\|\boldsymbol{P}_{3}\right\|_{2}^{2}+\left\|\boldsymbol{P}_{4}\right\|_{2}^{2}$ in equation (C.13).

$$
J\left(\boldsymbol{W}_{c}\right)=\left\|\left\{S_{i}^{*} \boldsymbol{P}_{\boldsymbol{d}} \boldsymbol{T}_{\boldsymbol{d}, c i}^{*}\right\}_{-}\right\|_{2}^{2}+\left\|\boldsymbol{S}_{i}^{\perp *} \boldsymbol{P}_{\boldsymbol{d}}\right\|_{2}^{2}+\left\|\boldsymbol{S}_{i}^{*} \boldsymbol{P}_{\boldsymbol{d}} \boldsymbol{T}_{\boldsymbol{d}, c i}^{\perp *}\right\|_{2}^{2}
$$

2 A system is causal if it depends on present and past inputs only. It is anti-causal when it depends on future inputs only. It is called acausal when it depends on future and past and/or present inputs.

3 See section 2.3 for the continuous time counterpart, the discrete time definition is defined equivalently. 
The three terms in equation (C.20) can be given the following interpretation: the first term is due to non-minimum phase zeros and delays in $S$ and $T_{d}$ which are not cancelled by unstable poles and delays in $\boldsymbol{P}_{\boldsymbol{d}}$.

The second term only exists when $S_{i}^{\perp}$ exists. For vibration isolation problems, this occurs when there are more error signals than actuator signals. In this case it is clear that, unless $\boldsymbol{S}_{i}^{\perp}$ and $\boldsymbol{P}_{\boldsymbol{d}}$ are orthogonal, perfect cancellation is not achievable, even if there are no delays and non-minimum phase zeros in $\boldsymbol{S}$. The mentioned orthogonality of $\boldsymbol{S}_{i}^{\perp}$ and $\boldsymbol{P}_{\boldsymbol{d}}$ occurs when the disturbance sources only cause vibrations that can be controlled by the actuators, which is very unlikely to be the case in any practical situation.

Similarly, the third term only exists when $T_{\boldsymbol{d}, c i}^{\perp}$ exists. For vibration isolation problems, this occurs when there are more disturbance sources than reference sensors, i.e. it is not possible to independently determine the contribution of all disturbance sources to the reference signals. Unless $\boldsymbol{P}_{\boldsymbol{d}}$ and $\boldsymbol{T}_{\boldsymbol{d}, c i}^{\perp}$ are orthogonal, perfect cancellation can not be achieved. This orthogonality occurs only when the (combinations of) disturbance sources that are not measurable at the reference sensors do not cause any vibration at the error sensors. Again, this is highly unlikely to occur in practice.

\section{C.3 Implementation of the (co-)inner-outer factor- ization and the (anti-) causality operator}

In this section, state-space formulae for the (co-)inner-outer factorization and the (anti-)causality operator are given. These formulae are taken from [Ionescu and Oară (1996); Fraanje (2004), Appendix B].

\section{Implementation of the (co-)inner-outer factorization}

Let the discrete system $G(z)$ be given by the state-space representation (C.21).

$$
\begin{aligned}
\boldsymbol{x}(k+1) & =\boldsymbol{A} \boldsymbol{x}(k)+\boldsymbol{B} \boldsymbol{u}(k) \\
y(k) & =\boldsymbol{C} \boldsymbol{x}(k)+\boldsymbol{D} \boldsymbol{u}(k)
\end{aligned} \quad \boldsymbol{x} \in \mathbb{R}^{N_{x}}, \boldsymbol{u} \in \mathbb{R}^{N_{u}}, y \in \mathbb{R}^{N_{y}}
$$

If $G(z)$ is stable, without zeros on the unit circle, and $N_{y} \geq N_{u}$, the inner factor $\boldsymbol{G}_{i}(z)$ and outer factor $\boldsymbol{G}_{o}(z)$ of $\boldsymbol{G}(z)$ are given by equation (C.22).

$$
G_{i}(z) \sim\left[\begin{array}{cc}
A-B F & B H^{\ddagger} \\
C-D F & D H^{\ddagger}
\end{array}\right], \quad G_{o}(z) \sim\left[\begin{array}{cc}
A & B \\
H F & H
\end{array}\right]
$$


Here, $\bullet$ denotes the right inverse and $\boldsymbol{X}=\boldsymbol{X}^{\mathrm{T}}$ is the unique, stabilizing solution to the discrete algebraic Riccati equation (DARE) (C.23). $\boldsymbol{H}$ satisfies equation (C.24) and can be computed by a Cholesky decomposition. Finally, $\boldsymbol{F}$ is given by equation (C.25). The DARE and Cholesky decomposition can be performed by standard MATLAB routines.

$$
\begin{aligned}
& \boldsymbol{X}=\boldsymbol{A}^{\mathrm{T}} \boldsymbol{X} \boldsymbol{A}+\boldsymbol{C}^{\mathrm{T}} \boldsymbol{C} \\
& \quad-\left(\boldsymbol{A}^{\mathrm{T}} \boldsymbol{X} \boldsymbol{B}+\boldsymbol{C}^{\mathrm{T}} \boldsymbol{D}\right)\left(\boldsymbol{D}^{\mathrm{T}} \boldsymbol{D}+\boldsymbol{B}^{\mathrm{T}} \boldsymbol{X} \boldsymbol{B}\right)^{-1}\left(\boldsymbol{D}^{\mathrm{T}} \boldsymbol{C}+\boldsymbol{B}^{\mathrm{T}} \boldsymbol{X} \boldsymbol{A}\right) \\
& \boldsymbol{H}^{\mathrm{T}} \boldsymbol{H}=\boldsymbol{D}^{\mathrm{T}} \boldsymbol{D}+\boldsymbol{B}^{\mathrm{T}} \boldsymbol{X} \boldsymbol{B} \\
& \boldsymbol{F}=\left(\boldsymbol{D}^{\mathrm{T}} \boldsymbol{D}+\boldsymbol{B}^{\mathrm{T}} \boldsymbol{X} \boldsymbol{B}\right)^{-1}\left(\boldsymbol{D}^{\mathrm{T}} \boldsymbol{C}+\boldsymbol{B}^{\mathrm{T}} \boldsymbol{X} \boldsymbol{A}\right)
\end{aligned}
$$

Note that these formulae are only valid when $N_{y} \geq N_{u}$, although this covers most practical situations. The requirement that $\boldsymbol{G}(z)$ has no zeros on the unit circle can be enforced (if not satisfied naturally) by augmenting the system as shown in equation (C.26), where $\rho$ is a small positive number.

$$
\boldsymbol{G}_{\mathrm{aug}}(z)=\left[\begin{array}{c}
\boldsymbol{G}(z) \\
\rho
\end{array}\right]
$$

As expressed in equation (C.6), the co-inner-outer factorization of a system $G(z)$ returns the transposed inner and outer factors of the transposed system $G^{\mathrm{T}}(z)$. Therefore, the same formulae can be used. Note that, for the coinner-outer factorization to be computable by the formulae presented here, the number of inputs must now be larger than or equal to the number of outputs $\left(N_{u} \geq N_{y}\right)$.

Finally, it is pointed out that for discrete SISO systems in transfer function or zero-pole-gain representation the (co-)inner-outer factorization can be computed by grouping the unstable zeros in the inner factor and replacing these unstable zeros in the outer factor with their mirrored (stable) counterparts. These mirrored zeros are also the poles of the inner factor. Furthermore, a gain correction may be required.

As an example, consider the SISO system $G(z)$ of equation (C.27). Clearly this system is stable, but has an unstable zero at $z=2$. It is straightforward to show that the inner-outer factorization of $G(z)$ is given by equation C. 28 .

$$
\begin{aligned}
& G(z)=\frac{z-2}{z-0.8} \\
& G_{i}(z)=\frac{z-2}{2(z-0.5)}, \quad G_{o}(z)=\frac{2(z-0.5)}{z-0.8}
\end{aligned}
$$




\section{Implementation of the (anti-)causality operator}

For the computation of the Causal Wiener filter and the resulting cost criterion, the causal and anti-causal parts of a system $\boldsymbol{H}(\boldsymbol{z})$ of the form (C.29) are needed.

It is straightforward to show that the causal part $\boldsymbol{H}_{c}(z)$ and the anti-causal part $\boldsymbol{H}_{a c}(\boldsymbol{z})$ are then given by equation (C.30), where the dependency on $z$ has been dropped for ease of notation.

$$
\begin{aligned}
\boldsymbol{H}(z) & =\boldsymbol{H}_{1}^{*}(z) \boldsymbol{H}_{2}(z) \boldsymbol{H}_{3}^{*}(z) \\
\boldsymbol{H} & =\left\{\boldsymbol{H}_{1}^{*} \boldsymbol{H}_{2}\right\}_{+} \boldsymbol{H}_{3}^{*}+\left\{\boldsymbol{H}_{1}^{*} \boldsymbol{H}_{2}\right\}_{-} \boldsymbol{H}_{3}^{*} \\
= & \underbrace{\left\{\left\{\boldsymbol{H}_{1}^{*} \boldsymbol{H}_{2}\right\}_{+} \boldsymbol{H}_{3}^{*}\right\}_{+}}_{\boldsymbol{H}_{c}}+\underbrace{\left\{\left\{\boldsymbol{H}_{1}^{*} \boldsymbol{H}_{2}\right\}_{+} \boldsymbol{H}_{3}^{*}\right\}_{-}+\left\{\boldsymbol{H}_{1}^{*} \boldsymbol{H}_{2}\right\}_{-} \boldsymbol{H}_{3}^{*}}_{\boldsymbol{H}_{a c}}
\end{aligned}
$$

Therefore, the causal part of $\boldsymbol{H}(z)$ is computed in two stages:

1. compute the causal part $\boldsymbol{H}_{4}$ of $\boldsymbol{H}_{1}^{*} \boldsymbol{H}_{2}$

2. compute the causal part of $\boldsymbol{H}_{4} \boldsymbol{H}_{3}^{*}$

Assume that $\boldsymbol{H}_{1}(z), \boldsymbol{H}_{2}(z)$ and $\boldsymbol{H}_{3}(z)$ have state-space representations as given by equation (C.31).

$$
\boldsymbol{H}_{1}(z) \sim\left[\begin{array}{ll}
A_{1} & B_{1} \\
C_{1} & D_{1}
\end{array}\right], \quad H_{2}(z) \sim\left[\begin{array}{ll}
A_{2} & B_{2} \\
C_{2} & D_{2}
\end{array}\right], \quad H_{3}(z) \sim\left[\begin{array}{ll}
A_{3} & B_{3} \\
C_{3} & D_{3}
\end{array}\right]
$$

Then, the causal part of $\boldsymbol{H}_{1}^{*}(z) \boldsymbol{H}_{2}(z)$ is given by equation (C.32). Here, $\boldsymbol{X}_{12}$ is the solution to the discrete-time Sylvester equation (C.33).

$$
\begin{aligned}
& \boldsymbol{H}_{4}(z) \sim\left[\begin{array}{cc}
\boldsymbol{A}_{4} & \boldsymbol{B}_{4} \\
\boldsymbol{C}_{4} & \boldsymbol{D}_{4}
\end{array}\right]=\left[\begin{array}{cc}
\boldsymbol{A}_{2} & \boldsymbol{B}_{2} \\
\boldsymbol{D}_{1}^{\mathrm{T}} \boldsymbol{C}_{2}+\boldsymbol{B}_{1}^{\mathrm{T}} \boldsymbol{X}_{12} \boldsymbol{A}_{2} & \boldsymbol{D}_{1}^{\mathrm{T}} \boldsymbol{D}_{2}+\boldsymbol{B}_{1}^{\mathrm{T}} \boldsymbol{X}_{12} \boldsymbol{B}_{2}
\end{array}\right] \\
& \boldsymbol{A}_{1}^{\mathrm{T}} \boldsymbol{X}_{12} \boldsymbol{A}_{2}-\boldsymbol{X}_{12}+\boldsymbol{C}_{1}^{\mathrm{T}} \boldsymbol{C}_{2}=\mathbf{0}
\end{aligned}
$$

Using this result, the overall causal part $\boldsymbol{H}_{c}(z)$ is given by equation (C.34). Here, $\boldsymbol{X}_{43}$ is the solution to another discrete-time Sylvester equation (C.35).

$$
\begin{aligned}
& \boldsymbol{H}_{c}(z) \sim\left[\begin{array}{ll}
\boldsymbol{A}_{4} & \boldsymbol{B}_{4} \boldsymbol{D}_{3}^{\mathrm{T}}+\boldsymbol{A}_{4} \boldsymbol{X}_{43} \boldsymbol{C}_{3}^{\mathrm{T}} \\
\boldsymbol{C}_{4} & \boldsymbol{D}_{4} \boldsymbol{D}_{3}^{\mathrm{T}}+\boldsymbol{C}_{4} \boldsymbol{X}_{43} \boldsymbol{C}_{3}^{\mathrm{T}}
\end{array}\right] \\
& \boldsymbol{A}_{4} \boldsymbol{X}_{43} \boldsymbol{A}_{3}^{\mathrm{T}}-\boldsymbol{X}_{43}+\boldsymbol{B}_{4} \boldsymbol{B}_{3}^{\mathrm{T}}=\mathbf{0}
\end{aligned}
$$


The accompanying anti-causal parts require a so-called descriptor state-space representation, see equation (C.36). For anti-causal systems, the matrix $\boldsymbol{E}$ is singular. Equation (C.37) defines a shorthand notation for a descriptor statespace system $G(z)$.

$$
\begin{aligned}
\boldsymbol{E x}(k+1) & =\boldsymbol{A} \boldsymbol{x}(k)+\boldsymbol{B} \boldsymbol{u}(k) \\
y(k) & =\boldsymbol{C} \boldsymbol{x}(k)+\boldsymbol{D} \boldsymbol{u}(k)
\end{aligned} \quad \boldsymbol{x} \in \mathbb{R}^{N_{x}}, \boldsymbol{u} \in \mathbb{R}^{N_{u}}, \boldsymbol{y} \in \mathbb{R}^{N_{y}}
$$

Using this shorthand notation, the anti-causal part of $\boldsymbol{H}_{1}^{*}(z) \boldsymbol{H}_{2}(z)$ and the anticausal part of $\boldsymbol{H}_{4}(z) \boldsymbol{H}_{3}^{*}(z)$ are given by equations (C.38) and (C.39), respectively. The total anti-causal part $\boldsymbol{H}_{a c}(z)$ then follows from equation (C.30).

$$
\begin{aligned}
& \left\{\boldsymbol{H}_{1}^{*}(z) \boldsymbol{H}_{2}(z)\right\}_{-} \sim\left[\begin{array}{cc|cc|c}
\boldsymbol{A}_{1}^{\mathrm{T}} & \boldsymbol{C}_{1}^{\mathrm{T}} \boldsymbol{D}_{2}+\boldsymbol{A}_{1}^{\mathrm{T}} \boldsymbol{X}_{12} \boldsymbol{B}_{2} & \boldsymbol{I} & \mathbf{0} & \mathbf{0} \\
\mathbf{0} & \mathbf{0} & \mathbf{0} & \boldsymbol{I} & -\boldsymbol{I} \\
\hline \mathbf{0} & \mathbf{0} & \boldsymbol{B}_{1}^{\mathrm{T}} & \mathbf{0} & \mathbf{0}
\end{array}\right] \\
& \left\{\boldsymbol{H}_{4}(z) \boldsymbol{H}_{3}^{*}(z)\right\}_{-} \sim\left[\begin{array}{cc|cc|c}
\boldsymbol{A}_{3}^{\mathrm{T}} & \boldsymbol{C}_{3}^{\mathrm{T}} & \boldsymbol{I} & \mathbf{0} & \mathbf{0} \\
\mathbf{0} & \mathbf{0} & \mathbf{0} & \boldsymbol{I} & -\boldsymbol{I} \\
\hline \mathbf{0} & \mathbf{0} & \boldsymbol{D}_{4} \boldsymbol{B}_{3}^{\mathrm{T}}+\boldsymbol{C}_{4} \boldsymbol{X}_{43} \boldsymbol{A}_{3}^{\mathrm{T}} & \mathbf{0} & \mathbf{0}
\end{array}\right]
\end{aligned}
$$

The discrete-time Sylvester equations (C.33) and (C.35) can be solved using MATLAB's dlyap command. Moreover, the descriptor state-space systems (C.38) and (C.39) can be formed using the dss command.

Finally, it is pointed out that for discrete SISO systems in transfer function or zero-pole-gain representation the anti-causal and causal parts can be computed by partial fraction expansion. 


\section{Appendix D}

\section{Geophone noise analysis}

In this appendix, the noise analysis is described for the geophone and its associated amplifier, as discussed in section 6.1.5. The analysis is based on [Texas Instruments (2007)]. Similar approaches have been applied for geophone circuitry in [Riedesel et al. (1990); Barzilai et al. (1998)].

\section{D.1 Geophone model and signal conditioning}

The operation of a geophone is based on Faraday's law of induction, which is expressed in the Laplace domain in equation (D.1). Here, $S_{g}$ is the geophone sensitivity $(\mathrm{V} /(\mathrm{m} / \mathrm{s}))$ and $\Delta X_{s}$ is the displacement of the seismic mass in the geophone.

$$
V_{g}(s)=-S_{g} s \Delta X_{s}(s)
$$

In section 6.1.1, an expression is derived for $\Delta X_{s}(s)$ as a function of the acceleration $s^{2} X_{b}(s)$ of the object under test and the (change of) orientation $\Theta(s)$ in the gravitational field (equation (6.3)). Here, these effects will be combined into a single apparent acceleration $s^{2} X_{b}$.

Moreover, it will proof useful later on to include the effect of a dynamic (noise) force input $F_{n}(s)$ as well. The resulting expression for $\Delta X(s)$ is presented in equation (D.2). Here, $m$ is the seismic mass, $\zeta_{s}$ is the damping ratio of the geophone resonance frequency, which, in turn, is denoted by $\omega_{s}(\mathrm{rad} / \mathrm{s})$.

$$
\Delta X_{s}(s)=-\frac{s^{2} X_{b}(s)}{s^{2}+2 \zeta_{s} \omega_{s} s+\omega_{s}^{2}}+\frac{1}{m} \frac{F_{n}(s)}{s^{2}+2 \zeta_{s} \omega_{s} s+\omega_{s}^{2}}
$$


The geophone responses to base acceleration and an internal force are then given by the transfer functions shown in equations (D.3) and (D.4).

$$
\begin{aligned}
& H_{g}(s) \equiv \frac{V_{g}(s)}{s^{2} X_{b}(s)}=\frac{S_{g} s}{s^{2}+2 \zeta_{s} \omega_{s} s+\omega_{s}^{2}} \\
& H_{F}(s) \equiv \frac{V_{g}(s)}{F_{n}(s)}=-\frac{S_{g} s}{m\left(s^{2}+2 \zeta_{s} \omega_{s} s+\omega_{s}^{2}\right)}
\end{aligned}
$$

The tested geophone is a GeoSpace GS-11D. An overview of the parameter values for this geophone is presented in table D.1.

Table D.1: GeoSpace GS-11D model parameters

\begin{tabular}{|c|r|}
\hline Parameter & Value \\
\hline$S_{g}$ & $32 \mathrm{~V} /(\mathrm{m} / \mathrm{s})$ \\
\hline$\omega_{s}$ & $2 \pi \cdot 4.5 \mathrm{rad} / \mathrm{s}$ \\
\hline$\zeta_{s}$ & $0.34-$ \\
\hline$R_{c}$ & $380 \Omega$ \\
\hline$L_{c}$ & $50 \mathrm{mH}$ \\
\hline$m$ & $0.0236 \mathrm{~kg}$ \\
\hline
\end{tabular}

The small velocity levels ${ }^{1}$ make additional signal amplification necessary. Figure D.1 shows the three-stage amplifier circuit that was used. The geophone is modelled in the electrical domain as a voltage source $V_{g}$ in series with the coil resistance $R_{c}$. The coil inductance $L_{c}$ is not significant in the frequency range of interest $(0-1 \mathrm{kHz})$, see also table D.1.

The OP27 operational amplifiers (opamps) serve as input and output buffers, thus eliminating the sensitivity of the amplifier stage to source and load impedances. The OP37 opamp is placed in an inverting gain stage, combined with a first-order low-pass filter. Assuming an ideal opamp ${ }^{2}$, the (dynamic) inverting gain $G_{I}(s)$ of the amplifier stage is given by equation (D.5).

$$
G_{I}(s)=-\frac{R_{f}}{R_{i}} \frac{1}{1+s C_{f} R_{f}}
$$

\footnotetext{
1 A VC-E input level corresponds to approx. $15 \mu \mathrm{m} / \mathrm{s}$ RMS $(0-1 \mathrm{kHz})$

2 infinite gain and bandwidth, infinite input impedance and zero output impedance
} 


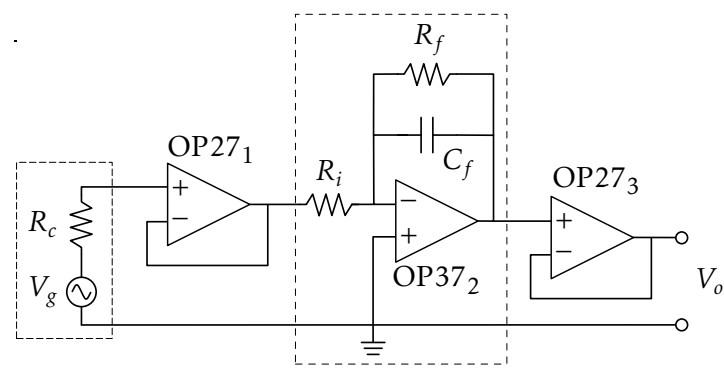

Figure D.1: Geophone electrical model (dashed,left) and amplifier circuit (input, output buffers and amplifier stage (dashed, middle))

\section{D.2 Noise analysis}

The following noise sources can be identified in the geophone and the amplifier circuit:

- thermo-mechanical noise (Brownian motion of the seismic mass)

- thermo-electrical noise in $R_{c}, R_{i}$ and $R_{f}$ (Johnson-Nyquist noise)

- dielectric loss in $C_{f}$

- noise in the opamps OP27/OP37

The thermo-mechanical noise can be modelled by the internal force $F_{n}$. The power spectral density (PSD) $P_{F_{n} F_{n}}$ of $F_{n}$ is then independent of frequency and given by equation (D.6). Here, $T$ is the absolute temperature (K) and $k_{B}$ is Boltzmann's constant $\left(1.38 \cdot 10^{-23} \mathrm{~J} / \mathrm{K}\right)$.

$$
P_{F_{n} F_{n}}=4 k_{B} T 2 m \zeta_{s} \omega_{s} \quad\left[\mathrm{~N}^{2} / \mathrm{Hz}\right]
$$

The thermo-electrical noise of resistors can be modelled similarly as a voltage source with PSD $P_{R R}$ in series with the resistor, see equation (D.7).

$$
P_{R R}=4 k_{B} T R
$$$$
\left[\mathrm{V}^{2} / \mathrm{Hz}\right]
$$

The dielectric losses in a capacitor $C$ can be modelled by placing a resistor $R_{p}$ (with equivalent noise voltage source) in parallel to the capacitor. The equivalent parallel resistor is frequency dependent and depends on the loss factor $\eta$, see equation (D.8).

$$
R_{p}(\omega)=\frac{1}{\eta \omega C}
$$




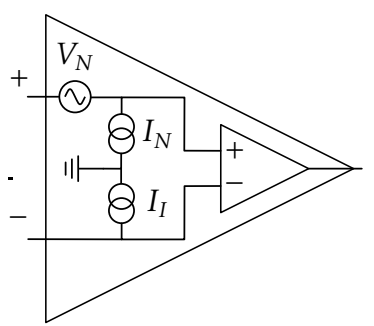

Figure D.2: Equivalent noise model of an operational amplifier

The noise in opamps is commonly modelled as a current noise source $I_{I}$ at the inverting input of an ideal opamp and a current noise source $I_{N}$ and a voltage noise source $V_{N}$ at the non-inverting input of the ideal opamp, see figure D.2.

The power spectral densities of the current and voltage noise are modelled as a combination of $1 / f$-noise and white noise, see equations (D.9) and (D.10).

$$
\begin{aligned}
& P_{I I}(f)=P_{I I, \infty}\left(1+\frac{f_{c I}}{f}\right) \\
& P_{V V}(f)=P_{V V, \infty}\left(1+\frac{f_{c V}}{f}\right)
\end{aligned}
$$

The current noise sources $I_{I}$ and $I_{N}$ are assumed to have identical PSDs. Table D.2 lists typical and maximum values for the noise corner frequencies $f_{c I}, f_{c V}$ and the white noise levels $P_{I I, \infty}$ and $P_{V V, \infty}$ [Analog Devices $\left.(2006,2002)\right]$.

Table D.2: Noise parameters for OP27/37(G) opamps

\begin{tabular}{|c|c|c|l|}
\hline Parameter & Typ. & Max. & Units \\
\hline$P_{V V, \infty}$ & 9 & 20 & $(\mathrm{nV})^{2} / \mathrm{Hz}$ \\
\hline$f_{c V}$ & 2.7 & 24 & $\mathrm{~Hz}$ \\
\hline$P_{I I, \infty}$ & 0.16 & 0.36 & $(\mathrm{pA})^{2} / \mathrm{Hz}$ \\
\hline$f_{c I}$ & 140 & 250 & $\mathrm{~Hz}$ \\
\hline
\end{tabular}

Using basic circuit analysis techniques [Texas Instruments (2007)], the contribution of each noise source to the output voltage is evaluated. For this analysis, it is assumed that the opamps are ideal (infinite gain, infinite input impedance, zero output impedance). It is also possible to include these non-ideal opamp properties in the analysis, although a computational solution method is then advised, see e.g. [Ho et al. (1975); Wedepohl and Jackson (2002)]. 
The results are listed in table D.3. The first column indicates the noise source, the second column gives the transfer function to the output and the third column gives the PSD of the noise source. In the last column, a short description of each noise source is given. For ease of notation in the table, the opamps in figure D.1 are numbered 1-3, from left to right.

Table D.3: Noise analysis results for the circuit in figure D.1

\begin{tabular}{|c|c|c|c|}
\hline $\mathbf{u}(\mathbf{s})$ & $\frac{V_{\mathbf{o}}(s)}{u(s)}$ & $\operatorname{PSD}(\mathbf{u})$ & Description/notes \\
\hline$s^{2} X_{b}(s)$ & $G_{I}(s) H_{g}(s)$ & - & $\begin{array}{c}\text { Nominal response } \\
\text { to acceleration }\end{array}$ \\
\hline$F_{n}(s)$ & $G_{I}(s) H_{F}(s)$ & $4 k_{B} T 2 m \zeta_{S} \omega_{s}$ & Thermo-mech. noise \\
\hline$V_{R_{c}}(s)$ & $G_{I}(s)$ & $4 k_{B} T R_{c}$ & Thermo-electr. noise in $R_{c}$ \\
\hline$V_{R_{i}}(s)$ & $G_{I}(s)$ & $4 k_{B} T R_{i}$ & Thermo-electr. noise in $R_{i}$ \\
\hline$V_{R_{f}}(s)$ & $\frac{1}{1+s C_{f} R_{f}}$ & $4 k_{B} T R_{f}$ & Thermo-electr. noise in $R_{f}$ \\
\hline$V_{C_{f}}(s)$ & $\approx-\frac{G_{I}(s)}{R_{p}}$ & $4 k_{B} T R_{p}$ & Dielectric loss in $C_{f}$ \\
\hline$V_{N_{1}}(s)$ & $G_{I}(s)$ & eqn. (D.10) & Voltage noise of opamp 1 \\
\hline$I_{N_{1}}(s)$ & $G_{I}(s)\left(R_{c}-S_{g} H_{F}(s)\right)$ & eqn. (D.9) & $\begin{array}{l}\text { Current noise at non-inver- } \\
\text { ting input of opamp } 1\end{array}$ \\
\hline$I_{I_{1}}(s)$ & 0 & eqn. (D.9) & $\begin{array}{l}\text { Current noise at inver- } \\
\text { ting input of opamp } 1\end{array}$ \\
\hline$V_{N_{2}}(s)$ & $1-G_{I}(s)$ & eqn. (D.10) & Voltage noise of opamp 2 \\
\hline$I_{N_{2}}(s)$ & 0 & eqn. (D.9) & $\begin{array}{l}\text { Current noise at non-inver- } \\
\text { ting input of opamp } 2\end{array}$ \\
\hline$I_{I_{2}}(s)$ & $-\frac{R_{f}}{1+s C_{f} R_{f}}$ & eqn. (D.9) & $\begin{array}{l}\text { Current noise at inver- } \\
\text { ting input of opamp } 2\end{array}$ \\
\hline$V_{N_{3}}(s)$ & 1 & eqn. (D.10) & Voltage noise of opamp 3 \\
\hline$I_{N_{3}}(s)$ & 0 & eqn. (D.9) & $\begin{array}{c}\text { Current noise at non-inver- } \\
\text { ting input of opamp } 3\end{array}$ \\
\hline$I_{I_{3}}(s)$ & 0 & eqn. (D.9) & $\begin{array}{l}\text { Current noise at inver- } \\
\text { ting input of opamp } 3\end{array}$ \\
\hline
\end{tabular}


After all the noise contributions to the output signal have been determined, the equivalent acceleration PSD can be computed from the total output noise PSD $P_{V_{o} V_{o}}$, see equation (D.11).

$$
P_{\ddot{x}_{b} \ddot{x}_{b}}(f)=\frac{P_{V_{o} V_{o}}(f)}{\left|G_{I}(f)\right|^{2}\left|H_{g}(f)\right|^{2}}
$$

The passive components in the amplifier stage are chosen as follows: $R_{f}=$ $10 \mathrm{k} \Omega, R_{i}=100 \Omega, C_{f}=8.2 \mathrm{nF}$, resulting in a DC gain $G_{I}(0)$ of -100 and a single pole at $1.9 \mathrm{kHz}$.

Due to the high gain setting, the noise sources whose contributions scale by $G_{I}(s)$ will dominate. Hence, the other noise sources can be neglected. Moreover, the dielectric loss in $C_{f}$ has a negligible contribution for $\eta=0.01$ (estimated). Lastly, because $R_{i}$ is much smaller than $R_{c}$, the thermo-electrical noise contribution of $R_{i}$ is discarded as well.

Then, five significant noise sources remain. These are the thermo-mechanical noise, the thermo-electrical noise in $R_{c}$, the voltage noise of the first opamp $V_{N_{1}}$, the current noise $I_{N_{1}}$ of the first opamp and the voltage noise of the second opamp $V_{\mathrm{N}_{2}}$.

In figure D.3, the equivalent acceleration PSDs of these noise sources are plotted, as well as the total equivalent acceleration PSD. In this figure, the typical noise values of the OP27/37 opamps have been used. Clearly, the voltage noise of the first two opamps $\left(V_{N_{1}}\right.$ and $\left.V_{N_{2}}\right)$ dominate the total noise, except at the geophone's resonance frequency. At resonance, thermo-mechanical noise and current noise through the coil become the most dominant contributions.

In figure D.4, the results with the maximum noise values for the opamps are presented. Below the resonance frequency, the total PSD level is increased by an order of magnitude. At high frequencies, the total PSD level is roughly doubled. In this case, the voltage noise contributions of the opamps are the dominant noise source over the entire frequency range. This is exactly the reason why the OP27/37 opamps have been selected, as they have low voltage noise levels. 


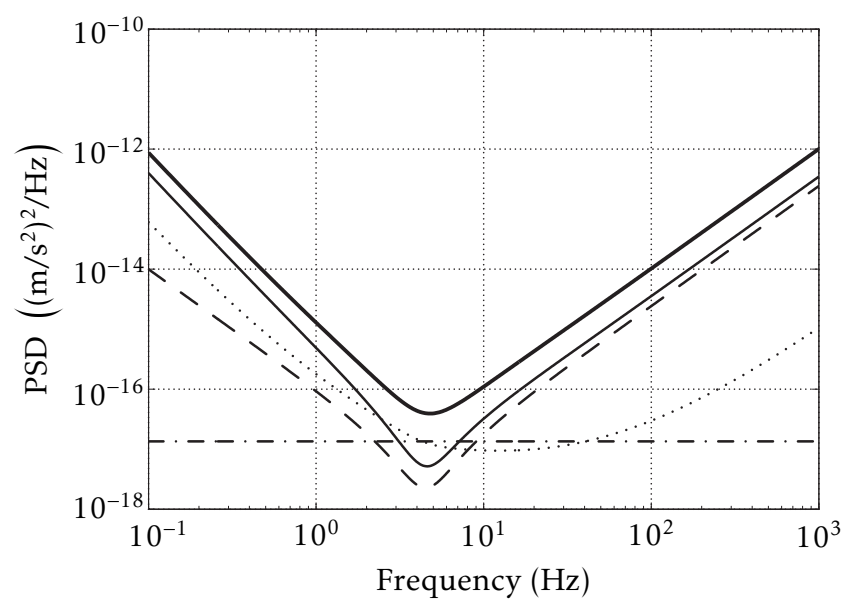

Figure D.3: Equivalent acceleration PSD of the dominant noise sources in the geophone circuit of figure D.1, for typical opamp noise levels.

- : Total equivalent acceleration

-.-: Thermo-mechanical noise

- - : Thermo-electrical noise in $R_{c}$

$\cdots$ : Current noise $I_{N_{1}}$

- Voltage noise $V_{N_{1}}\left(V_{N_{2}}\right.$ curve is overlaid on $V_{N_{1}}$ curve)

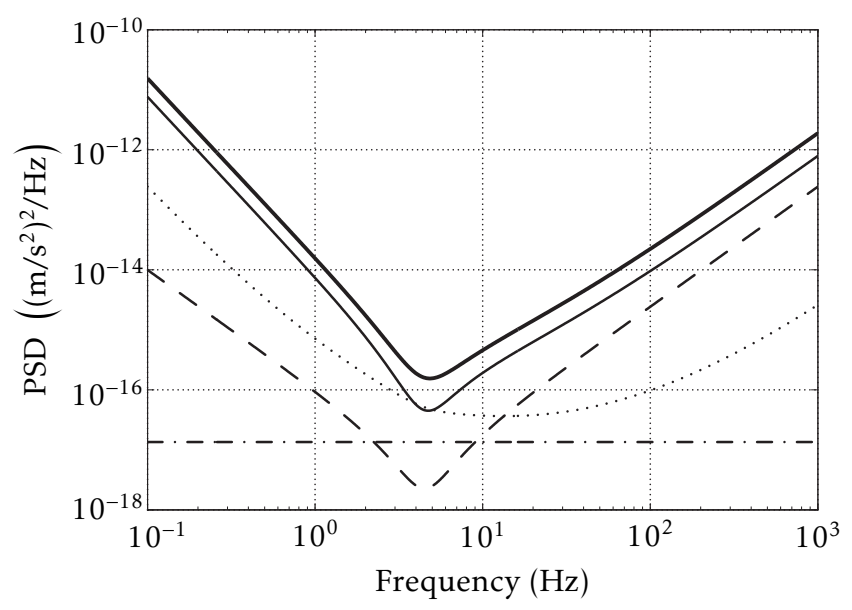

Figure D.4: Same as above, but for maximum opamp noise levels. 



\section{Appendix E}

\section{Frequency domain subspace identification}

In this appendix, the frequency domain system identification method is described that is used in this thesis. The theory of frequency domain identification is taken from [Pintelon and Schoukens (2001)]. Moreover, the subspace identification algorithm that is used to estimate the state-space matrices is taken from [McKelvey et al. (1996)].

\section{E.1 System identification procedure}

In general, the system identification process may include the following five ${ }^{1}$ steps:

1. Model structure selection

2. Experiment design

3. Data acquisition and preprocessing

4. Model parameter estimation

5. Model validation

These steps are discussed separately in sections E.2-E.6.

1 Note that there is no agreement on these steps in the system identification literature, see e.g. [Ljung (1999); Pintelon and Schoukens (2001)] 


\section{E.2 Model structure selection}

In this thesis, a linear, time-invariant, discrete-time state space system representation is used, see equation (E.1). The vectors $\boldsymbol{x}(k), \boldsymbol{u}(k)$ and $\boldsymbol{y}(k)$ are, respectively, the state, input and output vectors at sample interval $k$. The matrices $\boldsymbol{A}, \boldsymbol{B}, \boldsymbol{C}$ and $\boldsymbol{D}$ have real coefficients and are suitably sized.

$$
\begin{aligned}
\boldsymbol{x}(k+1) & =\boldsymbol{A} \boldsymbol{x}(k)+\boldsymbol{B} \boldsymbol{u}(k) \\
\boldsymbol{y}(k) & =\boldsymbol{C} \boldsymbol{x}(k)+\boldsymbol{D} \boldsymbol{u}(k)
\end{aligned} \quad \boldsymbol{x} \in \mathbb{R}^{N_{x}}, \boldsymbol{u} \in \mathbb{R}^{N_{u}}, \boldsymbol{y} \in \mathbb{R}^{N_{y}}
$$

The state-space description in equation (E.1) is fully parametrized, i.e. it uses the maximum of $N_{x}^{2}+N_{x}\left(N_{y}+N_{u}\right)+N_{y} N_{u}$ parameters for its matrices. It can be shown that in general only $N_{x}\left(N_{y}+N_{u}\right)+N_{y} N_{u}$ (independent) parameters are required to describe the system's input-output behaviour. Therefore, the fully parametrized state-space description is not identifiable. However, identifiability of the parameter set is not the major interest here. Instead, we are interested in an accurate representation of the input-output behaviour. For this purpose, the fully parametrized state-space description is also suited.

\section{E.3 Experiment design}

Figure E.1 shows a general block diagram of the experimental setup. $G(z)$ represents the true system with $N_{u}=2$ inputs and $N_{y}=3$ outputs. It is assumed that the input sequence $\boldsymbol{u}(k)$ is computer-generated and therefore directly available (without any noise contribution). The measurement sequence $y(k)$ is corrupted by noise $\boldsymbol{n}(k)$. All the noise sources and external disturbances are incorporated in the noise sequence $\boldsymbol{n}(k)$.

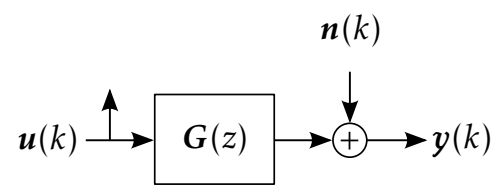

Figure E.1: General block diagram of the measurement setup 


\section{E.3.1 Design of the input sequences}

In this thesis, we choose to excite the system with each actuator separately during each experiment. The input sequences for each channel are chosen to be random phase multisines, which are periodic signals. The use of periodic excitations has some clear advantages, as discussed in [Pintelon and Schoukens (2001), Ch. 2, 3 and 10]:

- The system response to the excitation signal is periodic, whereas the noise contributions are generally not. As a result, by averaging over $P$ periods, the noise influence is reduced.

- The noise covariance can be estimated from the $P$ measured periods as well. The covariance estimate can be used to improve the system identification results.

- In case of frequency domain identification, the frequency data set can be confined to the excited frequencies only, i.e. for each excited frequency only 2 data points (e.g. amplitude and phase) need to be stored for each signal. This commonly results in a significant data reduction compared to the measured time traces.

Equation (E.2) expresses a scalar random phase multi-sine signal $v(t)$ with $N_{f}$ components. The phases $\varphi_{l}$ are a realization of an independent (over $l$ ) distributed random process on $(-\pi, \pi)$, such that $\mathrm{E}\left\{\mathrm{e}^{j \varphi_{l}}\right\}=0$. The excited frequencies $f_{l}$ are integer multiples of the base frequency $f_{b}$.

$$
v(t)=\sum_{l=1}^{N_{f}} A_{l} \cos \left(2 \pi f_{l} t+\varphi_{l}\right), \quad f_{l}=k_{l} f_{b}, \quad k_{l} \in \mathbb{N}
$$

The user has extensive control over the excitation signal, i.e. the base frequency $f_{b}$, the amplitude spectrum $A_{l}$ and the excited frequency grid $f_{l}$ are user-definable. It is strongly advised to relate the base frequency $f_{b}$, the sampling frequency $f_{s}$ and the number of measured samples per period $M$ according to equation (E.3). ${ }^{2}$ In this case, the Discrete Fourier Transform (DFT) of the periodic data does not suffer from leakage errors.

$$
M f_{b}=f_{s}
$$

For later reference, the normalized excited frequencies $\omega_{l}^{e}$ are defined in equation (E.4).

$$
\omega_{l}^{e}=\frac{2 \pi f_{l}}{f_{s}}=\frac{2 \pi k_{l}}{M}, \quad l=1, \ldots, N_{f}, \quad 2 k_{l} \leq M
$$

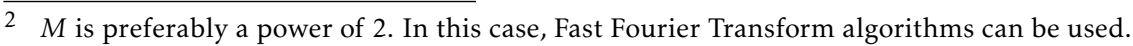




\section{E.4 Data acquisition and preprocessing}

After the measurement data have been collected for the various experiments (one for each input channel), the measured time data is preprocessed to produce a non-parametric estimate of the frequency response function (FRF) of $\boldsymbol{G}(z)$. Moreover, the covariance matrix of the FRF is estimated.

The system identification algorithm that is used in this thesis requires a nonparametric estimate of the FRF and an estimate of the FRF covariance. The subspace identification method is discussed in section E.5.

\section{E.4.1 Estimation of the non-parametric FRF}

Let $\boldsymbol{Y}^{(e)[p]}\left(\omega_{m}\right)$ denote the (vectorized) DFT of the output sequence for period $p$ of experiment $e$ at the normalized frequency $\omega_{m}$, see equation (E.5). The average is removed from the time sequences $y^{(e)}(k)$ prior to computing the DFT.

It is strongly advised to compensate for any pure delays from the output DFT spectra, by multiplying the DFT spectra at each frequency $\omega_{m}$ by $\mathrm{e}^{j d \omega_{m}}$ where $d$ is the number of delays. These delays can later be easily re-applied to the identified model.

$$
\begin{aligned}
& \boldsymbol{Y}^{(e)[p]}\left(\omega_{m}\right)=\sum_{k=0}^{M-1} y^{(e)}(k) \mathrm{e}^{-j k \omega_{m}}, \quad p=1, \ldots, P \\
& \omega_{m}=\frac{2 \pi m}{M}, \quad m=0, \ldots, M-1
\end{aligned}
$$

For each experiment $e$, the mean of the output DFT spectra is estimated at the excited frequencies $\omega_{l}^{e}$ according to equation (E.7). Because the input sequence for each experiment is user-defined and exactly known, it suffices to compute the input DFT spectrum $\hat{\boldsymbol{U}}^{(e)}\left(\omega_{l}^{e}\right)$ for a single period.

$$
\hat{\boldsymbol{Y}}^{(e)}\left(\omega_{l}^{e}\right)=\frac{1}{P} \sum_{p=1}^{P} \boldsymbol{Y}^{(e)[p]}\left(\omega_{l}^{e}\right)
$$

By combining the mean output DFT spectra for all $N_{e}$ experiments, the output data matrix $\mathcal{Y}$ is formed, see equation (E.8). The input data matrix $\mathcal{U}$ and the noise data matrix $\mathcal{N}$ are defined in a similar way.

$$
\mathcal{Y}\left(\omega_{l}^{e}\right)=\left[\begin{array}{lll}
\hat{\boldsymbol{Y}}^{(1)}\left(\omega_{l}^{e}\right) & \cdots & \hat{\boldsymbol{Y}}^{\left(N_{e}\right)}\left(\omega_{l}^{e}\right)
\end{array}\right] \in \mathbb{C}^{N_{y} \times N_{e}}
$$


The combined data equation (see also figure E.1), evaluated at the normalized excited frequency $\omega_{l}$, is then given by equation (E.9). Here, $\boldsymbol{G}\left(\omega_{l}^{e}\right)$ is the (complex-valued) frequency response of $\boldsymbol{G}(z)$ at frequency $\omega_{l}^{e}$.

$$
\mathcal{Y}\left(\omega_{l}^{e}\right)=\boldsymbol{G}\left(\omega_{l}^{e}\right) \mathcal{U}\left(\omega_{l}^{e}\right)+\mathcal{N}\left(\omega_{l}^{e}\right)
$$

When exactly $N_{u}$ experiments are performed, a non-parametric estimate $\hat{\boldsymbol{G}}\left(\omega_{l}^{e}\right)$ of the FRF $\boldsymbol{G}\left(\omega_{l}^{e}\right)$ can be computed by equation (E.10). Under the assumption that the noise is circular complex normally distributed, $\hat{\boldsymbol{G}}\left(\omega_{l}^{e}\right)$ is the maximum-likelihood estimate of $\boldsymbol{G}\left(\omega_{m}\right)$ [Verboven et al. (2006)].

$$
\hat{\boldsymbol{G}}\left(\omega_{l}^{e}\right)=\mathcal{Y}\left(\omega_{l}^{e}\right) \mathcal{U}^{-1}\left(\omega_{l}^{e}\right)
$$

The FRF estimate (E.10) can only be computed when the inverse of $\mathcal{U}\left(\omega_{l}^{e}\right)$ exists, i.e. $\mathcal{U}\left(\omega_{l}^{e}\right)$ has rank $N_{u}$. Therefore, at least $N_{u}$ experiments need to be performed. Moreover, the input sequences of the various experiments need to be sufficiently different. For example, consider a two-input system, i.e. $N_{u}=2$. Then, the leftmost excitation design in equation (E.11) clearly fails the rank condition, whereas the rightmost design has full rank (when $U\left(\omega_{m}\right) \neq 0$ ). Here, $U\left(\omega_{m}\right)$ is the value of the DFT spectrum of some scalar input sequence at frequency $\omega_{m}$.

Note that, when $N_{e}>N_{u}$, the Moore-Penrose pseudo-inverse of $\mathcal{U}\left(\omega_{l}^{e}\right)$ should be used in equation (E.10) instead of the matrix inverse.

$$
\mathcal{U}\left(\omega_{m}\right)=\left[\begin{array}{ll}
1 & 1 \\
0 & 0
\end{array}\right] U\left(\omega_{m}\right) \quad \mathcal{U}\left(\omega_{m}\right)=\left[\begin{array}{ll}
1 & 0 \\
0 & 1
\end{array}\right] U\left(\omega_{m}\right)
$$

Lastly, note that the total number of excited frequencies must be large enough (in relation to the system order $N_{x}$ ), i.e. a sufficiently dense frequency grid should be chosen for the multisine signal. This condition is commonly referred to as persistence of excitation, see also section E.5.

\section{E.4.2 Estimation of the covariance of the non-parametric FRF}

The covariance of the mean output DFT spectra is estimated according to equation (E.12). The additional factor $1 / P$ in equation (E.12) is due the averaging over the periods. Moreover, $(\bullet)$ is a shorthand notation for the preceding term in parentheses.

Note that, because the input sequences are exactly known, the covariance matrix of the input DFT spectra is identical to zero.

$$
\hat{\boldsymbol{C}}_{\hat{\boldsymbol{Y}}}^{(e)}\left(\omega_{l}^{e}\right)=\frac{1}{P(P-1)} \sum_{p=1}^{P}\left(\boldsymbol{Y}^{(e)[p]}\left(\omega_{l}^{e}\right)-\hat{\boldsymbol{Y}}^{(e)}\left(\omega_{l}^{e}\right)\right)(\bullet)^{\mathrm{H}}
$$


When the input channels are excited separately, as is the case in this thesis, an estimate of the covariance matrix of the FRF estimate can be computed from equation (E.13). For a general expression for the FRF covariance matrix, see e.g. [Verboven et al. (2006)].

$$
\hat{\boldsymbol{C}}_{\hat{\boldsymbol{G}}}\left(\omega_{l}^{e}\right)=\sum_{e=1}^{N_{e}} \hat{\boldsymbol{C}}_{\hat{\boldsymbol{G}}}^{(e)}\left(\omega_{l}^{e}\right)=\sum_{e=1}^{N_{e}}\left\|\hat{\boldsymbol{U}}^{(e)}\left(\omega_{l}^{e}\right)\right\|_{2}^{-2} \hat{\boldsymbol{C}}_{\hat{\boldsymbol{Y}}}^{(e)}\left(\omega_{l}^{e}\right)
$$

A good indicator for the quality of the measured data (and therefore the expected model quality) is the frequency-dependent signal-to-noise ratio (SNR) for each experiment $e$, as defined in equation (E.14). Here, the element-wise division operator is denoted by ./. Because in each experiment only one input channel is excited, these signal-to-noise ratios apply directly to the system transfer functions.

$$
\operatorname{SNR}^{(e)}\left(\omega_{l}^{e}\right)=10 \log _{10}\left(\operatorname{diag}\left(\hat{\boldsymbol{G}}^{(e)}\left(\omega_{l}^{e}\right) \hat{\boldsymbol{G}}^{(e)}\left(\omega_{l}^{e}\right)^{\mathrm{H}}\right) \cdot / \operatorname{diag}\left(\hat{\boldsymbol{C}}_{\hat{\mathbf{G}}}^{(e)}\left(\omega_{l}^{e}\right)\right)\right)
$$

\section{E.5 Subspace identification}

For the estimation of the state-space model parameters, a frequency domain subspace-based algorithm is used [McKelvey et al. (1996), Algorithm 2]. The interested reader is referred to the original paper for further details and proofs.

The algorithm aims at estimating a minimal state-space model, see equation (E.15), by reconstructing certain subspaces which are related to the system matrices. A minimal system is both observable and controllable, which means that both the observability matrix $\mathcal{O}$ and the controllability matrix $\mathcal{R}$ have full rank $N_{x}$, see equations (E.16) and (E.17).

$$
\begin{aligned}
& \boldsymbol{x}(k+1)=\boldsymbol{A} \boldsymbol{x}(k)+\boldsymbol{B} \boldsymbol{u}(k) \quad \boldsymbol{x} \in \mathbb{R}^{N_{x}}, \boldsymbol{u} \in \mathbb{R}^{N_{u}}, \boldsymbol{y} \in \mathbb{R}^{N_{y}} \\
& \boldsymbol{y}(k)=\boldsymbol{C} \boldsymbol{x}(k)+\boldsymbol{D} \boldsymbol{u}(k) \\
& \mathcal{O}=\left[\begin{array}{c}
\boldsymbol{C} \\
\boldsymbol{C} \boldsymbol{A} \\
\vdots \\
\boldsymbol{C} \boldsymbol{A}^{\mathrm{N}_{x}-1}
\end{array}\right] \\
& \mathcal{R}=\left[\begin{array}{llll}
\boldsymbol{B} & \boldsymbol{A B} & \cdots & \boldsymbol{A}^{N_{x}-1} \boldsymbol{B}
\end{array}\right]
\end{aligned}
$$


The subspace identification algorithm consists of two major steps. Firstly, the estimates for $\boldsymbol{A}$ and $\boldsymbol{C}$ are computed from orthogonal projections of certain data matrices. Then, using these estimates, the $\boldsymbol{B}$ and $\boldsymbol{D}$ matrices are computed from a weighted least-squares fit on the measured data.

\section{E.5.1 Estimation of $\mathrm{A}$ and $\mathrm{C}$}

Let the extended observability matrix $\mathcal{O}_{q}$ be defined by equation (E.18). Here, $q$ is the (user-defined) number of block rows (each of size $N_{y} \times N_{x}$ ) in $\mathcal{O}_{q}$.

$$
\mathcal{O}_{q}=\left[\begin{array}{c}
C \\
C A \\
\vdots \\
C A^{q-1}
\end{array}\right]
$$

Given an estimate $\hat{\mathcal{O}}_{q}$ of the extended observability matrix, the estimates $\hat{C}$ and $\hat{A}$ can be computed according to equation (E.19), where [•] ${ }^{\dagger}$ denotes the left inverse. The indexing applied to $\hat{\mathcal{O}}_{q}$ refers to block rows of size $N_{y} \times N_{x}$. For $\left[\hat{\mathcal{O}}_{q}(1: q-1)\right]^{\dagger}$ to have full rank $N_{x}$, we must in general choose $q>N_{x}$. In this case, the computation of $\hat{A}$ is a least-squares problem. Note that it will suffice to construct the matrix $\hat{\mathcal{O}}_{q}=\mathcal{O}_{q} T$ from the measured data, for any invertible $T .^{3}$

$$
\hat{C}=\hat{\mathcal{O}}_{q}(1), \quad \hat{A}=\left[\hat{\mathcal{O}}_{q}(1: q-1)\right]^{\dagger} \hat{\mathcal{O}}_{q}(2: q)
$$

In the remainder of this section, it is discussed how an estimate of the extended observability matrix can be obtained from the measured frequency response function.

$\bar{T} \boldsymbol{T}$ can be interpreted as a similarity transformation of the state vector. 


\section{Data equation for the measured frequency response data}

The FRF estimate $\hat{\boldsymbol{G}}\left(\omega_{l}^{e}\right)$ is expressed in terms of the true FRF $\boldsymbol{G}\left(\omega_{l}^{e}\right)$ and a noise contribution $N_{G}\left(\omega_{l}^{e}\right)$, see equation (E.20). Moreover, the true FRF can be expressed in terms of the system matrices $\boldsymbol{A}, \boldsymbol{B}, \boldsymbol{C}$ and $\boldsymbol{D}$, see equation (E.21).

$$
\begin{aligned}
& \hat{\boldsymbol{G}}\left(\omega_{l}^{e}\right)=\boldsymbol{G}\left(\omega_{l}^{e}\right)+\boldsymbol{N}_{\boldsymbol{G}}\left(\omega_{l}^{e}\right) \\
& \left.\begin{array}{rl}
\mathrm{e}^{j \omega_{l}^{e}} \boldsymbol{X}\left(\omega_{l}^{e}\right) & =\boldsymbol{A} \boldsymbol{X}\left(\omega_{l}^{e}\right)+\boldsymbol{B} \\
\boldsymbol{G}\left(\omega_{l}^{e}\right) & =\boldsymbol{C} \boldsymbol{X}\left(\omega_{l}^{e}\right)+\boldsymbol{D}
\end{array}\right\} \quad \boldsymbol{X}\left(\omega_{l}^{e}\right) \in \mathbb{C}^{N_{x} \times N_{u}}
\end{aligned}
$$

By using equations (E.20) and (E.21) repeatedly, a large data equation is obtained, see equation (E.22). Here, $\Gamma_{q}$ is a lower triangular block Toeplitz matrix, see equation (E.23).

$$
\begin{gathered}
\underbrace{\left[\begin{array}{c}
\hat{\boldsymbol{G}}\left(\omega_{l}^{e}\right) \\
\mathrm{e}^{j \omega_{l}^{e}} \hat{\boldsymbol{G}}\left(\omega_{l}^{e}\right) \\
\vdots \\
\mathrm{e}^{j(q-1) \omega_{l}^{e}} \hat{\boldsymbol{G}}\left(\omega_{l}^{e}\right)
\end{array}\right]}_{\mathcal{G}\left(\omega_{l}^{e}\right)}=\underbrace{\left[\begin{array}{c}
\boldsymbol{C} \\
\boldsymbol{C A} \\
\vdots \\
\boldsymbol{C} \boldsymbol{A}^{q-1}
\end{array}\right]}_{\boldsymbol{\mathcal { O }}_{q}} \boldsymbol{X}\left(\omega_{l}^{e}\right)+\boldsymbol{\Gamma}_{q} \underbrace{\left[\begin{array}{c}
\boldsymbol{I}_{N_{u}} \\
\mathrm{e}^{j \omega_{l}^{e} \boldsymbol{I}_{N_{u}}} \\
\vdots \\
\mathrm{e}^{j(q-1) \omega_{l}^{e}} \boldsymbol{I}_{N_{u}}
\end{array}\right]}_{\mathcal{I}\left(\omega_{l}^{e}\right)}+\underbrace{\left[\begin{array}{c}
\boldsymbol{N}_{\boldsymbol{G}}\left(\omega_{l}^{e}\right) \\
\mathrm{e}^{j \omega_{l}^{e} \boldsymbol{N}_{\boldsymbol{G}}\left(\omega_{l}^{e}\right)} \\
\vdots \\
\mathrm{e}^{j(q-1) \omega_{l}^{e} \boldsymbol{N}_{\boldsymbol{G}}\left(\omega_{l}^{e}\right)}
\end{array}\right]}_{\mathcal{N}_{\boldsymbol{G}}\left(\omega_{l}^{e}\right)} \\
\boldsymbol{\Gamma}_{q}=\left[\begin{array}{cccc}
\boldsymbol{D} & \mathbf{0} & \cdots & \mathbf{0} \\
\boldsymbol{C B} & \boldsymbol{D} & \cdots & \mathbf{0} \\
\vdots & \vdots & \ddots & \vdots \\
\boldsymbol{C} \boldsymbol{A}^{q-2} \boldsymbol{B} & \boldsymbol{C} \boldsymbol{A}^{q-3} \boldsymbol{B} & \cdots & \boldsymbol{D}
\end{array}\right]
\end{gathered}
$$

Concatenating equation (E.22) horizontally for all excited frequencies $\omega_{l}^{e}$ $\left(l=1, \ldots, N_{f}\right)$, results in the overall data equation (E.24), which is the starting point for the subspace identification algorithm.

$$
\begin{aligned}
\underbrace{\left[\begin{array}{lll}
\mathcal{G}\left(\omega_{1}^{e}\right) & \cdots & \mathcal{G}\left(\omega_{N_{f}}^{e}\right)
\end{array}\right]}_{\mathcal{G} \in \mathbb{C}^{q N_{y} \times N_{u} N_{f}}}=\mathcal{O}_{q} \underbrace{\left[\begin{array}{lll}
\boldsymbol{X}\left(\omega_{1}^{e}\right) & \cdots & \boldsymbol{X}\left(\omega_{N_{f}}^{e}\right)
\end{array}\right]}_{\mathcal{X} \in \mathbb{C}^{N_{x} \times N_{u} N_{f}}}+\cdots \\
\Gamma_{q} \underbrace{\left[\begin{array}{llll}
\mathcal{I}\left(\omega_{1}^{e}\right) & \cdots & \mathcal{I}\left(\omega_{N_{f}}^{e}\right)
\end{array}\right]}_{\mathcal{I} \in \mathbb{C}^{q N_{u} \times N_{u} N_{f}}}+\underbrace{\left[\begin{array}{lll}
\mathcal{N}_{\boldsymbol{G}}\left(\omega_{1}^{e}\right) & \cdots & \mathcal{N}_{\boldsymbol{G}}\left(\omega_{N_{f}}^{e}\right)
\end{array}\right]}_{\mathcal{N}_{\boldsymbol{G}} \in \mathbb{C}^{q N_{y} \times N_{u} N_{f}}}
\end{aligned}
$$


From equation (E.24), it is recognized that the data equation contains the extended observability matrix, but that there are also contributions by two other terms $\mathcal{I}$ and $\mathcal{N}_{G}$. In the following subsections, it is discussed how these contributions can be coped with.

\section{Removing the influence of $\mathcal{I}$}

The second term in equation (E.24) can be eliminated by an orthogonal projection onto the null space of $\mathcal{I}$, which is denoted by the matrix multiplication $\Pi_{\mathcal{I}}^{\text {row }^{\perp}}$ in equation (E.25). See section E.7 for the definition of this orthogonal projection (and others). The fact that such projections onto subspaces are used is the origin for the naming of the subspace identification methods.

$$
\mathcal{G} \Pi_{\mathcal{I}}^{\text {row }^{\perp}}=\mathcal{O}_{q} \mathcal{X} \Pi_{\mathcal{I}}^{\text {row }^{\perp}}+\mathcal{N}_{G} \Pi_{\mathcal{I}}^{\text {row }^{\perp}}
$$

Disregarding the noise contribution in equation (E.25) for the moment, $\mathcal{G} \Pi_{\mathcal{I}}^{\text {row }}{ }^{\perp}$ can only be used to compute $\hat{\mathcal{O}}_{q}$ when the rank condition (E.26) is satisfied. Necessary and sufficient conditions for the conditions in equation (E.26) to hold are that $\operatorname{rank}(\mathcal{X})=N_{x}$ and no rank cancellations occur in $\mathcal{X} \Pi_{\mathcal{I}}^{\text {row }^{\perp}}$. It can be shown that the rank condition (E.26) is satisfied when the system is controllable and $N_{f} \geq q+N_{x}$.

$$
\begin{aligned}
\operatorname{rank}\left(\mathcal{O}_{q} \mathcal{X} \Pi_{\mathcal{I}}^{\text {row }^{\perp}}\right) & =\operatorname{rank}\left(\mathcal{O}_{q}\right) \quad\left(=N_{x}\right) \quad \Leftrightarrow \\
\operatorname{rank}\left(\mathcal{X} \Pi_{\mathcal{I}}^{\text {row }^{\perp}}\right) & =\operatorname{rank}\left(\mathcal{O}_{q}\right)
\end{aligned}
$$

By using equation (E.21), $\mathcal{X}$ can be expressed by equation (E.27). Note that $\mathcal{X}$ only exists when $\omega_{l}^{e} \notin \operatorname{eig}(A),\left(l=1, \ldots, N_{f}\right)$, i.e. the system must not have any eigenvalues on the unit circle at the excited frequencies.

$$
\mathcal{X}=\left[\begin{array}{lll}
\left(\mathrm{e}^{j \omega_{1}^{e}} \boldsymbol{I}-\boldsymbol{A}\right)^{-1} \boldsymbol{B} & \ldots & \left(\mathrm{e}^{j \omega_{N_{f}}^{e}} \boldsymbol{I}-\boldsymbol{A}\right)^{-1} \boldsymbol{B}
\end{array}\right]
$$

A singular value decomposition can then be used to form an estimate of the extended observability matrix $\hat{\mathcal{O}}_{q}$, see also equation (E.28). The $N_{x}$ left singular vectors $\boldsymbol{U}_{N_{x}}$ (corresponding to the $N_{x}$ non-zero singular values) form a realization of the extended observability matrix.

$$
\mathcal{G} \Pi_{\mathcal{I}}^{\mathrm{row}^{\perp}}=\left[\begin{array}{ll}
\boldsymbol{U}_{N_{x}} & \boldsymbol{U}_{0}
\end{array}\right]\left[\begin{array}{cc}
\Sigma_{N_{x}} & \mathbf{0} \\
\mathbf{0} & \mathbf{0}
\end{array}\right]\left[\begin{array}{c}
\boldsymbol{V}_{N_{x}}^{\mathrm{H}} \\
\boldsymbol{V}_{0}^{\mathrm{H}}
\end{array}\right]
$$




\section{Reducing the noise influence}

In the practical case that the noise contribution in equation (E.25) is present, $\mathcal{G} \Pi_{\mathcal{I}}^{\text {row }^{\perp}}$ usually has full rank $r>N_{x}$. As a result, there are more than $N_{x}$ non-zero singular values. It is then left to the user to choose the appropriate system order. ${ }^{4}$

More importantly, the $N_{x}$ left singular vectors are perturbed by the noise contribution, and in general no longer form a consistent estimate of the column space of the extended observability matrix. In order to reduce the noise influence, the projected data equation (E.25) must be weighted with the covariance information of the noise.

Let $C_{N}\left(\omega_{l}^{e}\right)$ denote the covariance matrix of the noise contribution at frequency $\omega_{l}^{e}$. Note that the covariance matrix of the FRF $\hat{\boldsymbol{C}}_{\hat{\boldsymbol{G}}}\left(\omega_{l}^{e}\right)$ can be used as an estimate for $C_{N}\left(\omega_{l}^{e}\right)$, see also equation (E.13).

Moreover, let $\mathcal{I}_{N_{y}}$ denote the matrix which is constructed similarly to $\mathcal{I}$, but using the identity matrix $\boldsymbol{I}_{N_{y}}$ instead of $\boldsymbol{I}_{N_{u}}$, see also equations (E.22) and (E.24). Lastly, let $\mathcal{K}$ denote a square root of the matrix product given in equation (E.29). ${ }^{5}$

$$
\mathcal{K K}^{\mathrm{H}}=\mathcal{I}_{N_{y}} \operatorname{diag}\left(C_{N}\left(\omega_{1}^{e}\right), \ldots, C_{N}\left(\omega_{N_{f}}^{e}\right)\right) \mathcal{I}_{N_{y}}^{\mathrm{H}}
$$

Then, the weighted projected data equation (E.30) can be used to find a strongly consistent estimate of $\mathcal{K}^{-1} \mathcal{O}_{q}$ [de Moor (1993); McKelvey et al. (1996)]. This requires that the noise is independent, identically distributed (over the frequencies), with zero mean and bounded second and fourth order moments.

$$
\mathcal{K}^{-1} \mathcal{G} \Pi_{\mathcal{I}}^{\text {row }^{\perp}}=\mathcal{K}^{-1} \mathcal{O}_{q} \mathcal{X} \Pi_{\mathcal{I}}^{\text {row }^{\perp}}+\mathcal{K}^{-1} \mathcal{N}{ }_{G} \Pi_{\mathcal{I}}^{\text {row }^{\perp}}
$$

One possible realization of the extended observability matrix is recovered by equation (E.31), where $\boldsymbol{U}_{N_{x}}$ is the matrix containing the $N_{x}$ left singular vectors of the weighted projected data matrix $\mathcal{K}^{-1} \mathcal{G} \Pi_{\mathcal{I}}^{\text {row }}{ }^{\perp}$.

$$
\hat{\mathcal{O}}_{q}=\mathcal{K} \boldsymbol{U}_{N_{x}}
$$

4 When the signal-to-noise ratio is large enough, a singular value plot will show a clear gap between the true singular values and the "noise" singular values.

$5 \mathcal{K}$ may be computed by a (lower) Cholesky decomposition or an eigenvalue decomposition (see e.g. the MATLAB command sqrtm). 


\section{Notes on implementation}

The projection of equation (E.25) can be computed efficiently from the QRdecomposition of yet another data matrix $\mathcal{Z}$, see equation (E.32). Here, $\bullet$ Re denotes the horizontal concatenation of the real and imaginary parts of a matrix, see equation (E.33). This ensures that $\hat{\mathcal{O}}_{q}$ is real-valued, and therefore the estimated system matrices are real-valued as well.

Using equation (E.32), it can be shown that the projected data equation is given by equation (E.34). Moreover, because $Q_{2}^{\mathrm{T}}$ has full rank, it suffices to use $\boldsymbol{R}_{22}^{\mathrm{T}}$ in the singular value decomposition.

$$
\begin{aligned}
& \mathcal{Z}=\left[\begin{array}{ll}
\left(\mathcal{I}^{\operatorname{Re}}\right)^{\mathrm{T}} & \left(\mathcal{G}^{\mathrm{Re}}\right)^{\mathrm{T}}
\end{array}\right]=\left[\begin{array}{ll}
\boldsymbol{Q}_{1} & \boldsymbol{Q}_{2}
\end{array}\right]\left[\begin{array}{cc}
\boldsymbol{R}_{11} & \boldsymbol{R}_{12} \\
\mathbf{0} & \boldsymbol{R}_{22}
\end{array}\right] \\
& \bullet \\
& \operatorname{Re} \equiv\left[\begin{array}{ll}
\operatorname{Re}(\bullet) & \operatorname{Im}(\bullet)
\end{array}\right] \\
& \left(\mathcal{G} \boldsymbol{\Pi}_{\mathcal{I}^{\text {row }^{\perp}}}\right)^{\operatorname{Re}}=\boldsymbol{R}_{22}^{\mathrm{T}} \boldsymbol{Q}_{2}^{\mathrm{T}}
\end{aligned}
$$

It is not guaranteed that the estimated state-space system is stable, i.e. not all eigenvalues of the matrix $\hat{A}$ are guaranteed to have a magnitude strictly less than one. If desired, stability of the system can be enforced by projection of the unstable eigenvalues of $\hat{A}$ inside the unit circle, see [McKelvey et al. (1996)] for details.

\section{Summary of the derived conditions}

In the foregoing analysis several conditions have been derived which are required for the algorithm to work properly. These are summarized below:

- The system must be controllable and observable. This requirement can also be reversed: only the observable and controllable part of the system can be identified.

- The noise on the frequency data must be zero mean, independent identically distributed with bounded second and fourth moments in order to obtain a strongly consistent estimate.

- The noise covariance must be known a priori. In practice, an estimate of the noise covariance can be used.

- The system must not have any eigenvalues on the unit circle at the excited frequencies. When this requirement is not naturally met, it can be enforced by a proper selection of excited frequencies.

- The number of block rows in the data matrices $q$ must be larger than the system order $N_{x}$. Otherwise, the extended observability matrix does not have full rank. 
- The number of excited frequencies $N_{f}$ must be equal to or larger than $q+N_{x}$. Otherwise, the extended observability matrix can not be extracted from the data set. This requirement is the persistence of excitation condition for this particular algorithm.

\section{E.5.2 Estimation of B and D}

Using the estimated matrices $\hat{C}$ and $\hat{A}$, the matrices $\hat{B}$ and $\hat{D}$ can be estimated from the weighted linear least-squares problem (E.35). The least-squares problem should also be expressed in real-valued matrices by using the $\bullet$ Re operator (E.33), resulting in real-valued estimates $\hat{\boldsymbol{B}}$ and $\hat{\boldsymbol{D}}$.

$$
\begin{aligned}
& \hat{\boldsymbol{B}}, \hat{\boldsymbol{D}}=\arg \min \sum_{l=1}^{N_{f}}\left\|\boldsymbol{C}_{\boldsymbol{N}}^{-1 / 2}\left(\omega_{l}^{e}\right)\left[\hat{\boldsymbol{G}}\left(\omega_{l}^{e}\right)-\boldsymbol{G}\left(\omega_{l}^{e}, \boldsymbol{B}, \boldsymbol{D}\right)\right]\right\|_{F}^{2} \\
& \boldsymbol{G}\left(\omega_{l}^{e}, \boldsymbol{B}, \boldsymbol{D}\right)=\hat{\boldsymbol{C}}\left(\mathrm{e}^{j \omega_{l}^{e}} \boldsymbol{I}-\hat{\boldsymbol{A}}\right)^{-1} \boldsymbol{B}+\boldsymbol{D}
\end{aligned}
$$

Note that the inverse of the square root of the covariance matrix is used as the weighing matrix in the least-squares problem. As a result, the data points with the least variance (i.e. least uncertainty) are the most important data points in the fit. An alternative interpretation is that the data points are weighted according to the signal-to-noise ratio.

It is possible to impose certain constraints on the parameters of the matrices $\hat{\boldsymbol{B}}$ and $\hat{D}$. For instance, the matrix $\hat{D}$ can be forced to be zero, thereby eliminating any direct feedthrough. Moreover, it is possible to enforce zero DC-gain. The DC-gain of the system is given by equation (E.37). Note that the DC-gain is linear in $\boldsymbol{B}$ and $\boldsymbol{D}$ (given $\hat{A}$ and $\hat{\boldsymbol{C}}$ ). Therefore, the zero DC-gain constraint is easily incorporated into the least-squares problem.

$$
G(0, B, D)=\hat{C}(I-\hat{A})^{-1} B+D
$$




\section{E.6 Model selection and validation}

The applied algorithm has two user-definable parameters: the number of block rows $q$ and the desired system order $N_{x}$. In [Pintelon and Schoukens (2001), Section 7.14], it is suggested to perform a (large) search over the parameters $\left(q, N_{x}\right)$, searching for the smallest error in the weighed least-squares fit of equation (E.35), with $q$ in the range $\left[1.5 N_{x}, 6 N_{x}\right]$. An a priori estimate of the system order can be obtained from the measured non-parametric frequency response function.

However, the user may not be interested in obtaining the smallest least-squares error. Slightly larger least-squares errors may be allowed when this improves other characteristics of the identified system.

For example, due to the covariance weighing, the least-squares fit of equation (E.35) primarily attempts to minimize the error at frequencies with a large signal-to-noise ratio (SNR). In general, the SNR at (nearly undamped) antiresonance frequencies will be poor compared to other frequencies. Therefore, there is a risk of erroneously identifying minimum phase zeros as nonminimum phase (NMP) or vice versa. As discussed in chapters 4 and 5, NMP zeros can have significant detrimental effects on the performance of the feedback and feedforward control, and should therefore be identified correctly. In this thesis, the identification of the zeros is checked by inspection of the estimated FRF and the FRF of the identified model.

With only two adjustable parameters $q$ and $N_{x}$, the algorithm is easy to use for the inexperienced user. However, due to this restricted control over the algorithm, a very large value for $N_{x}$ must sometimes be chosen to model the relevant system dynamics. In these cases, a subsequent model order reduction may be required, see e.g. [Safonov and Chiang (1989); Safonov et al. (1990); $\mathrm{Gu}$ (2005); Skogestad and Postlethwaite (2005), Ch. 11] for some model reduction techniques. 


\section{E.7 Subspaces in linear algebra}

Let the matrix $Z \in \mathbb{C}^{m \times n}$ define a linear transformation $T: \mathbb{C}^{n} \rightarrow \mathbb{C}^{m}$, i.e. $y=Z x, x \in \mathbb{C}^{n}, y \in \mathbb{C}^{m}$. There are four subspaces associated with the matrix $Z$ :

- the column space (also range or image) im $(Z)$, equation (E.38)

- the left null space (or cokernel) $\operatorname{ker}\left(Z^{\mathrm{H}}\right)$, equation (E.39)

- the row space (or coimage) $\mathrm{im}\left(Z^{\mathrm{H}}\right)$, equation (E.40)

- the null space (or kernel) $\operatorname{ker}(\boldsymbol{Z})$, equation (E.41))

$$
\begin{aligned}
\operatorname{im}(\boldsymbol{Z}) & =\left\{y \in \mathbb{C}^{m} \mid y=\boldsymbol{Z} \boldsymbol{x}, \boldsymbol{x} \in \mathbb{C}^{n}\right\} \\
\operatorname{ker}\left(\boldsymbol{Z}^{\mathrm{H}}\right) & =\left\{y \in \mathbb{C}^{m} \mid \boldsymbol{Z}^{\mathrm{H}} \boldsymbol{y}=\mathbf{0}\right\} \\
\operatorname{im}\left(\boldsymbol{Z}^{\mathrm{H}}\right) & =\left\{\boldsymbol{x} \in \mathbb{C}^{n} \mid \boldsymbol{x}=\boldsymbol{Z}^{\mathrm{H}} \boldsymbol{y}, \boldsymbol{y} \in \mathbb{C}^{m}\right\} \\
\operatorname{ker}(\boldsymbol{Z}) & =\left\{\boldsymbol{x} \in \mathbb{C}^{n} \mid \boldsymbol{Z} \boldsymbol{x}=\mathbf{0}\right\}
\end{aligned}
$$

The subspaces $\operatorname{im}(\boldsymbol{Z})$ and $\operatorname{ker}\left(\boldsymbol{Z}^{\mathrm{H}}\right)$ are orthogonal complements in $\mathbb{C}^{m}$, i.e. $\operatorname{im}(\boldsymbol{Z}) \oplus \operatorname{ker}\left(\boldsymbol{Z}^{\mathrm{H}}\right)=\mathbb{C}^{m}$ and $\operatorname{im}(\boldsymbol{Z}) \cap \operatorname{ker}\left(\boldsymbol{Z}^{\mathrm{H}}\right)=\{\mathbf{0}\}$. The same holds for $\operatorname{im}\left(\boldsymbol{Z}^{\mathrm{H}}\right)$ and $\operatorname{ker}(\boldsymbol{Z})$ in $\mathbb{C}^{n}$.

Assume that $Z \in \mathbb{C}^{m \times n}$ has rank $r \leq n$. Moreover, let the matrix $N \in \mathbb{C}^{m \times r}$ be chosen such that $\operatorname{im}(\boldsymbol{Z})=\operatorname{im}(\boldsymbol{N})$. Then, the orthogonal projection onto the column space of $Z$, denoted by $\Pi_{Z}^{\text {col }}$, is given by equation (E.42).

$$
\Pi_{Z}^{\text {col }}=N\left(N^{\mathrm{H}} N\right)^{-1} N^{\mathrm{H}}
$$

The projection onto the left null space of $Z$, denoted by $\Pi_{Z}^{\text {col }^{\perp}}$, is simply given by $I-\Pi_{Z}^{\text {col }}$. Note that $N$ can be formed by the linearly independent columns of $\boldsymbol{Z}$. Clearly, when $\boldsymbol{Z}$ has full column rank, it suffices to take $N=Z$.

Similarly, the orthogonal projection onto the row space of $Z\left(\Pi_{Z}^{\text {row }}\right)$ is given by $\Pi_{Z^{\mathrm{H}}}^{\mathrm{col}}$ and the orthogonal projection onto the null space by $\Pi_{Z}^{\text {row }} \equiv \boldsymbol{I}-\boldsymbol{\Pi}_{Z}^{\text {row }}$.

Some important properties of these projections are listed in equation (E.43).

$$
\Pi_{Z} \Pi_{Z}=\Pi_{Z}, \quad \Pi_{Z}^{\mathrm{H}}=\Pi_{Z}, \quad \Pi_{Z}^{\text {col }} Z=Z, \quad Z \Pi_{Z}^{\text {row }}=Z
$$




\section{Appendix F}

\section{Notes on controller implementation}

In this appendix, some details concerning the implementation of the feedback controller and the adaptive feedforward controller are discussed. In section F.1, block diagrams detailing the implementation are presented. In section F.2, the pole locations for the IIR parametrizations are listed and it is discussed how the IIR filter is implemented.

\section{F.1 Controller block diagrams}

A top level block diagram of the overall controller implementation is shown in figure F.1. The control signal $u_{a}(k)$ consists of the feedback control signal $u_{\mathrm{FB}}(k)$ and the feedforward compensation signal $u_{\mathrm{FF}}(k)$. The feedback controller FB is simply an LTI system that filters the error signal $e(k)$. The feedforward controller $F F$ computes the feedforward compensation signal from the reference signal $x(k)$. It uses the error signal $e(k)$ to update its controller parameters.

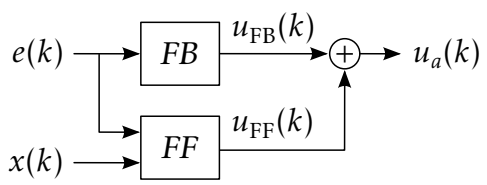

Figure F.1: Block diagram of the controller implementation (top level), showing the feedback controller FB and the (adaptive) feedforward controller FF. 


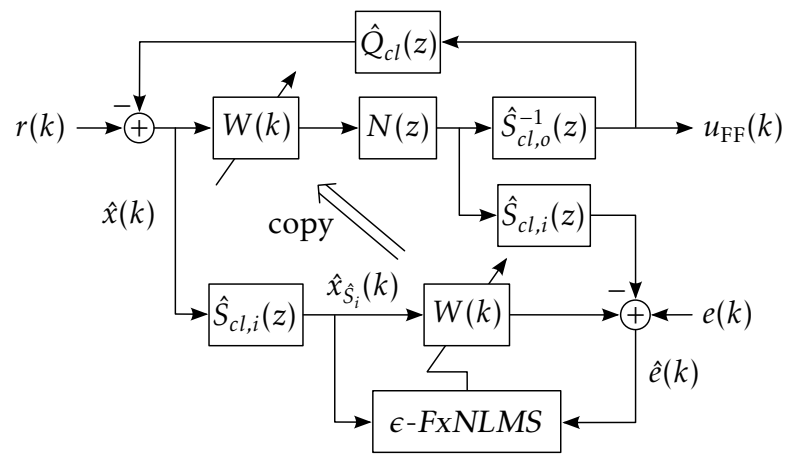

Figure F.2: Block diagram of the adaptive feedforward controller implementation

A detailed block diagram of the implemented adaptive feedforward controller is shown in figure F.2. The model $\hat{Q}_{c l}(z)$ is the internal model used to compensate for the feedback path from the actuator to the reference sensor. The inverse outer factor ${ }^{1} \hat{S}_{c l, o}^{-1}(z)$ is included to precondition the secondary path, leading to faster convergence (see also figure 5.8). The noise shaping filter $N(z)$ is included to shape the control signal in the frequency domain (see also figure 5.10). Lastly, the feedforward controller and the inner factor $\hat{S}_{c l, i}(z)$ are swapped by using the modified FxLMS extension (see figure 5.9). ${ }^{2}$

The controller weights $w(k)$ are updated using a leaky FxLMS update rule with leakage factor $\gamma$ and a normalized step size $\mu(k)$, see equations (F.1) and (F.2). When the feedforward controller has a FIR structure, the regression vector $\psi_{\hat{S}_{i}}(k)$ is given by equation (F.3). For the IIR structure, the regression vector is formed by the states of the second and first order sections, see also section F.2.

$$
\begin{aligned}
& w(k+1)=(1-\gamma) w(k)-\mu(k) \psi_{\hat{S}_{i}}(k) \hat{e}(k), \quad 0<\gamma \ll 1 \\
& \mu(k)=\frac{\bar{\mu}}{\epsilon+\left\|\psi_{\hat{S}_{i}}(k)\right\|_{2}^{2},} \quad 0<\bar{\mu}<2, \quad \epsilon>0 \\
& \psi_{\hat{S}_{i}}(k)=\left[\begin{array}{llll}
\hat{x}_{\hat{S}_{i}}(k) & \hat{x}_{\hat{S}_{i}}(k-1) & \ldots & \hat{x}_{\hat{S}_{i}}(k-L+1)
\end{array}\right]^{\mathrm{T}}
\end{aligned}
$$

To prevent excessively large actuator signals, the closed loop secondary path is augmented with a regularization component $S_{\text {reg }}(z)$, see equation (F.4). By carefully choosing this regularization filter, the spectrum of the augmented secondary path is increased in a selected frequency range.

1 See section C.3 for an implementation of the inner-outer factorization.

2 Note that this swapping and the noise shaping technique require signal operations that cancel each other, leading to a relatively "lean" block diagram. 
As a result, the gain of the outer factor $\hat{S}_{c l, o}(z)$ (which is a spectral factor of the augmented secondary path) is increased in the same frequency range and, consequently, the gain of the inverse of $\hat{S}_{c l, o}(z)$ is reduced. In many cases, it suffices to choose $S_{\text {reg }}(z)=\rho$, where $\rho>0$ is a constant gain.

$$
S_{c l, \text { aug }}(z)=\left[\begin{array}{c}
S_{c l}(z) \\
S_{\text {reg }}(z)
\end{array}\right]
$$

\section{F.2 IIR filter implementation}

The specific IIR filter structure with fixed poles that is considered in this thesis is shown in figure F.3 [Heuberger et al. (2005)]. Its basic structure is a cascaded connection of second order sections (SOS) and first order sections (FOS).

The overall output signal $u(k)$ of the IIR filter is the weighted summation of the states of the second and first order sections, combined with a direct feedthrough term $w_{0}$. Note that the weights $w_{i}^{\text {SOS }}$ for the second order sections are 2-element vectors.

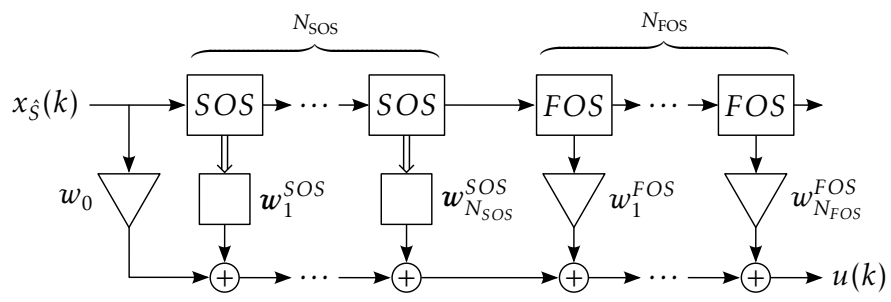

Figure F.3: IIR filter consisting of $N_{S O S}$ second order sections (SOS) and $N_{F O S}$ first order sections (FOS), with possibly adaptive weights.

The structure of the first and second order sections is shown in figure F.4. For either section type, the transfer path from $u_{i}^{\mathrm{F} / \mathrm{SOS}}$ to $y_{\text {all, } i}^{\mathrm{F} / \mathrm{SOS}}$ is an all-pass function. This ensures that the power of the filtered reference signal $x_{\hat{S}}(k)$ is transferred to all sections.

For the first order sections, the parameters of the $i^{\text {th }}$ first order section are related to the pole location $p_{i}$ of that section by equation (F.5).

$$
\left[\begin{array}{ll}
a_{i}^{\mathrm{FOS}} & b_{i}^{\mathrm{FOS}} \\
c_{i}^{\mathrm{FOS}} & d_{i}^{\mathrm{FOS}}
\end{array}\right]=\left[\begin{array}{cc}
p_{i} & \sqrt{1-p_{i}^{2}} \\
\sqrt{1-p_{i}^{2}} & -p_{i}
\end{array}\right]
$$




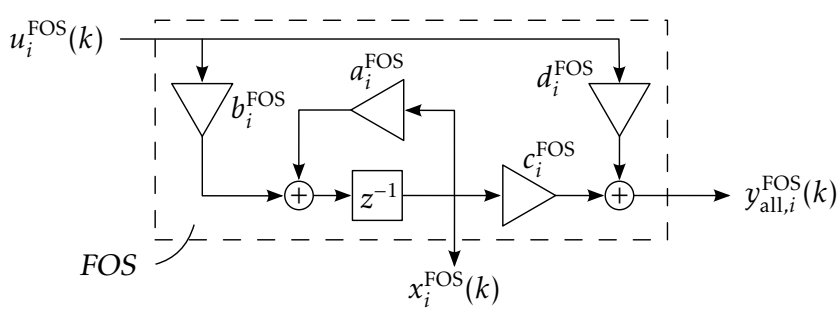

(a) First order section (FOS)

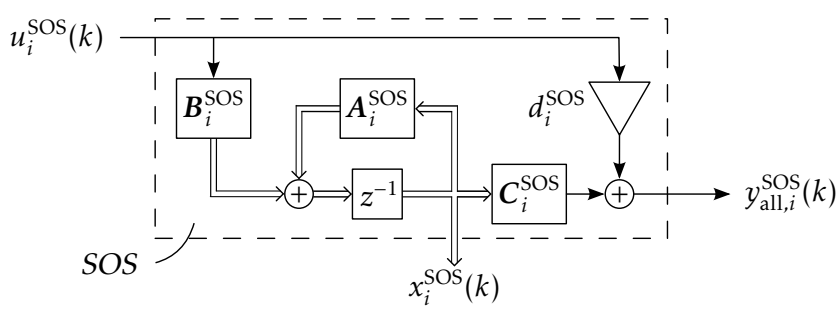

(b) Second order section (SOS)

Figure F.4: Internal structure of the first and second order sections that are used in the IIR filter with fixed poles.

For the second order sections, the parameters of the $i^{\text {th }}$ second order section are related to that section's (complex) pole $p_{i}$ by equations (F.6) and (F.7).

$$
\begin{aligned}
& m_{i}=\frac{2 \operatorname{Re}\left(p_{i}\right)}{1+\left|p_{i}\right|^{2}}, \quad n_{i}=-\left|p_{i}\right|^{2} \\
& {\left[\begin{array}{c|c}
A_{i}^{\mathrm{SOS}} & \boldsymbol{B}_{i}^{\mathrm{SOS}} \\
\hline C_{i}^{\mathrm{SOS}} & d_{i}^{\mathrm{SOS}}
\end{array}\right]=\left[\begin{array}{cc|c}
m_{i} & n_{i} \sqrt{1-m_{i}^{2}} & \sqrt{1-m_{i}^{2}} \sqrt{1-n_{i}^{2}} \\
\sqrt{1-m_{i}^{2}} & -m_{i} n_{i} & -m_{i} \sqrt{1-n_{i}^{2}} \\
\hline 0 & \sqrt{1-n_{i}^{2}} & -n_{i}
\end{array}\right]}
\end{aligned}
$$




\section{IIR pole locations for experiments}

The pole locations for the IIR2 filter are listed in table F.1. The poles marked with $*$ are the pole locations that are used in the IIR1 filter. The experimental results using the IIR1 and IIR2 filters are discussed in section 8.3.5.

Table F.1: Pole locations for the IIR2 filter (poles marked with * are used in the IIR1 filter)

\begin{tabular}{|c|c|c|}
\hline SOS & Freq. $(\mathrm{Hz})$ & $\zeta(\%)$ \\
\hline$-0.8984 \pm 0.3870 j$ & 1393 & 0.806 \\
\hline$-0.9627 \pm 0.08239 j$ & 1557 & 1.13 \\
\hline$-0.9626 \pm 0.08242 j$ & 1557 & 1.13 \\
\hline $0.04084 \pm 0.9710 j$ & 778.7 & 1.87 \\
\hline $0.05715 \pm 0.9856 j$ & 770.5 & 0.847 \\
\hline $0.05797 \pm 0.9720 j$ & 769.8 & 1.76 \\
\hline $0.3280 \pm 0.9334 j$ & 627.9 & 0.871 \\
\hline $0.3280 \pm 0.9333 j$ & 627.9 & 0.873 \\
\hline $0.3408 \pm 0.4311 j$ & 551.3 & 55.3 \\
\hline $0.3597 \pm 0.1601 j$ & 520.5 & 91.2 \\
\hline $0.7259 \pm 0.5783 j$ & $344.7^{*}$ & 11.0 \\
\hline $0.7990 \pm 0.5935 j$ & 325.4 & 0.737 \\
\hline $0.7926 \pm 0.5816 j$ & 322.6 & 2.69 \\
\hline $0.8078 \pm 0.5802 j$ & $317.2^{*}$ & 0.872 \\
\hline $0.9476 \pm 0.3126 j$ & $162.3^{*}$ & 0.686 \\
\hline $0.9396 \pm 0.3102 j$ & 162.5 & 3.30 \\
\hline $0.9101 \pm 0.3899 j$ & $206.2^{*}$ & 2.46 \\
\hline $0.9607 \pm 0.1422 j$ & $76.30^{*}$ & 19.5 \\
\hline $0.8726 \pm 0.4715 j$ & $252.3^{*}$ & 1.667 \\
\hline $0.8485 \pm 0.5028 j$ & $272.6^{*}$ & 2.58 \\
\hline $0.8392 \pm 0.5379 j$ & 290.3 & 0.561 \\
\hline $0.99934 \pm 0.007195 j$ & $3.681^{*}$ & 8.77 \\
\hline $0.8416 \pm 0.5327 j$ & $287.4^{*}$ & 0.712 \\
\hline $0.8416 \pm 0.5327 j$ & 287.4 & 0.711 \\
\hline
\end{tabular}

\begin{tabular}{|c|c|}
\hline FOS & Freq. $\mathbf{( H z )}$ \\
\hline-0.8997 & 1601 \\
\hline-0.7180 & 1609 \\
\hline 0.02807 & 1820 \\
\hline 0.09190 & 1216 \\
\hline 0.5151 & 337.9 \\
\hline 0.6185 & $244.7^{*}$ \\
\hline 0.9074 & $49.50^{*}$ \\
\hline 0.9897 & $5.257^{*}$ \\
\hline 0.9972 & 1.428 \\
\hline 0.99807 & 0.9841 \\
\hline
\end{tabular}




\section{F.3 Transformation to block diagonal form}

In this section, the algorithm is outlined that is used to convert the secondary path state space model $\hat{S}(z)$ to a state space model that has a block-diagonal state matrix $\tilde{A}$. The original state matrix of $\hat{S}(z)$ is denoted by $A$.

Therefore, a change in state variables (from $x$ to $\tilde{\boldsymbol{x}}$, see equation (F.8)) is sought that reduces the matrix $\tilde{A} \equiv T_{d} A T_{d}^{-1}$ to a block diagonal form where the blocks on the diagonal of $\tilde{A}$ represent the eigenvalues of $A$. For real eigenvalues, the corresponding block has size $1 \times 1$ and for complex conjugate eigenvalue pairs the block size is $2 \times 2$. The resulting state-space representation can be used for efficient implementation of the dynamic model in real-time applications.

$$
x \equiv T_{d} \tilde{x}, \quad T_{d} \text { invertible }
$$

The transformation $T_{d}$ can be found in two steps. Firstly, the real Schur decomposition of $A$ is computed, see equation (F.9). Here, $A, S$ and $T_{\mathrm{s}}$ are real, square matrices. $S$ is block upper diagonal, with the eigenvalues of $A$ on its (block) diagonal as specified above. Moreover, $\boldsymbol{T}_{\mathrm{s}}$ is orthonormal, i.e. $T_{s} T_{s}^{\mathrm{T}}=T_{s}^{\mathrm{T}} \boldsymbol{T}_{s}=I$.

$$
A=T_{s} S T_{s}^{\mathrm{T}} \Leftrightarrow S=T_{s}^{\mathrm{T}} A T_{s}
$$

Secondly, $S$ is reduced to block diagonal form using the similarity transformation $T_{b}$, i.e. $\tilde{A}=T_{b} S T_{b}^{-1}$. Under the condition that the system only has (complex conjugate) poles with multiplicity one, the transformation matrix $T_{b}$ can be found using the algorithm outlined below.

1. Partition $S$ as:

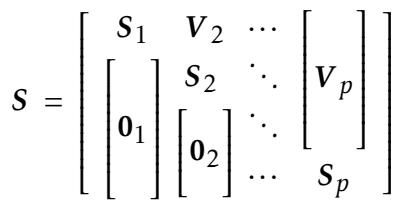

Here, the $S_{i}(i=1, \ldots, p)$ are diagonal blocks (either $1 \times 1$ or $2 \times 2$, depending on the eigenvalue), where $p$ is the total number of $1 \times 1$ - and $2 \times 2$-blocks. $V_{i}(i=2, \ldots, p)$ are corresponding (block) column vectors, which contain all elements above the diagonal block $S_{i}$. Similarly, all the (block) column vectors below the diagonal blocks $\mathbf{0}_{i}(i=1, \ldots, p-1)$ are zero (block) vectors by construction.

2. Initialize the counter $i$ to $p$. 
3. (Re)define $M$ as the square matrix containing the upper left corner of $S$ up to and including $S_{i}$, and partition it as shown:

$$
\boldsymbol{M}=\left[\begin{array}{cc}
\boldsymbol{M}_{11} & \boldsymbol{V}_{i} \\
\mathbf{0} & \boldsymbol{S}_{i}
\end{array}\right]
$$

4. We now seek a transformation matrix $\boldsymbol{T}_{i}$ which solves the following matrix equality:

$T_{i} M=\left[\begin{array}{cc}M_{11} & 0 \\ \mathbf{0} & S_{i}\end{array}\right] T_{i}$

Define the candidate transformation matrix ${ }^{3} \boldsymbol{T}_{i}=\left[\begin{array}{cc}\boldsymbol{I}_{11} & \boldsymbol{X}_{i} \\ \mathbf{0} & \boldsymbol{I}_{i}\end{array}\right]$, where $\boldsymbol{I}_{11}$ and $I_{i}$ are identity matrices of the same size as $\boldsymbol{M}_{11}$ and $S_{i}$, respectively. Then, the matrix inequality is reduced to: $\boldsymbol{M}_{11} \boldsymbol{X}_{i}-\boldsymbol{X}_{i} \boldsymbol{S}_{i}-\boldsymbol{V}_{i}=\mathbf{0}$

The latter equation is a Sylvester equation, which has a unique solution for $\boldsymbol{X}_{i}$ if and only if $\boldsymbol{M}_{11}$ and $\boldsymbol{S}_{i}$ do not share any eigenvalues, i.e. the system must have only (complex conjugate) roots with multiplicity one [Golub et al. (1979)].

5. If $i>2$, decrement $i$ by 1 and return to step 3 .

6. Finally, form the transformation matrix $\boldsymbol{T}_{b}$ :

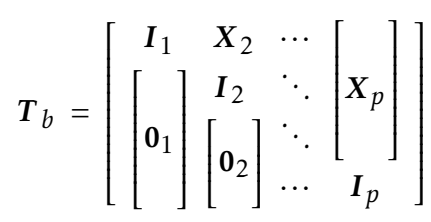

Finally, the complete similarity transformation $\boldsymbol{T}_{d}$ is given by $\boldsymbol{T}_{b} \boldsymbol{T}_{s}^{\mathrm{T}}$. The inverse transformation $\boldsymbol{T}_{d}^{-1}$ is then given by $\boldsymbol{T}_{s} \boldsymbol{T}_{b}^{-1}$. Note that the inverse of $\boldsymbol{T}_{b}$ is easily computed from $T_{b}$, similar to footnote 3 on page 257 .

The real Schur decomposition can be computed using the MATLAB command schur, using the 'real' flag. Moreover, the Sylvester equations can be solved with the MATLAB command lyap.

Note that the algorithm can also be used for systems with poles with multiplicity greater than one, when these poles are grouped into larger diagonal blocks.

3 Note that the inverse of $\boldsymbol{T}_{i}$ is simply given by $\left[\begin{array}{cc}\boldsymbol{I}_{11} & -\boldsymbol{X}_{i} \\ \mathbf{0} & \boldsymbol{I}_{i}\end{array}\right]$ 



\section{Samenvatting}

De werking van productie- en inspectiemachines met hoge nauwkeurigheid kan nadeling beïnvloed worden door trillingen. In veel gevallen zijn vloertrillingen de dominante (mechanische) storingsbron. Om de invloed van deze vloertrillingen te verminderen, wordt gevoelige apparatuur vaak geplaatst op trillingsisolatiesystemen met een relatief lage ondersteuningsstijfheid (zogenaamde soft mounts). Echter, de lage stijfheid kan tot problemen leiden bij excitatie door directe verstoringen (andere verstoringen dan vloertrillingen) en bij het nivelleren van de apparatuur.

Het onderzoek dat beschreven is in dit proefschrift heeft tot doel een alternatief trillingsisolatieconcept te ontwikkelen, dat gebaseerd is op het gebruik van relatieve stijve ondersteuning (zognenaamde hard mounts). De hogere mechaniscde stijfheid van deze ondersteuning ondervangt de eerdergenoemde problematiek die optreedt bij het gebruik van soft mounts. Daarbij beoogt dit isolatieconcept de damping van de suspensiemodes alsmede relevante structurele resonantiemodes te verbeteren terwijl tegelijkertijd de behaalde isolatie van vloertrillingen vergelijkbaar moet zijn met die van "highend" soft mount systemen.

Teneinde de demping van de suspensiemodes en structurele modes te verbeteren wordt een feedbackregeling toegepast. Het blijkt dat hiervoor absolute bewegingssensoren (acceleratiesensoren of geofoons) beter geschikt zijn dan kracht- of verplaatsingssensoren. Daarnaast zijn voorwaarden afgeleid waarvoor het systeem (met absolute bewegingssensoren) collocated blijft. In de meeste praktische gevallen zal aan deze voorwaarden voldaan worden, waardoor een robuust stabiele feedbackregeling relatief eenvoudig te realiseren is.

Om verdere reductie van de overdracht van vloertrillingen te bewerkstelligen wordt feedforwardcompensatie van gemeten vloertrillingen toegepast. De optimale feedforwardcompensatie is gedeeltelijk afhankelijk van systeemdynamica die in het algemeen moeilijk te achterhalen is. Daarom zijn adaptieve algoritmes gebruikt om de regelaarparameters real-time aan te passen en zodoende de optimale feedforwardcompensatie te benaderen. 
Als uitgangspunt is het Filtered-reference Least Mean Squares (FxLMS) algoritme genomen, dat veel gebruikt wordt in het aanverwante veld van actieve geluidsreductie (Active Noise Control). Hierbij wordt meestal een filterparametrisatie gebruikt met een eindige impuls respons (het zogenaamde FIRfilter), omdat het gebruikte optimalisatiecriterium (de momentane gekwadrateerde fout) dan een globaal minimum heeft. Bovendien is het algoritme redelijk ongevoelig voor modelfouten. Enkele bestaande extensies van het FxLMS algoritme zijn gecombineerd om de convergentiesnelheid te verbeteren en om saturatie te voorkomen van het actuatorsignaal. Helaas vereist de FIRparametrisatie een groot aantal parameters om de optimale feedforward controller met voldoende nauwkeurigheid te benaderen. Tengevolge hiervan is de beschikbare rekencapaciteit een belangrijke beperkende factor voor te behalen prestaties, met name voor meerkanaals (MIMO) systemen.

Als een alternatief is in dit proefschrift een Infinite Impulse Response (IIR) parametrisatie met vaste polen voorgesteld. Dezelfde adaptieve algoritmes kunnen worden gebruikt voor deze parametrisatie en het optimalisatieprobleem heeft nog steeds een globaal minimum. Met deze parametrisatie kan een vergelijkbare trillingsisolatie worden bereikt met minder parameters, wat resulteert in minder stringente eisen aan de rekencapaciteit. Echter, de prestaties zijn sterk afhankelijk van de keuze van de vaste polen in het IIR-filter.

In het algemeen is een statisch bepaald mechanisch ontwerp gewenst om moeilijk voorspelbare vervormingen door bijvoorbeeld thermische belasting te voorkomen. Bovendien worden tevens de prestaties van het feedback- en feedforward-geregelde systeem negatief beinvloed wanneer afgeweken wordt van een statisch bepaald ontwerp. Op basis van de analyse van een relatief eenvoudig planair model is een richtlijn gevonden voor de verhouding tussen principale en parasitaire stijfheid van de ondersteuning: om de invloed van parasitaire stijfheden te reduceren tot een aanvaardbaar niveau moet deze stijfheidsverhouding tenminste 100 bedragen (bij gebruik van absolute bewegingssensoren).

Een generiek model voor absolute bewegingssensoren is gebruikt om eisen op te stellen voor zulke sensoren bij gebruik in hard mount trillingsisolatiesystemen. Het blijkt dat de eisen, in termen van bandbreedte en het equivalente ruisniveau, zeer stringent zijn. Er zijn geen sensoren gevonden die voldoen aan deze eisen over het gehele frequentiebereik. Als gevolg zijn de ruiseigenschappen van de sensoren een belangrijke beperkende factor in de behaalde trillingsisolatie.

De feedback- en feedforward-strategieën zijn experimenteel geverifieerd op een hard mount experimentele opstelling met één dominante bewegingsrichting. De experimenten tonen aan dat de feedbackregeling significante demping in de suspensiemode $(\zeta>70 \%)$ en de structurele mode $(\zeta>30 \%) \mathrm{kan}$ realiseren. 
De toegenomen demping vermindert het RMS versnellingsniveau met 15-20 $\mathrm{dB}$ ten opzichte van de open lus respons. Bij een RMS vloerversnelling input van ca. $20 \mathrm{~mm} / \mathrm{s}^{2}(0-1600 \mathrm{~Hz})$, is de adaptieve feedforwardcompensatie (in combinatie met de feedback-regeling) in staat om het acceleratieniveau verder terug te brengen tot $0,8-1,7 \mathrm{~mm} / \mathrm{s}^{2}$ RMS (circa -27 tot $-21 \mathrm{~dB}$ ).

De experimenteel behaalde isolatie van vloertrillingen is nog niet vergelijkbaar met de prestaties van "high-end" soft mount systemen. De resulaten zijn echter bemoedigend, zeker wanneer in acht wordt genomen dat de ondersteuning van de experimentele opstelling een factor 300 stijver is dan een "highend" soft mount. De belangrijkste beperkende factoren voor het systeem zijn het ruisniveau van de bewegingssensoren en tijdsvertraging veroorzaakt door de anti-aliasing filters.

Om de trillingsisolatie van het hard mount systeem verder te verbeteren is verder onderzoek noodzakelijk. Dit onderzoek moet zich richten op implementatie op een meerkanaals opstelling, efficiëntere implementatie van de adaptieve algoritmes en verbetering van de gebruikte sensoren. 



\section{Dankwoord}

Het heeft even geduurd, maar eindelijk ligt hier dan mijn proefschrift. Voor de totstandkoming van dit werk (en zeker voor de laatste loodjes) ben ik veel mensen dank verschuldigd.

Allereerst wil ik mijn begeleiders Herman Soemers, Ben Jonker en Johannes van Dijk bedanken voor de prettige samenwerking, en met name voor het aangeven van aandachtspunten, hun bijdrage in onze discussies en het corrigeren van (veel) teksten. In het bijzonder wil ik Johannes bedanken voor zijn dagelijkse begeleiding, waarbij zijn deur altijd open stond voor een moment van discussie of overleg.

Het IOP wil ik bedanken voor de geboden kans om dit project binnen het kader van het Precisietechnologie programma uit te voeren. Daarbij ben ik ook dank verschuldigd aan de leden van de begeleidingscommissie en mijn mede-onderzoekers binnen het IOP-project, Tom Basten en Michiel Vervoordeldonk, voor hun interesse en rol als sparringpartners.

Daarnaast wil ik Xiaohu, Gerke, Stefan, Rommert, Rob, Vincent, Ramy en Rudolf bedanken voor de bijdrage die zij met hun afstudeeropdrachten in de afgelopen jaren hebben geleverd. Ik heb hen met veel plezier begeleid. Ondanks dat niet al hun resultaten een plaats hebben gekregen in dit proefschrift, is hun werk wel de katalysator geweest voor het hier gepresenteerde werk. Bovendien wordt deze onderzoekslijn in de komende jaren voortgezet, waarbij hun werk als uitgangspunt zal dienen.

Van de vakgroep Werktuigbouwkundige Automatisering wil ik alle collega's en oud-collega's bedanken voor de prettige samenwerking gedurende de afgelopen jaren. Ik denk met name met veel plezier terug aan de koffiepauzes (met de steevaste grappen en grollen) en de (notoire) stapavonden. Speciaal wil ik Leo Tiemersma bedanken voor alle hulp met mijn experimentele opstellingen. Elke aanpassing of gewenste uitbreiding (dat waren er niet weinig) heeft hij altijd zeer voortvarend gerealiseerd. Tevens wil ik Martina Tjapkes-Hornig bedanken voor alle organisatorische hulp bij conferentiebezoeken, boekingen en het regelen van diverse zaken omtrent mijn promotie. Als laatste (maar zeker niet de minste) van de groep collega's wil ik Dirk Tjepkema bedanken voor zijn steun in het afgelopen jaar, als collega, maar belangrijker nog, als vriend. Met etentjes, borrels (“één glaasje wijn...") en sport heeft hij de broodnodige momenten van ontspanning weten in te lassen gedurende de laatste loodjes. 
Gedurende de afgelopen jaren heb ik met veel plezier gebasketbald bij D.B.V. Arriba. De combinatie van fanatiek trainen en presteren op het veld alsmede tijdens de derde helft waren een goede manier om de zorgen over en gedachten aan het promoveren kwijt te raken, ook al lukte dat niet altijd even goed. Alle teamgenoten en trainers van de afgelopen jaren: bedankt voor alles! Veel van mijn vrienden wil ik bedanken voor hun begrip dat ik het afgelopen jaar weinig van me heb laten horen en zien. Ik hoop dit in de komende periode weer goed te kunnen maken. Als allerlaatste wil ik mijn ouders, mijn broer en mijn schoonzus bedanken voor hun onvoorwaardelijke steun gedurende mijn promotie en de voorafgaande studie. Dankzij hun steun zie ik de toekomst met vertrouwen tegemoet.

Tjeerd van der Poel

Enschede, april 2010 


\section{Publications}

G.W. van der Poel, J. van Dijk, J.B. Jonker, and H.M.J.R. Soemers (2006), Control design for hard mount vibration isolation in high-precision machinery, in Int. Congr. Sound Vib. (ICSV13), ISBN 3-6501554-4-9.

G.W. van der Poel, J. van Dijk, J.B. Jonker, and H.M.J.R. Soemers (2007a), Improving the vibration isolation performance of hard mounts for precision equipment, in Intern. Conf. Adv. Intell. Mechatron. (AIM2007), IEEE/ASME, ISBN 1-4244-1264-1, CD-ROM.

G.W. van der Poel, J. van Dijk, J.B. Jonker, and H.M.J.R. Soemers (2007b), Improving the vibration isolation performance of hard mounts; a combined approach using feedback and adaptive FxLMS-based feedforward control, in Philips Conf. on Appl. Contr. Technol. (PACT).

G.W. van der Poel, J. van Dijk, J.B. Jonker, and H.M.J.R. Soemers (2008), Selection of motion sensors for hard mount active vibration isolation, in Intern. Conf. Motion Vibr. Contr. (MOVIC), CD-ROM.

G.W. van der Poel (2009), Active vibration isolation using stiff supports, in Philips Conf. on Appl. Contr. Technol. (PACT), pages 57-60. 



\section{Bibliography}

C. Acar and A.M. Shkel (2003), Experimental evaluation and comparative analysis of commercial variable-capacitance MEMS accelerometers, J. Micromech. Microeng., 13:634-645.

H.J.M.T.A. Adriaens, W.L. de Koning, and R. Banning (2000), Modeling piezoelectric actuators, IEEE/ASME Trans. Mechatron., 5(4):331-341.

H. Amick, S. Hardash, P. Gillett, and R.J. Reaveley (1991), Design of stiff, lowvibration floor structures, in Proc. SPIE: Vib. Contr. Microelectron. Opt. Metrol., vol. 1619, pages 180-191.

P.E. An, M. Brown, and C.J. Harris (1997), On the convergence rate performance of the normalized least-mean-square adaptation, IEEE Trans. Neural Networks, 8(5):1211-1214.

Analog Devices (2002), OP37 Datasheet Rev. B, Technical report, Analog Devices, available from http://www.analog.com, last accessed July 2009.

Analog Devices (2006), OP27 Datasheet Rev. F, Technical report, Analog Devices, available from http://www.analog.com, last accessed July 2009.

E.H. Anderson and J.P. How (1997), Active vibration isolation using adaptive feedforward control, in Proc. Am. Contr. Conf. (ACC), pages 1783-1788.

K.J. Åström, P. Hagander, and J. Sternby (1984), Zeros of sampled systems, Automatica, 20(1):31-38.

M.J. Balas (1979), Direct velocity feedback control of large space structures, J. Guid. Contr., 2(3):252-253.

R. Banning, W.L. de Koning, H.J.M.T.A. Adriaens, and R.K. Koops (2001), State-space analysis and identification for a class of hysteretic systems, Automatica, 37:1883-1892.

J. Bao and P.L. Lee (2007), Process control: The passive systems approach, Springer, ISBN 978-1-84628-892-0. 
A. Barzilai, T. VanZandt, and T. Kenny (1998), Technique for measurement of the noise of a sensor in the presence of large background signals, Rev. Sci. Instr., 69(7):2767-2772.

A.M. Beard, D.W. Schubert, and A.H. von Flotow (1994), A practical product implementation of an active/passive vibration isolation system, in Proc. SPIE: Vib. Monit. Contr., vol. 2264, pages 38-49.

C.A.J. Beijers (2005), A modeling approach to hybrid isolation of structureborne sound, PhD thesis, University of Twente, ISBN 90-365-2189-0.

R. Ben Mrad and H. Hu (2002), A model for voltage-to-displacement dynamics in piezoceramic actuators subject to dynamic-voltage excitations, IEEE/ASME Trans. Mechatron., 7(4):479-489.

A.P. Berkhoff (2006), A modified filtered-error algorithm with fast convergence in systems with delay, in Proc. Int. Symp. Active Control Sound Vib. (ACTIVE).

A.P. Berkhoff and G. Nijsse (2007), A rapidly converging filtered-error algorithm for multichannel active noise control, Int. J. Adapt. Control Signal Process., 21(7):556-569.

W. Bialowons, R. Amirikas, A. Bertolini, and D. Krücker (2007), Measurement of ground motion in various sites, Technical report 2007-011, EUROTeV, available from http://www.eurotev.org, last accessed December 2008.

E. Bjarnason (1992), Active noise cancellation using a modified form of the Filtered-X LMS algorithm, in Proc. European Signal Process. Conf. (EUSIPCO), vol. II, pages 1053-1056.

D.L. Blanding (1999), Exact constraint: Machine design using kinematic processing, ASME Press, ISBN 978-0-7918-0085-0.

C.I. Byrnes, A. Isidori, and J.C. Willems (1991), Passivity, feedback equivalence, and the global stabilization of minimum phase nonlinear systems, IEEE Trans. Autom. Contr., 36(11):1228-1240.

B.M. Chen, Z. Lin, and Y. Shamash (2004), Linear systems theory: A structural decomposition approach, Birkhäuser, ISBN 978-0-8176-3779-8.

F. Claeyssen, R. Le Letty, F. Barillot, and O. Sosnicki (2007), Amplified piezoelectric actuators: Static \& dynamic applications, Ferroelectrics, 351(1): $3-14$.

R.G. Cobb, J.M. Sullivan, A. Das, L. Porter Davis, T. Tupper Hyde, T. Davis, Z.H. Rahman, and J.T. Spanos (1999), Vibration isolation and suppression system for precision payloads in space, Smart Mater. Struct., 8:798-812. 
M.J. Crosby and D.C. Karnopp (1973), The active damper: A new concept for shock and vibration control, Shock Vib. Bull., 43:119-133.

B. de Moor (1993), The singular value decomposition and long and short spaces of noisy matrices, IEEE Trans. Signal Process., 41(9):2826-2838.

S.C. Douglas (1997), Fast exact Filtered-X LMS and LMS algorithms for multichannel active noise control, in IEEE Int. Conf. Acoust. Speech Signal Process., vol. 1, pages 399-402.

S.C. Douglas (1999), Fast implementations of the Filtered-X LMS and LMS algorithms for multichannel active noise control, IEEE Trans. Speech Audio Process., 7(4):454-465.

S.J. Elliott (1998), Filtered reference and filtered error LMS algorithms for adaptive feedforward control, Mechanical Syst. Signal Process., 12(6):769781.

S.J. Elliott (2000), Optimal controllers and adaptive controllers for multichannel feedforward control of stochastic disturbances, IEEE Trans. Signal Process., 48(4):1053-1060.

S.J. Elliott (2001), Signal processing for active control, Academic Press, ISBN 978-0-12-237085-4.

S.J. Elliott and J.G. Cook (2000), A preconditioned LMS algorithm for rapid adaptation of feedforward controllers, in Proc. IEEE Int. Conf. Acoust. Speech Signal Process. (ICASSP), vol. 2, pages II845-II848.

EuroTeV (WWW), European design study towards a global TeV linear collider, http://www.eurotev.org.

K. Faber and P.W. Maxwell (1997), Geophone spurious frequency: What is it and how does it affect seismic data quality?, Can. J. Explor. Geophys., 33 (1\&2):46-54.

H. Fan, Y. Yang, and M. Nayeri (1988), On the performance surfaces of a frequency domain adaptive IIR filter, in Proc. Int. Conf. Acoust. Speech Signal Process., vol. 3, pages 1564-1567.

A. J. Fleming and S.O.R. Moheimani (2004), Improved current and charge amplifiers for driving piezoelectric loads, and issues in signal processing design for synthesis of shunt damping circuits, J. Intell. Mater. Syst. Struct., 15:77-92.

P.R. Fraanje (2004), Robust and fast schemes in broadband active noise and vibration control, PhD thesis, University of Twente, ISBN 978-90-901800$2-1$. 
R. Fraanje, M. Verhaegen, and N. Doelman (2004), Increasing the robustness of a preconditioned Filtered-X LMS algorithm, IEEE Signal Process. Letters, 11(2):285-288.

R. Fraanje, S.J. Elliott, and M. Verhaegen (2007), Robustness of the Filtered-X LMS algorithm - part II: Robustness enhancement by minimal regularization for norm bounded uncertainty, IEEE Trans. Signal Process., 55(8): 4038-4047.

G.F. Franklin, J.D. Powell, and A. Emami-Naeini (1994), Feedback control of dynamic systems, Addison-Wesley, $3^{\text {rd }}$ edition, ISBN 978-0-201-53487-0.

A. Friedrich, F. Krüger, and K. Klinge (1998), Ocean-generated microseismic noise located with the Gräfenberg array, J. Seismolog., 2:47-64.

C.R. Fuller, S.J. Elliott, and P.A. Nelson (1997), Active control of vibration, Academic Press, $2^{\text {nd }}$ edition, ISBN 978-0-12-269441-7.

T.B. Gabrielson (1993), Mechanical-thermal noise in micromachined acoustic and vibration sensors, IEEE Trans. Electron Devices, 40(5):903-909.

T.B. Gabrielson (1995), Fundamental noise limits for miniature acoustic and vibration sensors, J. Vib. Acoust., 117:405-410.

P. Ge and M. Jouaneh (1995), Modeling hysteresis in piezoceramic actuators, Precis. Eng., 17(3):211-221.

M. Gendreau (1999), Effects of acoustic noise on optical tools, in Proc. SPIE: Optomechanical Engineering and Vibration Control, vol. 3786, pages 4-13.

Z.J. Geng and L.S. Haynes (1994), Six degree-of-freedom active vibration control using the Stewart platforms, IEEE Trans. Contr. Syst. Technol., 2(1): 45-53.

M. Goldfarb and N. Celanovic (1996), Behavioral implications of piezoelectric stack actuators for control of micromanipulation, in Proc. IEEE Int. Conf. Robotics Autom., vol. 1, pages 226-231.

M. Goldfarb and N. Celanovic (1997), Modeling piezoelectric stack actuators for control of micromanipulation, IEEE Contr. Syst., 17(3):69-79.

G.H. Golub, S. Nash, and C. van Loan (1979), A Hessenberg-Schur method for the problem $\boldsymbol{A X}+\boldsymbol{X} \boldsymbol{B}=\boldsymbol{C}$, IEEE Trans. Autom. Contr., 24(6):909-913.

C.G. Gordon (1991), Generic criteria for vibration-sensitive equipment, in Proc. SPIE: Vib. Contr. Microelectron. Opt. Metrol., vol. 1619, pages 71-85.

G. Gu (2005), All optimal Hankel-norm approximations and their $\mathcal{L}_{\infty}$ error bounds in discrete-time, Int. J. Contr., 78(6):408-423. 
Halcyonics (WWW), Nano/Micro/Vario Series, http://www.halcyonics.de, last accessed February 2010.

L.C. Hale (1999), Principles and techniques for designing precision machines, PhD thesis, University of California Livermore.

C.H. Hansen and S.D. Snyder (1997), Active control of noise and vibration, Chapman \& Hall, ISBN 978-0-419-19390-6.

C.M. Harris and A.G. Piersol (2002), Harris' Shock and vibration handbook, McGraw-Hill, $5^{\text {th }}$ edition, ISBN 978-0-07-137081-3.

G.S. Hauge and M.E. Campbell (2004), Sensors and control of a space-based six-axis vibration isolation system, J. Sound Vib., 269:913-931.

S. Haykin (2002), Adaptive filter theory, Prentice-Hall, $4^{\text {th }}$ edition, ISBN 978-0-13-090126-2.

M. Heertjes, K. de Graaff, and J.-G. van der Toorn (2005), Active vibration isolation of metrology frames: A modal decoupled control design, J. Vib. Acoust., 127:223-233.

P.S.C. Heuberger, P.M.J. van den Hof, and O.H. Bosgra (1993), A generalized orthonormal basis for linear dynamical systems, in Proc. $32^{\text {nd }}$ Conf. Decision Control (CDC), vol. 3, pages 2850-2855.

P.S.C. Heuberger, P.M.J. van den Hof, and B. Wahlberg (eds.) (2005), Modelling and identification with rational orthogonal basis functions, SpringerVerlag, ISBN 1-85233-956-X.

C.-W. Ho, A.E. Ruehli, and P.A. Brennan (1975), The modified nodal approach to network analysis, IEEE Trans. Circuits Syst., 22:504-509.

L.G. Holcomb (1989), A direct method for calculating instrument noise levels in side-by-side seismometer evaluations, Technical report OFR 89-214, U.S. Geol. Surv., available from http://www.usgs.gov, last accessed July 2009.

J. Holterman (2002), Vibration control of high-precision machines with active structural elements, PhD thesis, University of Twente, ISBN 978-90-3651793-5.

J. Holterman, T.J.A. de Vries, and F. Auer (2002), Active damping based on decoupled collocated control, in Proc. Int. Symp. Active Contr. Sound Vib. (ACTIVE), pages 827-838.

C.Q. Howard and C.H. Hansen (2003), Vibration analysis of waffle floors, Comput. Struct., 81:15-26.

T.T. Hyde and E.F. Crawley (1995), $\mathcal{H}_{2}$ synthesis for active vibration isolation, in Proc. Am. Contr. Conf. (ACC), pages 3835-3839. 
V. Ionescu and C. Oară (1996), Spectral and inner-outer factorizations for discrete-time systems, IEEE Trans. Autom. Contr., 41(12):1840-1845.

L. Jabben (2007), Mechatronic design of a magnetically suspended rotating platform, PhD thesis, Delft University of Technology, The Netherlands, ISBN 978-90-9022523-4.

L. Jabben, D.L. Trumper, and J. van Eijk (2008), Dynamic Error Budgeting an integral system design approach for high precision machines, in Proc. euspen Int. Conf., vol. I, pages 363-367.

J.B. Jonker, R.G.K.M. Aarts, and J. van Dijk (2009), A linearized input-output representation of flexible multibody systems for control synthesis, Multibody Syst. Dyn., 21:99-122.

S.M. Joshi (1985), Robustness of velocity feedback controllers for flexible spacecraft, IEEE Trans. Aerosp. Electron. Syst., 21(1):2-7.

M.P. Koster (1998), Constructieprincipes voor het nauwkeurig bewegen en positioneren, Twente University Press, ISBN 978-90-365-1135-3, in Dutch.

S.M. Kuo and D.R. Morgan (1996), Active noise control systems: Algorithms and DSP implementations, John Wiley \& Sons, ISBN 978-0-471-13424-4.

S.M. Kuo and D.R. Morgan (1999), Active noise control: A tutorial review, Proc. IEEE, 87(6):943-973.

S.M. Kuo and Y. Yang (1996), Broadband adaptive noise equalizer, IEEE Signal Process. Letters, 3(8):234-235.

I.D. Landau, R. Lozano, and M. M'Saad (1998), Adaptive control, Springer, ISBN 978-3-540-76187-7.

F.A. Levinzon (2004), Fundamental noise limit of piezoelectric accelerometer, IEEE Sens. J., 4(1):108-111.

F.A. Levinzon (2005), Noise of piezoelectric accelerometer with integral FET amplifier, IEEE Sens. J., 5(6):1235-1242.

F.H. Ling, W.M. Wang, and R.J. Mao (1990), Dynamic stability analysis of the geophone spurious frequency phenomenon, J. Sound Vib., 139(1):21-30.

L. Ljung (1999), System identification: Theory for the user, Prentice-Hall, $2^{\text {nd }}$ edition, ISBN 978-0-13-656695-3.

P.A.C. Lopes and M.S. Piedade (2004), The behavior of the Modified FX-LMS algorithm with secondary path modeling errors, IEEE Signal Process. Letters, 11(2):148-151. 
T. McKelvey, H. Akçay, and L. Ljung (1996), Subspace-based multivariable system identification from frequency response data, IEEE Trans. Autom. Contr., 41(7):960-979.

Minus K Technology (WWW), http://www.minusk.com, last accessed February 2010.

G.E. Moore (1965), Cramming more components onto integrated circuits, Electron., 38(8):114-117.

Y. Nakamura, M. Nakayama, K. Masuda, K. Tanaka, M. Yasuda, and T. Fujita (2000), Development of active six degrees-of-freedom microvibration control system using giant magnetostrictive actuators, Smart Mater. Struct., 9 (2):175-185.

H. Nakazawa (1994), Principles of precision engineering, Oxford University Press, ISBN 978-0-19-856266-5.

V.H. Nascimento and A.H. Sayed (1996), An unbiased and cost-effective leakyLMS filter, in Proc. $30^{\text {th }}$ Asilomar Conf. Signals Syst. Computers, vol. 2, pages $1078-1082$.

V.H. Nascimento and A.H. Sayed (1999), Unbiased and stable leakage-based adaptive filters, IEEE Trans. Signal Process., 47(12):3261-3276.

M. Nayeri and W.K. Jenkins (1989), Alternate realizations to adaptive IIR filters and properties of their performance surfaces, IEEE Trans. Circuits Syst., 36(4):485-496.

P.G. Nelson (1998), Suspended low-frequency horizontal pendulum isolator for vibration isolation systems, US Patent 5,779,010.

G. Nijsse (2006), A subspace based approach to the design, implementation and validation of algorithms for active vibration isolation control, $\mathrm{PhD}$ thesis, University of Twente, ISBN 978-90-365-1916-8.

Noliac website (WWW), PZT material data, http://www.noliac.com, last accessed July 2009.

H. Nyquist (1928), Thermal agitation of electric charge transducers, Phys. Rev., 32(1):110-113.

T. Oliveira e Silva (1995), On the determination of the optimal pole position of Laguerre filters, IEEE Trans. Signal Process., 43(9):2079-2087.

R. Ortega, A. Loría, P.J. Nicklasson, and H. Sira-Ramírez (1998), Passivitybased control of Euler-Lagrange systems, Springer, ISBN 978-1-85233016-3. 
A. Partridge, J.K. Reynolds, B.W. Chui, E.M. Chow, A.M. Fitzgerald, L. Zhang, N.I. Maluf, and T.W. Kenny (2000), A high-performance planar piezoresistive accelerometer, J. Microelectromech. Syst., 9(1):58-66.

J. Peterson (1993), Observations and modeling of seismic background noise, Technical report OFR 93-322, U.S. Geol. Surv., available from http://www.usgs.gov, last accessed March 2009.

M. Petyt and W.H. Mirza (1972), Vibration of column-supported floor slabs, J. Sound Vib., 21(3):355-364.

R. Pintelon and J. Schoukens (2001), System identification: A frequency domain approach, IEEE Press, ISBN 978-0-7803-6000-6.

D.L. Platus (1999), Negative-stiffness-mechanism vibration isolation systems, in Proc. SPIE: Optomech. Eng. Vib. Contr., vol. 3786, pages 98-105.

A. Preumont (2002), Vibration control of active structures: An introduction, vol. 96 of Solid Mechanics and its Applications, Kluwer Academic, $2^{\text {nd }}$ edition, ISBN 978-1-4020-0496-4.

A. Preumont, R. François, F. Bossens, and A. Abu Hanieh (2002), Force feedback versus acceleration feedback in active vibration isolation, J. Sound Vib., 257(4):605-613.

S. Redaelli, R.W. Aßmann, W. Coosemans, and W. Schnell (2002), The effect of cooling water on magnet vibrations, in Proc. Eur. Part. Accel. Conf. (EPAC), pages 485-487.

S. Redaelli, R.W. Aßmann, W. Coosemans, G. Guignard, D. Schulte, I. Wilson, and F. Zimmermann (2004), CLIC magnet stabilization studies, in Proc. Int. Lin. Accel. Conf. (LINAC), Technical report CERN-AB-2004-063, CERN, available from http://cdsweb.cern.ch, last accessed February 2009.

P.A. Regalia (1992), Stable and efficient lattice algorithms for adaptive IIR filtering, IEEE Trans. Signal Process., 40(2):375-388.

P.A. Regalia (1995), Adaptive IIR filtering in signal processing and control, Marcel Dekker, ISBN 0-8247-9289-0.

M.A. Riedesel, R.D. Moore, and J.A. Orcutt (1990), Limits of sensitivity of inertial seismometers with velocity transducers and electronic amplifiers, Bull. Seismol. Soc. Am., 80(6):1725-1752.

N.B. Roozen and M.J. Vervoordeldonk (2007), Prediction and control of acoustically induced vibrations of high-precision equipment, in Proc. ASPE Annu. Meet. 
N.B. Roozen, B.T. Verhaar, and M.J. Vervoordeldonk (2006), Acoustic excitation of mechatronic systems by diffuse acoustic sound fields: Numerical predictions and measurements, in Proc. Int. Conf. Noise Vib. Eng. (ISMA), (CD-ROM).

D.J. Rossetti, M.R. Jolly, and S.C. Southward (1996), Control effort weighting in feedforward adaptive control systems, J. Acoust. Soc. Am., 99(5):29552964.

M. Rupp and A.H. Sayed (1996), A time-domain feedback analysis of filterederror adaptive gradient algorithms, IEEE Trans. Signal Process., 44(6): 1428-1439.

M. Rupp and A.H. Sayed (1998), Robust FxLMS algorithms with improved convergence performance, IEEE Trans. Speech Audio Process., 6(1):78-85.

M.G. Safonov and R.Y. Chiang (1989), A Schur method for balancedtruncation model reduction, IEEE Trans. Autom. Contr., 34(7):729-733.

M.G. Safonov, R.Y. Chiang, and D.J.N. Limebeer (1990), Optimal Hankel model reduction for nonminimal systems, IEEE Trans. Autom. Contr., 35 (4):496-502.

P.M. Sain, M.K. Sain, and B.F. Spencer (1997), Models for hysteresis and application to structural control, in Proc. Am. Contr. Conf. (ACC), pages 1620.

A.H. Sayed (2003), Fundamentals of adaptive filtering, John Wiley \& Sons, ISBN 978-0-471-46126-5.

A.H. Sayed and Th. Kailath (2001), A survey of spectral factorization methods, Numer. Linear Algebr., 8:467-496.

B.K.P. Scaife (1998), Principles of dielectrics, Oxford University Press, ISBN 978-0-19-856557-4.

P. Schellekens, N. Rosielle, H. Vermeulen, M. Vermeulen, S. Wetzels, and W. Pril (1998), Design for precision: Current status and trends, in Annals of CIRP, vol. 47(2), pages 557-586.

G. Schitter, R.W. Stark, and A. Stemmer (2002), Sensors for closed-loop piezo control: Strain gauges versus optical sensors, Meas. Sci. Technol., 13:N47N48.

D.W. Schubert, A.M. Beard, S.F. Shedd, M.R. Earles, Jr, and A.H. von Flotow (1998), Stiff actuator active vibration isolation system, US Patent 5,823,307.

A. Sery and O. Napoly (1996), Influence of ground motion on the time evolution of beams in linear colliders, Phys. Rev. E, 53(5):5323-5337. 
J.J. Shynk (1989)a, Adaptive IIR filtering, IEEE ASSP Mag., 6(2):4-21.

J.J. Shynk (1989)b, Adaptive IIR filtering using parallel-form realizations, IEEE Trans. Acoust. Speech Signal Process., 37(4):519-533.

S. Skogestad and I. Postlethwaite (2005), Multivariable feedback control: Analysis and design, John Wiley \& Sons, $2^{\text {nd }}$ edition, ISBN 978-0-470-01167-6.

SLAC (WWW), International Linear Collider (ILC) at SLAC National Accelerator Laboratory, http://www-project.slac.stanford.edu/ilcm.

A.H. Slocum (1992), Precision machine design, Prentice-Hall, ISBN 978-087263-492-3.

J. Spanos, Z. Rahman, and G. Blackwood (1995), A soft 6-axis active vibration isolator, in Proc. Am. Contr. Conf. (ACC), pages 412-416.

Technical Manufacturing Corporation (TMC) (WWW), STACIS ${ }^{\circledR}$ active piezoelectric vibration cancellation system, http://www.techmfg.com, last accessed February 2010.

Texas Instruments (2007), Noise analysis in operational amplifier circuits, Technical report SLVA043 Rev. B, Texas Instruments, available from http://www.ti.com, last accessed July 2009.

A. Tzes and K. Le (1996), Application of frequency domain adaptive infinite impulse response filtering for identification of flexible structure dynamics, Mechanical Syst. Signal Process., 10(1):65-91.

P.M.J. van den Hof, P.S.C. Heuberger, and J. Bokor (1995), System identification with generalized orthonormal basis functions, Automatica, 31(12): $1821-1834$.

A. van der Schaft (2000), $\mathcal{L}_{2}$-gain and passivity techniques in nonlinear control, Springer-Verlag, ISBN 978-1-85233-073-6.

B.J.G. Vautier and S.O.R. Moheimani (2005), Charge driven piezoelectric actuators for structural vibration control: Issues and implementation, Smart Mater. Struct., 14:575-586.

P. Verboven, P. Guillaume, S. Vanlanduit, and B. Cauberghe (2006), Assessment of nonlinear distortions in modal testing and analysis of vibrating automotive structures, Sound Vib., 293:299-319.

M.J. Vervoordeldonk, T.A.M. Ruijl, and R.M.G. Rijs (2004), Development of a novel active isolation concept, in Proc. ASPE Top. Meet.: Contr. Precis. Syst.

M.J. Vervoordeldonk, J. van Eijk, and N.B. Roozen (2006), Active structural damping in high-precision equipment, in Proc. Int. Conf. Noise Vib. Eng. (ISMA), pages 573-581. 
M.J. Vervoordeldonk, T.A.M. Ruijl, R.M.G. Rijs, and J.C.A. Muller (2007), Actuator arrangement for active vibration isolation comprising an inertial reference mass, US Patent Application 2007/0035074 A1.

M. Vidyasagar (1985), Control systems synthesis: A factorization approach, MIT Press, Cambridge, MA, USA.

A. Visscher (2006), Personal communication, FEI Company.

B. Wahlberg (1994), System identification using Kautz models, IEEE Trans. Autom. Contr., 39(6):1276-1282.

E.A. Wan (1996), Adjoint LMS: An efficient alternative to the Filtered-X LMS and multiple error LMS algorithms, in Proc. IEEE Int. Conf. Acoust. Speech Signal Process. (ICASSP), vol. 3, pages 1842-1845.

A.K. Wang and W. Ren (1999), Convergence analysis of the multi-variable Filtered-X LMS algorithm with application to active noise control, IEEE Trans. Signal Process., 47(4):1166-1169.

L.M. Wedepohl and L. Jackson (2002), Modified nodal analysis: An essential addition to electrical circuit theory and analysis, Eng. Sci. Educ. J., 11(3): 84-92.

P.D. Welch (1967), The use of Fast Fourier Transform for the estimation of power spectra: A method based on time averaging over short, modified periodograms, IEEE Trans. Audio Electroacoust., AU-15(2):70-73.

J.M. Wesselink (2009), A rapid prototyping system for broadband multichannel active noise and vibration control, PhD thesis, University of Twente, ISBN 978-90-365-2936-5.

B. Widrow and S.D. Stearns (1985), Adaptive signal processing, Prentice-Hall, ISBN 978-0-13-004029-9.

J. Winterflood, D.G. Blair, and B. Slagmolen (2002), High performance vibration isolation using springs in Euler column buckling mode, Phys. Lett. A, 300:122-130.

J. Yuan (2007), Adaptive Laguerre filters for active noise control, Applied Acoust., 68:86-96.

Y.I. Yurish (1993), Shear piezoelectric vibration pickups, Meas. Techniques, 36(12):1363-1369.

G. Zames (1981), Feedback and optimal sensitivity: Model reference transformations, multiplicative seminorms, and approximate inverses, IEEE Trans. Autom. Contr., 26(2):301-320. 
L. Zuo and S.A. Nayfeh (2004), An integral sliding control for robust vibration isolation and its implementation, in Proc. SPIE: Smart Struct. Mater.: Damping Isol., vol. 5386, pages 1-10.

L. Zuo and J.-J.E. Slotine (2005), Robust vibration isolation via frequencyshaped sliding control and modal decomposition, J. Sound Vib., 285:11231149 .

L. Zuo, J.-J.E. Slotine, and S.A. Nayfeh (2004), Experimental study of a novel adaptive controller for active vibration isolation, in Proc. Am. Contr. Conf. (ACC), vol. 4, pages 3863-3868.

L. Zuo, J.-J.E. Slotine, and S.A. Nayfeh (2005), Model reaching adaptive control for vibration isolation, IEEE Trans. Contr. Syst. Technol., 13(4):611617. 